Catalog Information on the

Performance of Aluminum in

Sea Waier

R. H. Wagner

R. A. Bonewitz

Aluminum Company of America

Alcoa Laboratories

1978 April 05

PNL -2606

UC -64

Alcoa Report

No. NXS - 3

$18 F$
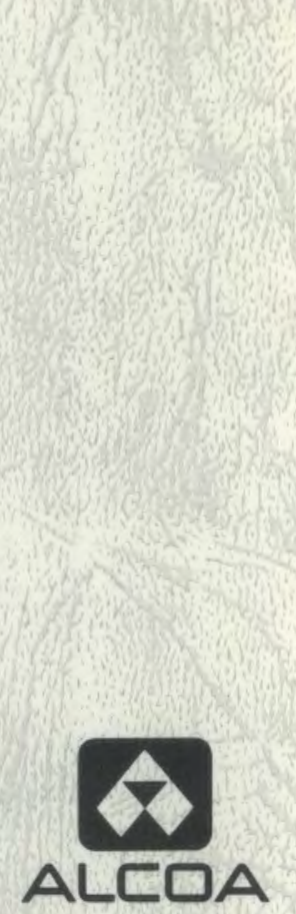

Prepared for

Pacific Northwest Laboratory

Under Agreement No. B - 31942 - A - E

Pacific Northwest Laboratory

Richland, Washington 99352

Operated for

U. S. Department of Energy

By

Battelle Memorial Institute 
PNL -2606

\title{
CATALOG INEORMATION ON THE PERFORMANCE OF ALUMINUM IN SEA WATER
}

\author{
By \\ R. H. Wagner \\ and \\ R. A. Bonewitz
}

\begin{abstract}
ALUMINUM COMPANY OF AMERICA
Alcoa Laboratories

Alcoa Technical Center Alloy Technology Division Alcoa Center, PA 15069
\end{abstract}

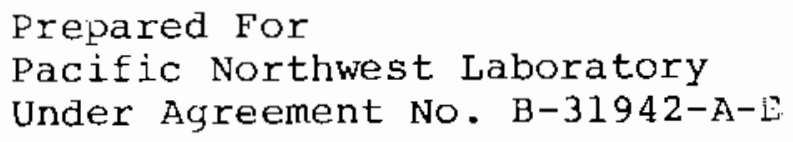

Opinions, findings, conclusions, or recomendations expressed herein are those of the authors and do not necessarily reflect the views of either DOE or FNL. 


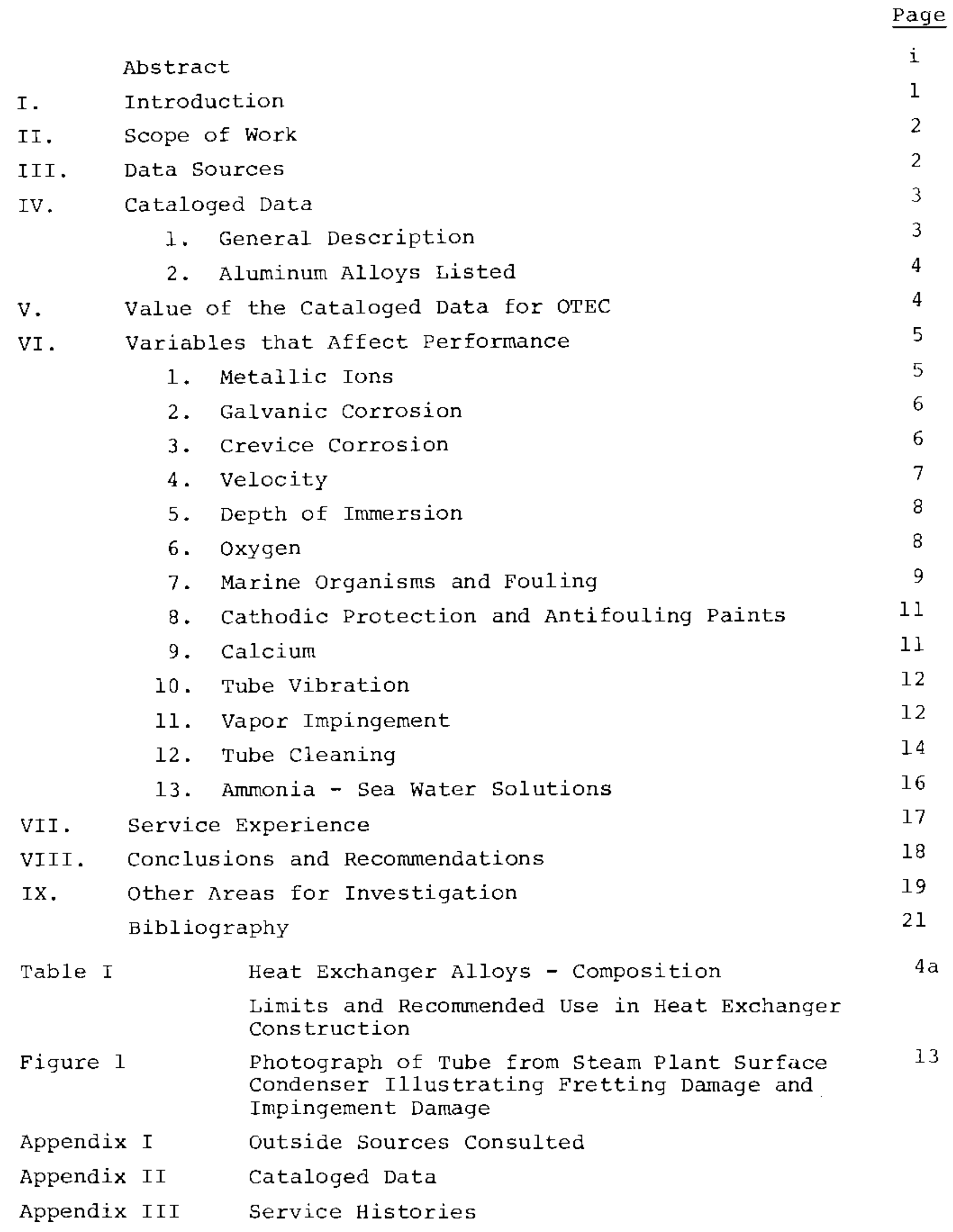


$\cdots$ 


\section{ABSTRACT}

To help evaluate aluminum alloys for OTEC heat exchanger tubes data on the performance of aluminum in surface and deep sea water has been assembled and computer cataloged. Pitting and crevice corrosion proved to be the types of attack that predominated. The cataloged data are the results of many static tests conducted under natural conditions of marine fouling, hence, they must be used carefully in qualifying aluminum for OTEC purposes. These data can serve the OTEC program best as a basis for selecting aluminum alloys that appear promising as candidates for further evaluation. The aluminum alloys 5052 and Alclad (7072) 3003 fall into this category. Available service histories also proved inadequate for qualifying aluminum alloys for the OTEC application, but they do support the selection of Alclad (7072) 3003 as a tubing alloy worthy of further consideration. Performance data covering areas of investigation peculiar to OTEC power plants is needed to permit a firm decision for or against aluminum alloy tubes. 
m 


\section{INTRODUCTION}

The Ocean Thermal Energy Conversion (OTEC) program sponsored by the United States Department of Energy, formerly the Energy Research and Development Administration (DOE/ERDA) requires the development of heat exchangers to utilize the overall temperature difference between warm water from the ocean surface and cold water from the ocean depths to set a working fluid in motion and drive a turbine. Due to the small temperature difference within the cycle these heat exchangers will have to be larger than anything in use today. The size of the units along with the fact that they will be used at sea, either floated on the surface or bouyed at some depth, makes inspection and maintenance difficult placing a premium on the corrosion resistance of construction materials.

Aluminum has recently become a candidate material for OTEC heat exchanger tubes because of its apparent compatibility with anhydrous ammonia, the proposed working fluid, its ready availability and low cost. To qualify for the OTEC application the aluminum or aluminum alloy must have a high resistance to corrosion by sea water as well as the ability to retain that resistance over an extended period of time.

Over the years aluminum alloys have enjoyed considerable acceptance for marine applications including but not limited to ship hulis, super structures, and shipboard heat exchangers $(9,47,55,59,60$, 61, 62). More recently, aluminum alloy tube, pipe, sheet, and plate have demonstrated acceptable performance handling raw and treated sea water in experimental desalination plants $(18,20,27,31,32$, $40,41,42,63)$, as well as the demonstration plants operated by Israel Desalination Ltd. (77). This does not mean that aluminum and its alloys are immune to corrosion by sea water, it means that engineering properties including corrosion resistance generally balance out in favor of aluminum for these "top side" applications.

Aluminum alloy components have performed well on the "Aluminaut" (47) and other deep submergence vehicles (54). However, in these cases the aluminum is generally protected by either special paint coatings or sacrificial anodes which can be repaired or replaced during inspection and maintenance periods. Not much can be done in the way of maintaining tubes in OTEC heat exchangers. Treating sea water with corrosion inhibitors, for example, would be financially disastrous.

To obtain information that might be of help in qualifying aluminum and/or aluminum alloys for OTEC heat exchanger tubes Alcoa entered into a contract with Battelle's Pacific Northwest Laboratories (PNL) to conduct a search of available literature on the corrosion of aluminum by sea water. 


\section{SCOPE OF WORK}

The scope of this work is briefly described in the four subdivisions that follow:

2.1 Compile and catalog data on the performance of aluminum in sea water.

2.2 Evaluate the cataloged data with respect to qualifying aluminum alloys for OTEC heat exchangers.

2.3 Provide service histories illustrating the performance of aluminum alloy tubes in sea water service.

2.4 Suggest areas where additional data is needed to qualify aluminum alloys for OTEC heat exchangers.

III. DATA SOURCES

The considerable data accumulated by the Alloy Technology Division of the Alcoa Technical Center as the result of exposure tests of aluminum in marine environments was reviewed and that pertaining to actual sea water exposures was tabulated.

In searching the literature, data bases provided by the Lockheed Information service were consulted. These included the following:

(1) Chemical Abstracts - 1972 to september, 1976.

(2) Dissertations Abstracts - All through 1976.

(3) Engineering Abstracts - 1970 to August, 1976.

(4) National Information Service - 1964 to August, 1976.

(5) Oceanic Abstracts - 1964 to August, 1976.

(6) World Aluninum Abstracts - 1968 to August, 1976.

Literature sources not covered by Lockheed that were consulted include:

(1) Corrosion Abstracts - 1962 - 1975.

(2) Chemical Abstracts - 1927 - 1972.

(3) Metal Abstracts - 1944 - 1966. 
In addition, the government laboratories, universities and other aluminum producers 1 isted in Appendix I were requested to provide pertinent data if possible. The majority of these organizations responded to the request but provided only previously published data that had been found in the literature search.

IV. CATALOGED DATA

Data on the resistance of aluminum to corrosion by sea water cataloged for the OTEC program is given in Tables $I$ and II of Appendix II. These data were cataloged using Data Processing Language and are listed according to alloy and exposure site. Al1 of the cataloged data resulted from static tests under the conditions of marine macro and/or micro fouling characteristic of the exposure site. Two tables were required because of the large amount of information needed to describe the specimen, location of exposure, sea water composition and the various measurements related to corrosion performance.

\subsection{Genera1 Description}

Table I lists the information most often provided by the references. The first five columns describe the specimen giving the alloy and temper, the product evaluated, the type of specimen evaluated, and the specimen size. The colum headed Specimen Code (Spec. Code) contains the numbers 1,2 , and 3. These code numbers are not directly related to corrosion, but have been included to indicate whether a specimen was welded or not. The code number 1 means that the specimen was not welded, a number 2 means that two pieces of the same aluminum alloy were welded together, and a number 3 means that an aluminum alloy was welded to a different aluminum alloy. Columns 7 through 9 give details of the exposure including the location of the test site, the depth of immersion in sea water and the period of time the specimens were in test. Surface water exposures are indicated by "total immersion", "mean tide" or "tide water" and "alternate immersion". The remaining columns give the corrosion information. Here one data line was sufficient for a specimen that was not welded; for a welded specimen several lines were required to record corrosion of the base alloy remote from the weld, corrosion of the base alloy in the heat affected zone adjacent to the weld, and corrosion of the weld bead. For each location examined the type, maximum and average depths of attack are given while the total volume of metal loss is recorded as a uniform corrosion rate in inches per year.

Table II provides additional sample description, miscellaneous corrosion information and a reference to the source of the data. 


\subsection{Aluminum Alloys Listed}

The cataloged data provides information on sea water corrosion for a variety of aluminum alloys including those normally recommended for heat exchanger applications. The heat exchanger alloys are designated as 3003, 5052, 5056,5083, 5456, 6061, Alclad 3003, and Alclad 6061. The composition limits and the recommended use of these alloys in heat exchanger construction are given in Table $I$.

V. VALUE OF THE CATALOGED DATA FOR OTEC

Care must be taken in utilizing the cataloged data directly for qualifying aluminum alloys for OTEC heat exchangers. This judgment is based on the following considerations:

(a) A practical interpretation of the data cannot be made because of the high degree of variability found in the depth of corrosive attack measured on specimens of any one alloy at a given exposure site.

(b) The data were obtained from static tests and do not reflect the effect of velocity which is important for OTEC.

(c) The data were obtained under the conditions of marine macro and/or micro fouling characteristic of the site and depths, therefore, they do not show the effect of clean surfaces as required for OTEC heat exchangers.

The cataloged data can serve the OTEC program best as a basis for selecting aluminum alloys for additional evaluation. These data indicate that the nonclad aluminum alloy 5052 and the clad alloy Alclad 3003 are good candidates for testing under conditions that approximate those anticipated for OTEC. That these two alloys merit further consideration in the OTEC program is best illustrated by data reported by Jenkins and Reinhart (2) and by Reinhart (3) for exposures in surface and deep water of the Pacific Ocean off Port Hueneme, California. Their data for the 5052 aluminum alloy is given on pages $9,11,12$, and 13 in Table $I$ and pages 8,19 , 11, and 12 in Table II of the cataloged data. The data in Table I shows that the 5052 alloy has a high resistance to pitting corrosion that was not significantly affected by either length of exposure or depth of immersion. The data in Table II confirms that the 5052 alloy like most aluminum and other conmon metal alloys is susceptible to crevice corrosion. A critical evaluation of crevice corrosion, with respect to alloy, length of exposure and depth of immersion is impossible without assurance that all of the crevice specimens were uniform in nature. In the opinion of the authors, resistance 
Table I

HEAT EXCHANGER ALLOYS - THEIE COMPOSITION LIMITE AND RECOMMENDED USE IN HEAT EXCHANGER CONSTRUCTION

\begin{tabular}{|c|c|c|c|c|c|c|c|c|c|c|c|c|c|}
\hline \multirow[b]{2}{*}{ AI lo: } & \multicolumn{9}{|c|}{ Composition Limits (1) } & \multirow{2}{*}{\multicolumn{2}{|c|}{ Ochers }} & \multirow[b]{2}{*}{ Aluminum } & \multirow[b]{2}{*}{ Recommended Use in Heat Exchangers (2) } \\
\hline & silicon & Iron & Copper & $\begin{array}{l}\text { Man } \\
\text { ganese }\end{array}$ & $\begin{array}{l}\text { Mag- } \\
\text { nesium }\end{array}$ & Chrorider & Nicke] & $\underline{\text { Zinc }}$ & $\begin{array}{l}\text { Titan- } \\
\text { ium } \\
\end{array}$ & & & & \\
\hline 3003 & 0.6 & 0.7 & $\begin{array}{l}0.05- \\
0.20\end{array}$ & $\begin{array}{l}1.0- \\
1.5\end{array}$ & -- & -- & -- & 0.10 & - & 0.05 & 0.15 & Remainder & Heat excranger tube; baffles, flanges, stub ends \\
\hline 5052 & 0.25 & 0.40 & 2.10 & 0.10 & $\begin{array}{l}2.2- \\
2.8\end{array}$ & $\begin{array}{l}2.25- \\
2.35\end{array}$ & -- & 0.10 & -- & 0.05 & 0.25 & Remainder & fieat exchanger tube \\
\hline 5056 & 0.30 & 0.40 & 2.10 & $\begin{array}{l}0.05- \\
0.20\end{array}$ & $\begin{array}{l}4.5- \\
5.6\end{array}$ & $\begin{array}{l}0.05- \\
0.2 \%\end{array}$ & -- & 0.10 & -- & 0.05 & 0.15 & Remainder & Shells, header, water boxes \\
\hline 5083 & 0.40 & 0.40 & 0.10 & $\begin{array}{l}0.40- \\
1.0\end{array}$ & $\begin{array}{l}4.0- \\
4.9\end{array}$ & $\begin{array}{l}0.05- \\
0.25\end{array}$ & -- & 0.25 & $0 . \pm 5$ & 0.05 & 0.15 & Remainder & Shells, headers, water boxes \\
\hline 5454 & 0.25 & 0.40 & 0.10 & $\begin{array}{l}0.50- \\
1.0\end{array}$ & $\begin{array}{l}2.4- \\
3.0\end{array}$ & $\begin{array}{l}0.35- \\
0.22\end{array}$ & -- & 0.25 & 0.20 & 0.05 & 0.15 & Remainder & $\begin{array}{l}\text { Heat exchanger tube, shells, headers, water } \\
\text { boxes }\end{array}$ \\
\hline 6061 & $0.40-$ & 0.70 & $\begin{array}{l}0.15- \\
0.40\end{array}$ & 0.15 & $\begin{array}{l}0.8- \\
1.2\end{array}$ & $\begin{array}{l}0.04 \\
0.35\end{array}$ & -- & 0.25 & 0.15 & 0.05 & 0.15 & Renainder & $\begin{array}{l}\text { Heat exchanger tube; tie rods, flanges, } \\
\text { stub ends }\end{array}$ \\
\hline $\begin{array}{l}\text { Alclad } \\
3003 \\
\text { Core }\end{array}$ & Eame as & 3003 & & & & & & & & & & & Heat exchanger tube; baffles \\
\hline $\begin{array}{l}7072 \\
\text { cladding }\end{array}$ & $0.7 \mathrm{Si}+$ & $+\mathrm{Fe}$ & 0.10 & 0.10 & 0.10 & -- & -- & $\begin{array}{l}0.8- \\
1.3\end{array}$ & -- & 0.05 & 0.15 & Remainder & \\
\hline $\begin{array}{l}\text { Alclad } \\
\text { 6061 } \\
\text { Core }\end{array}$ & Same as & 5061 & & & & & & & & & & & Heat exchanger tube; tube sheets. \\
\hline
\end{tabular}

Notes: (1) Given in per cent maximum unless stated as a range.

Aluninum standards and data: The Aluminur Association.

(2) Alcoa Aluminum Heat Exchanger and Condenser Bcoklet. 
to pitting corrosion is of considerably more importance to the OTEC program than is resistance to crevice corrosion. Conditions that promote pitting corrosion cannot be controlled in OTEC while conditions that promote crevice corrosion can be controlled by (a) maintaining clean surfaces required for OTEC, and (b) designing OTEC heat exchangers to avoid crevices.

Data for specimens of Alclad 3003 from References 2 and 3 are recorded on pages 74,75 , and 76 in Table $I$ and pages 61,62 and 63 in Table II of the cataloged data. One group of Alclad 3003 specimens of unknown temper showed only minor pitting and crevice corrosion even after 36 months of exposure at a depth of 5640 feet. Another group of Alclad 3003 specimens in the H12 temper from similar exposures at the same test site showed uniformly deep pitting and crevice attack of 0.016 to 0.020 inch/year. Although, this difference in performance cannot be satisfactorily explained, it is unusual for alclad material of comparable quality. This leads the authors to suspect that the second series of specimens were prepared from substandard sheet. A detailed metallographic examination of typical specimens from each group might help clear up this point.

As mentioned above, the cataloged data do not appear adequate for the outright qualification of aluminum for OTEC. However, they do suggest that the aluminum alloys 5052 and Alclad 3003 are promising candidates that should be included in future tests where variables that may affect corrosion performance can be studied under conditions peculiar to OTEC.

VI. VARIABLES THAT AFFECT CORROSION

A number of variables that can affect the corrosion performance of aluminum and its alloys are given and discussed in the subsections that follow. A few of these have been so thoroughly studied and defined it can be stated categorically that they will have a harmful effect in sea water. On the other hand, the affect of others on performance have not been so clearly defined especially under OTEC conditions. OTEC is a new application for aluminum that involves a combination of conditions for which there has been no previous need for such corrosion data.

\subsection{Metallic Ions}

Metaliic ions that can affect the corrosion of aluminum are those of copper, lead, tin, nickel, cobalt and mercury. When these metallic ions are present in an electrolyte, surface and deep sea water, for example, they tend to deposit through electrochemical action onto aluminum. This is harmful to aluminum because it accelerates pitting corrosion. Since further tests will be required to qualify aluminum for OTEC, it is important that the experimental design specify uncontaminated sea water and exclude 
equipment of these metals. In the event that aluminum does qualify for OTEC the plant should be designed with no equipment containing these metals and their alloys upstream of the aluminum.

\subsection{Galvanic Corrosion}

In the galvanic series for metals in sea water aluminum is one of the more active (anodic) metals. Hence, there are many common metals and their alloys than can promote galvanic corrosion of aluminum when in metallic contact with it in sea water. Copper and copper containing alloys as well as steels including stainless steel will promote galvanic corrosion of aluminum in sea water. Corrosion of aluminum in a galvanic couple can be reduced to acceptable levels by cathodic protection or by providing complete electrical isolation between the aluminum and dissimilar metal of the couple. However, the best procedure is to design to avoid dissimilar metal couples. Therefore, if further evaluation of aluminum is made for OTEC, the experiments should be designed to avoid electrical contact between aluminum and dissimilar metals. Similarly, if aluminum does qualify for OTEC and is used for heat exchanger tubes, the tube sheets should also be of aluminum. Alclad plate such as Alclad (7072) 6061 is normaliy recommended for tube sheets. Water boxes or headers of aluminum construction would also be preferred although steel water boxes have been used provided they were (a) coated internally with a material such as Debecote (Debevoise Co., Brooklyn, N.Y.), or its equivalent, and (b) electrically insulated from the aluminum by using nonabsorbent gasket material and insulating sleeves and washers on fasteners.

\subsection{Crevice Corrosion}

Crevice corrosion is a localized form of attack that takes place when an electrolyte seeps into confined spaces such as crevices at faying surfaces, under absorbent gaskets, under scale, and beneath marine fouling. It results from a concentration gradient particularly of oxygen between the bulk of solution and the confined space. Aluminum and its alloys like many other common metals and alloys are susceptible to crevice corrosion.

None the less the authors restate their opinion that crevice corrosion of aluminum should not be a problem in OTEC. The degree of cleanliness mandated for OTEC heat transfer requirements should eliminate deposits of marine growth and scale as possible sources while design specifications can be formulated to minimize corrosion in crevices that cannot be avoided in heat exchanger construction. Preparation of design specifications might profit from further testing of crevice specimens to evaluate the benefit of various gasket materials and sealant compounds along with degree of mechanical tightness in alleviating crevice corrosion under conditions anticipated for OTEC. 
The effect of velocity on the corrosion of aluminum is not well documented in the literature. The results of work with high speed water wheels (48) are excluded as not being applicable to the problem at hand. Data collection in such tests may start at zero velocity but then jumps to a velocity in the neighborhood of $10 \mathrm{knots}$ or 4.9 to $5.2 \mathrm{~m} / \mathrm{sec}$ (16-17 feet per second) bypassing the range of interest. Godard and Booth (26) reported data from a three year test at Harbor Island, NC in which surface sea water at ambient temperature flowed continuously through aluminum pipe of CA-ls alloy (99.68 aluminum). They found pitting depth to decrease as the relative velocity of the sea water increased from 0.3 to $1.1 \mathrm{~m} / \mathrm{sec}$ (1 to 3.5 feet per second) and then increased with further increases in the relative velocity. They also observed that "marine growth", which was about $12.7 \mathrm{~mm}(1 / 2 ")$ thick at the lowest velocity, decreased with increasing velocity to become "non-existent" at velocities above $1.1 \mathrm{~m} / \mathrm{sec}(3.5$ feet per second). Their observation on "marine growth" and velocity is supported by Grey and Gashus (64) who state that "if sea water velocities are maintained above $0.9 \mathrm{~m} / \mathrm{sec}$ to $1.0 \mathrm{~m} / \mathrm{sec}$ (3 to $3.5 \mathrm{ft} / \mathrm{sec}$ ) "marine organisms" will have difficulty in attaching themselves and fouling is minimized".

Peterson and Lennox (34) report that minor variations in sea water velocity have marked effects on the corrosion of 6061-T6 aluminum. They found that a continuous flow at $0.23 \mathrm{~m} / \mathrm{sec}(0.75 \mathrm{ft} / \mathrm{sec})$ increased both pitting attack and corrosion of intentional crevices from that normaliy expected for 6061-T6 aluminum in quiescent sea water to an unacceptable level in 728 days.

Henrikson and Knutson (49) found that a velocity of $2.5 \mathrm{~m} / \mathrm{sec}$ $(8.2 \mathrm{ft} / \mathrm{sec})$ was too fast for aluminum tubes handling Baltic sea water at the temperature of $50^{\circ} \mathrm{C}\left(122^{\circ} \mathrm{F}\right)$.

Wilson and Hawkins $(65,66)$ cite two experiments conducted some years ago by the United States Navy. In one experiment at kure Beach, NC an aluminum heat exchanger containing Alclad (inside 7072) 3003 tubes handled surface sea water in the tubes continuously for two years at a velocity of $3.0 \mathrm{~m} / \mathrm{sec}$ ( 10 feet per second). The unit was then operated in a cyclical manner one month at velocity and one month with no flow. After a total exposure of 6 years the tubes were examined and although some local cladding consumption was observed no penetration into the 3003 core alloy was noted. In the other experiment sea water was pumped through pipe of several aluminum alloys for periods ranging from 12 to 42 months at a velocity of $3.0 \mathrm{~m} / \mathrm{sec}$ (10 feet per second). The aluminum alloys included in this experiment were 3003, Alclad (7072) 3003 and 5052. It was concluded that the life expectancy of both 3003 and Alclad 3003 pipe was at least 10 years. The 5052 pipe showed somewhat lower but acceptable resistance to sea water corrosion. Although these experiments do not show the effect of velocity on corrosion they do demonstrate that the aluminum alloys tested are capable of providing extended service handling surface sea water at a velocity in excess of that anticipated for OTEC heat exchangers. 
The authors suggest that corrosion data reported for deep waters in the Pacific $(2,3)$ and Atlantic $(7,8)$ Oceans may not be significant in the OTEC program. While it is true that a heat exchanger in the condensing cycle of an OTEC power plant will handle cold water from the ocean depths, the heat exchanger will not be at that depth. Instead, it will be closer to the surface. Deep water will rise in the intake pipe to enter the exchanger at a considerably lower pressure and perhaps a somewhat different chemical composition than at depth. Tube surfaces are also expected to be kept free of fouling in the OTEC application, thus minimizing any effect that slimes or other deep sea organisms might have on aluminum corrosion.

6.6 Oxygen

Oxygen can play a dual role in the corrosion of aluminum. In one role it serves to depolarize cathodic areas and thereby helps maintain the activity of pits once initiated. In the other role, it serves to maintain the integrity of the corrosion resistant oxide film on aluminum and thus reduce the tendency of pits to form.

Godard (9) believes that deaeration of a water is beneficial to aluminum. In experiments he performed, oxygen free water did not cause pitting of freshly etched aluminum, whereas, in oxygen saturated water the initiation of pits was rapid. He further cites a report by Rowe and Walker who found that in a water containing copper, bicarbonate, and chloride ions the exclusion of air reduced the amount of corrosion on aluminum to only one third of that encountered in the presence of air.

On the other hand, Fink and Boyd (15), using data obtained by Reinhart (3) from the NCEL Exposures in the Pacific off Port Hueneme, California, developed a plot that shows the depth of pitting for the 5000 series of alloys to increase as the oxygen content decreases from $5.25 \mathrm{ppm}$ in surface water to lower values at several depths. The word series is emphasized because it implies a number of alloy types each of which behaves somewhat differently than the other. The authors could not develop a plot as definitive as that of Fink and Boyd using Reinhart's data for 5052 aluminum alloy. It is worth mentioning here that a statistical test applied to Reinhart's data did not indicate a significant correlation between pit depth and the oxygen content of sea water.

In the NCEL program, Muroaka $(10,52)$ was investigating the effects of marine organisms on various materials and in one experiment he exposed specimens of an aluminum alloy under nearly sterile conditions in surface water and at depth. It is interesting to note that under the virtually sterile conditions he found the specimens to be affected about the same at depth as at the surface. 
Since the oxygen content of the deep water was less than that of surface water the implication is that oxycjen availability had no effect on corrosion in this test.

Evidence regarding the effect that oxygen content of natural sea water may have on the corrosion of aluminum is meager and inconclusive. A thorough investigation of this matter is recommended.

\subsection{Marine Organisms and Fouling}

A major difference between surface and deep ocean waters is the type of families of marine organisms that exist in each. Muroaka (52) reported very little "fouling" growth on materials exposed in the deep sea although hydroids and slime were found. Barnacles, sea squirts, bryozoa, mussels and so forth, found in shallow water exposures are not found in the deep sea.

Efird (45) investigated the relationships between corrosion and macro-fouling of metals in surface sea water at the International Nickel Company, Wrightsville Beach, NC test station. Tidal flow at this station is about $0.30 \mathrm{~m} / \mathrm{sec}$ ( 1 foot per second) and fouling is classified as moderate to heavy. He found that fouling properties of alloys are influenced by their corrosion characteristics and can be placed in one of the three following categories: (1) highly corrodible metals, (2) passive metals, and (3) toxic film forming metals. Highly corrodible metals such as carbon steel will foul and the fouling is easily removed with corrosion products, sloughing off at intervals during exposure. Passive alloys, he cites aluminum, foul readily and the fouling is tightly adherent. Toxic film forming metals such as copper and copper alloys are resistant to macro fouling. He reports that any effect of macro fouling on the corrosion of passive metals results from oxygen concentration cells where crevice corrosion takes place beneath the fouling organism. Haglwara (46) noted some mild corrosion beneath barnacle shells on alumi num alloys $1100,3003,5052,6063$ and 7075 exposed to surface waters off the coast of Japan. As pointed out previously in this report, all of the data cataloged were obtained under conditions of marine fouling and so include any effect such fouling may have on the corrosion of aluminum.

The effect of deep sea organisms on the corrosion of metals is not well documented. Willingham (16) studied the corrosion of metals by anaerobic bacteria in sea water under elevated hydrostatic pressure using cultures of sulfate reducers obtained from (1) carbonate sediments off Andros Island, B.W.I., (2) the Black Sea at 250 meters $(820 \mathrm{ft})$, and (3) the Black sea at 500 meters (1640 feet). He exposed specimens of ingot iron, electrical conductor grade aluminum and stainless steel to each culture at 20 and $40^{\circ} \mathrm{C}$ under pressures of $0,20.3$, and 60.8 mega pascals $(0,200$ and 600 atmospheres). The ingot iron was severely corroded at 0 and 20.3 mega pascals (0-20 atm.) but virtually 
unaffected at 60.8 mega pascals (600 atm). The aluminum and stainless steel specimens were virtually unaffected at all temperatures and pressures employed. He suggested that the cultures become sterile under 60.8 mega pascals (600 atm.) pressure because at that pressure the ingot iron was virtually unaffected. His findings with respect to aluminum corrosion by these organisms are in line with expectations. Hydrogen sulfide, a product of their metabolism, has no action on aluminum, whereas, its corrosive effect on iron and low carbon steels is well known. In regard to this reaction with iron it is suggested that if the iron sulfide corrosion products settle out on aluminum and remain undisturbed corrosion of aluminum can result from both concentration cell and galvanic action.

So far marine organisms have been considered solely with regard to their corrosive effect on metals. Of equal or perhaps more importance is the physical effect they can have on plant performance. Uninhibited growth of surface water organisms can lead to partial blockage of flow channels and even complete plugging of heat exchanger tubes. If slimes noted by Muroaka (52) form in OTEC condenser tubes handling water from the ocean depths, heat transfer efficiency could be seriously impaired even though little or no change in total flow occurred.

It has been pointed out previously that macro fouling will be minimized if operating velocities are maintained above 0.9 to $1.0 \mathrm{~m} / \mathrm{sec}$ ( 3 to 3.5 feet per second). If operation is intermittent so that velocities fall below this range large marine organisms can attach themselves and flourish when velocities are raised due to the increased rate of food supply. In this case chlorination may be beneficial. The Alcoa desalination pilot plant evaluated at San Juan, Puerto Rico was operated intermittently for a period of two years during which chlorination at the level of $0.75 \mathrm{ppm}$ chlorine proved beneficial in maintaining clean heat transfer surfaces. Weight (68) has also used chlorination to prevent marine growth in an ocean water cooling system for a power station located on the west coast. Turner (69) reported that continuous chlorination at levels as low as $0.25 \mathrm{ppm}$ residual chlorine prevented attachment and growth of slime bacteria as well as macro-organisms in sea water piping system. In the authors experience chlorination at the levels indicated should not have a harmful effect on the performance of aluminum and none would be expected under OTEC conditions.

A method patented by Turner (70) for preventing marine fouling involves treating submerged surfaces with water containing ions generated by electrolysis from anodes of aluminum, iron or copper containing from 0.25 to $6 \%$ of arsenic and/or antimony. Copper anodes, of course, would not be used with aluminum equipment. 
Blogoslawski (71) et al, describe an ozone water treatment that eliminated troublesome fouling associated with piping large volumes of sea water. The effect of ozonation on the performance of aluminum in sea water is not known to the authors.

\subsection{Cathodic Protection and Antifouling Paints}

It is expected that with OTEC plants partly submerged at sea free use will be made of cathodic protection as well as antifouling paints to mitigate corrosion and marine fouling of exterior metal surfaces.

Cathodic protection of a single metal system whether steel or aluminum would be a relatively simple matter. However, if tube sheets and headers of the OTEC power modules are aluminum, as they should be with aluminum tubes, and other components are steel, the problem becomes somewhat more complicated because the exterior surfaces of both the aluminum and steel would require protection. Either an impressed current system or sacrificial anodes could be used and at this stage of OTEC development it is virtually impossible to say which would be the better choice. Both systems are used on the Alcoa Seaprobe (73). The impressed current system would permit finer control of the cathodic protection process than would be possible with sacrificial anodes which is important when protecting a combination of dissimilar metals, but it is also considerably more expensive. If sacrificial anodes are decided upon, those containing tin or mercury (36) as alloying elements should be avoided if there is a possibility for corrosion products from them to settle or deposit on aluminum equipment. The corrosion products would contain tin and mercury in substantial amounts both of which can promote corrosion of aluminum. Mercury can also amalgamate with aluminum as well as some other common metals to cause corrosion that can be catastrophic.

Antifouling paints would likely be considered for submerged parts of OTEC plants to minimize marine growth. Paints of this kind containing tributyl tin oxide (35) would be preferred where aluminum is concerned over those containing copper compounds as the toxic agent. Antifouling paints containing mercury compounds should not be used on or near aluminum equipment

6.9 Calcium

Data related to calcium scaling by sea water is scarce. However, both deep and surface water are essentially saturated at their respective $\mathrm{pH}$, temperature and pressure with calcium, sulfate and bicarbonate ions. Hence, the potential for scaling exists when, for example, deep water is brought to the surface. Whether 
scaling would occur in OTEC power plant condenser tubes depends on kinetic factors, availability of nucleation sites, operating parameters of the plant and sea water composition at a specific location and depth. It would be worthwhile to investigate this potential problem through a suitably designed research program.

6.10 Tube Vibration

Forces that cause tubes to vibrate may result from any one or combination of (1) high vapor velocity, (2) flow of fluid over flexible tube, (3) pumping operations, and (4) expansion and contraction of tubes due to thermal conditions within the exchanger. Vibration damage, of course, is a mechanical factor which Sebald (67) attributes to improper design. He analyzed the problem concluding that a reduction in span between baffles will increase the frequency but reduce the amplitude of vibration resulting in an increase in stiffness of the tube. He states that this relationship is of fundamental importance in designing a condenser to eliminate damaging tube vibration and demonstrates the method of applying the relationship to condenser design.

The authors agree that Sebald's solution should reduce the tendency for condenser tubes to fail by fatigue and/or corrosion fatigue, but so far as it is known this mode of failure has rarely if ever been encountered with aluminum condenser tubes. However, fretting has been found on tubes that vibrated or rubbed against baffles that could in time contribute to failure by fatigue. This type of damage as encountered in a steam plant surface condenser and illustrated in Figure 1 is the reason for concern about tube vibration in OTEC heat exchangers. The question of whether such damage will develop under OTEC conditions needs to be addressed. The arbitrary use of preventative measures such as the installation of sleeves on tubes where they pass through baffles would be an expensive procedure if it were not needed.

\subsection{Vapor Impingement}

Vapor impingement is mentioned because it is a common source of localized danage to aluminum tubes in steam plant surface condensers that perform the same basic function expected of OTEC power plant condensers. That function is to condense a low pressure vapor leaving a turbine - generator set.

In steam plant service the turbine exhaust steam entrains droplets of condensate and this wet steam impinging on aluminum tubes at local velocities well in excess of the average velocity can promote damage typical of that illustrated in Figure 1 . 


\section{Figure I}

Aluminum tube from a steam plant surface condenser illustrating:

(1) Fretting damage where the tube passed through a baffle.

(2) Severe damage that resulted from impingement of wet steam. 


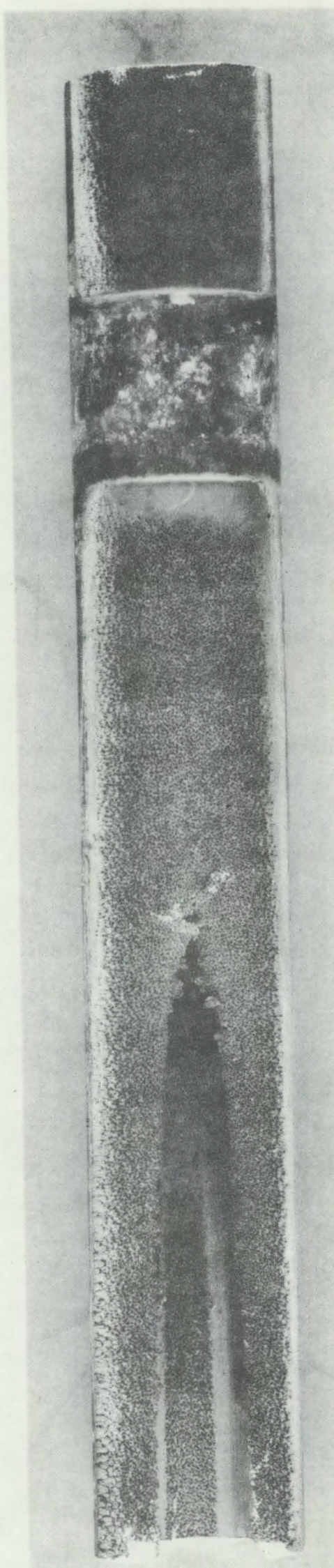

1. FRETTING

\section{IMPINGEMENT}

Figure 1 
While designs for OTEC power plant condensers call for lower average vapor velocities than in steam plant surface condensers, care needs to be exercised to avoid local velocities well in excess of the average velocity. If these exist, then the effect of ammonia vapor containing droplets of liquid impinging on aluminum tubes should be addressed.

\section{$\underline{6} .12$ Tube Cleaning}

Very little information is available concerning cleaning of aluminum condenser tubes that would be of value in the OTEC program. Manual methods that have been used successfully with aluminum tubes are briefly described in the Alcoa Aluminum Heat Exchanger and Condenser Booklet (72), but such methods would not be practical for OTEC where continuous on line cleaning would be needed to maintain the degree of cleanliness required for heat transfer.

Continuous on line cleaning methods for heat exchanger and condenser tubes are available from the American M.A.N. Corporation, 1114 Avenue of the Americas, New York, N.Y. and the Amertap Corporation, Mineola, New York. During March, 1977 each company was contacted regarding the use of the methods with aluminum tubes. Mr. Gerhard Foerster responding for the American M.A.N. Corporation stated that to the best of his knowledge the M.A.N. system had never been used with aluminum. A similar response was received from Mr. M. J. Gruen of the Amertap Corporation in regard to the Amertap process. Both respondents provided literature describing theri respective process in some detail.

The M.A.N. process (74) uses brushes with bristles of selected plastic held in place by titanium wire. Each condenser tube is serviced by its own brush and has a manually installed plastic cage at each end in which the brush is parked between cleaning cycles. Cages and brushes are available for all common tube sizes. The brushes are moved from one end of the tube to the other by reversing the direction of cooling water flow. A complete cleaning cycle for a large installation requires about 80 seconds. An automatic timer permits intervals between cleaning cycles to be matched to the degree of water pollution. The brushes, resistant to sea water attack, have a life of over 10,000 cycles and then would have to be replaced which could be a major undertaking for an OTEC condenser. However, the M.A.N. brushes should be well suited for cleaning tubes with fluted water side surfaces. Since the use of fluted tubes in OTEC power plant heat exchangers is a distinct possibility, the abrasive action of M.A.N. brushes on aluminum tubes should be investigated. 
The Amertap process (75) employs plain sponge rubber balls that are constantly circulated through the condenser tubes. The balls are slightly larger in diameter than the tubes and are compressed as they travel the length of the tube. The rubbing action keeps the tube walls clean and free from deposits of all types. Suspended solids are kept moving, while bacterial slimes and marine organisms are wiped quickly away. The balls are selected to provide maximum cleaning for a particular installation. Their specific gravity is nearly equal to that of the cooling medium resulting in a homogeneous distribution throughout the cooling medium. Balls are available in several degrees of resiliency depending on the requirements.

An abrasive coated ball is also available for situations where condenser tubes have already been heavily fouled. The degree of abrasiveness can be varied to meet the degree of fouling. The effect is a gentle scouring of the tube wall, removing scale slowly but steadily until the tube is ready to be maintained by the normal plain sponge rubber balls.

The balls are circulated in a closed loop that includes the condenser. At the discharge end they are caught in a screening device installed directly in the pipe line. They are then routed through a collector unit back to the inlet ball injector nozzles. This insures a uniform distribution of the balls over the pipe cross-section.

The number of balls required is a function of the number of tubes in the condenser. When properly charged, i.e. the correct number of balls inserted, each tube receives a ball on the average of once every five minutes.

The cleaning cycle can be interrupted at any time at the collector unit to count the balls, check sizes, or take them out of service. Wear eventually reduces the size of the balls and whenever they reach a predetermined diameter they should be replaced. Replacement of balls can be easily and quickly done at the collector unit.

The authors are aware that the Amertap process has been suggested for OTEC and there is no reason why plain sponge rubber balls could not be used to advantage with aluminum tubes. Abrasive coated rubber balls could be a problem and their affect on aluminum tubes should be determined. 
6.13 Ammonia-Sea Water Solutions

Depending on the design of OTEC Power Plant heat exchangers the possibilities of ammonia leakage into sea water and of sea water leakage into ammonia exist. Included in problems that might develop from such leakage is corrosion of aluminum tubes by both dilute ammonia-sea water solutions and ammonia contaminated with sea water. Previous experience proves that aluminum alloys are attacked by aqueous ammonia solutions. However, when conditions permit passivation of the aluminum surface corrosion generally proceeds at a very low rate after an initial period of rapid attack. Bonewitz (78) reported initial corrosion rates on the order of $25 \mathrm{~mm}$ per year $(1000 \mathrm{mils} / \mathrm{yr})$ for aluminum alloys in dilute solutions of ammonia in deionized water. Since leak detection in an OTEC Power plant heat exchanger would hardly be instantaneous and since passivation of aluminum tubes could be retarded or even prevented by both velocity effects and continuous cleaning, data on the compatibility of aluminum with ammonia-sea water solutions is needed. Such data for aluminum in solutions of ammonia and actual sea water are scarce. However, Bonewitz $(76,78)$ investigated the performance of the aluminum alloys 1100, 3003, 5052, and 6063 in initially aerated solutions of ammonia and synthetic sea water under quiescent conditions and the 3003 alloy under conditions of flow using velocities of 1.2 meters per second (4 ft/sec) and 2.4 meters per second ( $8 \mathrm{ft} / \mathrm{sec})$.

Under the quiescent conditions synthetic sea water containing up to $30 \%$ by weight of ammonia caused uniform corrosion of the aluminum specimen, characterized generally by initial rates of attack no higher than $0.5 \mathrm{~mm}$ per year $(21 \mathrm{mils} / \mathrm{yr}$ ) followed by decreased corrosion rates or passivation of the metal surface. Selecting synthetic sea water containing 5\% ammonia that caused the highest initial corrosion rate as the most aggressive solution, corrosion rates for 3003 tube were found to decrease as the water velocity was increased up to 2.4 meters per second $(8 \mathrm{ft} / \mathrm{sec})$.

In synthetic sea water containing from $30 \%$ up to 708 by weight of ammonia only superficial etching of the metal surfaces occurred. Rates of attack based on weight loss in these solutions were no greater than $.013 \mathrm{~mm}$ per year $(0.5 \mathrm{mil} / \mathrm{yr})$.

In solutions containing from $90 \%$ up to $100 \%$ of ammonia pitting of the aluminum occurred. The depth of pitting increased as the water content decreased from $10 \%$ (908 amuonia) to $0 \%$ (1008 antnonia), but in no case was it greater after four weeks of exposure than after one week. Maximum pit depths measured by metallographic methods on the aluminum alloy specimens after four weeks of exposure to $100 \%$ ammonia were reported as follows: 
$\underline{\text { Alloy }}$

1100

3003

5052

6063
Maximum Pit Depth

\begin{tabular}{lc} 
mm & mils \\
\cline { 3 - 3 } .041 & 1.6 \\
.119 & 4.7 \\
.058 & 2.3 \\
.015 & 0.6
\end{tabular}

The lower corrosion rates in dilute ammonia-synthetic sea water solutions as compared to those reported for dilute ammoniadeionized water solutions is attributed to the presence of magnesium ions in the sea water. The magnesium reacts with hydroxyl ions to concurrently precipitate magnesium hydroxide and lower the $\mathrm{pH}$ of the solution resulting in low initial corrosion rates. The magnesium also acts as a corrosion inhibitor by helping to establish a passive film on the aluminum surface. The integrity of the passive film under conditions of flow is being investigated further by Dow Chemical Company under a contract with Battelle Pacific Northwest Laboratories for DOE.

The authors call attention to the chemistry of ammonia-sea water solutions, resulting in precipitation of magnesium hydroxide, as possibly a greater potential hazard to OTEC Power Plant performance than corrosion of aluminum tubes. Magnesium hydroxide precipitating in the tubes as a result of ammonia leakage into sea water would be removed by whatever continuous on line cleaning method was used. However, continuous cleaning of the outside (ammonia side) of the tubes is not practical and precipitate resulting from sea water leakage into ammonia would tend to accumulate on the tubes. This would add a substantial resistance to heat transfer and reduce the overall efficiency of the plant.

\section{SERVICE EXPERIENCE}

A number of service histories selected with the application of aluminum tubes in OTEC in mind are given in Apepndix III. Information on operating conditions such as water velocity, water temperature and water treatment is included for the few cases where such data were available. Most of these histories involved the use of Alclad (7072) 3003 aluminum tubes handling sea. water because this alloy has for years been the heat exchanger tube alloy recommended for handling aggressive waters. In more or less recent service tests nonclad aluminum alloy tubes have been evaluated in desalination pilot plants. At both the osw test bed site at Freeport, Texas $(40,41,42,63)$, and the Reynolds/ OSW Wrightsville Beach (63) facility the nonclad tubes performed exceptionally well handling treated sea water. The treatment involved for the most part removing noncondensibles $\left(\mathrm{CO}_{2}, \mathrm{O}_{2}\right.$, etc.) from the sea water. At the osw test bed site at Freeport, Texas 
$(41,63)$, the heat reject exchanger \#21 handled raw sea water in the nonclad tubes. During the first six months of service the nonclad tubes were virtually unaffected. However, during six additional months replacement tubes as well as remaining original tubes were seriously pitted and perforated beneath barnacle shells.

The service histories for Alclad (7072) 3003 tube suggest that a service life well in excess of 10 years might be expected handling surface sea water. Any effect that continuous cleaning might have on the corrosion performance of Alclad (7072) 3003 tube must be determined by experiment.

\section{CONCLUSIONS}

Care must be taken in using the cataloged data for qualifying aluminum tubes for OTEC power plant heat exchangers because these data do not include the effects of velocity and continuous cleaning that will exist in an OTEC plant. However, there is evidence in the cataloged data and elsewhere in the report that support the following premises each of which require further study before a firm decision can be made to qualify aluminum tubes for OTEC heat exchangers.

(1) The aluminum alloys 5052 and Alclad (7072) 3003 appear promising for OTEC heat exchanger tubes. Tubes of each alloy should be included in future tests to determine their performance under conditions peculiar to OTEC.

(2) The effect of depth and the related oxygen depletion on the performance of aluminum in sea water is not well established by the existing data. Depth alone may not be significant because the chemical composition of water entering the tubes of an OTEC power plant condenser could be somewhat different after rising through the intake pipe than it was at depth. The oxygen content could be the same or somewhat greater than at depth depending on the intake design. In view of the uncertainties involved the effect of deep water and oxygen content on the performance of aluminum should be thoroughly examined under conditions anticipated for an OTEC power plant condenser.

(3) The existing data do not show to what extent crevice corrosion would be encountered on aluminum in OTEC power plant heat exchangers. OTEC heat exchangers should be designed to avoid crevices. 
(4) The effect of sea water velocity under the conditions and in the range anticipated for OTEC on the performance of aluminum is not well documented in the literature. Alcoa recommends water velocities no greater than $7 \mathrm{ft} / \mathrm{sec}$. for aluminurn heat exchanger tube. In the author's opinion localized conditions that pronote turbulence well in excess of that expected for the average velocity are more significant than is the average velocity alone.

(5) The effect of cleaning on the corrosion performance of aluminum tubes in an OTEC power plant heat exchanger cannot be determined from existing data. Chlorination at levels no greater than $0.75 \mathrm{ppm}$ chlorine should be beneficial in reducing marine fouling and have no adverse effect on the corrosion of aluminum tubes. The effect of abrasive action inherent to continuous on line cleaning with either sponge rubber balls or brushes is not known and needs to be determined.

IX. OTHER AREAS FOR INVESTIGATION

In addition to the above more or less basic considerations the author suggests investigations in the following areas.

Calcium

Data relating calcium content of sea water to scaling tendencies is scarce. Typical data on calcium scaling should be developed through a suitably designed research program. If calcium scaling turns out to be a problem, the Amertap and M.A.N. processes should be investigated as a means of reducing it.

Tube Vibration

The effect of vibration on tube performance should be examined under conditions that duplicate as closely as possible those anticipated for OTEC condensers. This means the specimen tube should be the same length, diameter and wall thickness expected for OTEC. The specimen tube should be fixed at both ends while baffles should be the same thickness and spaced as they would be in service. The sea water velocity as well as the inside and outside tube wall temperatures should duplicate the values expected in service. The concern here is not primarily fatigue but whether fretting between the tube and baffles will cause serious darnage. 
Vapor Impingement

The possibility of damage to aluminum tubes by impinging ammonia vapor containing droplets of liquid ammonia should be addressed under conditions anticipated for OTEC.

RHW : RAB : ali 
BIBLIOGRAP HY

Literature Sources

1. Southwell, C. R. et. al. "Corrosion of Metals in Tropical Environments ", Materials Protection, Vol. 15, No. 7, July, 1976.

2. Jenkins, J. F, and Reinhart, F. M., "Corrosion of Metals in Hydrospace", Corrosion/74, Paper NO. 82.

3. Reinhart, F. M., "Corrosion of Metals in the Deep Ocean", Civil Engineering Laboratory, Naval Construction Battalion Center, Port Hueneme, CA, February, 1976.

4. Ailor, W. H., Jr., "Aluminum Alloys After Five Years in Sea water", Materials Performance and the Deep Sea ASTM STP 445, American Society for Testing and Materials, 1969.

5. Czyxyca, E. J., et. al., "Corrosion of Aluminum Alloys in Exfoliation Resistant Tempers Exposed to Marine Environments for Two Years", Naval Ship Research and Development Center, Annapolis, Maryland, November, 1974, NTIS, AD/A - 002234.

6. Lindgren, S. et. al., "Weldable Structural Aluminum Alloys Corrosion Resistance in Outdoor Atmospheres and in water", 7 th Scandinavian Corrosion Congress, May 26-28, 1975, Trondheim.

7. Wallace, B. L., and Collett, W. A., "Evaluation of Materials Exposed for One Year on a Deep Sea Array in the Tongue of the Ocean, Bahamas", Naval Research and Development Laboratory, Annapolis, Maryland, Report 9-15, October, 1970.

8. Third Interim Report of Progress NRL Memorandum Report 1634 , July, 1965, Edited by T. J. Lennox, Jr., pp. 47-55.

9. Goddard, H. P., et. al., "The Corrosion of Light Metals, Wiley, 1967.

10. Muroaka, J. S., "Relationship Between Marine Fouling and Corrosion Rate of Carbon Steel and Aluminum Alloy at the Surface and at 6000 Feet", Technical Report R681, May, 1970, Naval Civil Engineering Laboratory, Port Hueneme, Ca.

11. Deluccia, J. J. and Taylor, E., "Evaluation of Metallic Materials Exposed to the Deep Ocean Environment at 5640 Feet", Aeronautical Materials Laboratory Report No. NAEC-AML-2132, June, 1965. 
12. Reinhart, F. M., "Preliminary Examination of Materials Exposed on STUl-3 in the Deep Ocean", U.S. Naval Civil Engineering Laboratory, Port Hueneme, Ca., Technical Note N-605, June, 1964.

13. Wie, M. W., "Experience with Alclad Aluminum in a Deep Sea Buoyancy Sphere", Corrosion, Vol. 23, 1967.

14. Brown, B. F., et. al., "Corrosion and Cathodic Protection of Underwater Surveillance Systems - A General Review of the Problem", U. S. Naval Research Laboratory, Washington, D.C. NRL Memorandum Report 1436, June, 1963.

15. Fink, F. W., and Boyd, W. K., "The Corrosion of Metals in Marine Environments" Defense Materials Information Center, Battelle Memorial Institute, Columbus, Ohio, DMIC Report 245.

16. Willingham, Charles A., "Anaerobic Bacterial Corrosion of Metals in Sea water at Elevated Hydrostatic Pressures", Williams F. Clapp Laboratories, Inc., Battelle Memorial Institute, Duxbury, Mass. to U. S. Department of the Navy, Office of Naval Research, Microbiology Branch, ONR Contract No. N00014-68-C-0365 Final Report, July 31, 1971.

17. Reinhart, F. M., "Corrosion of Materials in Surface Sea Water After 6 Months of Exposure", Naval Civil Engineering Laboratory, Port Hueneme, Ca., Technical Note N-1023, March, 1969.

18. Williarns, J. S. and Hodgsen, A. S., "Multistage Flash Distillation Unit Utilizing Diesel Generator Waste Heat", Naval Facilities Engineering Command, Naval Civil Engineering Laboratory, Port Hueneme, Ca., Technical Report R-595, September, 1968 .

19. Ailor, W. H., Jr., "Ten Year Sea Water Tests on Aluminum", Corrosion in Natural Environments ASTM STP 558 American Society for Testing and Materials, 1974, pp. 117-134.

20. Ailor, $W$. H., Jr., "A Review Paper - Aluminum in Desalination" Naval Engineers Journal, October, 1975, pp. 69-83.

21. Ailor, $\boldsymbol{W} . \mathrm{H}$. Jr., "Evaluation of Aluminum Panels Exposed in the Pacific Ocean at 2340 Feet for Six Months", Metallurgia, March, 1967.

22. Ailor, W. H. Jr., and Lindberg, R. I., "Corrosion Resistance of Aluminum Alloy 5083 in Marine Environments", Journal of the American Society of Naval Engineers, Vol. 73, No. 3, August, 1961 .

23. Ailor, W. H. Jr., "Evaluation of Aluminum After one Year Deep Sea Exposure", Journal of Hydronautics, Vol. 2, No. 1, January, 1968, pp. 26-33. 
24. Ailor, W. H., Jr., and Reinhart, F. M., "Three-Year Tidewater Corrosion of Aluminum Alloys", Naval Engineers Journal, June, 1964.

25. Ailor, W. H. Jr., "Flowing Sea Water Corrosion Potentials of Aluminum Alloys", NACE 26th National Conference, March 2-6, 1970, Philadelphia, Pa., Preprint No. 36.

26. Goddard, H. P., and Booth, F. F., "Corrosion Behavior of Aluminum Alloys in Sea Water", Travaux du Centre de Recherches et d' Etudes Oceanographiques, Vol. 6, 1965, pp. 37-52 (English).

27. Fouth, D. A. and Lindberg, R. I., "Aluminum Heat Transfer Surfaces-Multistage Flash Distillation Plant", OSW Research and Development Report No. 583, June, 1970.

28. Boyd, W. K., "State of the Art Report on Corrosion in Deep Ocean and Related Environments", Battelle Memorial Institute Columbus, Ohio, May 14, 1964.

29. Ostrozynski, R. L. and Jones, P. E., "The Prevention or Biofouling on Heat Transfer Surfaces of Ocean Thermal Energy Converters", Allied Chemical and Dye Corp. to U. S. ERDA Contract No. E(11-1)-2672 Progress Report, May 1, 1975 November 30, 1975, issued December, 1975.

30. Kumim, E. L., "Heat Transfer and Corrosion Characteristics of Capillary Fluted Aluminum Tubes", Progress Report No. 103, Air Research Manufacturing Co. Division of Garrett Corp., for OSW, October, 1964.

31. Vargel, C. and Pellectier, M., "Aluminum Alloys in Sea Water Desalination Installations", 3rd International Symposium on Fresh water From the Sea, Vol. 1, 1970, Dubrovnik, Yugoslavia, September $13-17,1970$.

32. Legault, R. A., "Seawater Corrosion Control by Environment Modification", The Dow Chemical Co., Freeport, Tecas, for OSW, Contract No. 14-01-0001-1482, Report No. 438, April, 1969.

33. Groover, R. E., et. al., "Characterization of the Corrosion Behavior and Response to Cathodic Protection of Nineteen Aluminum Alloys in Sea Water", Naval Research Laboratories, Washington, D.C., NRL Memorandum Report 1961.

34. Peterson, M. H. and Lennox, T. J., "The Effects of Exposure Conditions on the Corrosion of 6061-T6 Aluminum in Sea Water", Corrosion/76 March 22-26, 1976, Houston, Texas, Paper No. 187. 
35. Lennox, T. J., et. al., "The Effects of Dissimilar Metals and Toxicants from Antifouling Paints on the Corrosion of 5086 and 6001 Aluminum Alloys and Their Response to Cathodic Protection", Naval Research Laboratories, Washington, D.C., NRL Memorandum Report 2444, May, 1972.

36. Lennox, T. J., et. al., "The Effect of Mercury and Tin from Aluminum Galvanic Anodes on the Corrosion Characteristics of 5086-H34 and 6061-T6 Aluminum Alloys in Sea Water", Naval Research Laboratoires, washington, D.C.

37. Groover, R. E., et. al., "Cathodic Protection of Nineteen Aluminum Alloys Exposed to Sea Water Corrosion" Materials Protection and Performance, Vol. 8, No. 11, November, 1969.

38. Legault, R. A. and Bettin, W. J., "Performance of Aluminum Alloys Under Desalination Conditions", Materials Protection and Performance, Vol. 10, No. 8, March, 1971.

39. Desai, M. N., et. al., "Corrosion Inhibitors for Aluminum and Aluminum-Based Alloys - Part I", Anti Corrosion, Vol. 18, No. 4, April, 1971.

40. Verink, E. D. Jr., "Dynamic Materials Testing of Aluminum Alloys for Desalination Service", Materials Protection adn Performance, Vol. 8, 1969.

41. Verink, E. D. Jr., and George, P. F., "Long Term Tests of Aluminum Alloys for Desalination Service" Corrosion/73 Paper No. 58.

42. Verink, E. D. Jr., "Aluminum Alloys Resist Corrosion in Desalination Environment", Materials Protection and Performance, Vol. 12, 1973.

43. Person, N. I.,, "Fatigue Properties of Pre-Corroded Aluminum Sheet Alloys", Materials Performance and Protection, Vol. 14, 1975, pp. 22-26.

44. Chu, H. P., "Fatigue Crack Propagation in a 5456-Hll7 Aluminum Alloy in Air and Seawater", Journal of Engineering Materials and Technology.

45. Efird, K. D., "The Interrelation of Corrosion and Fouling of Metals in Sea Water", Materials Performance and Protective, Vol. 15, No. 4, April, 1976.

46. Hagiwara, Michiki, "studies on the Corrosion of Surface Treated Aluminum Alloys in Sea Water", Journal of Japan Institute of Light Metals, Vol. 19, No. 4, April, 1969 (In Japanese, Abstract in English). 
47. Flournoy, R. W., "The Corrosion Performance of Aluminum Transportation Equipment", Metals Engineering Quarterly, vol. 8, No. 3, August, 1969.

48. Davis, J. A., and Gehrin, G., "The Effect of Velocity on the Sea Water Corrosion Behavior of High Perofmrance Ship Materials", Materials Performance, Vol. 14, 1975, pp. 32-39.

49. Henrikson, S. and Knutson, L., "Corrosion Tests in Baltic Sea Water on Heat Exchanger Tubes of Various Metallic Materials", British Corrosion Journal, Vol. 10, No. 3, 1975, pp. $128-135$.

50. Compton, K. G. et. al., "A Comparison of Corrosion Rates Determined by Linear Polarization Measurements and Weight Loss Determinations for a Wide Variety of Metals Exposed to Flowing Sea Water for Two Years", Corrosion/74 Paper No. 120 .

51. Cambel1, H. S., "The Compromise Between Mechanical Properties and Corrosion Resistance in Copper and Aluminum Alloys for Marine Applications", Ocean Engineering, Vol. 1, 1969, pp. 387-397, Pergamon Press.

52. Muroaka, J. S., "Effect of Deep Ocean Organisms on Construction Materials", Proceedings of the First Joint Aerospace and Marine Corrosion Technology Seminar (NACE) July 10-12, 1968, Century Plaza Hotel, Los Angeles, Ca.

53. Laque, F. L., "Deterioration of Metals in an Ocean Environment", Ocean Engineering, Vol. 1, 1969, Pergamon Press.

54. Rynewicz, J., "Marine Corrosion Control for Deep Submergence Vehicles", Proceedings of the Air Force Materials Laboratory Fiftieth Anniversay Technical Conference on Corrosion of Military and Aerospace Equipment, Denver, Col, May 23-25, 1967, Paper No. A-69.

55. Booth, F. F., "Corrosion of Joints in Aluminum and AluminumSteel Structures", British Corrosion Journal, Vol. 2, March, 1967. pp. 55-68.

56. Whitted, A. L. and Schrieber, C. T., "Some Effects of Altered Sea Water Chemistry or Materials Performance in Desalination Plants", American Institute of Chemical Engineers Symposium Series No. 129, Vol. 69, 1973, pp. 508,514.

57. Lindgren, A. S. and Mattson, E., "Corrosion Resistance of Some Welded Aluminum Alloys in Sea Water", 5 th Scandinavian Corrosion Congress, October 7-10, 1968, Vol. 2, Paper, No. 32 . 
58. Sutton, Frank, "Corrosion and Stress Corrosion Properties of Non-Heat Treatable Aluminum Alloys in Marine Environments" Research and Development Report 910032 Subproject S-R0070102 June 12, 1967, U.S. Naval Experiment Station, Annapolis, Maryland.

59. Iskra, Ye.V., et. al., "Corrosion and Protection of Sea Going Ships", Sudostroyenine Publishing House, Leningrad, 1973, Naval Intelligence Support Center, washington, D.C., September, 1974 .

60. Ailor, W. H. Jr., "Aluminum in Ocean Engineering", Reynolds Metal Co., Metallurgical Research Division Presented at UCIA Materials for Ocean Engineering short Course in Los Angeles, October 11, 1967.

61. Alcoa Internal Report, SS Alcoa Clipper.

62. Alcoa Internal Data, SS Alcoa Seaprobe.

63. Desalination Materials Manual Dow Chemical Co. for Office of Water Researci and Technolgoy, May, 1975.

64. Gray, T. J., anä Gashus,o. K., "Tidal Power", Proceedings of an International Conference on Utilization of Tidal Power, May 24-79, 1970, at the Atlantic Industrial Research Inst. Nova Scotia Technical College, Halifax, Plenum Press, 1972, p. 538 .

65. Wilson and Hawkins, "Aluminum Alloys in Nuclear Reactor Systems; Sea Water Coolants", Prepared by ACF Industries for Reactor Centrnm Nederlands, Hague, Nederlands, 1957 (Original Source Reynolds Aluminum Co.).

66. Wilson and Hawkins, Ibid, COriginal Source U.S. Navy Department Engineering Experiment Station Report No. 040037A(5)).

67. Sebald, Joseph F., "Control of Vibration in steam Surface Condensers", Gilbert Associates Inc., Reading, Pa., 19603 Presented at Seminar on Performance of Surface Condensers in Nuclear and Fossil Power Plants at the Ohio State University, Columbus, Ohio, June 2-4, 1975.

68. Weight, R. H., "Ocean Cooling water system for 800 MW Power Station", J. Power Division Proceedings of the American Society of Civil Engineers, Puber December, 1958.

69. Turner, H. J. et. al., "Chlorine and Sodium Pentachlorophenate as Fouling Preventives in Sea water Conduits", Ind. and Engineering Chemistry, Vol. 40, No. 3, March, 1948. 
70. Turner, Roy V. et al, "Marine Antifouling", British Patent No. $1,147,54,1969$ April.

71. Blogoslawski, Walter J. et al, "Ozone Disinfection of a Seawater Supply System", International Symposium on Ozone for Water and Waste Water Treatment, lst Proceedings, Washington, D.C. 1973 December 2-5, pp. 674-687, Published by International Ozone Institute Inc., Waterbury, Conn. 1975.

72. Alcoa Aluminum Heat Exchangers and Condensers Published by The Aluminum Company of America, Pittsburgh, Pa.

73. Horst, R. L. Jr., and English, G. C., "Cathodic Protection of the Alcoa Seaprobe", Alcoa Internal Report, 1969 June 18, Revised 1974 August 15.

74. "On-Load Cleaning System for Condensers and Tubular Heat Exchangers", American M.A.N. Corporation, 1114 Avenue of Americas, New York, New York 10036.

75. "Factors in Increasing Heat Exchanger Efficiency", an Engineering Staff Report by Amertap Corporation, 1501 Franklin Avenue, Garden City, New York.

76. Bonewitz, R. A., "Concurrent Studies of Enhanced Heat Transfer and Materials for Ocean Thermal Exchangers", ERDA Contract No. E(11-1)-2641, Carnegie-Mellon/Alcoa Subcontract No. 1-53500 Final Report 1976 October 29.

77. Pachter, M., Barak, A. and Weinberg, J., "Reducing Costs by Using Aluminum Tubes in Multi-Effect Desalination Plants", Developments in Desalination Technology in Israel Proceedings of the Eighth National Symposium on Desalination en bogeq 1971 March 14-15, National Council for Research and Development, Prime Ministers Office, Jerusalem.

78. Bonewtiz, R. A., "The Performance of Aluminum Alloys in Ammonia-Sea Water Solutions", Fifth Ocean Thermal Energy Conversion (OTEC) Conference, Miani, Florida, 1978 February 20-22. 
APPENDIX I

OUTSIDE SOURCES CONSULTED 
Government Laboratories

Naval Research Laboratories

Washington, D.C.

Dr. M. H. Peterson

Naval Civil Engineering Laboratory

Port Hueneme, CA

Fred M. Reinhart (Ref.)

National Bureau of Standards

Washington, D.C.

Dr. Jerome Kruger

Universities

University of Florida

College of Engineering

Dept. of Metallurgical \& Materials Engineering

Gainesville, FL

Dr. E. D. Verink, Jr.

University of Miami

School of Marine and Atmospheric Sciences

10 Rickenbacker Causeway

Miami, FL

Dr. H. V. Craig, Jr.

Aluminum Producers

Reynolds Metal

Research Laboratory

Richmond, VA

R. I. Lindberg

Kaiser Aluminum and Chemical Corporation

Center for Technology

P. O. Box 870

Pleasanton, $\mathrm{CA}$

T. J. Summerson

Alcan Research and Development, Ltd.

P. O. Box 8400

Kingston, Ontario, Canada

T. E. Wright

olin Corporation

91 Shelton Ave.

New Haven, CN

Dr. J. M. Popplewell 

APPENDIX II

CATALOGED DATA 


\section{KEY TO ABBREVIATIONS USED IN TABLE I}

Column Heading

Ident. Number Prod.

Spec. Code

Type of

Specimen

Specimen Size

Depth-Ft.

Type of

Attack

Depth of

Attack
Explanation of Abbreviation

Ident. = Identification

Prod. = Product; Extr. = Extrusion

Spec. = Specimen; $1=$ not welded; 2 an aluminum alloy welded to the same aluminum alloy; 3 = an aluminum alloy welded to a different aluminum alloy

C-Ring = A type of stressed specimen; Ten. Blank = Tensile Blank;

Preform = Another type of stressed specimen

Dia. = Diameter; $T$ = Thickness

Ft. = Feet; T.W. = Tide Water; SED. = Sediment; A.I. = Alternate Immersion

$P=$ Pitting; $I=$ Intergranular; UNI = Uniform; EXFL = Exfoliation; Locl = Localized;

UP = Undermining Pitting; UP+E = Undermining pitting plus exfoliation;

BLIS = Blistering; $\mathrm{P}+\mathrm{I}=$ Pitting plus intergranular; $\mathrm{I}+\mathrm{P}=$ Intergranular plus $\mathrm{Pitting} ;$

$I=$ Intergranular; $\mathrm{P}+\mathrm{EX}=$ Pitting plus Exfoliation; GEN = General; P+BL= Pitting plus

Blistering

Max. = Maximum; PERF = Perforated; NAA = No Appreciable Attack; INCIP = Incipient;

SEVER = Severe; EXTN - Extensive; MOD. = Moderate; MED = Medium; SHAl = Shallow 
TAELE I

\begin{tabular}{|c|c|c|c|c|c|c|c|c|c|c|c|c|c|c|}
\hline $\begin{array}{l}\text { TOENT, } \\
\text { NUMATH }\end{array}$ & $\begin{array}{l}\text { ALH, NY ANn } \\
\text { TFMPF:H }\end{array}$ & PROD. & $\begin{array}{l}\mathrm{SC} \\
\mathrm{Pn} \\
\mathrm{Fn} \\
\mathrm{CP}\end{array}$ & $\begin{array}{l}\text { TYPE nF } \\
\text { SPFCIMFN }\end{array}$ & $\begin{array}{l}\text { SDFCIMFN } \\
\text { SIZE } \\
\text { TNCHES }\end{array}$ & DETAILS RF SPECIMEN & $\begin{array}{l}\text { EXNOSED } \\
\text { DEPTHET }\end{array}$ & $\operatorname{mos}$ & LOCA I JON & ESULTS UF EX & XAMINA & ATIUN & INCHES & $\begin{array}{c}\text { GKAYI, } \\
\text { UATA } \\
\text { RATE }\end{array}$ \\
\hline$-\infty-\infty-\infty$ & $-0-0-+-0-0$ & $---=-$ & $\ldots$ & $=-\pi-\pi-0-m=0$ & $0-0 .-0-0.7$ & 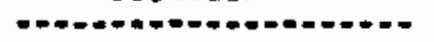 & $-\infty+\infty-\infty+\infty$ & $-\infty$ & 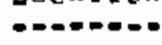 & - & $\rightarrow \infty$ & $\ldots=0$ & - & $-\infty=-\infty$ \\
\hline 38 & $\operatorname{lns} 0-\mathrm{H} 19$ & PI ATR: & 1 & C.RTNC. & $.75 \mathrm{DIA}$ & HLENEME CAL & 5100 & 25 & BASE & ALLOY & & & & \\
\hline 34 & $\ln 60-414$ & PIATE & 1 & C.HNG & .75 DIA & MIAMI FLA & $T w$ & b & BASE & ALLOY & & & & \\
\hline 35 & $\ln 60.414$ & PLATE: & 1 & $C-R T N G$ & .75014 & MIAMI FLA & TOTAL & b & BASE & ALLOY & & & & \\
\hline 96 & $\operatorname{lng} n=414$ & PI ATF & 1 & $C-R I N G$ & .75 DIA & TONGUE OF OCEAN & 5100 & 4 & BASE & ALLGY & & & & \\
\hline 30 & $1+n 0=$ & PIATE & 1 & PBNEL & $9 \times 9 \times .25$ & NAOS ISLAND PACIFIC & 13 & 12 & HASE & ALLOY & $\mathbf{P}$ & & .0060 & .0003 \\
\hline 40 & $1,00=$ & PIATE & 1 & PANTL & $9 \times 9 \times .25$ & NAOS ISLAND PACIFIC & MEANTIDE & 12 & BASE & ALLOY & $P$ & & .010 & .0001 \\
\hline 41 & $\ln 00$ & PI ATE & 1 & PANFL & $9 \times 9 \times .25$ & NAOS ISLAND PACIFIC & 13 & 96 & HASE & ALLOY & $\mathbf{P}$ & & .0110 & .0007 \\
\hline 42 & $\ln n=$ & PI A TF: & 1 & PANFL & $9 \times 3 \times .25$ & NAMS ISLAND PACIFIC & MEANTIDE: & 90 & HASE & ALLUY & $p$ & & .0140 & .0003 \\
\hline 43 & $1100=$ & PLATE & 1 & PANF:L & $9 \times 9 \times .25$ & WATS ISIAND PACIFIC & 13 & 19. & GASE & ALLOY & $\mathbf{P}$ & .033 & .0170 & .0010 \\
\hline 44 & $1: n 0=$ & PIATE. & 1 & PANAL & $9 \times 9 \times .25$ & NADS ISLAND PACIFIC & MEANTIDE & 192 & BASE & ALLOY & $\mathbf{P}$ & .007 & .0390 & .0005 \\
\hline 47 & 190110 & SHEET & 1 & & & TOHGUE OF OCFAN & 5600 & 4 & GABE & ALLOY & $\mathbf{p}$ & PERF & & .0017 \\
\hline 4月 & 19000 & SHEET & 1 & & & TONGIJE DF OCFAN & 5600 & 34 & HASE & ALLUY & $\mathbf{P}$ & PEMF & & .0010 \\
\hline 45 & $1+n 0-F$ & PLATE & 1 & DANEL & $1 \times 2 \times .25$ & TUNGUF OF OCEAN & 4200 & 12 & HASE & ALLUY & $p$ & $.0 \$ 0$ & .030 & \\
\hline 46 & $1100=5$ & PIATF & 1 & PANFI & $1 \times 2 \times .25$ & TUNGUE OF ICFAN & 4200 & 12 & AASE & AILOY & $\mathbf{P}$ & .020 & .020 & \\
\hline a1 & 1,0000414 & PI ATE: & 1 & PANFL & $A \times B \times, 25$ & BKIXHAM ENGLAND & TOTAL & b & HASE & $A L I_{+} \mathbf{O Y}$ & $\mathbf{P}$ & .0000 & & \\
\hline 62 & $1100-\mathrm{H}^{14}$ & PLATE & 1 & PANFL & $8 \times 8 \times, 25$ & ARIXHAN ENGLANO & THTAL & 31 & HASF & ALLOY & $\mathbf{p}$ & .0040 & & \\
\hline$\times 3$ & $1 \mathrm{I} \cap \mathrm{O}-\mathrm{H} 14$ & PIATE & 1 & PANGL & $8 \times 8 \times .25$ & BRIXHAN ENGLAND & TOTAL & bo & HASE & ALLUY & p & .0130 & & \\
\hline 64 & $1 \ln 0-1414$ & PIATH. & 1 & PANFI, & $9 \times R \times, 25$ & BRIXHAM ENGLAND & TOFAL & 120 & BASE & ALLUY & $\mathbf{P}$ & .0110 & & \\
\hline 57 & $1900-H 14$ & PIATF & 1 & DANEL & $12 \times, 2 x .25$ & ESOUIHALT BC & TUTAL & 12 & HASE & $A L I O X$ & $\mathbf{P}$ & .030 & & \\
\hline 59 & $1100=414$ & PT. ATE & 1 & PANFT & $12 \times 12 \times, 25$ & ES'JUTMAI.T RC & TUTAL & 24 & BASE: & AL LOY & $\mathbf{P}$ & .026 & & \\
\hline 59 & $1 \operatorname{1n} 11=14$ & PI ATE. & 1 & DNNET. & $12 \times 12 \times, 25$ & ÉSOJISMALI BC & TUIAL & 60 & BASt. & ALLOY & $\mathbf{P}$ & .015 & & \\
\hline
\end{tabular}


TABLE I

\begin{tabular}{|c|c|c|c|c|}
\hline $\begin{array}{l}\text { IDEHT, } \\
\text { NUHBF: }\end{array}$ & $\begin{array}{l}\text { ALLOY ANn } \\
\text { TRMPER }\end{array}$ & PFOD, & $\begin{array}{l}\text { SC } \\
\text { PO } \\
\text { ED } \\
\text { CE }\end{array}$ & $\begin{array}{l}\text { TYPE } \\
\text { gPPCI }\end{array}$ \\
\hline$=-\infty$ & $-\infty-0+-0+=0$ & $-\infty-\infty$ & $-\infty$ & \\
\hline 60 & $1100-H 14$ & PLATE & 1 & PANFL \\
\hline 53 & $1900=\mathrm{H} 14$ & PLATE & 1 & $P_{\mathbf{R}} \mathrm{H}_{\mathrm{E}} \mathrm{L}$ \\
\hline 54 & $1100-H 14$ & PLATE & 1 & PANEL \\
\hline 55 & $1100-514$ & PLATE & 4 & DANFL \\
\hline 56 & $19000 \mathrm{H} 14$ & PLATE & 1 & $P_{A} N_{E} L$ \\
\hline 49 & $1,00-H_{14}$ & PLATE & 1 & PANFL \\
\hline 50 & $1100-H 14$ & PLATE & 1 & DAMEL \\
\hline 51. & $1100-414$ & PLATE & 1 & PAHFL \\
\hline 52 & $1100 * H 14$ & PLATE & 1 & PANEL \\
\hline 1 & $1900 \cdot \mathrm{H} 14$ & SHEET & 1 & \\
\hline 2 & $1100-H 14$ & SHEET & 1 & \\
\hline 10 & $1100-414$ & SHEET & 1 & \\
\hline 11 & $1100-H_{14}$ & SHERT & 1 & \\
\hline 19 & $1400-\mathrm{H} 14$ & SHEET & 1 & \\
\hline 19 & $11000 \mathrm{H} 14$ & SHEET & 1 & \\
\hline 3 & $1100-414$ & SHEET & 1 & \\
\hline 4 & $19000 H_{14}$ & SHEET & 1 & \\
\hline 12 & $1100=414$ & sheEt & 1 & \\
\hline 13 & $1,00-414$ & SHEET & 1 & \\
\hline 14 & $\ln n g=+14$ & SHFET & 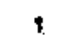 & \\
\hline$\$ 5$ & $1100-H_{14}$ & SHEET & $\mathrm{t}$ & \\
\hline
\end{tabular}

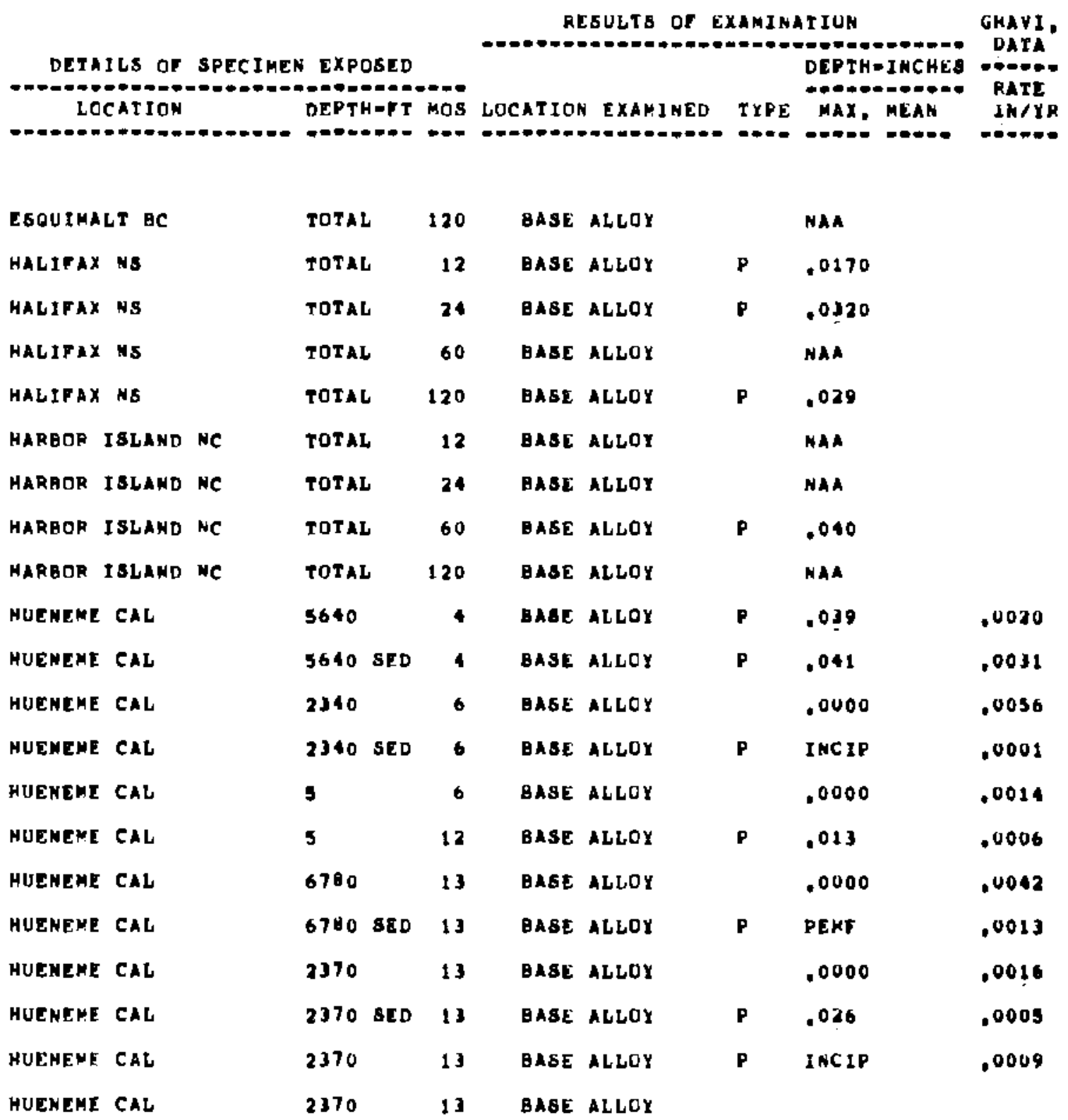


TABLE I

IDENT. ALRT ANT NUMATF

TF.MPER - -

\begin{tabular}{|c|c|}
\hline 16 & $1100-\mu 14$ \\
\hline 17 & $1100=+14$ \\
\hline 5 & $1100=414$ \\
\hline$b$ & $1100=414$ \\
\hline B & $1 \mathrm{~A} \cap \mathrm{O}-\mathrm{H} 14$ \\
\hline 9 & $1100-H 14$ \\
\hline 7 & $1+n 0=n$ \\
\hline 77 & $1160-1014$ \\
\hline 31 & $1960-1414$ \\
\hline 32 & $1160=H 14$ \\
\hline 33 & $1960-H^{14}$ \\
\hline 22 & $1189=$ \\
\hline 73 & 19800 \\
\hline 24 & $1190=$ \\
\hline $2 n$ & $1990-H 14$ \\
\hline$? 1$ & $1980-414$ \\
\hline 25 & 1,090 \\
\hline 2.8 & $1199 \circ$ \\
\hline 26 & $1199=$ \\
\hline 79 & $1109=$ \\
\hline
\end{tabular}

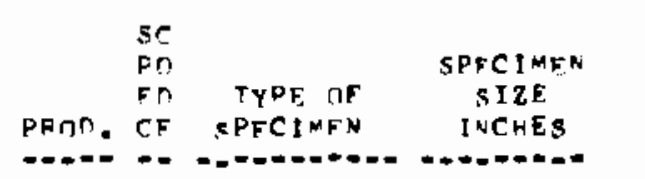

\begin{tabular}{|c|c|c|}
\hline SHFFT & 1 & $.051 \mathrm{~T}$ \\
\hline SHEFT & 1 & $.051 \mathrm{~T}$ \\
\hline SHEET & 1 & .051 T \\
\hline SHEFI & 1 & .0511 \\
\hline SHEET & $:$ & $.051 \mathrm{~T}$ \\
\hline \multirow[t]{2}{*}{ SHEET } & 9 & $.051 \mathrm{~T}$ \\
\hline & 1 & \\
\hline SHEFT & I DANGL & $3 \times 9 \times .064$ \\
\hline SHEET & ! D.tith & $3 \times 3 \times .004$ \\
\hline SHEFT & $\Rightarrow D_{n} N_{F l}$ & $3 \times 9 \times, 064$ \\
\hline \multirow[t]{4}{*}{ SHEFT } & $1 D_{A} D_{F}$ & $3 \times 9 \times .064$ \\
\hline & 1 & \\
\hline & 1 & \\
\hline & $t$ & \\
\hline SHEFT & 1 & .0517 \\
\hline SHEFT & 1 & .0511 \\
\hline SHEFT & 1 Dotict & $.05 \times 4 \times 12$ \\
\hline SHEET & 1 DNEI: & $.05 \times 4 \times 12$ \\
\hline SHETt & $1 D_{A} \times 2$ & $.15 \times 4 \times 17$ \\
\hline SHEET & 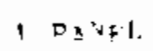 & $.75 \times 4 \times 12$ \\
\hline
\end{tabular}

RESULTS OF EXAMINATIUM

\begin{tabular}{|c|c|c|c|}
\hline & INCLP & & .0020 \\
\hline & $I \times C I P$ & & . vout \\
\hline & .0053 & .0000 & .0002 \\
\hline & .0117 & .0024 & .0002 \\
\hline & .0036 & .0010 & .0001 \\
\hline & .0065 & $.00<5$ & \\
\hline
\end{tabular}


TAELE I

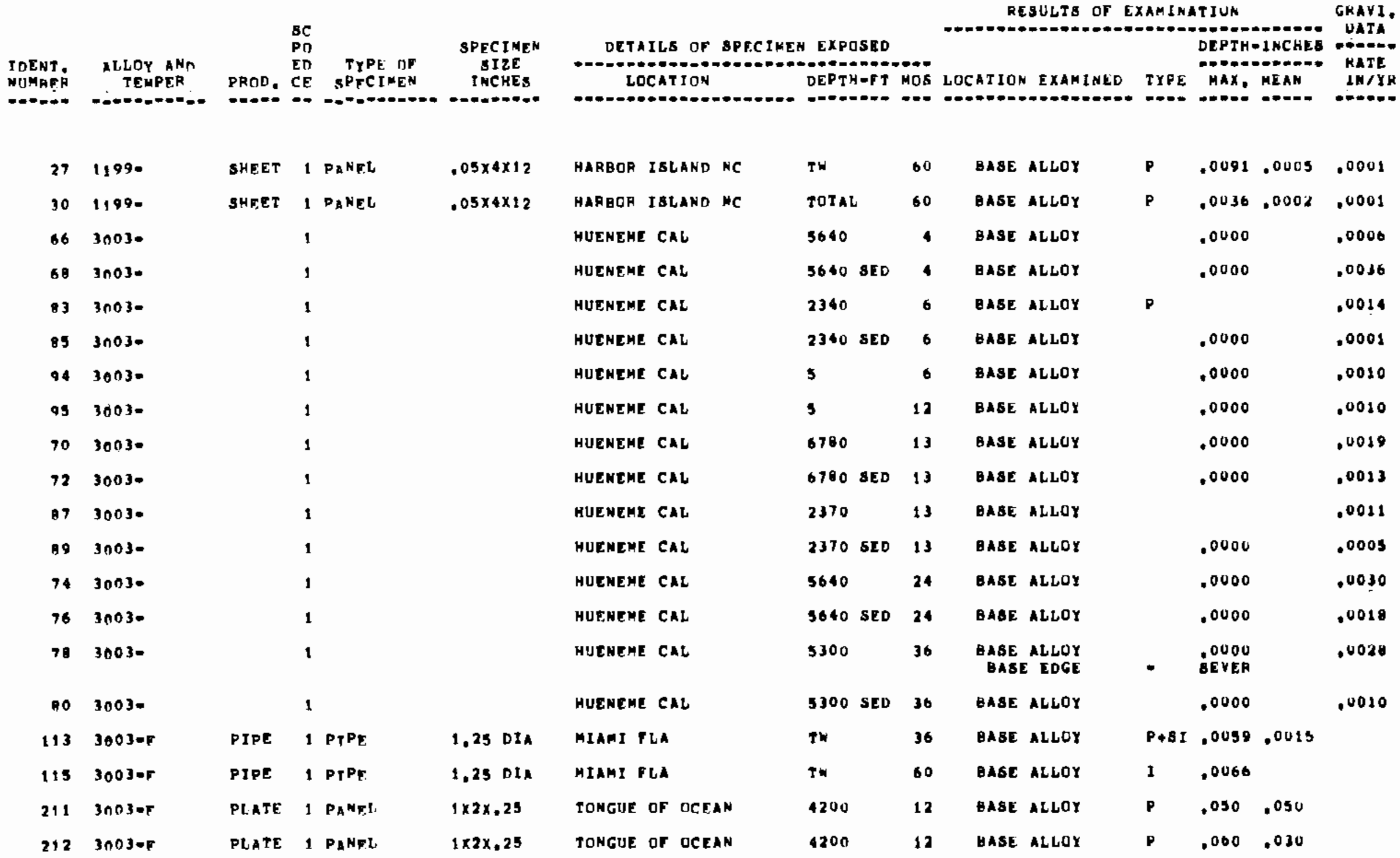


TABLE I

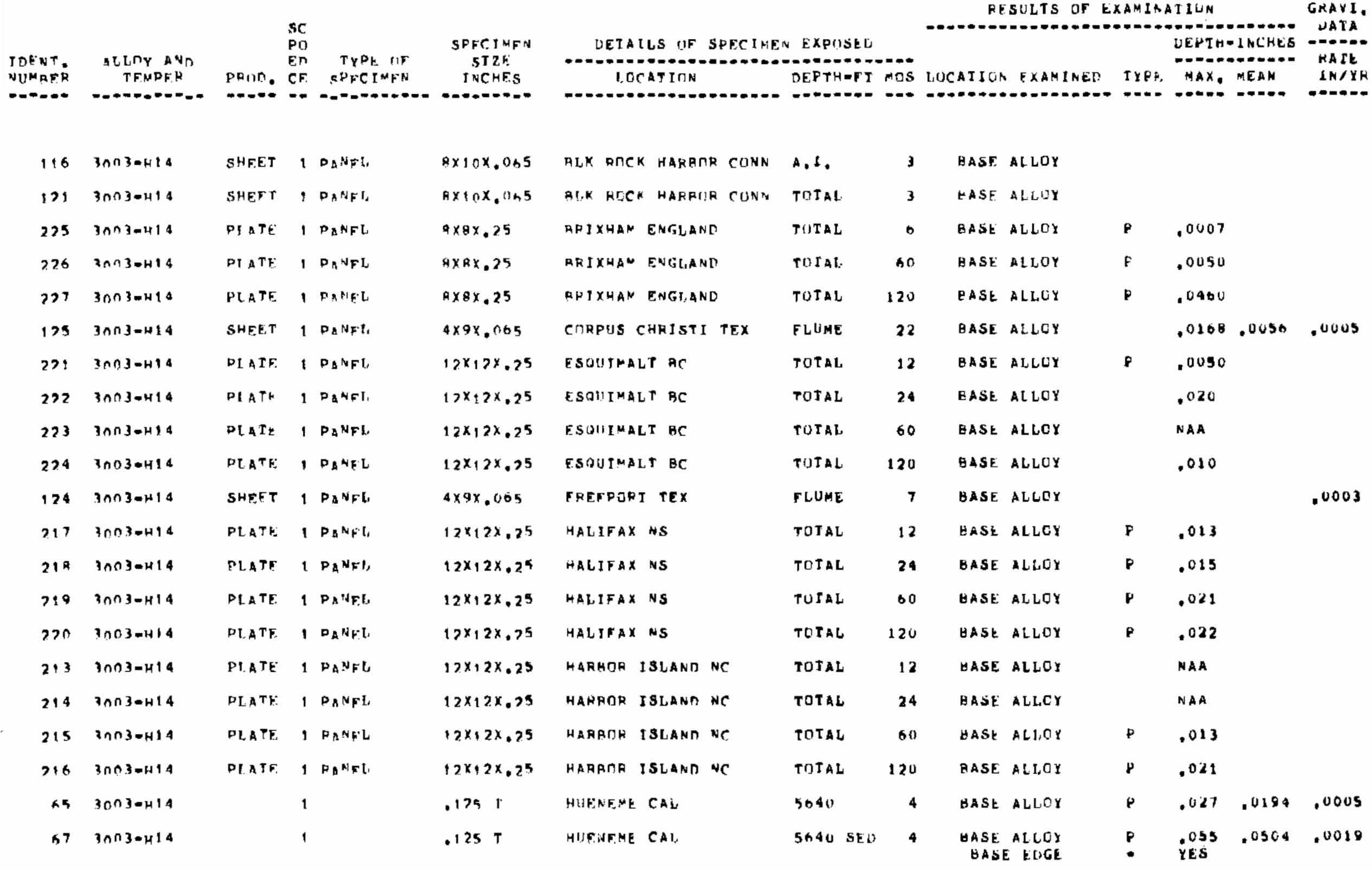


TABLE I

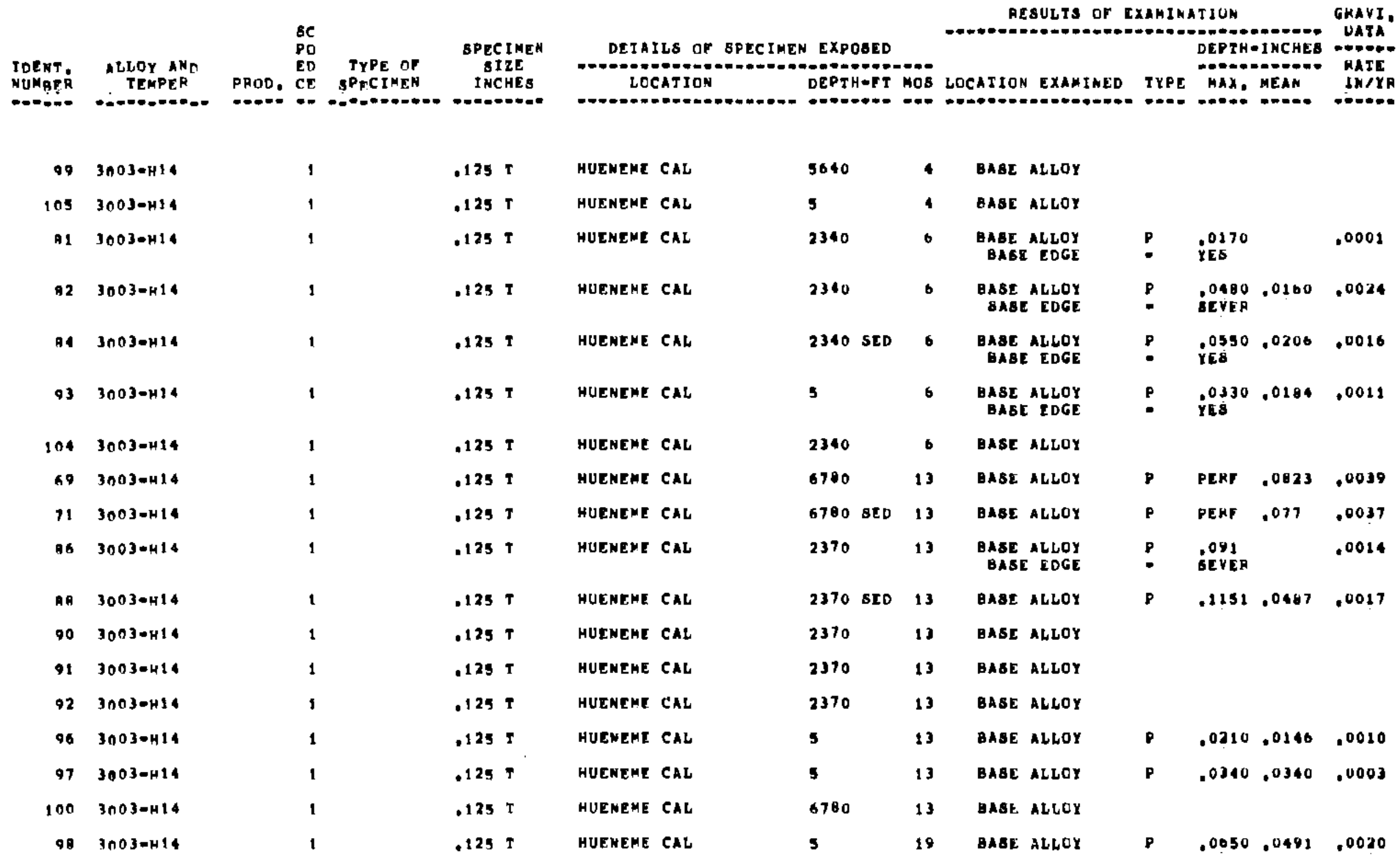


TAGLE I

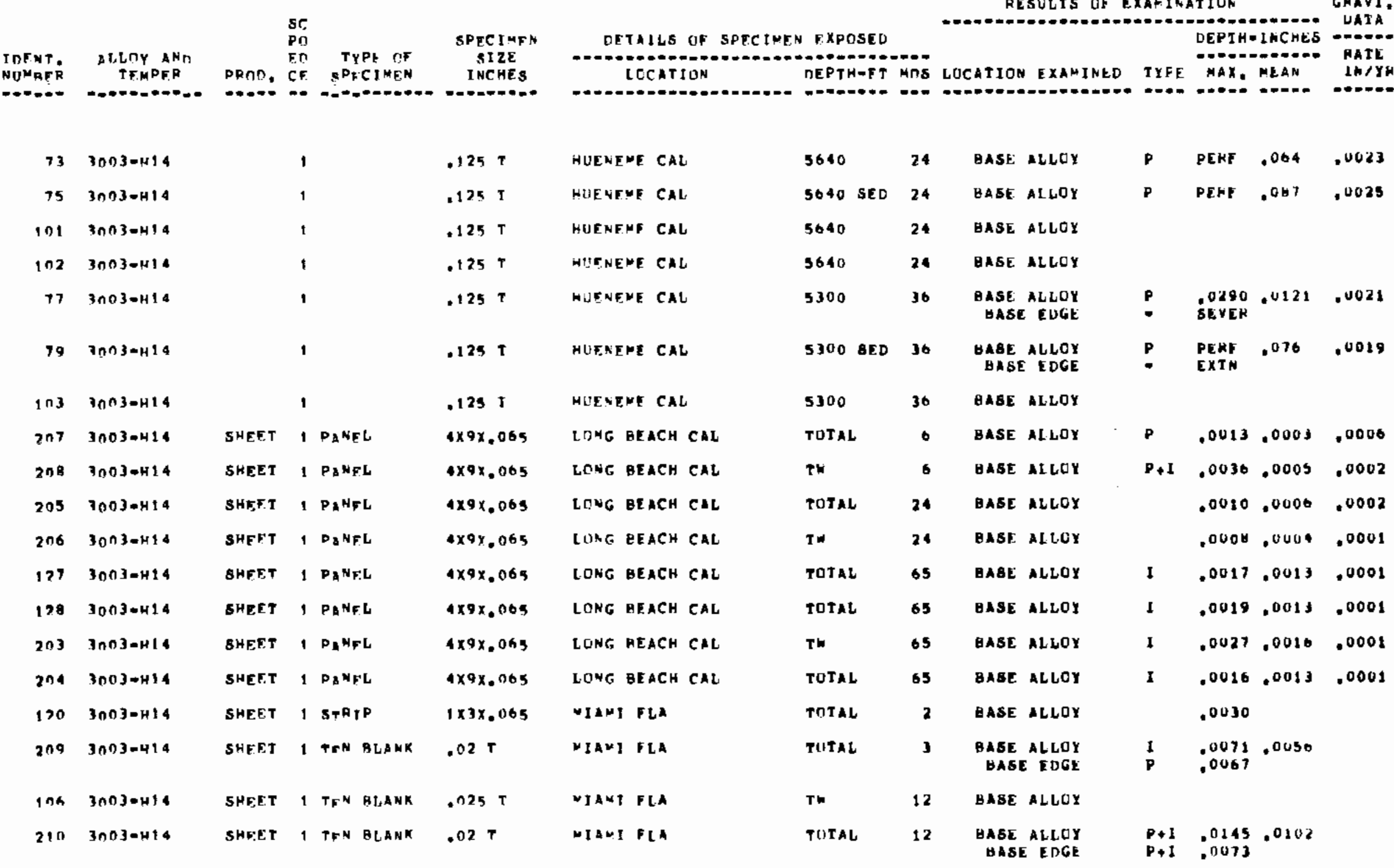


TABLE I

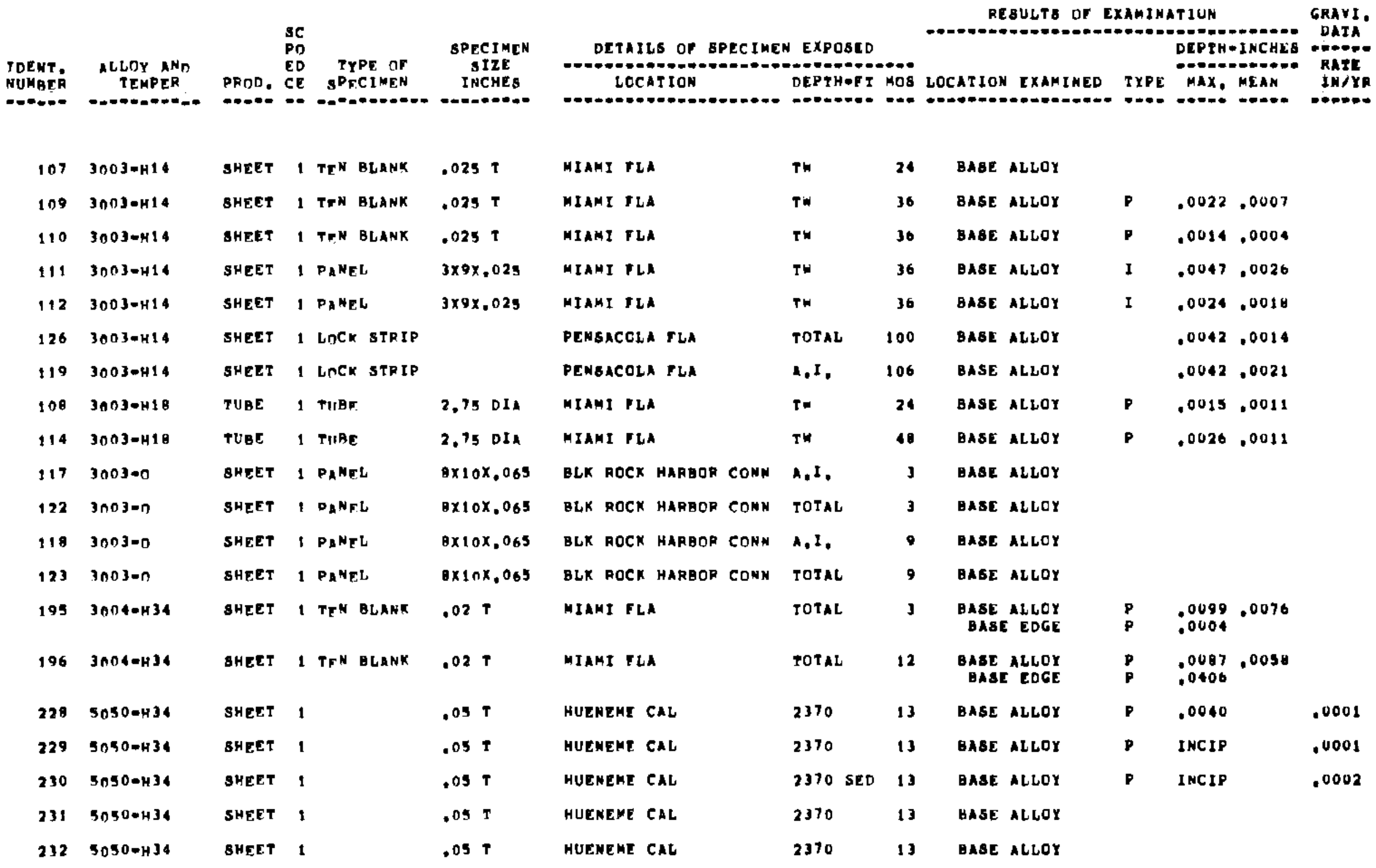


TABLE I

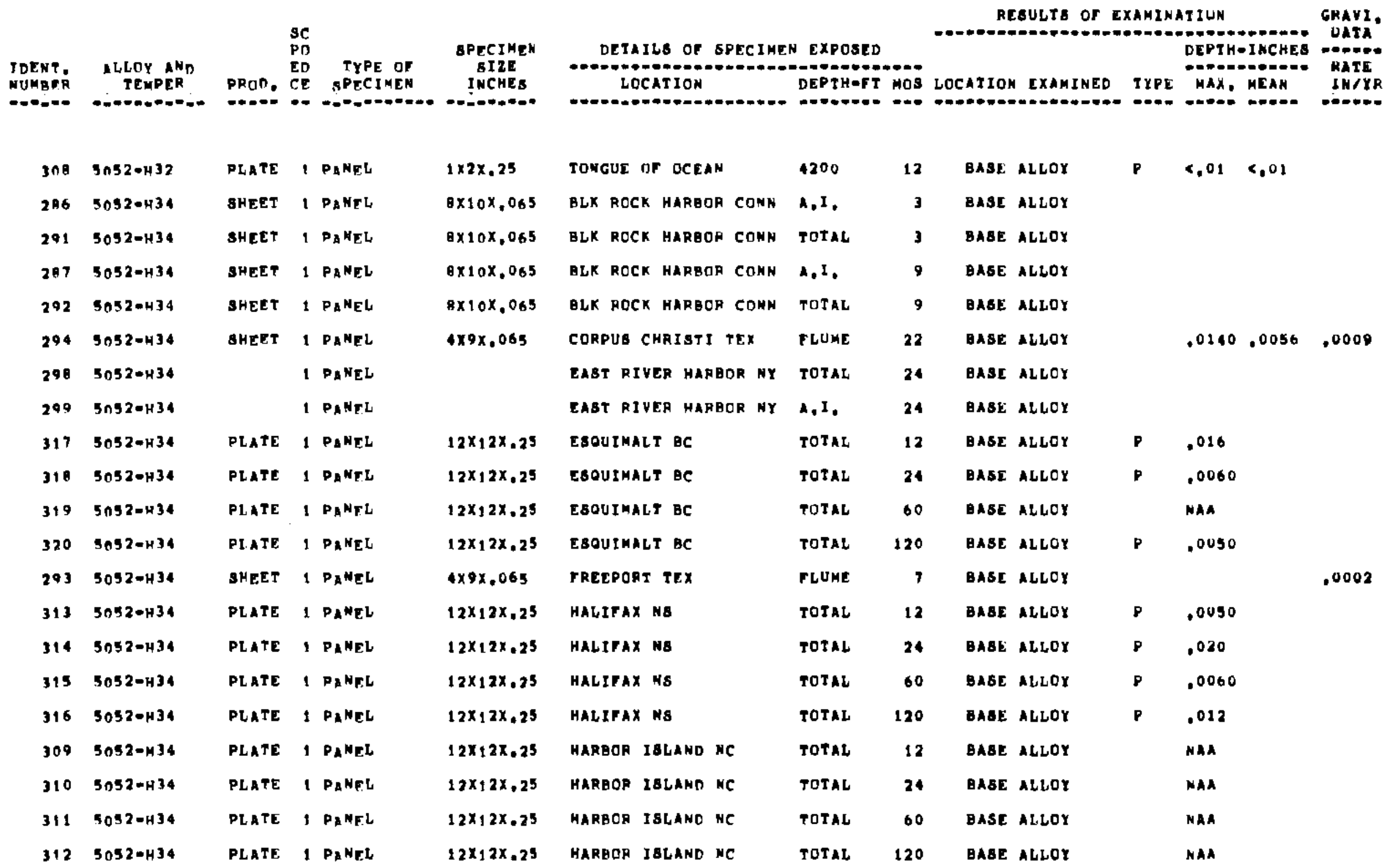


TABLE I

\begin{tabular}{|c|c|c|c|c|c|c|c|c|c|c|c|c|c|c|}
\hline 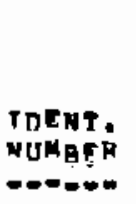 & $\begin{array}{l}\text { MLNY ANn } \\
\text { TEMPER } \\
\text { TEMER }\end{array}$ & PARD. & 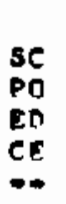 & $\begin{array}{c}\text { TYPE OF } \\
\text { SPFCIMEN } \\
-\end{array}$ & $\begin{array}{l}\text { SPECIHEN } \\
\text { BIZE } \\
\text { INCHES } \\
\end{array}$ & $\begin{array}{l}\text { DETAILS or SPECTMEN } \\
\text { LOCATION }\end{array}$ & 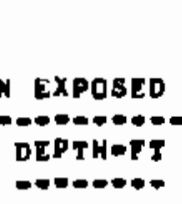 & MOS & LOCATIOA & N EXAHINED & TXPE & 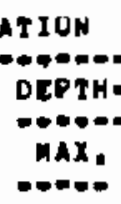 & IMCHES & $\begin{array}{c}\text { GKAYI, } \\
\text { DATA } \\
\text { RATE } \\
\text { RAMYR } \\
\text { LNY }\end{array}$ \\
\hline 290 & $5 \times 520 H^{34}$ & BHEET & 1 & TFN BLANK & $.025 \mathrm{~T}$ & MIAMI TLA & It & 36 & GABE & ALLOY & $\nabla$ & .0010 & .0004 & \\
\hline 281 & $5052 \cdot 1434$ & SHERT & 1 & TFN BLANK & $.025 \mathrm{~T}$ & MIAMI TLA & TW & 36 & BABE & ALLOY & $\nabla$ & .0009 & .0003 & \\
\hline 282 & $5052=134$ & SHEET & 1 & $P_{A}$ NEL & $3 \times 9 \times .025$ & MIANI TLA & It & 36 & SASE & ALLOY & $\mathbf{p}$ & .0023 & .0016 & \\
\hline 293 & $5052-H 34$ & SHEET & 1 & PANEL & $3 \times 9 \times .025$ & MIAMI VLA & $T w$ & 36 & BABE & ALLOX & $\nabla$ & .0020 & .0013 & \\
\hline 284 & $5052-434$ & SHEET & 1 & PANEL & $9 \times 9 \times, 065$ & MIAMI TLA & Tr & 48 & BASE & ALLOY & & & & \\
\hline 302 & $5052-\mathrm{H}^{34}$ & & 1 & PaNEL & & MDBILE ALA & TOTAL & 24 & GABE & ALLOY & & & & \\
\hline 303 & $5052=434$ & & 1 & PQNTL & & MOBILE ALA & A,I. & 24 & BABE & ALLOX & & & & \\
\hline 296 & $5052-434$ & & 1 & PANFL & & MONTREAL OUE & TOTAL & 12 & BABE & ALLOY & & & & \\
\hline 207 & $5052-\mathrm{H}_{3} 4$ & & 1 & PANEL & & MONTREAL OUE & A,I, & 12 & onsE & ALLDY & & & & \\
\hline $3 n 4$ & $5052-H^{34}$ & & 1 & PANEL & & MEH OALEANS LA & TOTAL & 24 & BASE & ALLOY & & & & \\
\hline 305 & $5052-\mathrm{H}_{34}$ & & 1 & PANEL & & NEN ORLEANS LA & A, I. & 24 & GABE & ALLOY & & & & \\
\hline 298 & $5052-\mathrm{H} 34$ & SHEET & 1 & LOCK STRIP & $.065 \mathrm{~T}$ & PEMBACOLA TLA & A,I, & 100 & BASE & ALLOY & & .0094 & .0015 & \\
\hline 295 & $5052-\mathrm{H} 34$ & SHEET & 1 & LOCK STRIP & $.065 T$ & PEMSACola rla & TOTAL & 100 & BASE & ALLOY & & .0014 & .0056 & \\
\hline 285 & $5052-n$ & SHEET & 1 & PANEL & $8 \times 10 \times, 065$ & BLK ROCK HARBOR COMN & A, I, & $\mathbf{3}$ & BAse & ALLOI & & & & \\
\hline 290 & $3052-n$ & SHEET & 1 & PANEL & $8 \times 10 \times .065$ & BLK ROCK HARBOA CONH & TOTAL & 3 & BASE & ALLOY & & & & \\
\hline 234 & $\sin 52-0$ & SHEET & 9 & & $.065 \mathrm{~T}$ & HUENEME CAL & 3640 & 4 & BASE & ALLOY & & .0000 & & .0037 \\
\hline 235 & $\operatorname{sos} 2-0$ & SHEET & 1 & & $.065 \mathrm{~T}$ & HUENEME CAL & 5640 ard & 4 & BASE & ALLOY & & .0000 & & .0040 \\
\hline 243 & $\operatorname{sos} 2=0$ & SHEET & 1 & & $.069 \mathrm{~T}$ & hUENEMT CAD & 2340 & 6 & BABE & ALLOY & & .0000 & & .0028 \\
\hline 244 & $5052-n$ & SHEET & 1 & & $.065 \mathrm{~T}$ & HUEMEME CAL & 2340 SED & 6 & BABE & ACLOX & $\mathbf{P}$ & INCIP & & .0001 \\
\hline 247 & $5052=n$ & SHEET & 1 & & $.065 \mathrm{~T}$ & HUENEME CAL & s & 6 & BASE & ALLOX & $\mathbf{P}$ & INGEP & & .0012 \\
\hline 248 & $\sin 20 \pi$ & BHEET & 1 & & $.065 \mathrm{~T}$ & HUENEME CAL & 5 & 12 & BABE & ALLOY & $\mathbf{P}$ & .0050 & & .0000 \\
\hline
\end{tabular}


TABCE I

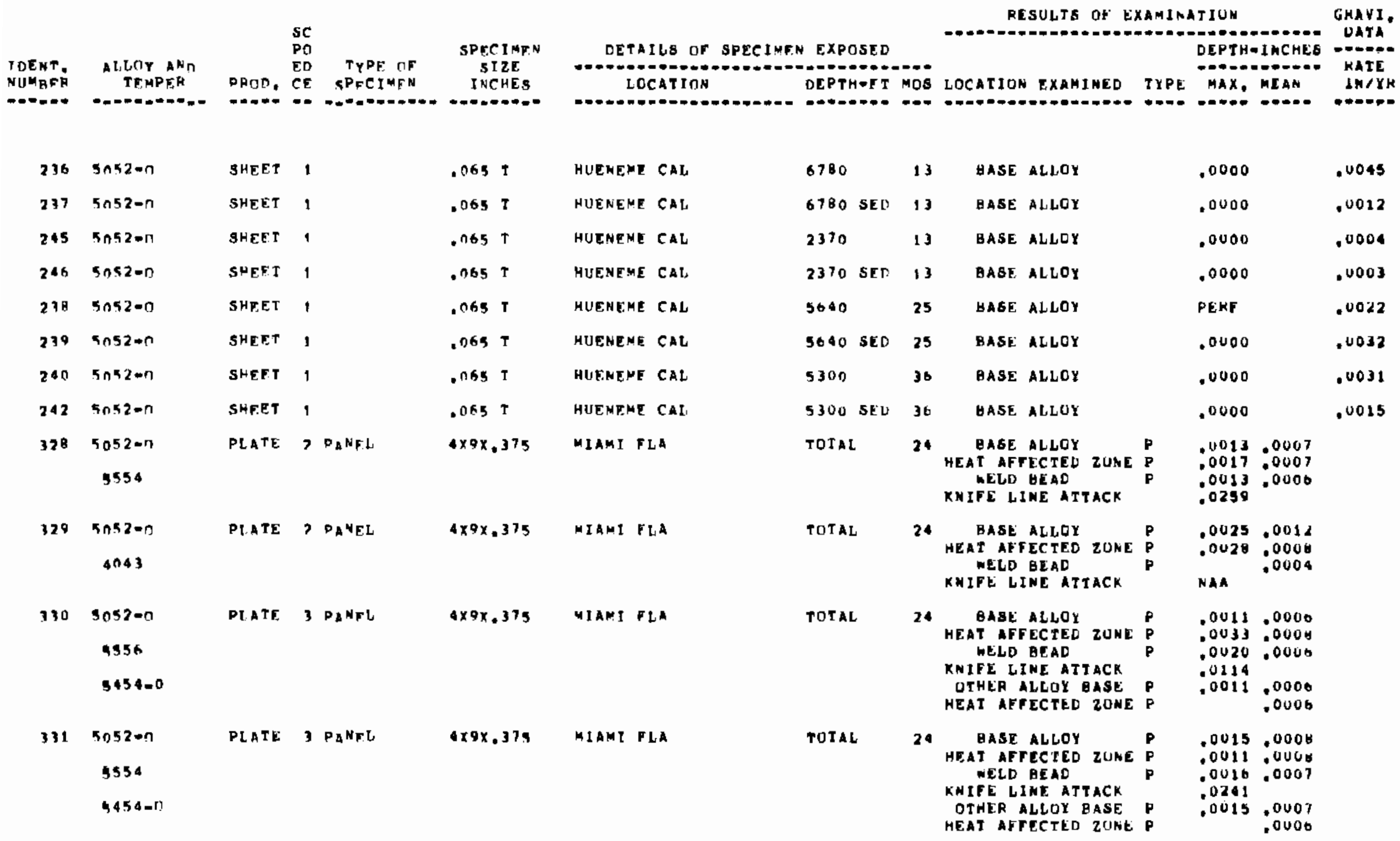


TABLE I

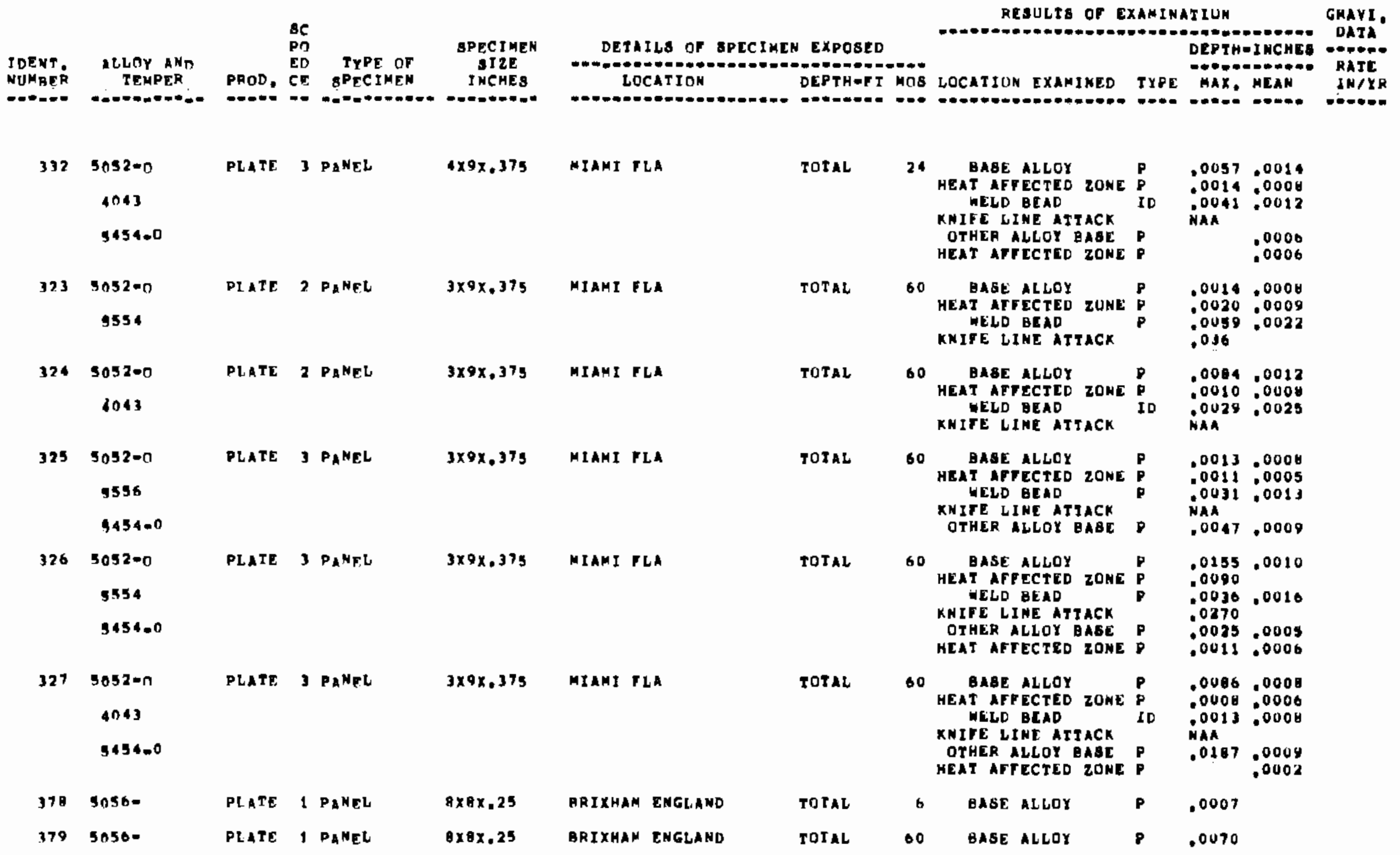


TABLE I

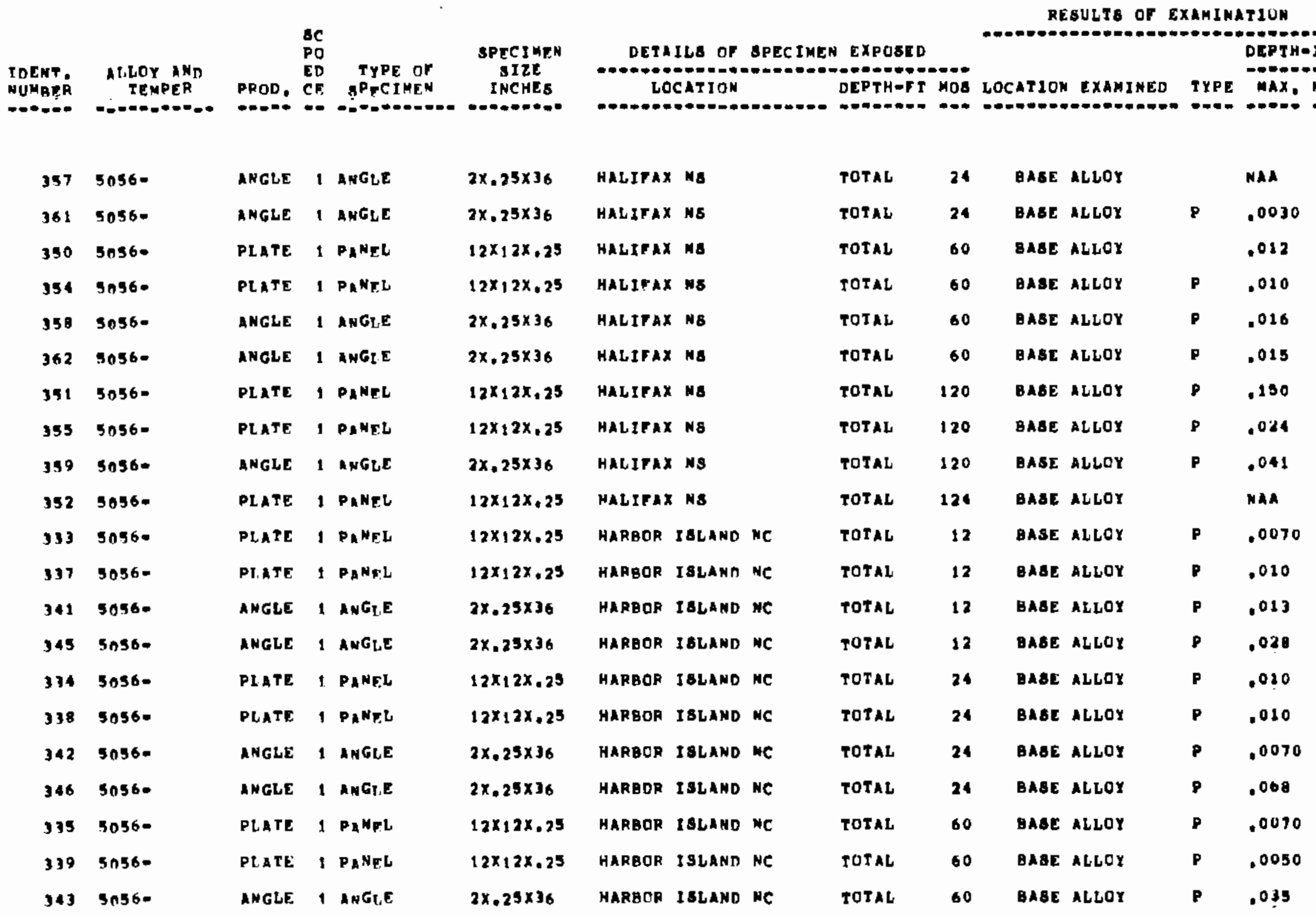


TABLE I

\begin{tabular}{|c|c|c|c|c|c|c|c|c|c|c|c|c|}
\hline $\begin{array}{l}\operatorname{InENT}: \\
\text { NIMRFFP }\end{array}$ & $\begin{array}{l}\text { SLIMY ANn } \\
\text { TR.MPER }\end{array}$ & PROD. & $\begin{array}{l}\text { Sr } \\
\text { PO } \\
\text { ED } \\
\text { CE }\end{array}$ & $\begin{array}{r}\text { TYPE } \cap F \\
\text { SPFCIMFN }\end{array}$ & $\begin{array}{l}\text { SPECIMFN } \\
\text { SIZE. } \\
\text { INCHES }\end{array}$ & $\begin{array}{l}\text { DETAILS OF SPECIMEN } \\
\text { LCCATION }\end{array}$ & $\begin{array}{l}\text { EXPOSED } \\
\text { DEPTH-FI }\end{array}$ & M०ड & LOCATION & N EXAMINED & TYFE & $\begin{array}{l}\text { DEPTHA } \\
\text { MAX. }\end{array}$ \\
\hline$+\infty-\infty$ & $\cdots+-\infty-0-00$ & $\cdots \infty$ & $\cdots$ & n-men & $\cdots$ & 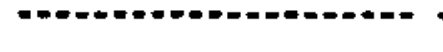 & $+-0.0+0.0$ & $\cdots$ & $m-\infty-\infty$ & (n) & $\cdots \infty$ & $\cdots \infty$ \\
\hline 347 & 50560 & ANGLE & 1 & $A N G t, E$ & $2 \times .25 \times 38$ & HARROE ISLAND NC & TOTAL & 60 & BASE & ALLOY & $\mathbf{P}$ & .017 \\
\hline 336 & $5 n 56=$ & PLATE & 1 & PANFL & $12 \times 12 \times 25$ & HARBOR ISLAND NC & TOTAL & 120 & BASE & ALLOY & $\mathbf{p}$ & .0050 \\
\hline 340 & $5056=$ & DTATE & 1 & PANFL & $12 x_{1} 2 x_{2} 25$ & HAFBOR ISLAND NC & TOTAL & 120 & BASE & ALLOY & $\mathbf{p}$ & .028 \\
\hline 344 & $5056=$ & ANGLE & i & AMGIE & $2 \times .75 \times 36$ & HAREOP ISIJAND NC & TOTAL & 120 & BASE & ALLOY & $\mathbf{P}$ & .032 \\
\hline 410 & $5083=$ & PLATE: & 1 & DANFL & $8 \times 8 \times .25$ & BRIXHAM ENGLAND & TOTAL & b & BASE & ALLOY & $\mathbf{P}$ & .0060 \\
\hline $41 \mathrm{t}$ & $5 \cap 83=$ & PLATE & 1 & $P_{A} N_{F} L$ & $9 \times A \times .25$ & BRIXHAM ENGLAND & TOTAL & so & BASE & ALI.OY & $\mathbf{P}$ & .0030 \\
\hline 412 & $\operatorname{sn} \times 3=$ & PLATE & 1 & PANEL & $8 \times P \times, 25$ & BPIXHAM ENGLAND & TOTAL & 120 & GASE & ALLUY & $p$ & .034 \\
\hline 1114 & $50 \times 3=$ & PT ATE & 1 & PANFL & $12 \times 12 \times .25$ & ESOUIMALT BC & TOTAL & 12 & BASE & ALLOY & $\mathbf{P}$ & .013 \\
\hline 1118 & SnA30 & PIATE & 1 & $\mathrm{PA}_{\mathrm{A}} \mathrm{N}_{\mathrm{T}}$ & $12 x_{1} 2 x_{.25}$ & ESOUIVALT BC & TOTAL & 12 & BASE & ALLOY & $\mathbf{P}$ & .029 \\
\hline 1172 & $5 \cap \times 3=$ & PLATE: & 1 & $P_{A} N_{F} L$ & $12 \times 12 \times .25$ & ESOUIMALT BC & TOTAL & 12 & BASE & ALLOY & & NAA \\
\hline 1115 & $5083=$ & PLATE & 1 & PANEL & $12 x_{1} 2 x_{.25}$ & ESUUIMALT BC & TOTAL & 24 & BASE & ALLCY & $\mathbf{P}$ & .0050 \\
\hline 1119 & $5083=$ & PLATE & 1 & PANFL & $12 x_{1} 2 x_{2} 5$ & ESOHIMAT BC & TUTAL & 24 & BASE & ALLOY & $\mathbf{P}$ & .038 \\
\hline 1123 & $5083=$ & PLATE & 1 & PANEL & $12 \times 12 x=25$ & ESOUTMALT BC & TOTAL & 24 & BASE & ALLOX & & NAA \\
\hline 1116 & SOA3- & PLATF & 1 & PANFL & $12 x_{1} 2 x_{2} 5$ & ESOUIMALT BC & TOTAL & 60 & BASE & ALLOY & & NAA \\
\hline 1120 & $50 \times 3=$ & PLATE & 1 & PANEI & $12 \times+2 \times, 25$ & ESOUMALT BC & TOTAL & 60 & BASE & ALLOY & $\mathbf{P}$ & .047 \\
\hline 1124 & $5083-$ & PLATE & 1 & PANEL & $12 \times 12 \times, 25$ & ESOUIMALT BC & TOTAL & 60 & EASE & ALLOY & $\mathbf{P}$ & .0050 \\
\hline 1117 & $5083=$ & PLATF. & 1 & PANFL & $12 \times 12 x, 25$ & ESOUIMALT BC & TOTAL & 120 & BASE & ALLOY & $P$ & .0060 \\
\hline 1121 & $3083=$ & PLATE & 1 & PANEL & $12 x_{1} 2 \times, 25$ & ESOIIAALT BC & TOTAL & 120 & BASE & ALLOY & $P$ & .055 \\
\hline 1103 & $50 \times 3=$ & PT.ATE & 1 & $P_{A} N_{F} I_{1}$ & $12 x_{1} 2 x_{0} 25$ & HALIFAX NS & TOTAL & 12 & BASE & ALLOY & $P$ & .0030 \\
\hline 1107 & $5083=$ & PI ATE & 1 & PANFL & $12 x_{1} 2 \times .25$ & HALIFAX NS & TOTAL & 12 & HASE & ALLOX & $P$ & .0040 \\
\hline $1+11$ & $5 \cap 83=$ & PTATE & 1 & $P_{A} N_{F} L$ & $12 x_{1} 2 x, 25$ & HALIFAX NS & TOTAL & 12 & HASE & ALLOX & & NAA \\
\hline
\end{tabular}


TABLE I

\begin{tabular}{|c|c|c|c|c|}
\hline $\begin{array}{l}\text { TOENT. } \\
\text { NUMBER }\end{array}$ & $\begin{array}{l}\text { ALLOY AND } \\
\text { TEMPER }\end{array}$ & PRDD. & $\begin{array}{l}\text { 6C } \\
\text { PO } \\
\text { ED } \\
\text { CE. }\end{array}$ & $\begin{array}{l}\text { TYP } \\
\text { P FC }\end{array}$ \\
\hline$-\theta-\infty-\infty$ & $+\infty+\infty+0,0=0$ & $-\infty=\infty$ & $=$ & -0 \\
\hline 1104 & $5003=$ & PLATE & 1 & PANTL \\
\hline 1108 & 5กค 3- & PLATE & 1 & PANEL \\
\hline 1112 & 50930 & PLate & 1 & PANEL \\
\hline 1105 & $5083=$ & PLATE & 1 & PaNEL \\
\hline 1409 & 50830 & PLATE & 1 & PAHEL \\
\hline 1113 & $5083=$ & PLATE & 1 & PANEL \\
\hline 1106 & 50830 & PLATE & 1 & PANEL \\
\hline 1110 & $5083=$ & PLATE & 1 & PENTLL \\
\hline 475 & $5083=$ & PLate. & 1 & PANEL \\
\hline 1096 & $50 \times 3 \cdot$ & Plate & 1 & PANEL \\
\hline 1100 & $5083=$ & PLATE & 1 & PANEL \\
\hline 476 & $5083=$ & Plate & 1 & PANEL \\
\hline 1097 & $5083=$ & plate & 1 & PANEL \\
\hline 1101 & $5003=$ & PLate & 1 & PANEL \\
\hline 1094 & 50830 & PLATE & 1 & PANEL \\
\hline 1098 & $5003=$ & PLATE & 1 & PANEL \\
\hline 1102 & 50030 & PLATE & 1 & PANEL \\
\hline 1095 & $5003 \cdot$ & PLATE & 1 & PANEL \\
\hline 1099 & 50030 & PLATE & 1 & PanTL \\
\hline 435 & $5003=$ & & 1 & \\
\hline 437 & 50830 & & 1 & \\
\hline
\end{tabular}

\section{DETAILS OF APECIMEN EXPOBED}

SPEC IMEN

SIZE I HCHES

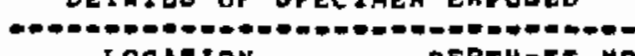
LOCATIOH DEPTH-TT MOS

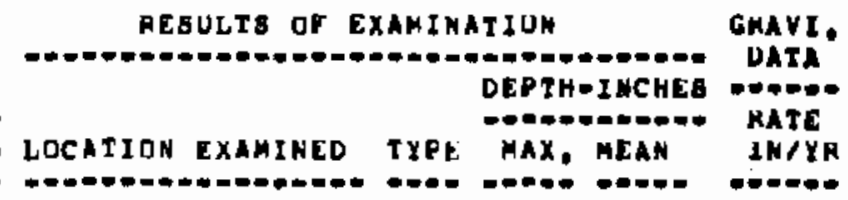

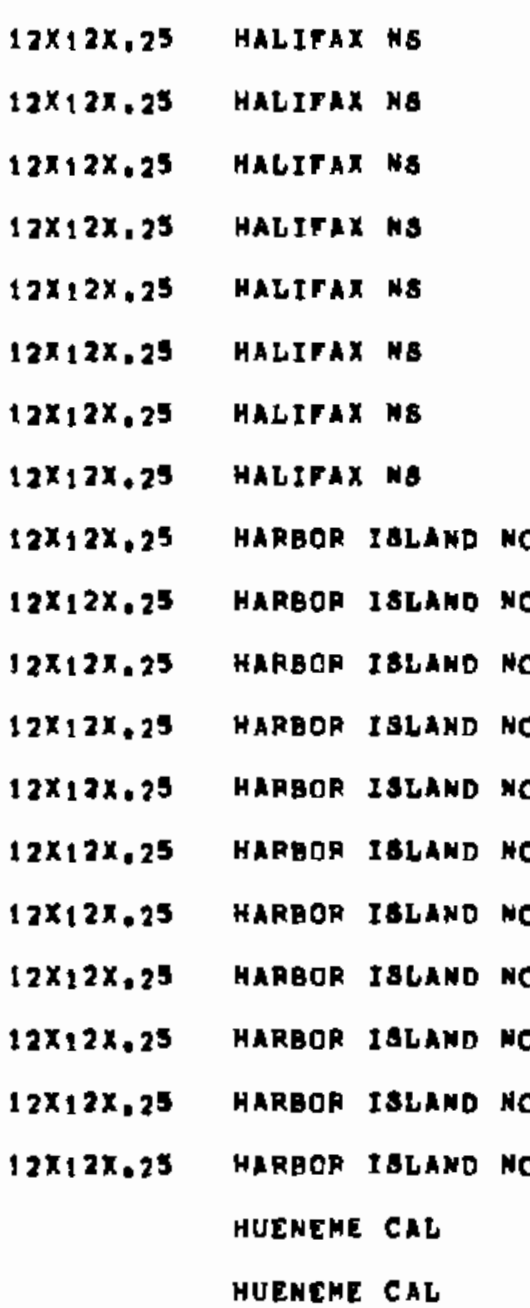

\begin{tabular}{|c|c|c|c|c|c|}
\hline TOTAL & 24 & BASE & ALLOY & & NAA \\
\hline TOTAL & 24 & BASE & ALLOY & $P$ & .023 \\
\hline Total & 24 & BASE & ALLOY & $\mathbf{P}$ & .021 \\
\hline TOTAL & 60 & BABE & ALLOY & $P$ & .012 \\
\hline TOTAL & 60 & BASE & ALLOY & $\mathbf{P}$ & .016 \\
\hline TOTAL & 60 & DASE & ALLOY & $\mathbf{P}$ & .0070 \\
\hline TOTAL & 120 & BASE & ALLOY & $\mathbf{p}$ & .0070 \\
\hline POTAL & 120 & BABE & ALLOY & $P$ & .022 \\
\hline TOTAL & 12 & GABE & ALLOY & $\mathbf{P}$ & .012 \\
\hline TOtAL & 12 & Bast & ALLOY & $\mathbf{P}$ & .016 \\
\hline TOTAL & 12 & BASE & ALLOY & $\mathbf{P}$ & .022 \\
\hline TOTAL & 24 & BABE & ALLOY & $\mathbf{p}$ & .0090 \\
\hline Total & 24 & BASE & ALLOY & $\mathbf{P}$ & .023 \\
\hline TOTAL & 24 & HABE & ALLOY & $\mathbf{P}$ & $.00 \pm 0$ \\
\hline TOTAL & 60 & BABE & ALLOY & $P$ & .0060 \\
\hline TOTAL & 60 & BASE & ALLOY & $\mathbf{P}$ & .0060 \\
\hline rotAL & 60 & BABE & ALCOY & $\mathbf{p}$ & .0070 \\
\hline TOTAL & 120 & BA8E & ALLOY & & WAA \\
\hline TOTAL & $\$ 20$ & BASE & ALLOY & P & .010 \\
\hline 5 & 0 & BABE & ALLOY & ETCH & \\
\hline 5 & 12 & BABE & ALLOY & ETCH & \\
\hline
\end{tabular}


TAEIE I

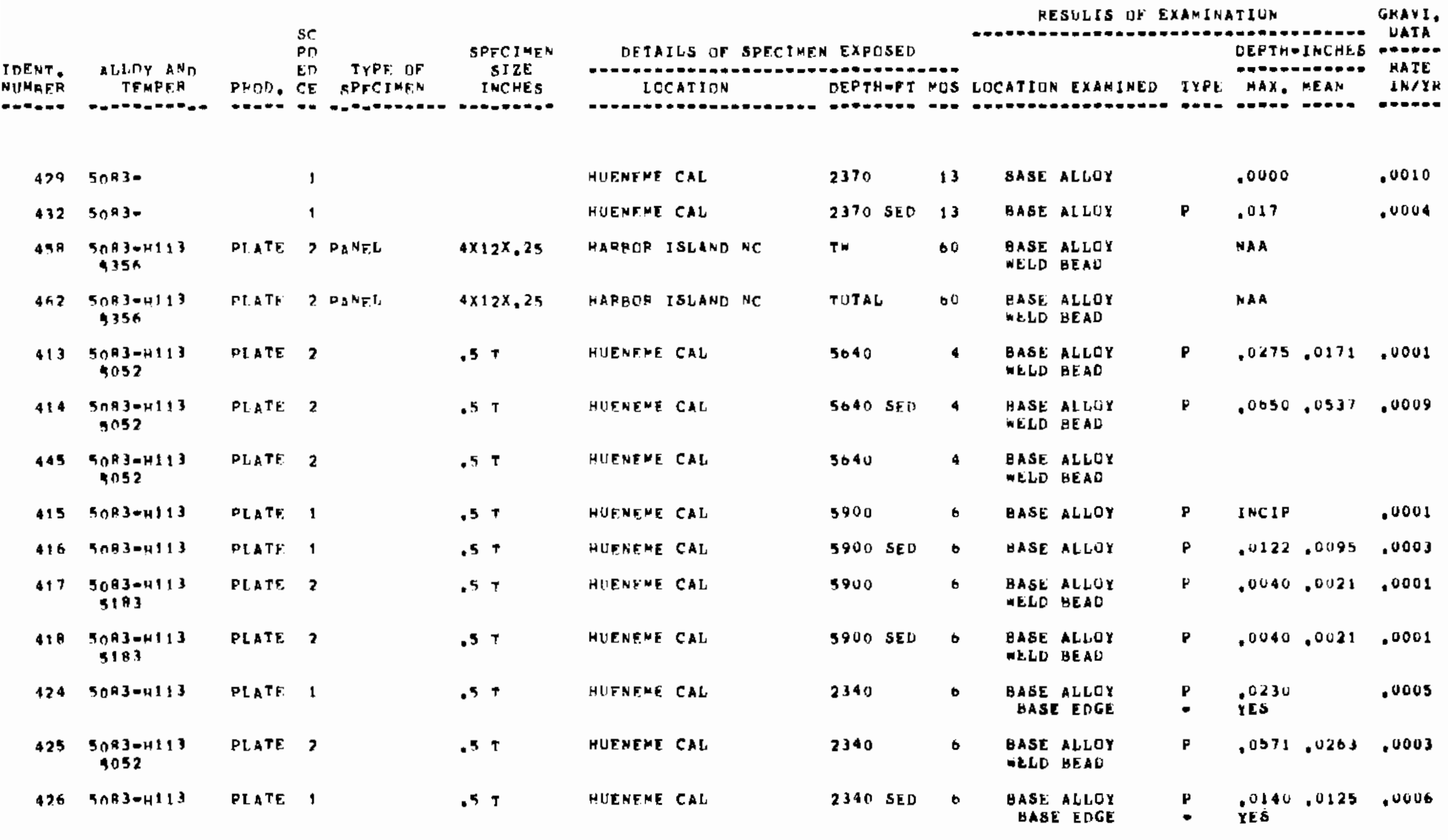


TABLE I

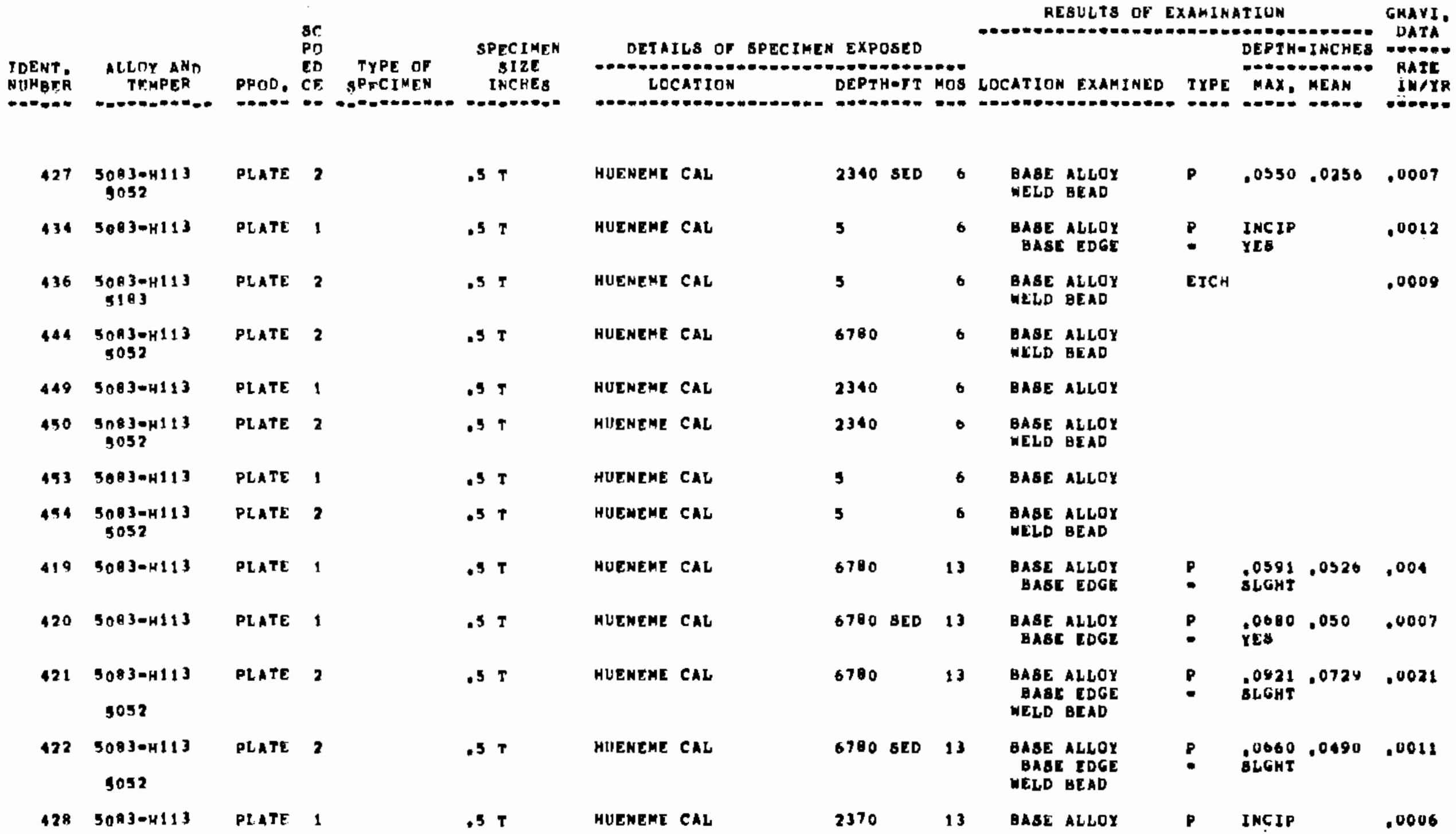


TABLE I

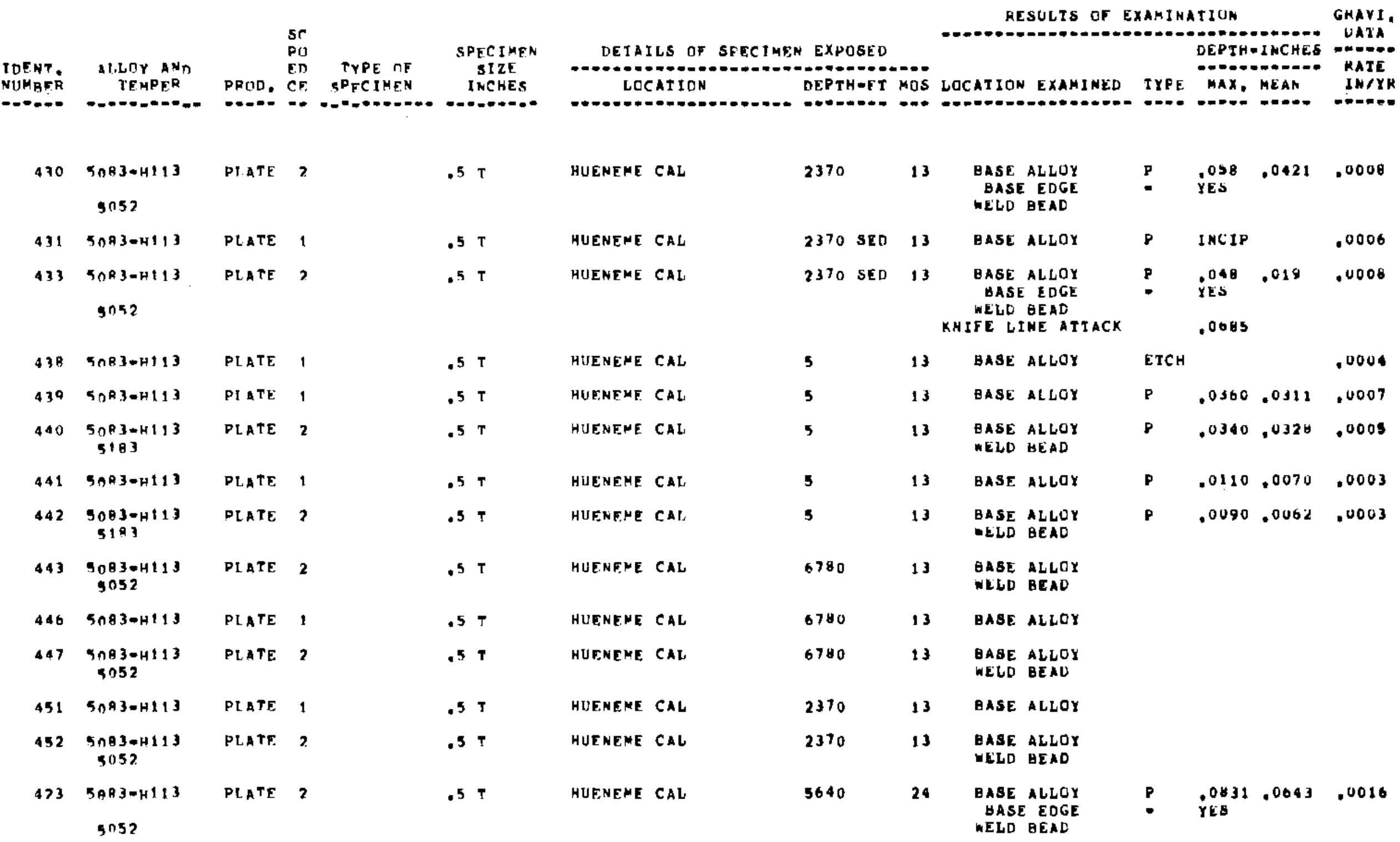


TABLE I

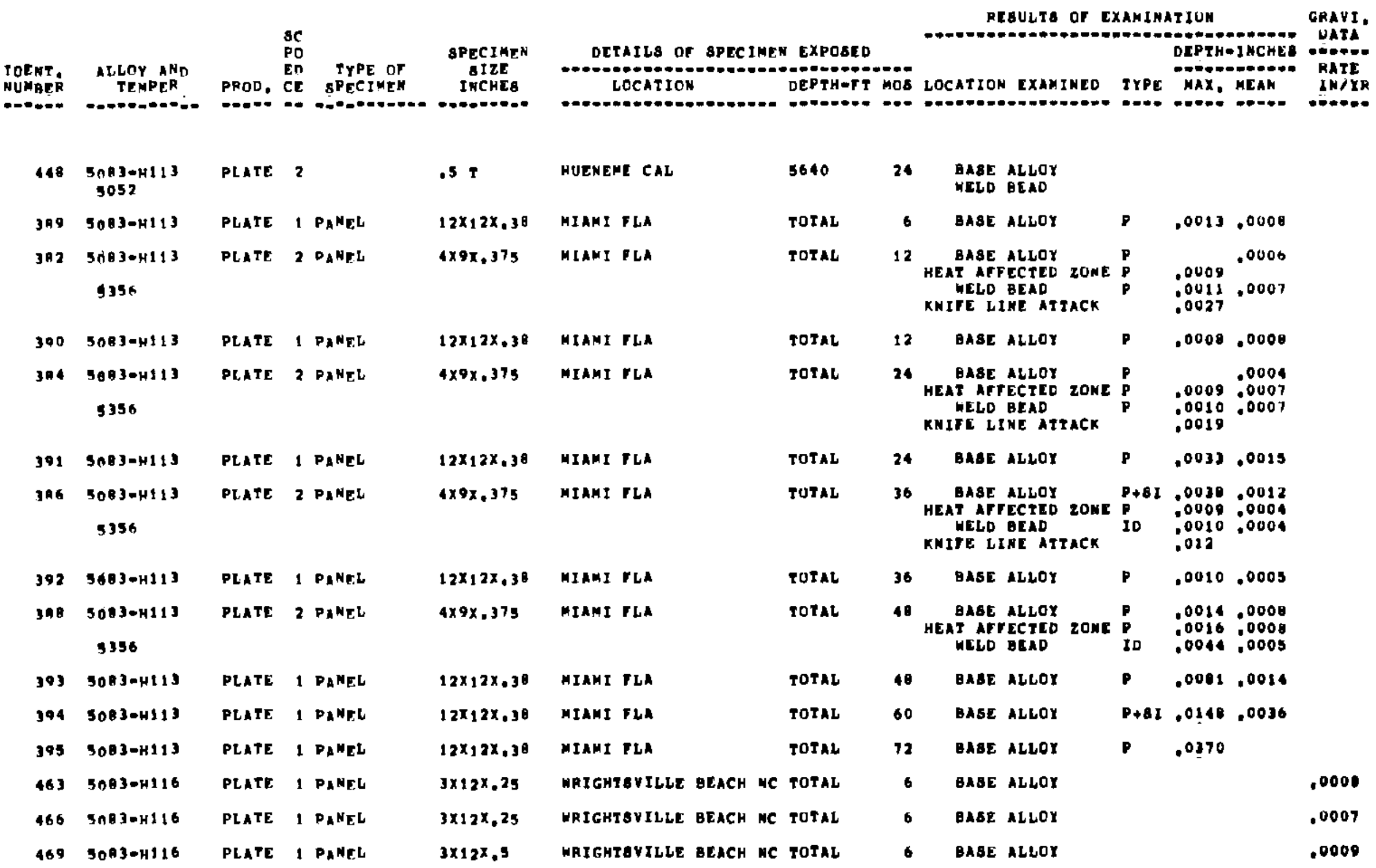


TABLE I

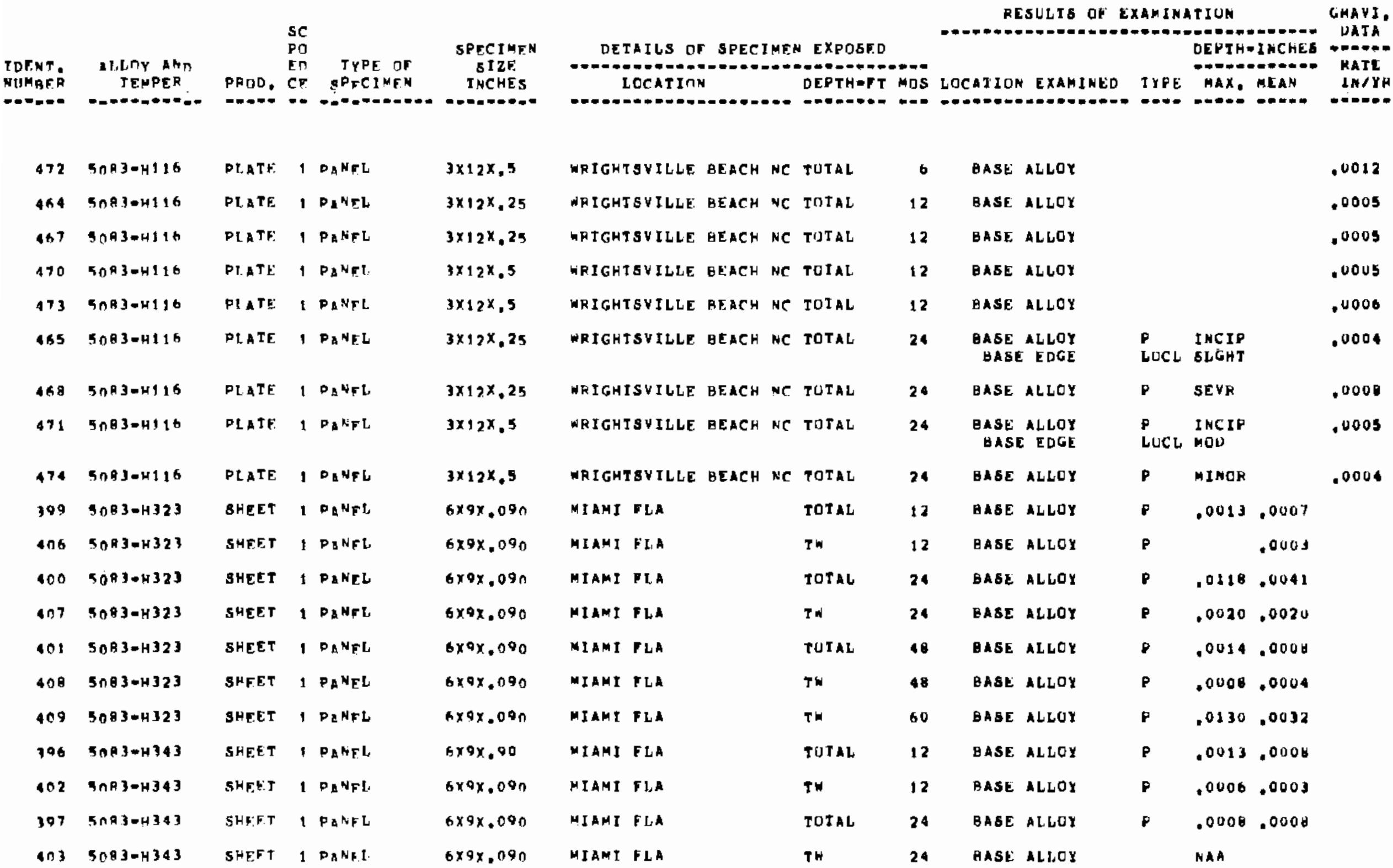


TABLE I

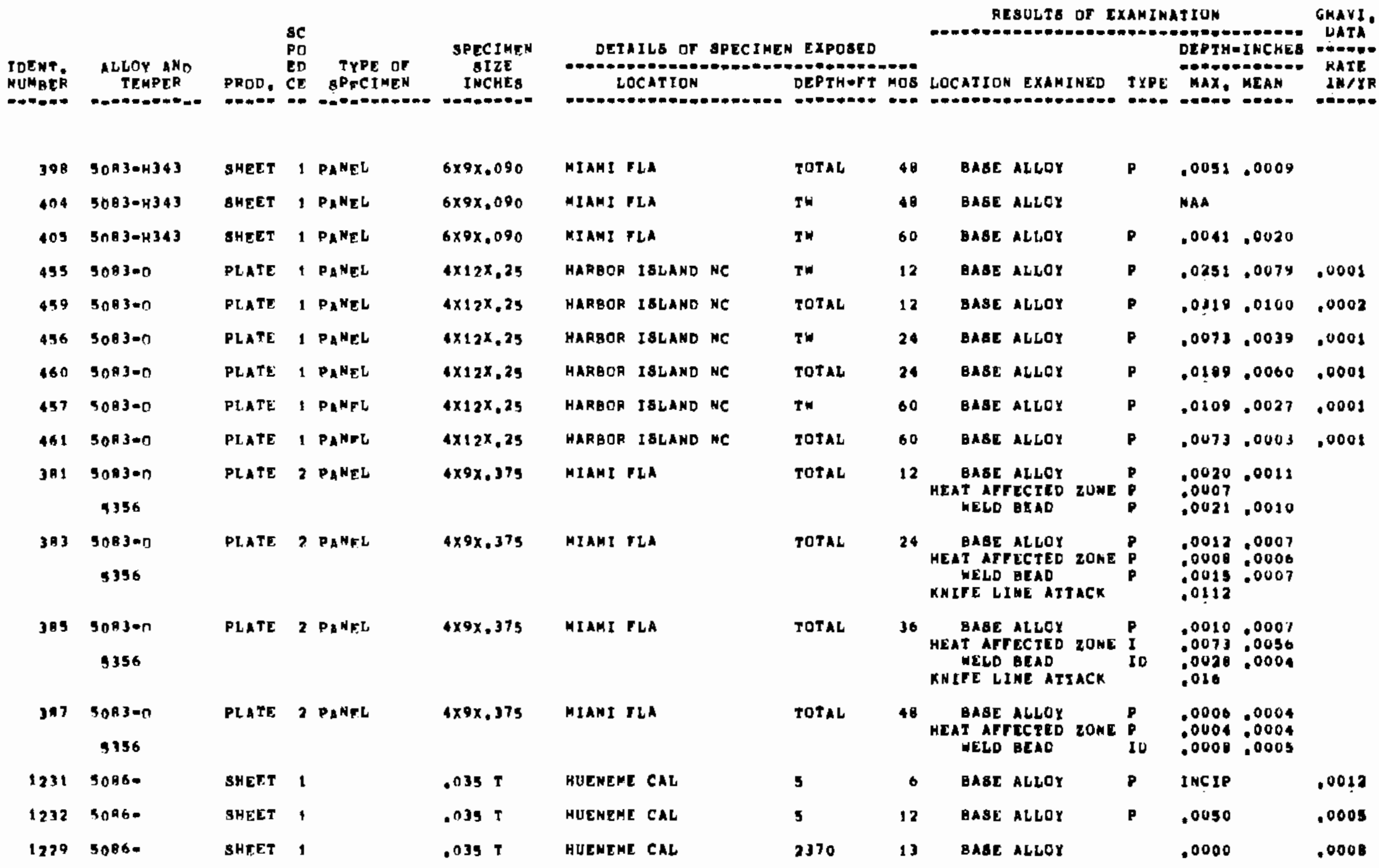


TABLE I

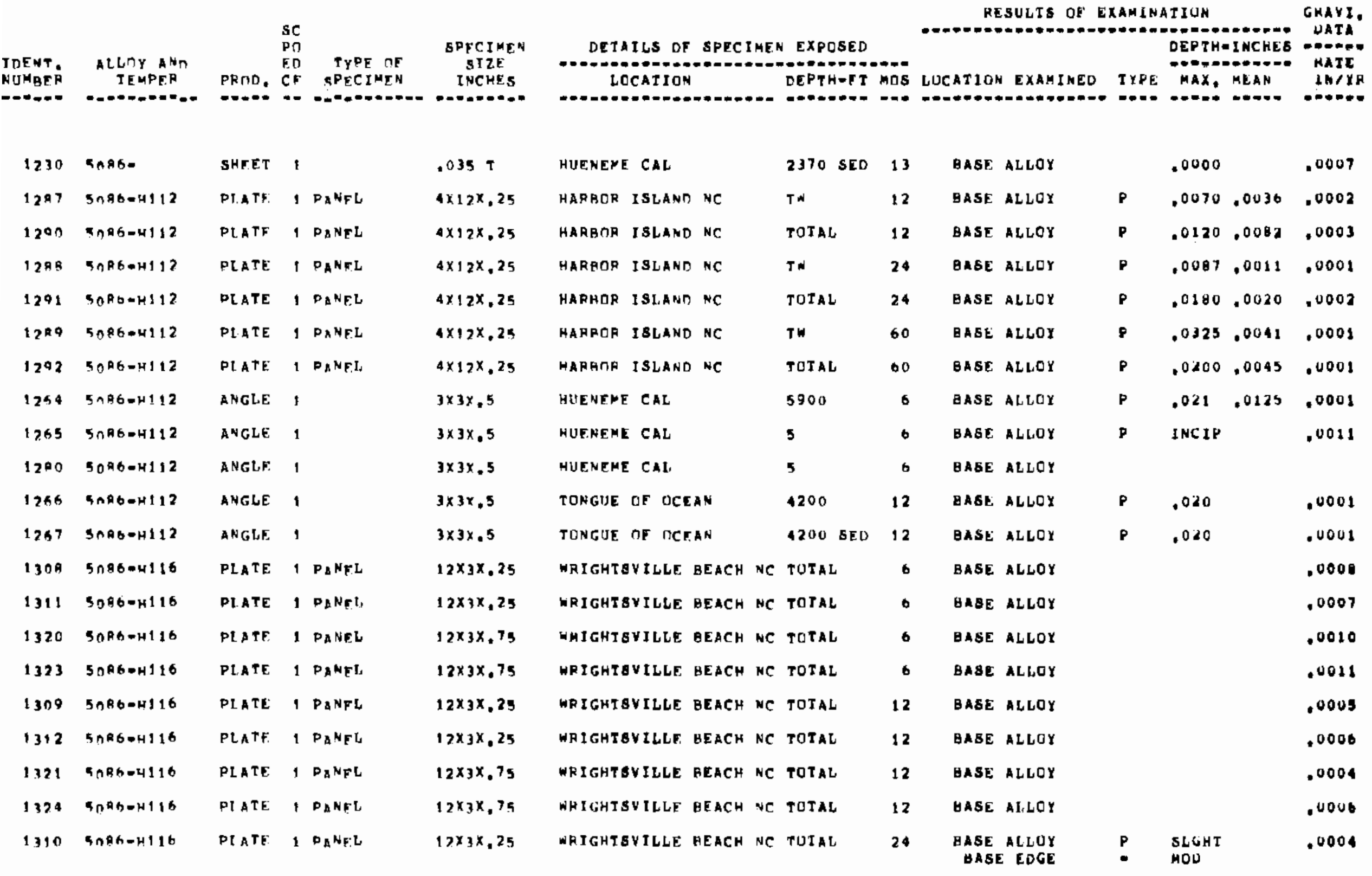


TABLE 1

\begin{tabular}{|c|c|c|c|c|c|c|c|c|c|c|c|c|c|c|}
\hline \multirow{3}{*}{$\begin{array}{l}\text { IDENT, } \\
\text { NUMBR } \\
1313\end{array}$} & \multirow{3}{*}{$\begin{array}{l}\text { ALLDY AND } \\
\text { TEMPER } \\
5096=H 116\end{array}$} & \multirow{3}{*}{ PLATE } & \multirow{3}{*}{$\begin{array}{l}\text { SC } \\
\text { PO } \\
\text { ED } \\
C E \\
-- \\
\\
\\
I F\end{array}$} & \multirow{3}{*}{$\begin{array}{l}\text { TYPE OF } \\
\text { PPRCIMEN } \\
\text { PANFL }\end{array}$} & \multirow{3}{*}{\begin{tabular}{c} 
SPECIMEN \\
AIZE \\
INCHES \\
\hdashline$\ldots=0$ \\
$12 \times 3 \times, 25$
\end{tabular}} & \multirow{3}{*}{$\begin{array}{l}\text { DETAILS OF } \\
\text { WRIGHTSVILLE }\end{array}$} & \multirow{3}{*}{ BEACH NC } & \multirow{3}{*}{ 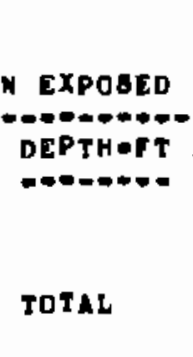 } & \multirow{3}{*}{$\begin{array}{l}-\infty 08 \\
24\end{array}$} & \multirow{2}{*}{$\begin{array}{l}\text { RESULTS OF } \\
\text { ATION EXAMINED } \\
\end{array}$} & \multirow{3}{*}{$\begin{array}{l}\text { TYPE } \\
\text { UNI }\end{array}$} & \multirow{2}{*}{\multicolumn{2}{|c|}{ 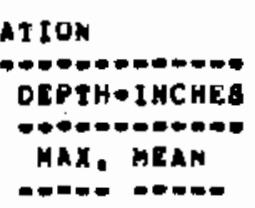 }} & \multirow{2}{*}{ 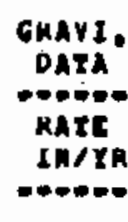 } \\
\hline & & & & & & & & & & & & & & \\
\hline & & & & & & & & & & $\begin{array}{l}\text { BASE ALLOX } \\
\text { BASE EDGE }\end{array}$ & & SLGHT & & .0004 \\
\hline 1322 & $5086-\mathrm{H} 116$ & PLATE & 11 & PANEL & $12 \times 3 \times .75$ & WRIGHTSVILLE & BEACH NC & TOTAL & 24 & $\begin{array}{l}\text { BABE MLLOY } \\
\text { BABE EDGE }\end{array}$ & $\begin{array}{l}\text { UMI } \\
\text { LOCL }\end{array}$ & BLGHT & & .0003 \\
\hline 1325 & $5086=H 116$ & PLATE & 11 & PANEL & $12 \times 3 \times, 75$ & WRIGHTSVILLE & BEACH NC & TOTAL & 24 & $\begin{array}{l}\text { BASE ALLOY } \\
\text { BABE EOGE }\end{array}$ & UNI & MOU & & .0004 \\
\hline 1314 & $5086=H^{117}$ & PLATE & 1 & PaHEL & $8 \times 3 \times, 25$ & WRIGHTSVILLE & BEACH NC & TOTAL & 6 & BASE ALLOY & & & & .0000 \\
\hline 1317 & 50 O००H117 & PLATE & 1 & PANEL & $8 \times 3 \times .25$ & WRIGHTAVILLE & BEACH NC & TOTAL & 0 & BASE ALLOY & & & & .0000 \\
\hline 1315 & $5006-\mathrm{H} 117$ & PLATE. & 1 & PANEL & $9 \times 3 \times .25$ & WRIGHISVILLE & BEACH NC & TOTAL & 12 & BABE ALLOX & & & & .0005 \\
\hline 1340 & $5086-H 117$ & PLATE & I & PaNel & $8 \times 3 \times, 25$ & WFIGHTAVILLE & BEACH NC & TOTAL & 12 & BASE ALLOY & & & & .0005 \\
\hline 1316 & 50 SG $=\mathrm{H} 117$ & PLATE & 1 & PANEL & $8 \times 3 \times, 25$ & WRIGHTSVILLE & BEACH NC & TOTAL & 24 & $\begin{array}{l}\text { BABE ALLOY } \\
\text { BABE EDGE }\end{array}$ & UN1 & NAA & & .0004 \\
\hline 1319 & $5086=4117$ & PIATE & 11 & PaNEL & $8 \times 3 \times .25$ & WRIGHTAVILLE & BEACH HC & TOTAL & 24 & $\begin{array}{l}\text { BASE ALLOY } \\
\text { BASE EDGE }\end{array}$ & $u_{n+}$ & NAA & & .0004 \\
\hline 1,25 & $\begin{array}{l}5086-118 \\
5356\end{array}$ & BHEET & 2 & PANEL & $3 \times 12 \times, 2$ & SKAGEAAK & & TOTAL & 54 & $\begin{array}{l}\text { BABE ALLQY } \\
\text { WELD BEAO }\end{array}$ & & .0000 & & \\
\hline 1233 & $3086-\mathrm{H} 32$ & SHEET & $i$ & & $.063 \mathrm{~T}$ & HUENEME CAL & & 5900 & 6 & GASE ALLOX & $p$ & .033 & .0192 & .0001 \\
\hline 1234 & $30860 \mathrm{H32}$ & SHEET & 1 & & $.065 T$ & HUENEME CAL & & $5900 \mathrm{SED}$ & 6 & BASE ALLOY & $\mathbf{P}$ & .023 & .0169 & .0002 \\
\hline 1237 & $5086-432$ & SHEET & 1 & & $.065 \mathrm{~T}$ & HUENEME CAL & & 2340 & 6 & BABE ALLOY & $\mathbf{p}$ & .0050 & & .0001 \\
\hline 1240 & $3096-N 32$ & SHEET & 1 & & $.065 t$ & HUENEME CAL & & $\mathbf{s}$ & 6 & $\begin{array}{l}\text { GASE ALLOY } \\
\text { BASE LDGE }\end{array}$ & ? & $\begin{array}{l}\text { INCIP } \\
\text { Yro }\end{array}$ & & .0010 \\
\hline 1271 & $5016-N 32$ & SHEET & 1 & & $.065 \mathrm{~T}$ & HUENEME CAL & & 5 & 6 & BABE ALLOY & & & & \\
\hline 1238 & $5086-432$ & EFT & 1 & & $.065 \mathrm{~T}$ & hueneme cal & & 2370 & 13 & BASE ALLOX & $\mathbf{p}$ & INC IP & & .0004 \\
\hline 1239 & $3006-432$ & SHYET & 1 & & $.065 \mathrm{~T}$ & HUENEME CAC & & $2370 \mathrm{st}$ & 13 & BASE ALLOX & $\mathbf{p}$ & INCIP & & .0005 \\
\hline
\end{tabular}


TABLE I

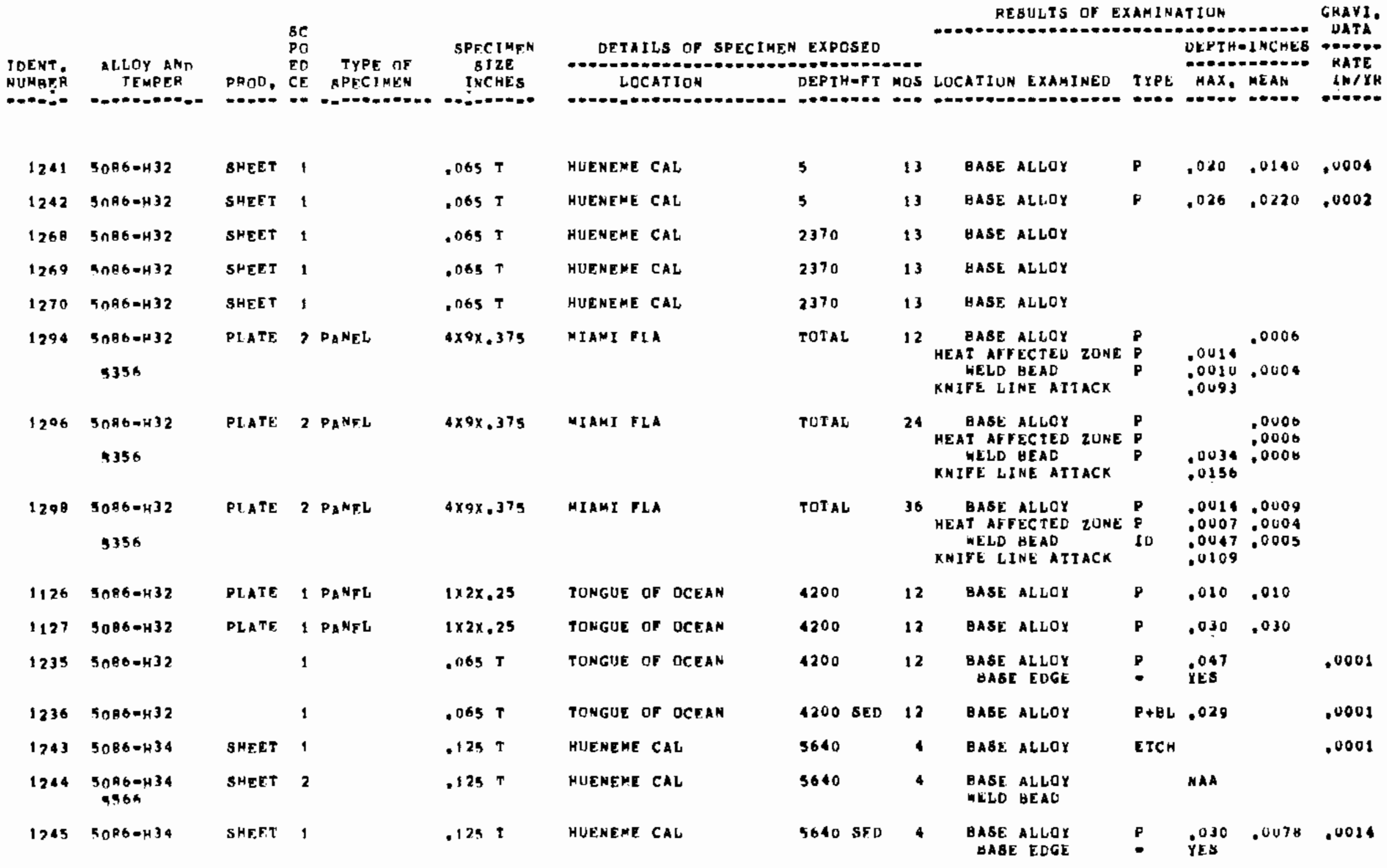


TABLE I

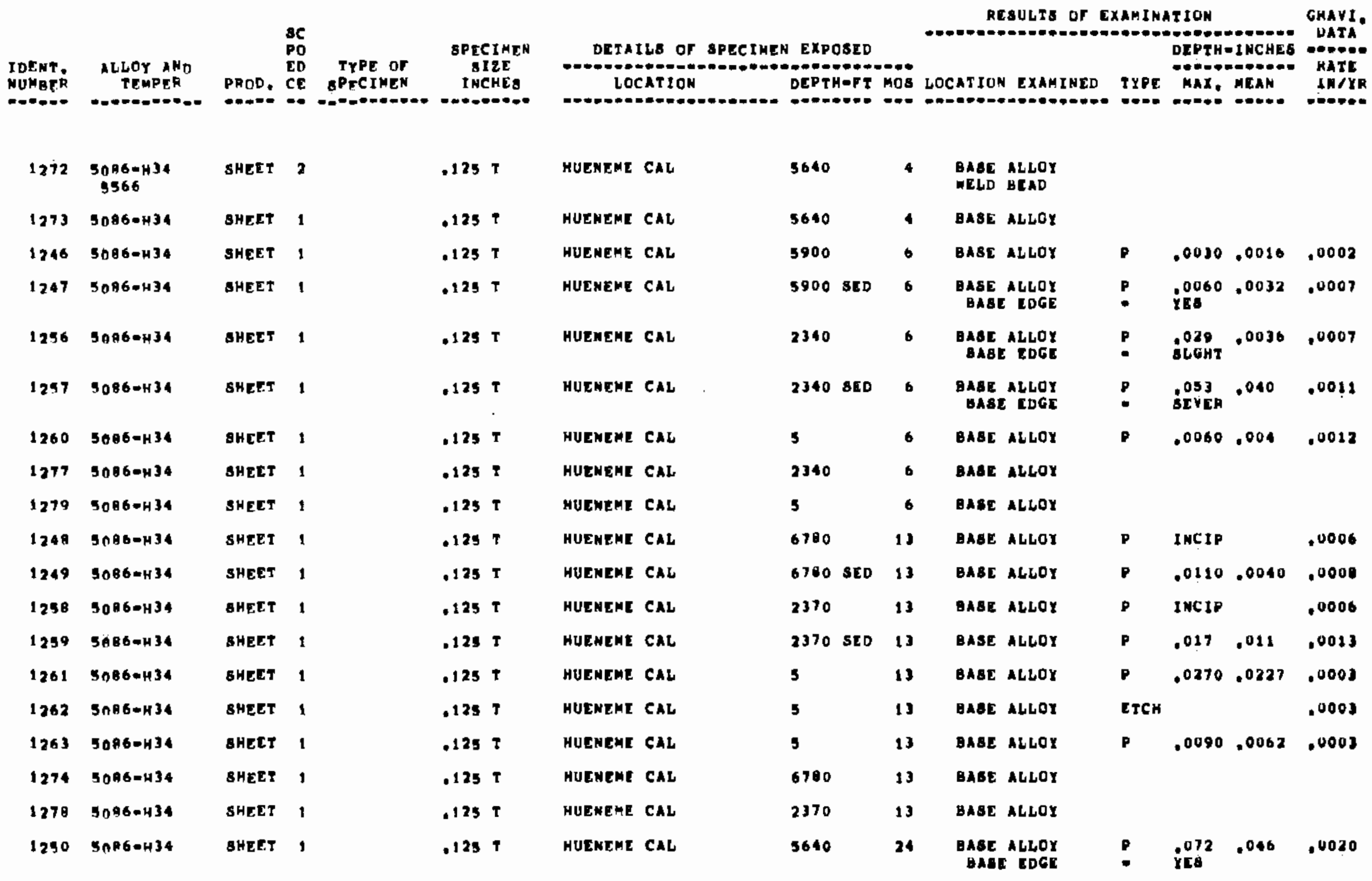


TABLE I

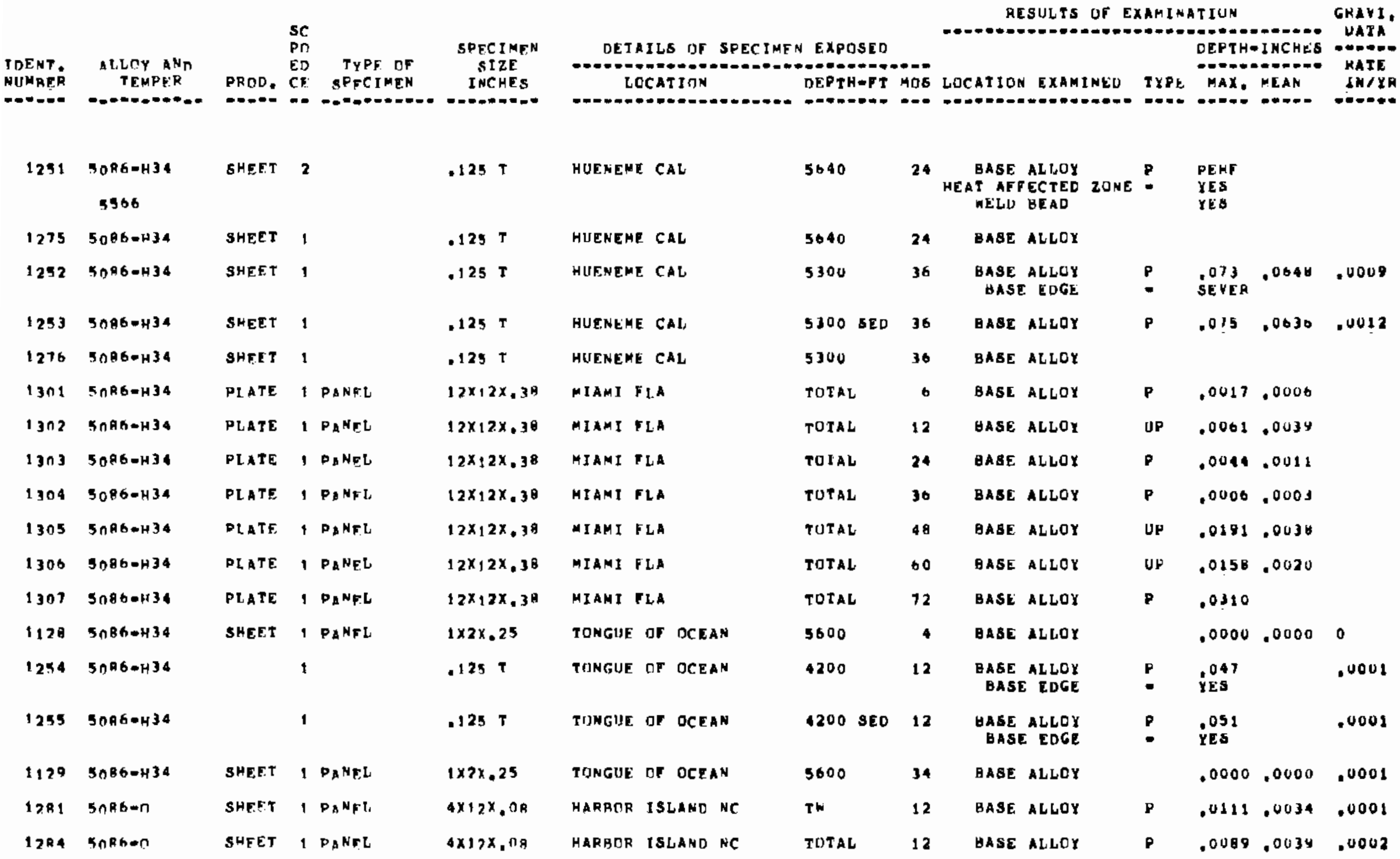


TABLE I

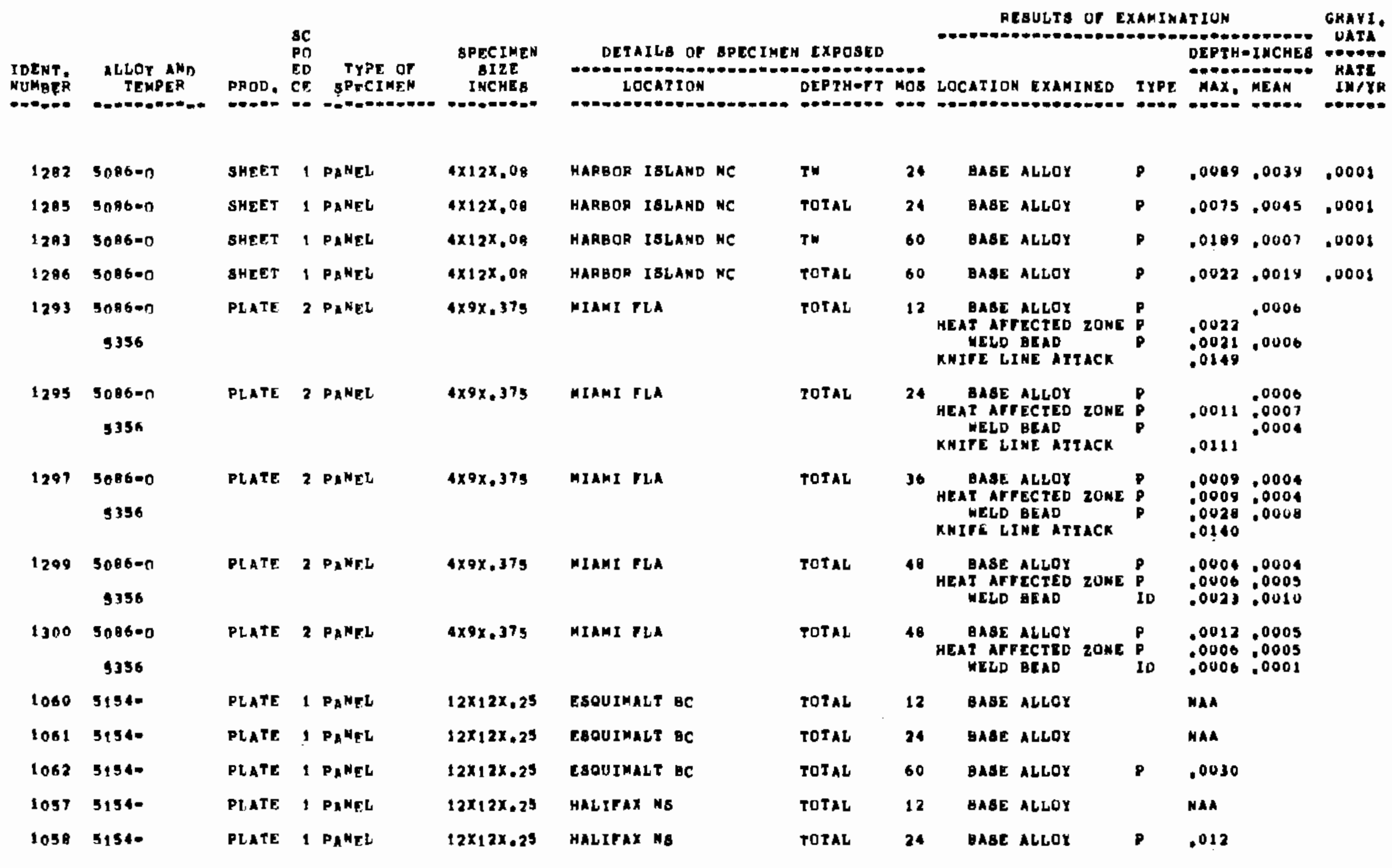


TAELE I

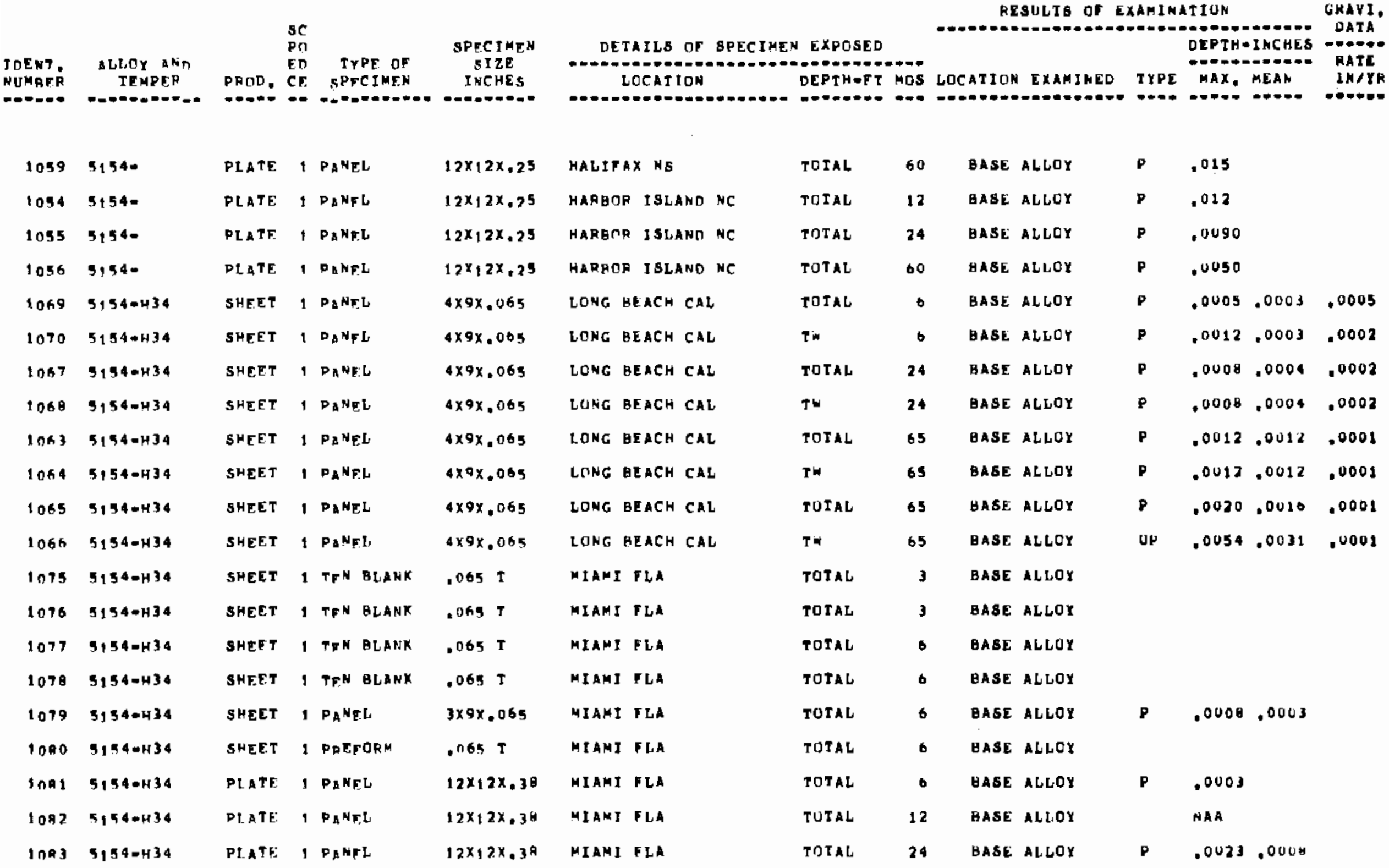


TABLE I

\begin{tabular}{|c|c|c|c|c|c|c|c|c|c|c|c|c|c|c|}
\hline $\begin{array}{l}\text { IDENT: } \\
\text { NUMBER } \\
\text { O- }\end{array}$ & $\begin{array}{c}\text { MLLOY AND } \\
\text { TEMPER } \\
\text { TEME }\end{array}$ & PFOD, & $\begin{array}{l}S C \\
\text { PO } \\
\text { ED } \\
\text { CE } \\
-\infty\end{array}$ & $\begin{array}{c}\text { TYPE OF } \\
\text { SPRCIMEN } \\
\text { PTE }\end{array}$ & $\begin{array}{c}\text { SPECIMEN } \\
\text { SIZE } \\
\text { INCHES } \\
0.0-0.00\end{array}$ & $\begin{array}{l}\text { DETAILS DF BPECIMEN } \\
\text { LCCATION }\end{array}$ & 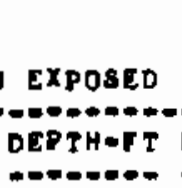 & MOS & LOCATION & EXAMINED & $\begin{array}{l}\text { XAMINA } \\
\text { TYPE } \\
\text { TXOEO }\end{array}$ & $\begin{array}{l}\text { TION } \\
\text { DEPSH } \\
\text { MAX, } \\
=0-0\end{array}$ & $\begin{array}{l}\text { INCHES } \\
\text { MEAN } \\
-E-\infty\end{array}$ & 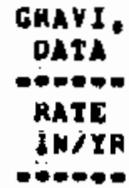 \\
\hline 1084 & $5654-H 34$ & PLATE & 1 & PANEL & $12 \times 12 \times, 38$ & MIAMI FL & TOTAL & 36 & BASE & ALLOY & $\mathbf{p}$ & .0005 & .0002 & \\
\hline 1005 & $5154 * \mathrm{H}_{34}$ & PLate & 1 & PANEL & $12 \times 12 \times, 30$ & MIAMI FLA & Forat & 48 & BABE & ALLOY & $\mathbf{p}$ & .0000 & .0003 & \\
\hline 1096 & $5154=434$ & PIATE & 1 & Pa NeL & $12 \times 12 \times, 38$ & MIAMI FLA & TOTAL & 60 & BASE & ALLOY & $U P+E$ & .0045 & $.002 \mathrm{~b}$ & \\
\hline 1087 & $5154-H_{34}$ & PLATR & 1 & PANEL & $12 \times 12 \times, 38$ & NIAMI ILA & TOTAL & 72 & BAGE & ALLOY & $8 \mathrm{~L} 18$ & .0090 & & \\
\hline $108 \theta$ & $5154 \cdot H 38$ & SHEET & 1 & PRERL & $4 \times 12 \times .05$ & HARBOR ISLAND NC & $\mathbf{T}=$ & 12 & BA8E & ALLOY & $\mathbf{P}$ & .0036 & .0015 & .0001 \\
\hline 1091 & $5154-H 30$ & SHEET & 1 & PANEL & $4 \times 12 \times, 05$ & HAREOP ISLAND NC & TOTAL & 12 & BASE & ALLOY & $\mathbf{P}$ & .0152 & .0035 & .0002 \\
\hline 1089 & $5154-N 38$ & SHECT & 1 & PANEL & $4 \times 12 \times, 05$ & HAREOR ISLAND HC & TH & 24 & BASE & ALLOX & $\mathbf{P}$ & .0051 & .0025 & .0001 \\
\hline 1092 & $5154-H 3 B$ & SHEET & 1 & PA NEL & $4 \times 12 \times, 05$ & HAREOR ISLAND WC & TOTAL & 24 & BASE & ALLOX & $\mathbf{P}$ & .0020 & .0031 & .0002 \\
\hline 1090 & $5154-H 38$ & SHECT & 1 & PANEL & $4 \times 12 \times, 05$ & HARBOR ISLAND HC & $T w$ & 60 & AASE & ALLOX & $\mathbf{P}$ & .0289 & .0010 & .0001 \\
\hline 1093 & $5154-H 38$ & SHEET & 1 & PANFL & $4 \times 12 \times, 05$ & HARBOP ISLAND NC & TOTAL & 60 & BABL & ALLOY & $\mathbf{P}$ & .0102 & .0003 & .0001 \\
\hline 1071 & $5154-0$ & SHEET & 1 & TFN BLANK & $.065 \mathrm{~T}$ & MIAMI FLA & TOTAL & 3 & BASE & ALLOX & & & & \\
\hline 1072 & $5154-0$ & SHEET & 1 & TFH BLANK & $.065 \mathrm{~T}$ & MIAMI FLA & TOTAL & 6 & BASE & ALLOY & & & & \\
\hline 1073 & $5 \pm 54=0$ & SHEET & 1 & Pa AEL & $3 \times 9 \times .065$ & MIAMI FLA & TOTAL & 6 & BABE & ALLOY & $\mathbf{p}$ & .0020 & .0003 & \\
\hline 1074 & $5154=0$ & SHEET & 1 & PAEFORM & $.065 \mathrm{~T}$ & MIAMI FLA & TOTAL & 6 & BASE & ALLOY & & & & \\
\hline 1052 & $5254-H 34$ & SHEET & 1 & Panel & $3 \times 9 \times, 065$ & HUENEWE CAL & 5100 & 35 & BABE & ALLOY & $\mathbf{P}$ & PEHT & & .0001 \\
\hline 1053 & $5954-H^{34}$ & PLATE & 1 & $C \oplus$ ING & .75 DIA & HUENTPE CAL & 5100 & 35 & SABE & ALLOY & & & & \\
\hline 1046 & $5854-H^{34}$ & SHEET & $t$ & PRNEL & $3 \times 9 \times .065$ & MIAMI FLA & $T W$ & 6 & 8ASE & ALLOY & $\mathbf{P}$ & .0004 & .0004 & \\
\hline 1047 & $5,54-\mathrm{H}^{34}$ & SHEET & 1 & PANEL & $3 \times 9 \times .065$ & MIANI TLA & TOTAL & 6 & GABC & ALLOY & & waA & & \\
\hline 1049 & $5254-H 34$ & PLATE & 1 & e-RING & .75 DIA & MIAMI TLA & $7 H$ & 6 & BASE & ALLOY & & & & \\
\hline 1050 & $5254-H_{34}$ & PLATE & 1 & $C \cdot R I N G$ & .75 DIA & MIAMI FLA & TOTAL & 6 & BASE & ALLOX & & & & \\
\hline 1048 & $5,54-H^{34}$ & SHEET & 1 & PANEL & $3 \times 9 \times .065$ & TONGUE OF OCEAN & 5100 & 4 & BASE & ALLOY & & MAA & & \\
\hline
\end{tabular}


TABLE 1

IDENT. TLIOY AND

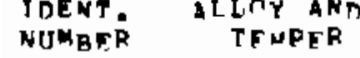
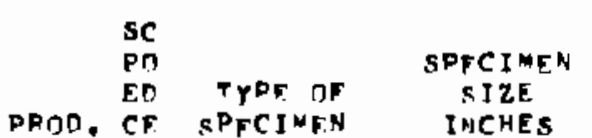

PRDO. CP SPFCINFN

INCHES

\begin{tabular}{|c|c|c|c|c|}
\hline 1051 & $5254=134$ & PLATE & 1 & CARINA \\
\hline $10 \mathrm{A4}$ & $5356=$ & SHEET & 1 & PANFL \\
\hline 1045 & $5356=$ & SHEET & 1 & PANFL \\
\hline 1343 & 54540 & SHEET & 1 & \\
\hline 1344 & $2454=$ & SHEET & 1 & \\
\hline 1341 & 94540 & SHEET & 1 & \\
\hline 1342 & 94240 & SHEET & 1 & \\
\hline 1333 & $5454-1432$ & PI ATE & 1 & PANEL \\
\hline 1337 & $5454-132$ & PLATE & 1 & PANEL \\
\hline 1334 & $3454-432$ & PLATE & 1 & PANEL \\
\hline 1319 & $5,54-432$ & PL ATE & 1 & $P_{A} N_{E L}$ \\
\hline 1335 & $5454-432$ & PLATE & 1 & PANET \\
\hline 1336 & $\begin{array}{c}5454=1432 \\
\$ 554\end{array}$ & PIATE & 2 & PaNFL \\
\hline 1339 & $5454-432$ & PI ATE & 1 & PaNEL \\
\hline 1340 & $5454-H 32$ & PI ATE. & $?$ & PANFL \\
\hline
\end{tabular}

.75 DIA TONGIJE OF OCEAN

$4 \times 9 \times, 065$

MIAMI FLA

$4 \times 9 \times .065$

NIAMI FLA

- 0 ค $T$

HUENEHE CAL

- 0 B $T$

. 0 ค $\mathrm{T}$

. OA $\mathrm{T}$

HUENEME CAL

HLFNEME CAL

HIENEME CAL

$4 \times 12 \times .25$

MARBDP ISLAND NC

$4 \times 12 \times .25$

HARBOA ISLAND NC

$4 \times 12 \times .25$

HARBOF ISLAND NC

$4 \times 12 \times, 25$

HARRDP ISLANA NC

$4 \times 12 \times, 25$

HARROR IBLAND NC

$4 \times 12 \times, 25$

HARROH ISLAND HC

$4 \times 12 \times, 25$

HARBOR ISLAND HC

$4 \times 12 \times .25$

HAPBDF ISLAND NC

3554 
TABLE I

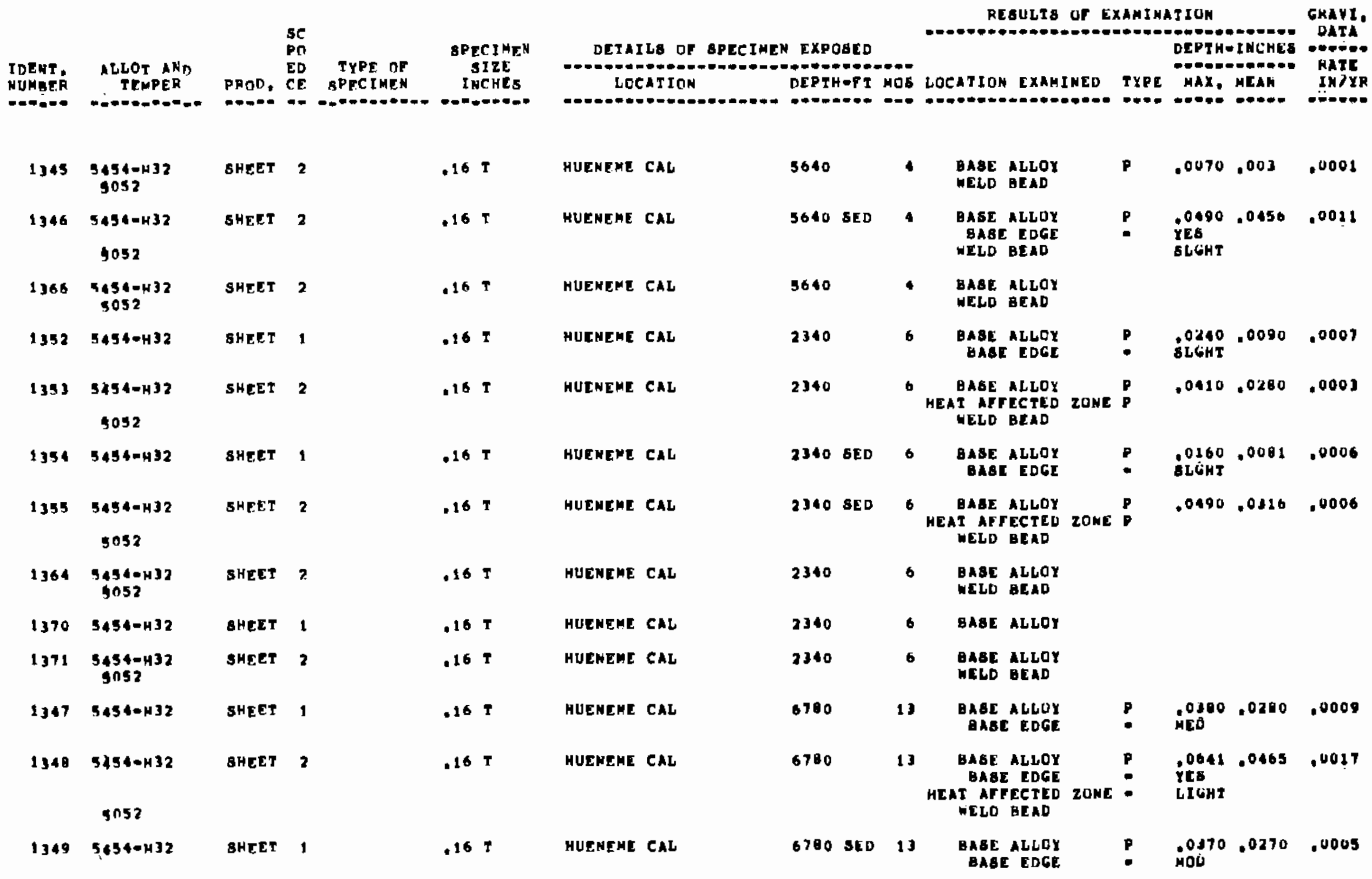


TABLE. I

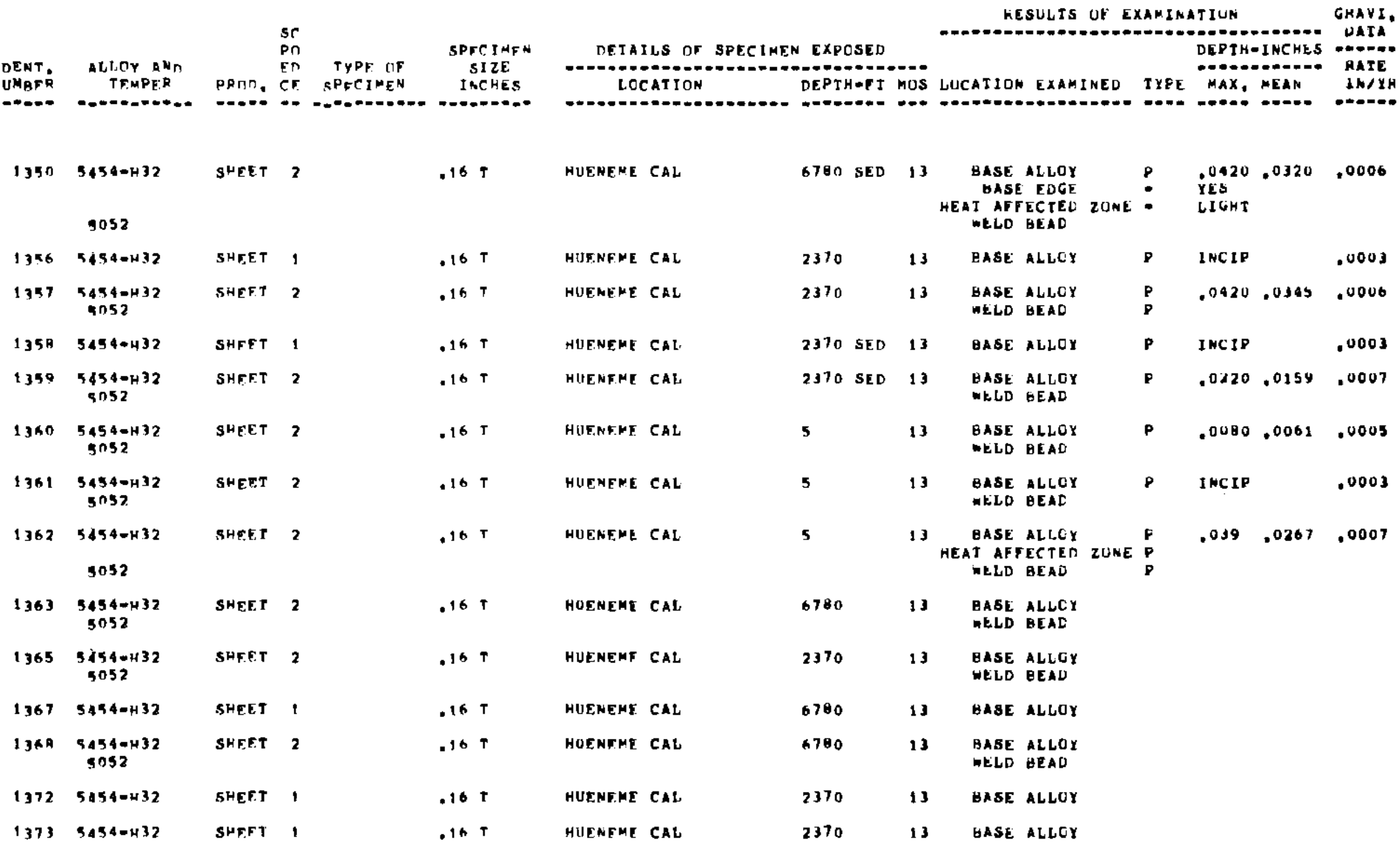


TARLE I

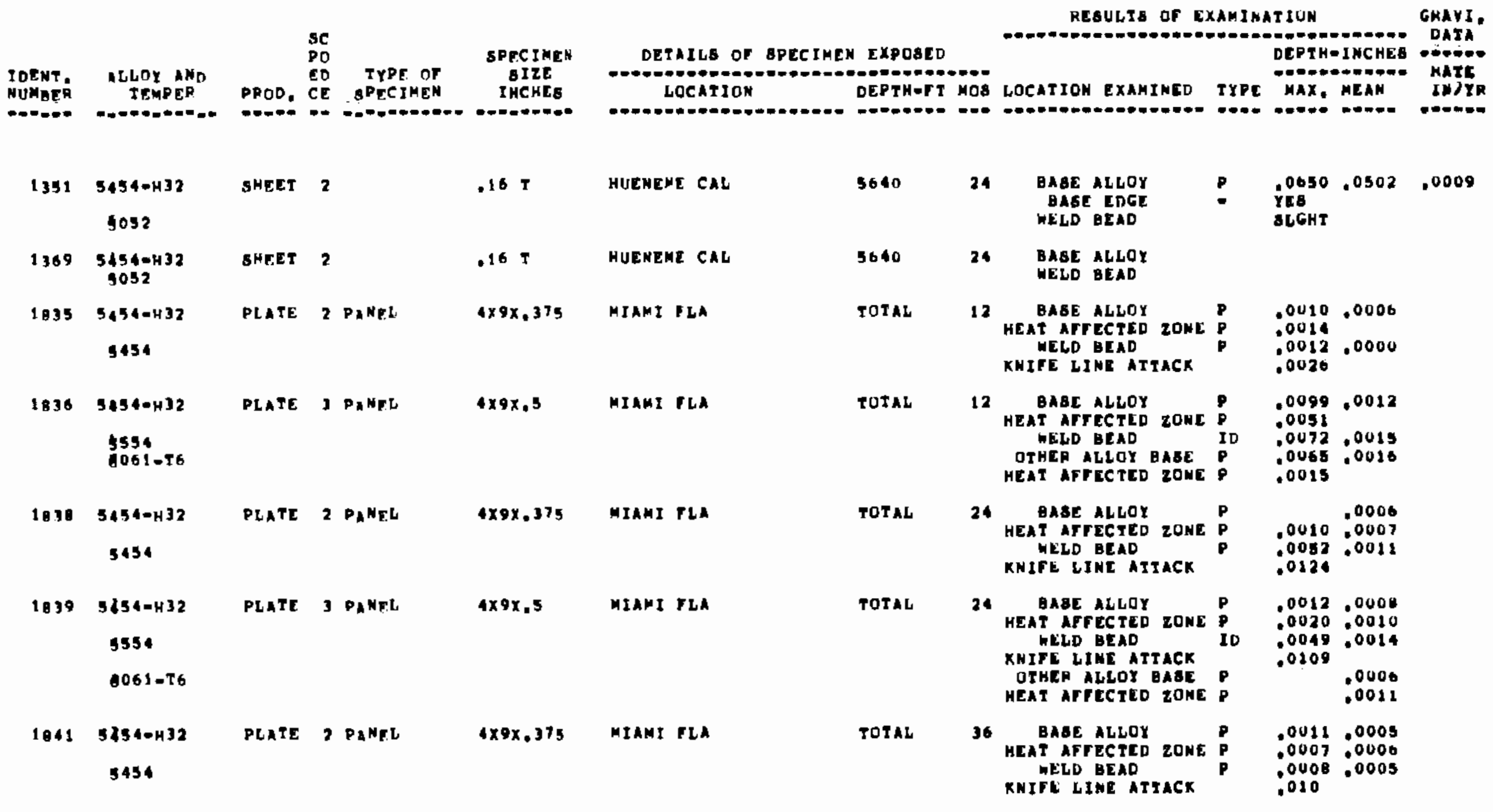


TABLE I

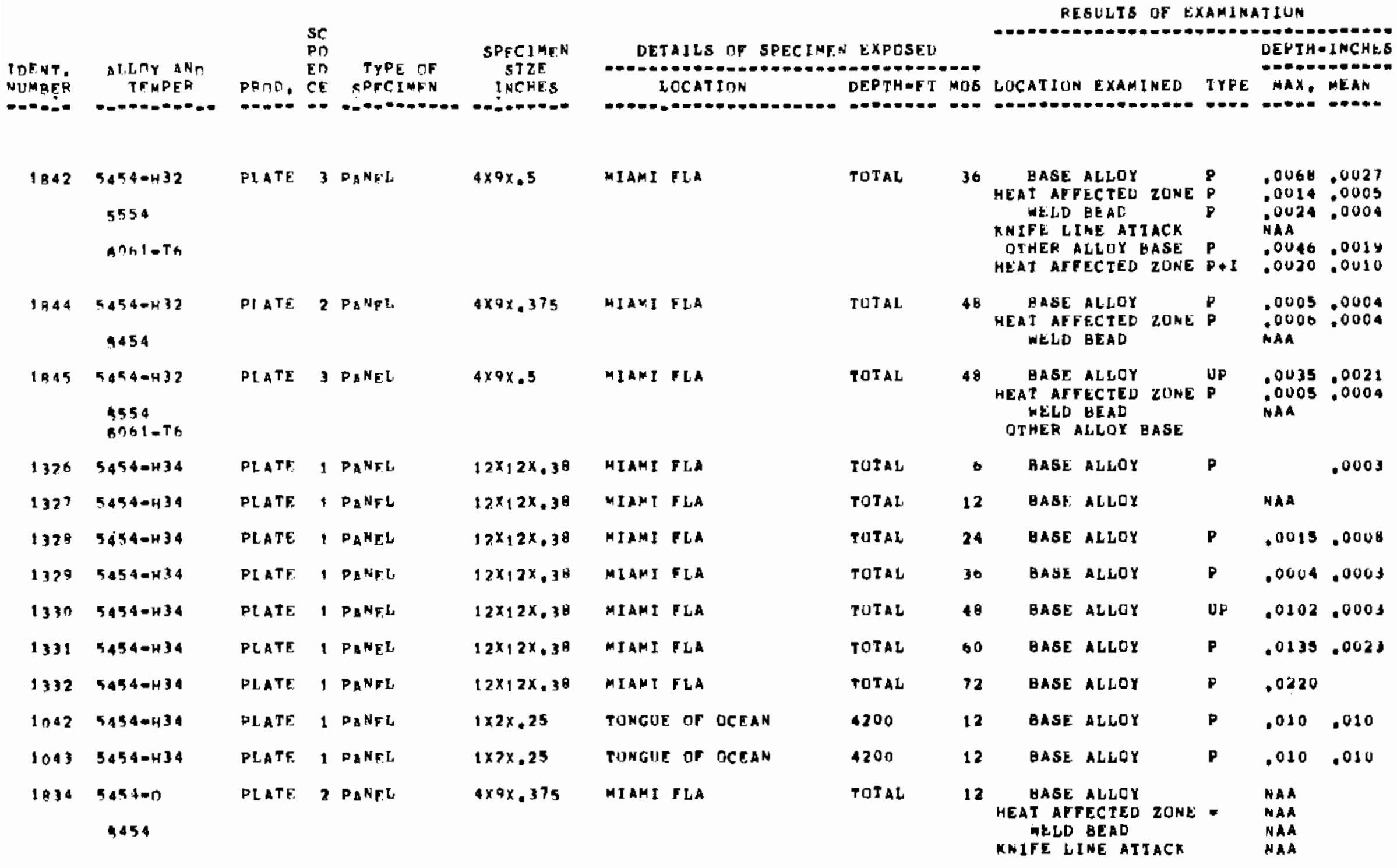


TABLE I

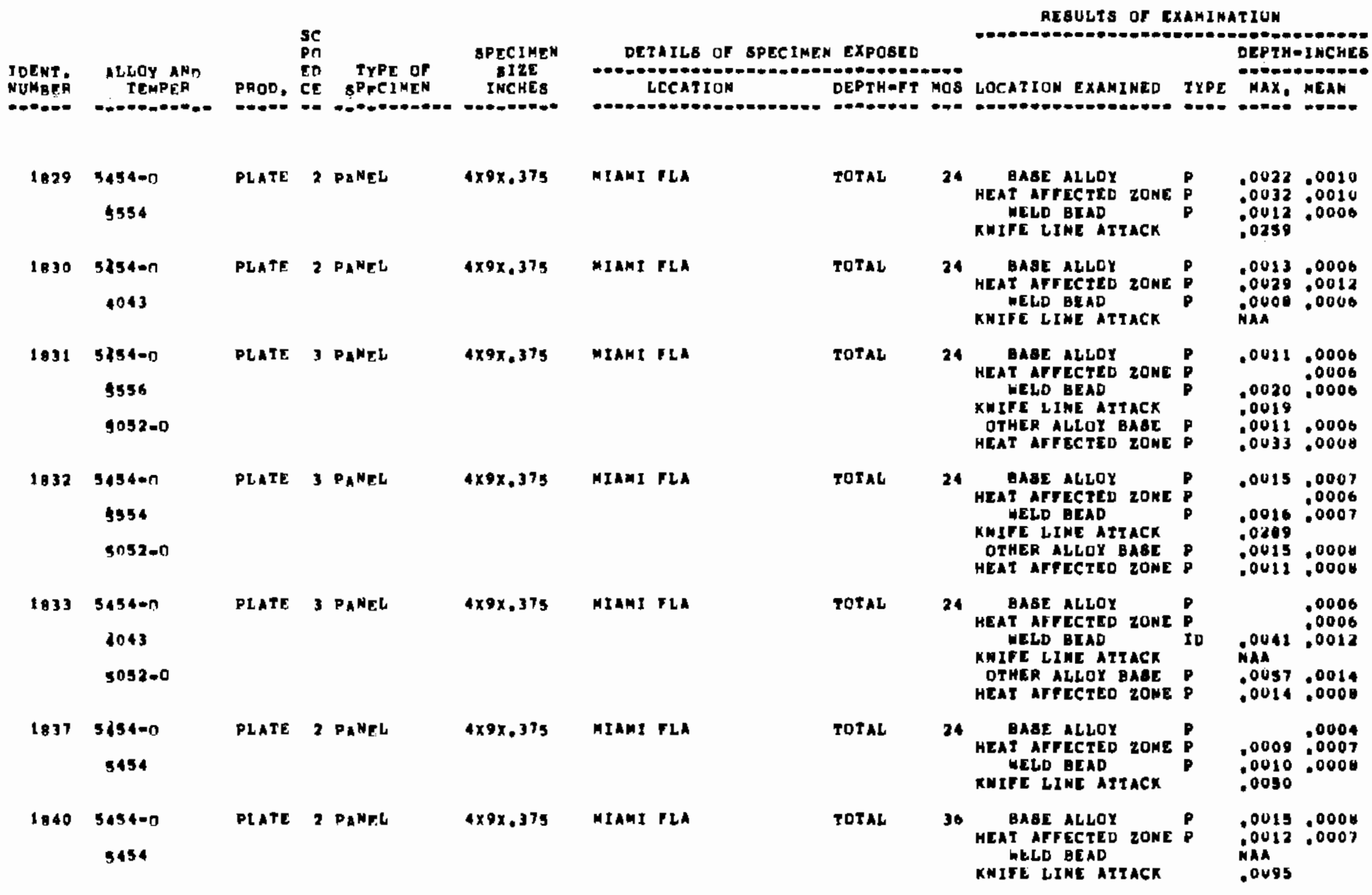


TABLE I

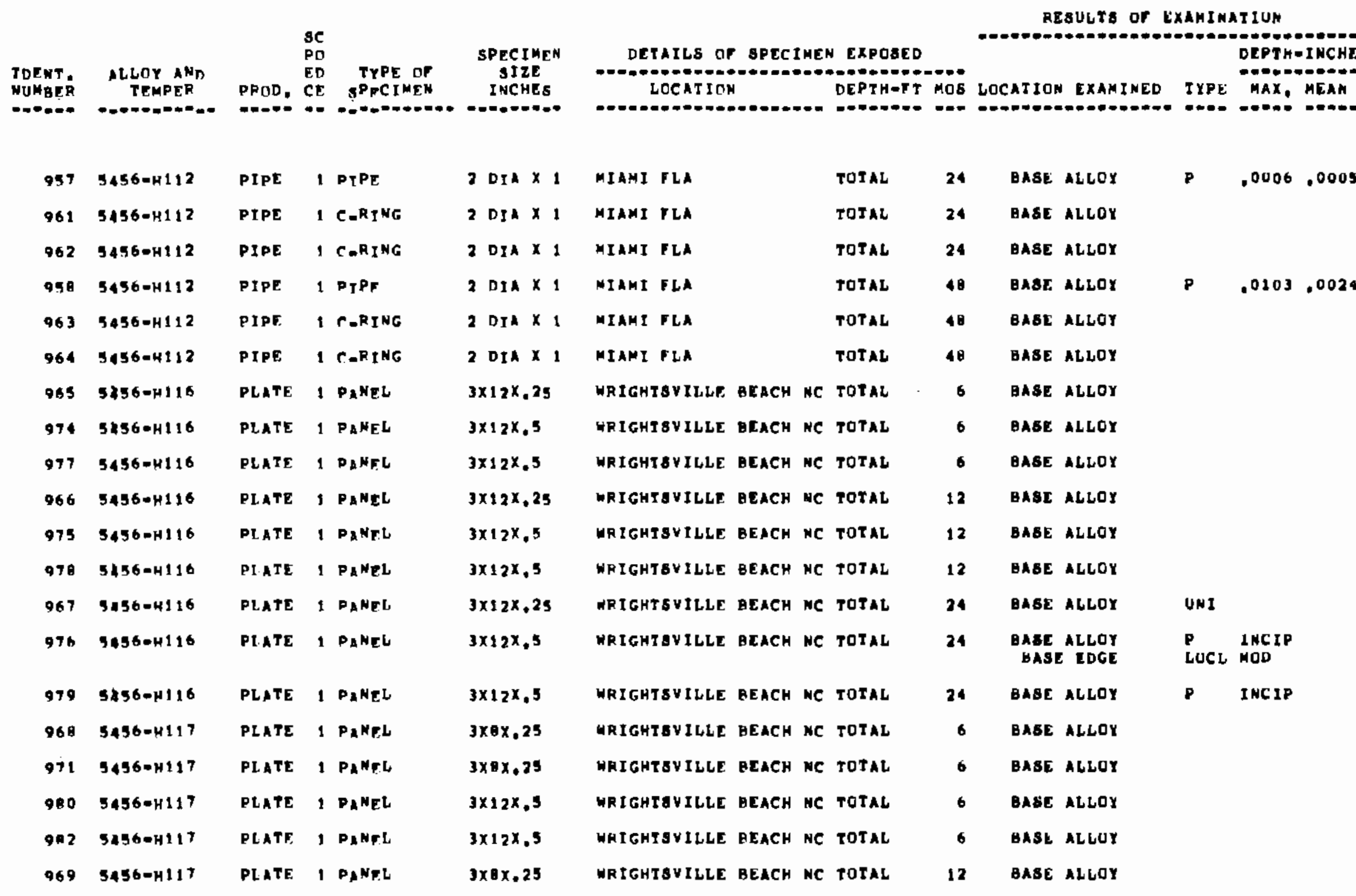

.0008
.0007
.0019
.0005
.0005
.0080
.0004
.0005
.0012
.0007
.0007
.0006
.0000
.0005

.0008

.0007

.0019

.0005

.0005

0030

000

0005

0012

.0007

000

.0005 
TAELF. 1

TAFNT. ALLOY ANT NITMER TEMPEF

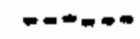

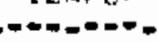

\begin{tabular}{|c|c|c|c|c|}
\hline 072 & $5456-H 117$ & PIATE. & 1 & PANFL \\
\hline QR 1 & $545 b=41 t^{7}$ & PI ATE & 1 & PANFL \\
\hline 993 & $3456=H 117$ & PIATE. & 1 & $P_{\triangle} N F L$ \\
\hline 970 & $5456-\mathrm{H} 117$ & PLATE & 1 & $P_{A} N_{F} t_{1}$ \\
\hline 973 & 545604117 & PL ATF: & 1 & $D_{A} N F L_{J}$ \\
\hline 1421 & $5456-H 14$ & SHEET & 1 & PANFL \\
\hline 1423 & $5456-114$ & SHEET & 1 & $P_{A} N_{F}$, \\
\hline 930 & $5450-424$ & SHEET & 1 & $D_{A} N F I_{2}$ \\
\hline 933 & $5456-H 24$ & SHEET & 1 & $P A^{N} F L$ \\
\hline 934 & $5456-424$ & SHEET & 1 & $D_{A} N_{F} L$ \\
\hline 931 & $5456 \cdot H 24$ & SHEET & 1 & $D_{A} N_{F} L$ \\
\hline 935 & $5456=474$ & SHEET & 1 & $D A M F L$ \\
\hline 932 & $5456-124$ & SHEET & 1 & $P_{A} N_{E L}$ \\
\hline 936 & $5456-H 24$ & SHEET & 1 & $D_{A} N_{T} L$ \\
\hline 097 & $5456-1424$ & SHEET & 1 & PANFL \\
\hline 944 & $5456=H^{3} 11$ & ANGLE & 1 & $\Delta N G_{I}, F$ \\
\hline 035 & $5456-\mu 311$ & ANGLE. & 1 & ANGI,E \\
\hline 1009 & $5456-\mathrm{H} 32$ & & 1 & \\
\hline 1010 & $5456-H 32$ & & 1 & \\
\hline 1040 & $5456-H 32$ & & 1 & \\
\hline 04 & $5450-432$ & & 3 & \\
\hline
\end{tabular}

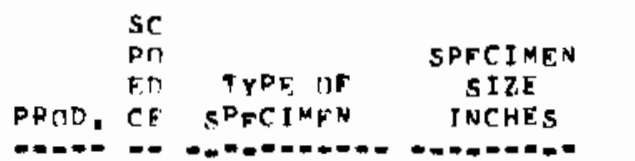

TEIAILS OF SPECIMEN EXPOSED

RESULIS OF EXAMINATIUN GKAYI. - retaivs or SPECIMEN EXPOSED LOCATIDN DEPTHEFT MAS

LUCATION EXAMINEL IYPE MAX.MEAN G IMIYH

\begin{tabular}{|c|c|c|c|c|c|c|c|c|c|c|c|}
\hline $3 \times 8 \times .25$ & WRIGHISVILLE & AEACH & NC & TOTAL & 12 & BASE & ALLOY & & & & .0006 \\
\hline $3 \times 12 \times, 5$ & WFTGHTSVILILE & BEACH & NC & TOTAL & 12 & GASE & ALLOX & UNI & & & .0005 \\
\hline $3 \times 1, \times, 5$ & WRIGHTSVILLE & BEACH & NC: & TOTAL & 12 & AASE & ALLOY & UN 5 & & & .0009 \\
\hline $3 \times 8 \times .25$ & WFIGHISVILLE & DEACH & NC & TOTAL & 24 & BASE: & ALLOY & $U_{N} I$ & & & .0003 \\
\hline $3 \times 9 \times .25$ & AR I GHTSVILLE. & $\mathrm{BEACH}$ & NC & TDIAL & 24 & BASE & ALLOY & UN I & & & .0005 \\
\hline $3 \times 9 \times .065$ & MIAMIFLA & & & TUTAL & 12 & BASE & ALLOY & $\mathbf{P}$ & .0017 & .0000 & \\
\hline $3 \times 9 \times .069$ & $M I A M I F L A$ & & & $T *$ & 12 & BASE & ALLOY & & NAA & & \\
\hline $4 \times 9 \times .064$ & MIAMI FI.A & & & TOTAL & 12 & BASE & ALLOY & $\mathbf{P}$ & .0040 & .0019 & \\
\hline $4 \times 9 \times .064$ & MIAMT FLA & & & TUTAL & 12 & BASE & ALLOY & $\mathbf{p}$ & .0290 & .0038 & \\
\hline $4 \times 9 \times .064$ & MIAMI FLA & & & $\tau *$ & 12 & BASE & ALLOY & $\mathbf{P}$ & .0007 & .0007 & \\
\hline $4 \times 9 \times .064$ & MIAMI FIA & & & TOTAL & 36 & BASE & $\triangle 1 \mathrm{LOY}$ & $\mathbf{P}$ & .0101 & .0010 & \\
\hline $4 \times 9 \times .064$ & MIAHT FLA & & & $T$ in & 36 & BASE & ALLUY & $\mathbf{P}$ & .0010 & .0003 & \\
\hline $4 \times 0 \times .064$ & MIAMI FIA & & & TOTAL & 48 & BASE & ALLOY & $\mathbf{P}$ & .0082 & & \\
\hline $4 \times 9 \times, 064$ & MIAMI FLA & & & Tin & 60 & GASE & ALLOX & $\mathbf{P}$ & .0027 & & \\
\hline $4 \times 9 \times .064$ & MIAHI FIA & & & Th & 72 & HASE & ALLOY & $p$ & .0112 & .0025 & \\
\hline $2 \times 2 \times 12$ & MIAMI FLA & & & $T w$ & 48 & BASE & ALLOY & $I+P$ & .0037 & .0021 & \\
\hline \multirow[t]{5}{*}{$2 \times 2 \times 12$} & MIAMI FL:A & & & TOTAL & 48 & BASE & AILOX & I & .0048 & .0014 & \\
\hline & HUFNEME CAL & & & 2370 & 13 & BASE & ALLOX & & .0000 & & .0006 \\
\hline & HUENEME CAL & & & 2370 SED & 13 & BASE & ALLOY & & .0000 & & .0007 \\
\hline & HUENFME CAL & & & 2370 & 18 & BASE. & ALISOY & & & & \\
\hline & HIIENEME CAI & & & 2370 & 18 & EASE & ALLOY & & & & \\
\hline
\end{tabular}


TABLE I

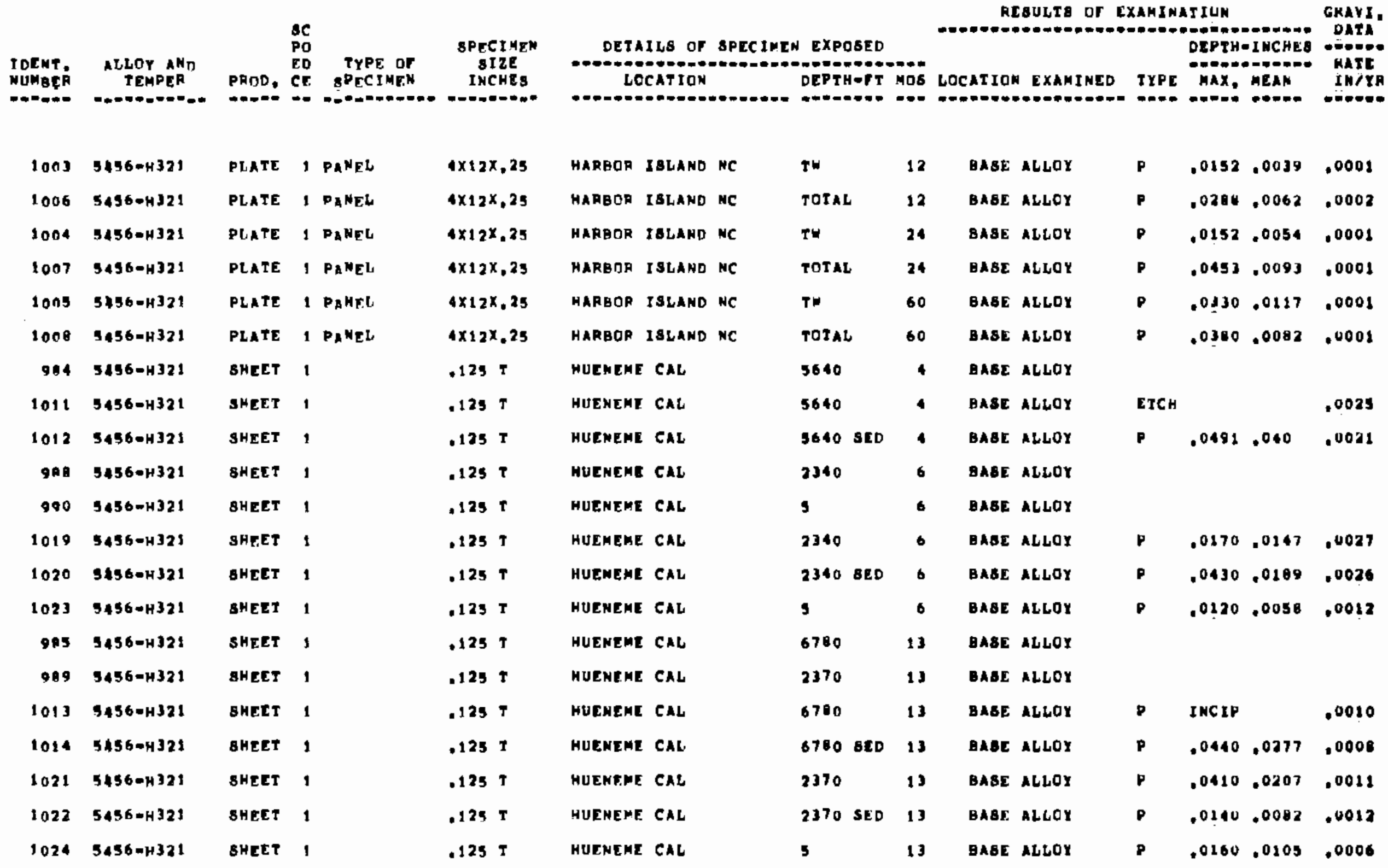


TAALE I

\begin{tabular}{|c|c|c|c|c|c|c|c|c|c|c|c|c|c|c|}
\hline \multirow{2}{*}{$\begin{array}{l}\text { I DENT, } \\
\text { NIJMAFP } \\
\end{array}$} & $\begin{array}{l}\text { TIAY ANn } \\
\text { TTMPER }\end{array}$ & \multicolumn{2}{|c|}{$\begin{array}{r}\text { SC } \\
P ח \\
\text { ED } \\
\text { PROD. CF }\end{array}$} & $\begin{array}{l}\text { TYPF DF } \\
\text { SPFCIMFN }\end{array}$ & $\begin{array}{c}\text { SPFCI AEN } \\
\text { SIZE } \\
\text { INCHES }\end{array}$ & $\begin{array}{l}\text { DETAILS DF SPECIMFN } \\
\text { DESTH }\end{array}$ & \multicolumn{2}{|c|}{ EXPOSED } & LOCATION & ESULTS UF EX & CAMINA & 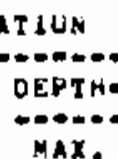 & $\begin{array}{l}\text { IhCHE'S } \\
\text { - MAA }\end{array}$ & $\begin{array}{c}\text { GKAYI, } \\
\text { DATA } \\
\text { MATE } \\
\text { GN/YR }\end{array}$ \\
\hline & $-m--\infty-=-=-\infty$ & $-\infty-\infty$ & $\approx$ & 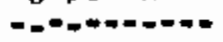 & $--0-0-0-0$ & 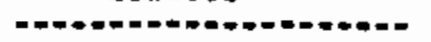 & $-\infty+-\infty-\infty=-$ & $=-$ & ש. & 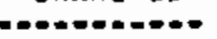 & $\ldots$ & $-\infty+$ & -.... & $-\infty=\infty$ \\
\hline $\ln 29$ & $5456-4321$ & SHEET & 1 & & $.125 T$ & HUENEME CAL & $\mathbf{s}$ & 10 & YASE & ALLOY & $\mathbf{p}$ & .0140 & .0115 & .0008 \\
\hline Oด 6 & $5456-H 321$ & SHEE'T & 1 & & $.125 \mathrm{~T}$ & HUFNEME CAL & 5640 & 24 & GASE & ALLOY & & & & \\
\hline 1015 & $5456-\mathrm{H32}$ & SHEET & 1 & & $.125 \mathrm{~T}$ & HUENEME CAL & 5640 & 24 & BASE & ALGOY & p & .0530 & .0345 & .0020 \\
\hline $\ln 16$ & $9456-1321$ & SHFFT & 1 & & $.125 \mathrm{~T}$ & HUENEME CAL & 3640 SED & 24 & BASE & ALLOY & $\mathbf{P}$ & .0500 & .0313 & .0022 \\
\hline 1419 & $545 b=4321$ & PLATE & 1 & C.. PING $^{-1}$ & $.7501 \mathrm{~A}$ & HUENERE CAL & 5100 & 35 & BASE & ALLOY & & & & \\
\hline 997 & $5856-H 321$ & SHEET & 1 & & $.125 \mathrm{~T}$ & HUENEME CAL & 5300 & 36 & BASE & ALLOY & & & & \\
\hline 1017 & $5456=+321$ & SHEET & 1 & & $.125 t$ & HUENEME CAL & 5300 & 36 & BAEE & ALLOY & $\mathbf{P}$ & .0430 & .0365 & .0013 \\
\hline $1 \cap 18$ & 515 nु- 4321 & SHEF'T & 1 & & $.125 \tau$ & HUENEME CAL & 5300 SED & 36 & BASE & ALLOX & $P$ & .0360 & .0330 & .0015 \\
\hline 923 & $5456-14321$ & DL ATE & 1 & $P_{A} N_{F} I_{1}$ & $12 \times 12 \times 2$ & IIAMI FLA & TLTA A & 6 & BA8E & ALLOY & $\mathbf{P}$ & .0026 & .0010 & \\
\hline 1377 & $5456-1321$ & PLATE & 1 & C.RING & .75014 & MIAMI FLA & $T w$ & 6 & BASE & ALLOY & & & & \\
\hline 1379 & $5456-4321$ & PLATE & 1 & $C D R I N G$ & .75 DIA & MIAMI FLA & TOIAL & 6 & BASE & ALLOY & & & & \\
\hline 1380 & $5656-4371$ & PI ATF & 1 & $D_{4}$ NF.L & $12 \times 12 \times, 39$ & MIAMI FLA & TOTAL & 6 & BASE & ALLOY & $\mathbf{P}$ & .0049 & .0010 & \\
\hline $13 R 7$ & $5456-4321$ & PLATE & 1 & DaNFL & $12 \times 12 \times, 5$ & MIAMI FLA & TOTAL & 6 & BASE & ALLOY & $\mathbf{P}$ & .0167 & .0007 & \\
\hline 1394 & $5455=15321$ & PLATE & 1 & PBNFL & $12 \times 12 \times .75$ & MIAMI FLA & TOTAL & 6 & BASE & ALLOY & $\mathbf{P}$ & .0020 & .0000 & \\
\hline 1401 & $5450-4321$ & DI ATE: & 1 & PANEL & $12 \times 12 \times 1$ & MIAMI ELA & TUIAL & 6 & BASE & ALLOY & $\mathbf{P}$ & .0013 & .0006 & \\
\hline 924 & $5456-4321$ & PI ATE & 1 & PANEL & $12 \times 12 \times 2$ & MIAMI FLA & TOTAL & 12 & BASE & ALLOY & I & .0013 & .0010 & \\
\hline 13 月1 & $5456-1.321$ & PLATE & 1 & PANEL & $12 \times 12 \times, 38$ & MIAMI FLA & TOTAL & 12 & BASE & ALLGY & $p$ & .0004 & .0004 & \\
\hline 13 ด & $5856=H 321$ & PLAT: & 1 & PANFI & $12 x+2 x \cdot 5$ & MIAMI FLA & TOJAL & 12 & BASE & ALLOY & & NAA & & \\
\hline 1305 & $5456-4324$ & PI ATE & 1 & $D_{L} N_{F L} L$ & $12 \times 12 \times, 75$ & MIAMI FLA & TOIAI. & 12 & BASE & ALLOY & $\mathbf{P}$ & .0016 & .0020 & \\
\hline $140 ?$ & 545504321 & PIATE & 1 & PANF:L & $12 \times 12 \times 1$ & MIAMT FLA & TOIAL & 12 & VASE & ALLOY & $\mathbf{p}$ & .0012 & .0010 & \\
\hline
\end{tabular}


TABLE I

\begin{tabular}{|c|c|c|c|c|c|c|c|c|c|c|c|c|c|}
\hline \multirow{3}{*}{$\begin{array}{l}\text { IDENT. } \\
\text { NUMER } \\
1407\end{array}$} & \multirow{3}{*}{ 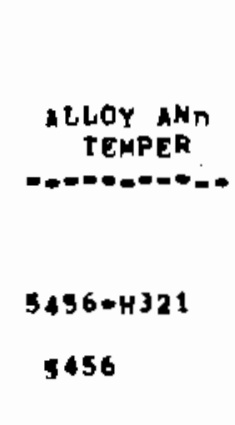 } & \multirow{3}{*}{$\begin{array}{l}\text { PROD. } \\
\text { PLATE }\end{array}$} & \multirow{2}{*}{$\begin{array}{l}\text { SC } \\
\text { PD } \\
\text { ED } \\
\text { CE } \\
--\end{array}$} & \multirow{2}{*}{$\begin{array}{c}\text { PYPE OF } \\
\text { SPFCIMEN } \\
-90-0.00-\end{array}$} & \multirow{2}{*}{$\begin{array}{l}\text { SPECIMEN } \\
\text { SIZE } \\
\text { INCHES } \\
\end{array}$} & \multirow{2}{*}{\multicolumn{2}{|c|}{$\begin{array}{l}\text { DEIAILS OF BPECIMEN } \\
\text { LCCATION }\end{array}$}} & \multirow{2}{*}{$\begin{array}{l}\text { EXPOSED } \\
\text { DEPTH=TT } \\
\text { DE-OH. }\end{array}$} & \multirow{2}{*}{$\ddot{\infty}$} & \multicolumn{4}{|c|}{ RESULTS OF EXAHINATIUN } \\
\hline & & & & & & & & & & LOCATION EXAMINED & TXPE & $\max _{-\infty}$ & MEAN \\
\hline & & & 2 & PANEL & $4 \times 9 \times .375$ & MIAMI & FiA & TOTAL & 12 & $\begin{array}{l}\text { BASE ALLOY } \\
\text { HEATAFTCTED } 20 N E \\
\text { WELD BEAD } \\
\text { KMIFE LINE ATTACK }\end{array}$ & $\begin{array}{l}p \\
p \\
P\end{array}$ & $\begin{array}{l}.0017 \\
.0000 \\
.0014 \\
.0136\end{array}$ & $\begin{array}{l}.0006 \\
.0004\end{array}$ \\
\hline 1400 & $\begin{array}{l}5456=\mathrm{H}^{321} \\
3556\end{array}$ & PLATE & 2 & PANEL & $4 \times 0 \times .375$ & MIAMI & FLA & TOTAL & 12 & $\begin{array}{l}\text { GABE ALLOY } \\
\text { HEATAFTECTED ZONE } \\
\text { WELD BEAD } \\
\text { KNITE LINE ATTACK }\end{array}$ & p & $\begin{array}{l}.0013 \\
.0006 \\
.0068 \\
.0273\end{array}$ & $\begin{array}{l}.0006 \\
.0012\end{array}$ \\
\hline 925 & $5456-H 321$ & PLATE & 1 & PANEL & $12 \times 12 \times 2$ & MIAMI & FLA & TOTAL & 24 & BASE AELOY & $P+51$ & .0237 & .0046 \\
\hline 1392 & $5456=\mathrm{H} 32.1$ & PLATE & 1 & PaNEL & $12 \times, 2 x, 38$ & MIAMI & ris & TOTAL & 24 & BABE ALLOY & 1 & .0142 & .0010 \\
\hline $13 \times 9$ & $5456 \cdot H 321$ & PLATE & 1 & $P_{2} N_{F} L$ & $12 x_{12} \times, 5$ & MIAMI & TLA & TOTAL & 24 & BASE ALLOY & $\mathbf{p}$ & .0030 & .0014 \\
\hline 1396 & $5456-H 321$ & PLATE & 1 & PANEL & $12 \times 12 \times, 75$ & MIAMI & PLA & TOTAL & 24 & BABE ALLOY & $\mathbf{P}$ & .0052 & .0015 \\
\hline 1403 & $5456 \cdot H 321$ & PLATE & 1 & PANEL & $12 \times 12 \times 1$ & MIAKI & rLa & TOTAL & 24 & BABE ALLOY & $p$ & .0127 & .0018 \\
\hline 1410 & $\begin{array}{l}5456-+321 \\
9456\end{array}$ & PIATE & 2 & PAMEL & $4 \times 9 \times, 375$ & MIANI & rLA & TOTAL & 24 & $\begin{array}{l}\text { EASE ALLOY } \\
\text { HEAT AFIECTED ZONE } \\
\text { WELD BCAD } \\
\text { RHIFE LINE ATTACK }\end{array}$ & $\begin{array}{l}\mathbf{P} \\
\mathbf{p} \\
\mathbf{p}\end{array}$ & $\begin{array}{l}.0000 \\
.0013 \\
.0209\end{array}$ & $\begin{array}{l}.0006 \\
.0007 \\
.0007\end{array}$ \\
\hline 1411 & $\begin{array}{l}5456 \cdot H_{321} \\
3556\end{array}$ & PLATE & 2 & PANEL & $4 \times 9 \times, 375$ & MIAMI & PLA & TOTAL & 24 & $\begin{array}{l}\text { BASE ALLOY } \\
\text { HEATAFTECTEO ZONE } \\
\text { WELD BEAD } \\
\text { WIFE LINE ATIACK }\end{array}$ & $\stackrel{p}{P+P}$ & $\begin{array}{l}.0013 \\
.0021 \\
.0246\end{array}$ & $\begin{array}{l}.0006 \\
.0008 \\
.0008\end{array}$ \\
\hline 926 & $5456-H 321$ & PLATE & 1 & PANFL & $12 \times 12 \times 2$ & MIAMI & FLA & TOTAL & 36 & BASE ALLOY & $\mathbf{P}$ & .0023 & .0012 \\
\hline 1383 & $5456-H 321$ & PLATE & 1 & PANFL & $12 \times 12 \times, 3^{8}$ & MLAYI & FLA & TOTAL & 36 & OASE ALLOY & $\mathbf{P}$ & .0006 & .0002 \\
\hline 1390 & $5056 \cdot 4321$ & PLATE & 1 & PAMEL & $12 x_{1} 2 \times .5$ & MIAMI & FLA & TOTAL & 36 & BABE ALLOY & p & .0000 & .0004 \\
\hline 1307 & $5456-4321$ & PLATE & 1 & PANEL & $12 \times 12 x, 75$ & MIAMI & FLA & TOTAL & 36 & BASE ALLOY & $\mathbf{P}$ & .0016 & .0007 \\
\hline 1404 & $5456-4321$ & PIATE & 1 & PANEL & $12 \times 12 \times 1$ & MIAMI & reA & TOTAL & 36 & BASE ALLOY & $\mathbf{p}$ & .0013 & .0007 \\
\hline
\end{tabular}


TABLE I

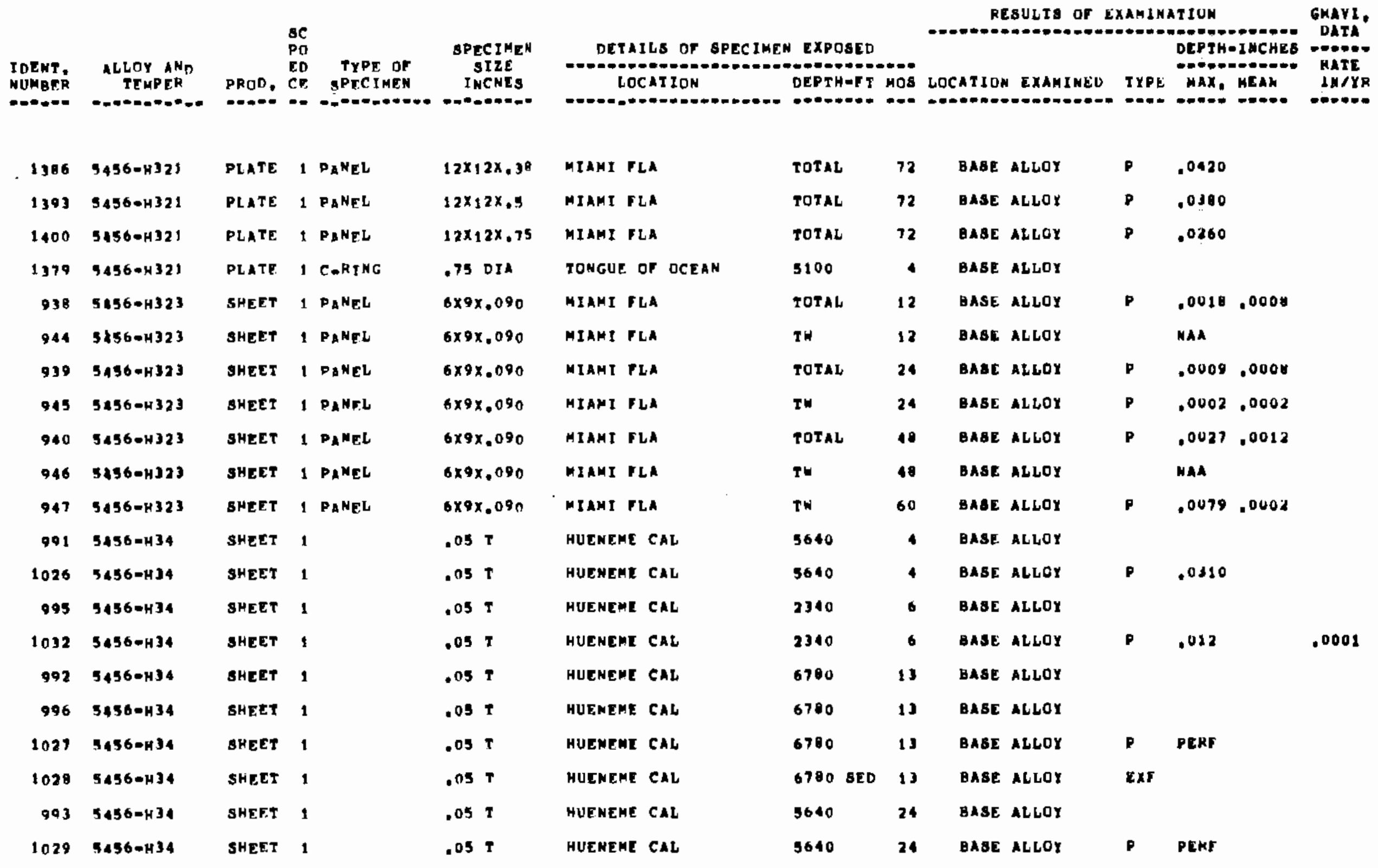


TABLE I

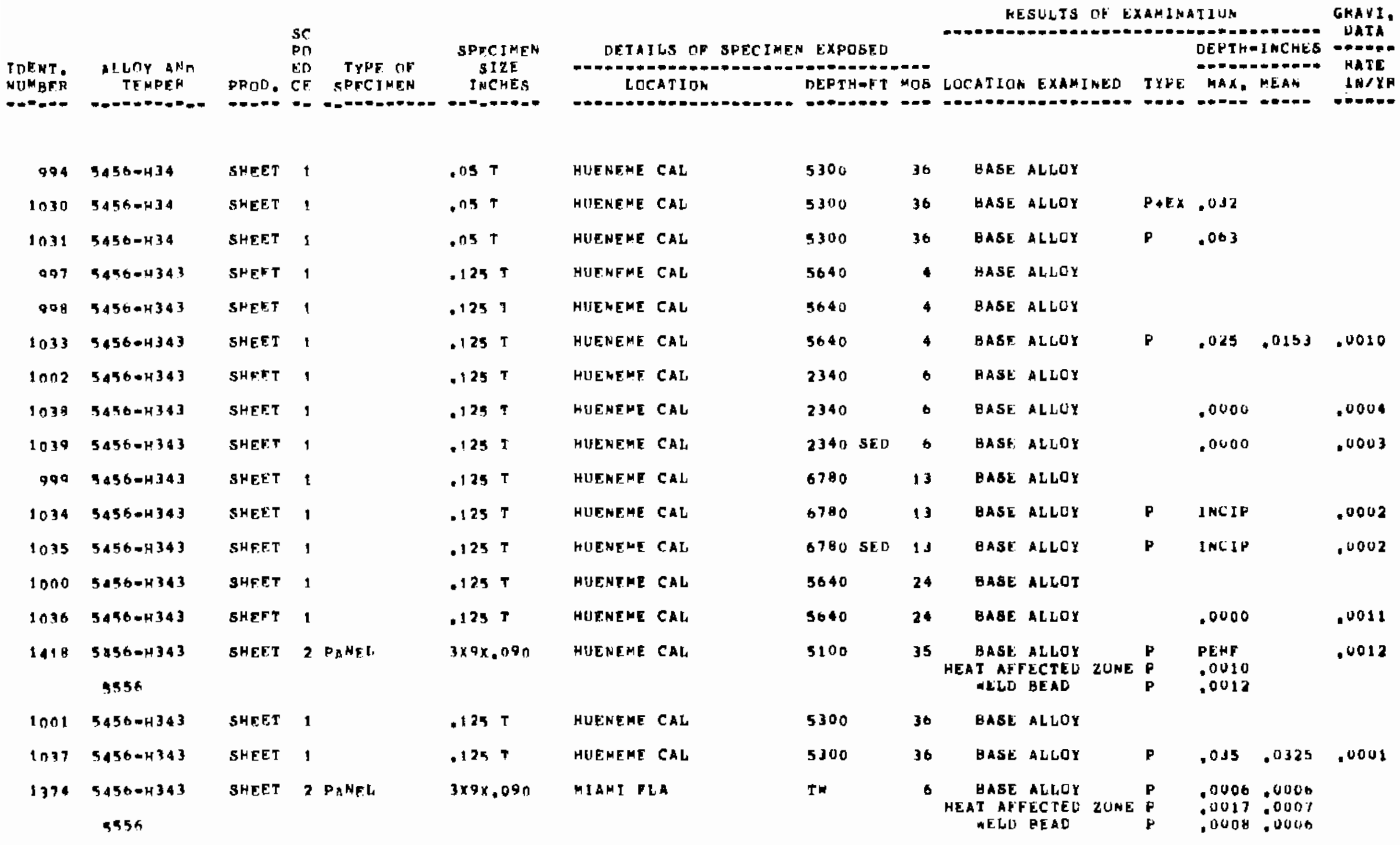


TABLE I

\begin{tabular}{|c|c|c|c|c|c|c|c|c|c|c|c|c|c|c|}
\hline \multirow{3}{*}{$\begin{array}{l}\text { TOENT. } \\
\text { NUMBER } \\
1313\end{array}$} & \multirow{3}{*}{$\begin{array}{l}\text { ALOYY ANn } \\
\text { TEMPER } \\
5456-4343\end{array}$} & \multirow{3}{*}{$\begin{array}{l}\text { PROD, } \\
\text { SHEET }\end{array}$} & \multirow{3}{*}{\begin{tabular}{l} 
8C \\
PO \\
ED \\
CE \\
\hdashline- \\
\end{tabular}} & \multirow[b]{2}{*}{$\begin{array}{c}\text { TYPE OF } \\
\text { SPFCIMEN } \\
-.\end{array}$} & \multirow{3}{*}{$\begin{array}{l}\text { SPECIMEN } \\
\text { BIZE } \\
\text { INCHES } \\
3 \times 9 \times .090\end{array}$} & \multirow{2}{*}{\multicolumn{2}{|c|}{$\begin{array}{l}\text { DETAILE OF SPECIMER } \\
\text { LOCATION } \\
\end{array}$}} & \multirow{2}{*}{$\begin{array}{l}\text { DEPOEED } \\
\text { DEPTHOTT }\end{array}$} & \multirow[b]{2}{*}{ MOS } & \multicolumn{5}{|c|}{ RESULTS OF EXAMINATIUN } \\
\hline & & & & & & & & & & LOCATIOI & N EXAMINED & TYPE & $\operatorname{max.}_{0 \rightarrow 0}$ & $\begin{array}{l}\text { MEAN } \\
-\infty \cdots+\infty\end{array}$ \\
\hline & & & & PANEL & & MIAMI & rLA & TOTAL & 6 & $\begin{array}{r}\text { BASE } \\
\text { HEAT AFI } \\
\text { WELD }\end{array}$ & $\begin{array}{l}\text { ALLOY } \\
\text { FECTED ZUNE } \\
\text { BEAD }\end{array}$ & $\stackrel{p}{p}$ & $\begin{array}{l}.0004 \\
\text { NAA }\end{array}$ & .0084 \\
\hline 941 & $5450-H^{3} 43$ & SHEET & 1 & PAKEL & $6 \times 9 \times .090$ & MIAMI & FLA & TOTAL & 12 & OA6E & ALLOY & $\mathbf{P}$ & .0012 & .0007 \\
\hline 948 & $5456 \cdot H 343$ & BHEET & 11 & PANFL & $6 \times 9 \times .090$ & MIAMI & $r_{L} A$ & TH & 12 & BASE & ALLOX & $\mathbf{p}$ & & .0003 \\
\hline 1424 & $5436 \bullet H^{3} 43$ & SHERT & 11 & PANEL & $4 \times 9 \times, 125$ & MIAMI & PLA & Tw & 12 & GASE & ALLOX & 1 & .0052 & .0011 \\
\hline 1432 & $5456 * H^{3443}$ & SHEET & 11 & PANEL & $4 \times 9 \times .125$ & MIAMI & TLA & TOTAL & 12 & BASI & ALLOY & I & .0000 & .0005 \\
\hline 242 & $5436-\mathrm{H} 343$ & SHEET & 1 & PANEL & $6 \times 9 \times .090$ & MTAMI & FLA & TOTAL & 24 & BASE & ALLOX & $\mathbf{p}$ & .0008 & .0006 \\
\hline 949 & $5456-4343$ & SHEET & 11 & PANEL & $6 \times 9 \times, 090$ & MIAMI & ILA & Tw & 24 & BASE & ALLOX & & MaA & \\
\hline 1425 & $5456-H^{343}$ & GHEET & 1 & PANEL & $4 \times 9 \times .125$ & MIAMI & FLA & TW & 24 & BASE & ALLOX & $I+P$ & .0086 & .0052 \\
\hline 1433 & $5456-4343$ & BHEET & 11 & Pantil & $4 \times 9 \times, 125$ & MIAMI & FLA & TOTAL & 24 & BASE & ALLOY & I & .0075 & .0071 \\
\hline 1426 & $5436-\mathrm{H}^{343}$ & SHEET & 1 & $P_{A} N_{E} L$ & $4 \times 9 \times, 125$ & $\operatorname{mram}$ & Fta & Tw & 36 & BABE & ALLOY & I & .0032 & .0008 \\
\hline 1434 & $5456 \cdot \mathrm{H} 343$ & SHEET & 1 & PANEL & $4 \times 9 \times .125$ & MIAMI & FLA & TOTAL & 36 & BASE & ALCOX & 1 & .0021 & .0007 \\
\hline 943 & $5456-\mathrm{H} 343$ & SHEET & 1 & PANEL & $6 \times 9 \times .090$ & MIAMI & FLA & TOTAL & 40 & BASE & ALLOY & $P$ & .0010 & .0006 \\
\hline 950 & $5456-H 343$ & SHEET & 91 & PANEL & $6 \times 9 \times .090$ & MZAMI & FLA & $T w$ & 48 & BA8E & ACLOY & & waA & \\
\hline 1427 & $5456-H 343$ & SHEET & 11 & $P_{A} N_{F L}$ & $4 \times 9 \times .125$ & MIAMI & FLA & $T *$ & 40 & BASE & ALLOX & I & .0024 & .0000 \\
\hline 1435 & $5456 \cdot \mathrm{H} 343$ & SHEET & 1 & PANEL & $4 \times 9 \times, 125$ & MIAMI & FLA & TOTAL & 48 & BABE & ALLOY & 1 & .0040 & .0015 \\
\hline 951 & $3456-H 343$ & SHEET & 1 & PANEL & $6 \times 9 \times .090$ & MIAMI & FLA & $T W$ & 60 & BASE & ALLOY & $\mathbf{P}$ & .0058 & .0033 \\
\hline 1429 & $3456-1343$ & SHECT & 11 & PANEL & $4 \times 9 \times, 125$ & MIAHI & rLA & TH & 60 & BASE & ALLOY & I & .0030 & .0008 \\
\hline 1436 & $5456-H 343$ & SHECt & 1 & PANFL & $4 \times 9 \times, 125$ & MIAMI & FLA & TOTAL & 60 & BABE & ALLOY & $I+P$ & .0250 & .0011 \\
\hline 1429 & $5456=\mathrm{H} 343$ & SHEET & 1 & PANEL & $4 \times 9 x, 125$ & MIAMI & FLA & TH & 72 & BASE & ALLOX & I & .0112 & .0036 \\
\hline 1437 & $5456 \cdot H 343$ & SHEET & 1 & PANTL & $4 \times 9 \times, 125$ & MIAMI & FEA & TOTAL & 72 & BABE & ALLOY & I & .0079 & .0029 \\
\hline
\end{tabular}


TABLE I

\begin{tabular}{|c|c|c|c|c|c|c|c|c|c|c|c|c|c|c|}
\hline \begin{tabular}{l} 
FDENT, \\
NUMBER \\
\hdashline$-9 .-S$
\end{tabular} & $\begin{array}{l}\text { ALLOY AND } \\
\text { TEMPEA } \\
-\end{array}$ & PRחD: & $\begin{array}{l}8 c \\
P D \\
E D \\
C E \\
\therefore\end{array}$ & 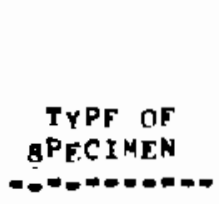 & 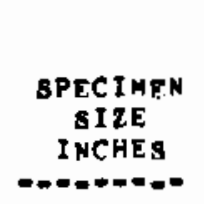 & $\begin{array}{l}\text { DETALLS OF OPECIMEN } \\
\text { LOCATIOH }\end{array}$ & $\begin{array}{l}\text { DEXPOSED } \\
\text { DEPTHEFT } \\
\text { DEATH }\end{array}$ & MOS & LOCATIOH & EsULis of E & TYPE & $\begin{array}{l}\text { ATIUN } \\
\text { DEFTH: } \\
\text { maX. } \\
\text { MET: }\end{array}$ & 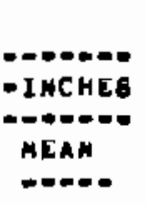 & 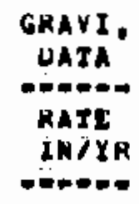 \\
\hline 919 & $5457 \cdot H_{34}$ & SHEET & 1 & PANFL & $4 \times 17 \times .04$ & HARBOA ISLAND NC & TOTAL & 24 & EASE & ALLOY & $\mathbf{P}$ & .0117 & .0002 & .0001 \\
\hline 917 & $5457=\mathrm{H} 34$ & SHEET & 1 & PANEL & $4 \times 12 \times, 04$ & HAPBOR ISLLAND NC & $T w$ & 60 & BASE & ALLOY & $\mathbf{P}$ & .0046 & .0001 & .0001 \\
\hline 920 & $5457-\mathrm{H}^{34}$ & SHEET & 1 & $P_{\text {A NEL }}$ & $4 \times 12 \times .04$ & HARBOR ISLAND NC & TOTAL & 60 & BASE & ALLOX & $P$ & .0037 & .0002 & .0001 \\
\hline 821 & $6051-74$ & PLATE & 1 & PANFL & $12 \times 12 \times, 25$ & ESOUIMALT BC & TOTAL & 12 & GASE & ALLOY & $P$ & .010 & & \\
\hline 825 & $6051-T 4$ & PLATE & 1 & PANEL & $12 \times 12 \times, 25$ & ESOUIMALT BC & TOTAL & 12 & BASE & ALLOY & $\mathbf{p}$ & .025 & & \\
\hline 845 & $6051=T^{4}$ & AHGLE & 1 & ANGLE & $2 \times 2 \times 36$ & ESOUIMALT BC & TOTAL & 12 & BABE & ALLOY & $\mathbf{P}$ & .035 & & \\
\hline 849 & $6051-74$ & ANGLE & 1 & $A_{N} G_{L} E$ & $2 \times 2 \times 36$ & ESOUIMALT BC & TOTAL & 12 & BASE & ALLOY & $\mathbf{p}$ & .006 & & \\
\hline 892 & $\ln 51 \cdot T 4$ & PLATE & 1 & PANEL & $12 \times, 2 \times .25$ & ESOUIMALT BC & TOTAL & 24 & BABE & ALLOY & $\mathbf{P}$ & .065 & & \\
\hline B26 & $6 n 51-T 4$ & PLATE & 1 & PANEL & $12 \times 12 \times, 25$ & ESOUIMALT BC & TOTAL & 24 & BASE & ALLOY & $\mathbf{p}$ & .047 & & \\
\hline A46 & $6051 \cdot 74$ & ANGLE & 1 & ANGLE & $2 \times 2 \times 36$ & csouIMaLT BC & TOTAL & 24 & BABE & ALLOY & p & .023 & & \\
\hline 85n & $\operatorname{sn} 51-54$ & ANGLE & 1 & $A N G L E$ & $2 \times 2 \times 36$ & EBOUIMALT BC & TOTAL & 24 & BASE & ALLOY & $P$ & .005 & & \\
\hline 923 & $6051-74$ & PLATE & 1 & PANFL & $12 x_{1} 2 x_{0} 25$ & ESOUIMALT BC & TOTAL & 60 & BASE & ALLOY & $\mathbf{P}$ & .051 & & \\
\hline 027 & $6051-54$ & Plate & 1 & PANEL & $12 \times 12 \times, 25$ & ESOUIMALT BC & TOTAL & 60 & BABE & ALLOY & $\mathbf{P}$ & .109 & & \\
\hline 947 & $6051-74$ & ANGLE & 1 & ANGLE & $2 \times 2 \times 36$ & ESOUIMALT BC & TOTAL & 60 & BASE & ALLOY & $\mathbf{p}$ & .005 & & \\
\hline 851 & $6051-T^{4}$ & ANGLE & 1 & $A N G E_{1} E$ & $2 \times 2 \times 36$ & ESOUIMALT BC & TOZAL & 60 & BASE & ALLOY & $\mathbf{P}$ & .000 & & \\
\hline 824 & $6051 \cdot 44$ & PLate & 1 & PANEL & $12 \times 12 \times .25$ & ESOUIMALT BC & TOTAL & 120 & BASE & ALLOY & $\mathbf{P}$ & .037 & & \\
\hline 828 & $6051-74$ & PLATE & 1 & PANFL & $12 \times 12 \times .25$ & ESOUIMALT BC & TOTAL & 120 & BASE & ALLOY & $\mathbf{p}$ & .170 & & \\
\hline 848 & $6051-74$ & ANGLE & 1 & ANGLE & $2 \times 2 \times 36$ & EGOUIMALT BC & TOTAL & 120 & BASE & ALLOY & $\mathbf{P}$ & .072 & & \\
\hline 852 & $6051-T^{4}$ & ANGLE & 1 & ANGLE & $2 \times 2 \times 36$ & ESOUIMALT BC & TUTAL & 120 & BASE & ALLOY & $\mathbf{p}$ & .115 & & \\
\hline 837 & $6051=+4$ & ANGLE & 1 & ANGLE & $2 \times 2 \times 36$ & HALIFAX NS & TOTAL & 12 & BASE & ALLOY & $\mathbf{P}$ & .027 & & \\
\hline BAs & $6051-T^{4}$ & ANGLE & 1 & ANG $G_{L} E$ & $2 \times 2 \times 36$ & HALIFAX NS & TOTAL & 12 & BASE & ALLOX & $\mathbf{P}$ & .034 & & \\
\hline
\end{tabular}


TAELE I

\begin{tabular}{|c|c|c|c|c|c|c|c|c|c|c|c|c|c|c|c|}
\hline $\begin{array}{l}\text { THENT: } \\
\text { NIMAEP }\end{array}$ & $\begin{array}{c}\text { ALILCY AXin } \\
\text { TFMFFh }\end{array}$ & FFHD. & $\begin{array}{l}\text { Sr } \\
\text { Pri } \\
\text { Fr } \\
C F\end{array}$ & $\begin{array}{l}\text { TYPF חF } \\
\text { SPFCTMEN }\end{array}$ & $\begin{array}{l}\text { SPFCIMEN } \\
\text { SIZE } \\
\text { INCHES }\end{array}$ & $\begin{array}{r}\text { DETAL } \\
\text { LOC }\end{array}$ & $\begin{array}{l}\text { ILS TE } \\
\text { CATION }\end{array}$ & SHECIMEN & $\begin{array}{l}\text { VXPOSED } \\
\text { DEPTHEFT }\end{array}$ & Mns & Kt & LSUL, IS UF KX & CAMINA & $\begin{array}{l}\text { TIUN } \\
\text { DEPTHEINCHES } \\
\text { MAX, MEAN }\end{array}$ & $\begin{array}{l}\text { GNAVI, } \\
\text { DATA } \\
\text { KATE } \\
\text { INIYH }\end{array}$ \\
\hline$+\infty-\infty$ & $-\infty-\infty-0-0-0$ & $-\infty-\infty$ & $=$ & 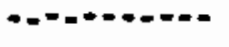 & $=+-\infty+\infty+\infty$ & $\theta-\infty+\infty$ & $-\infty-\infty-\infty$ & 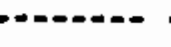 & $--+-\infty-\infty$ & $m$ & n-m-nen & 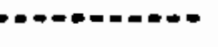 & $+\infty-\infty$ & $\infty-\infty+\infty+\infty$ & $-\infty+\infty$ \\
\hline 907 & $6051=74$ & PIATE & 1 & DAPIEL & $12 \times 12 \times .25$ & HALTFAX & Ns & & TOIAL & 12 & BASE & ALLOY & & NAA & \\
\hline 911 & $5051-T^{4}$ & PI ATE: & 1 & $P_{Q} N E L$ & $12 \times 12 \times, 25$ & HALIFAX & NS & & TOIAL & 12 & BASE & ALLOY & $\mathbf{P}$ & .0030 & \\
\hline A I B & $6051-T^{4}$ & ANGTE & 1 & 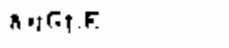 & $2 \times 2 \times 36$ & HALIFAX & NS & & $T O T A L$ & 24 & BASE & ALLCY & $p$ & .027 & \\
\hline 942 & $\ln 51=-74$ & AR:CILE. & 1 & A SeT $F$ & $3 \times 2 \times 36$ & HALIFAX & its & & TUTAL & 24 & BASE & ALLOY & $\mathbf{p}$ & .005 & \\
\hline$Q \cap B$ & $6051=T^{4}$ & PIATE & 1 & 0.451 & $12 \times 92 \times .25$ & HALIFAX & NS & & TOTAL & 24 & RASE & ALLCY & $p$ & .010 & \\
\hline 912 & 6n51-T4 & PIATF & 1 & $D_{\triangle N}=L$ & $12 \times 12 \times \cdot 25$ & HAL.IFAX & NS & & TOTAL & 24 & AASE & ALLOY & & NAA & \\
\hline 939 & $\operatorname{Rn} 51-T 4$ & ANGIE & 1 & $A x \cdot C \mid F$ & $2 \times 2 \times 36$ & HALIFAX & NS & & TOIAL & 60 & EASE & ALLOY & $\mathbf{p}$ & .014 & \\
\hline ค 43 & $6051-74$ & $A N C, L F$ & 1 & $A \times G t E$ & $2 \times 2 \times 36$ & HALIFAX & NS & & TUIAL & 60 & BASE & AI LOY & $\mathbf{P}$ & .030 & \\
\hline 909 & $\operatorname{nn} 51=74$ & PIATE & 1 & DaNEL & $12 x, 2 x \cdot 25$ & HALIFAX & NS & & TOTAL & 60 & GASE & ALLEY & & NAA & \\
\hline 913 & $6051-74$ & PIATF & 1 & DANFL & $12 \times 12 \times .25$ & HALIFAX & NS & & TOTAL & 60 & BASE & ALLOY & $\boldsymbol{v}$ & .0000 & \\
\hline 840 & BO5 $1=T^{4}$ & ANGLE & 1 & ancit $\bar{F}$ & $2 \times 2 \times 36$ & HALIFAX & NE & & TUTAL & 120 & BASE & ALLCY & $\mathbf{P}$ & .012 & \\
\hline 944 & $6051-74$ & ANGLE & 1 & $A=C^{2}$ & $2 \times 2 \times 30$ & HALIFAX & NS & & TUTAL. & 120 & HASE & ALLUY & $\mathbf{p}$ & .014 & \\
\hline 910 & $6 \cap 51-T 4$ & PIATE & 1 & DANFL & $12 \times 12 \times .25$ & HALIFAX & NS & & TITAL & 120 & BASE. & ALLOY & $\mathbf{P}$ & .002 & \\
\hline 914 & $\operatorname{sn} 51=+4$ & PI.ATE & 1 & D $\triangle N F I$ & $12 \times 12 \times .25$ & HALIFAX & NE & & TOTAL & 120 & EASE & ALLOY & $\boldsymbol{p}$ & .045 & \\
\hline$R \geq 9$ & $\ln 51=+4$ & $A N G, 1, F$ & 1 & axGIE & $2 \times 2 \times 36$ & HARGOH & ISLAND & NC: & TOTAL & 12 & DASE & ALLDY & & NAA & \\
\hline 93.3 & $\ln 51-T^{4}$ & ANCIT: & 1 & ANGP $E$ & $2 \times 2 \times 36$ & HAREOR & ISLXND & NC & TOIAL & 12 & BASE & ALLOY & $\mathbf{P}$ & . OS & \\
\hline 909 & $6 \cap 51-T 4$ & PLATE & 1 & DANEL & $12 \times 12 \times .25$ & HARROA & ISLAND & $\mathrm{NC}$ & TOTAL & 12 & BASE & ALLOY & & NAA & \\
\hline $9 \cap 3$ & $6051-74$ & DIATH: & 1 & FANEL & $12 x_{12} \times 25$ & HARPQR & ISLAND & $N C$ & TOTAL & 12 & BASE & ALLOY & $\mathbf{p}$ & .016 & \\
\hline 930 & GO5 I- TA & ANGI,F & 1 & $A x \in t E$ & $2 \times 2 \times 36$ & HARRDP & ISLAND & NC & TOTAL & 24 & HASE & ALLOY & & NAA & \\
\hline A $\times 4$ & $6051=+4$ & ARTELF & 1 & Ancitf $F$ & $2 \times 2 \times 36$ & HAKKIDE & ISLARD & HC & TOIAL & 24 & HASE & $A L L C I$ & $\mathbf{P}$ & $.005 u$ & \\
\hline 900 & $\operatorname{Rn} 51=T 4$ & Dith. & 1 & $\mathrm{P}_{\mathrm{N}} \mathrm{NF}$ & $17 x+2 x .25$ & HAKAOK & ISLAND & $\mathrm{NC}$ & TOIAL & 24 & BASE & ALLOX & & $N A A$ & \\
\hline
\end{tabular}


TABLE I

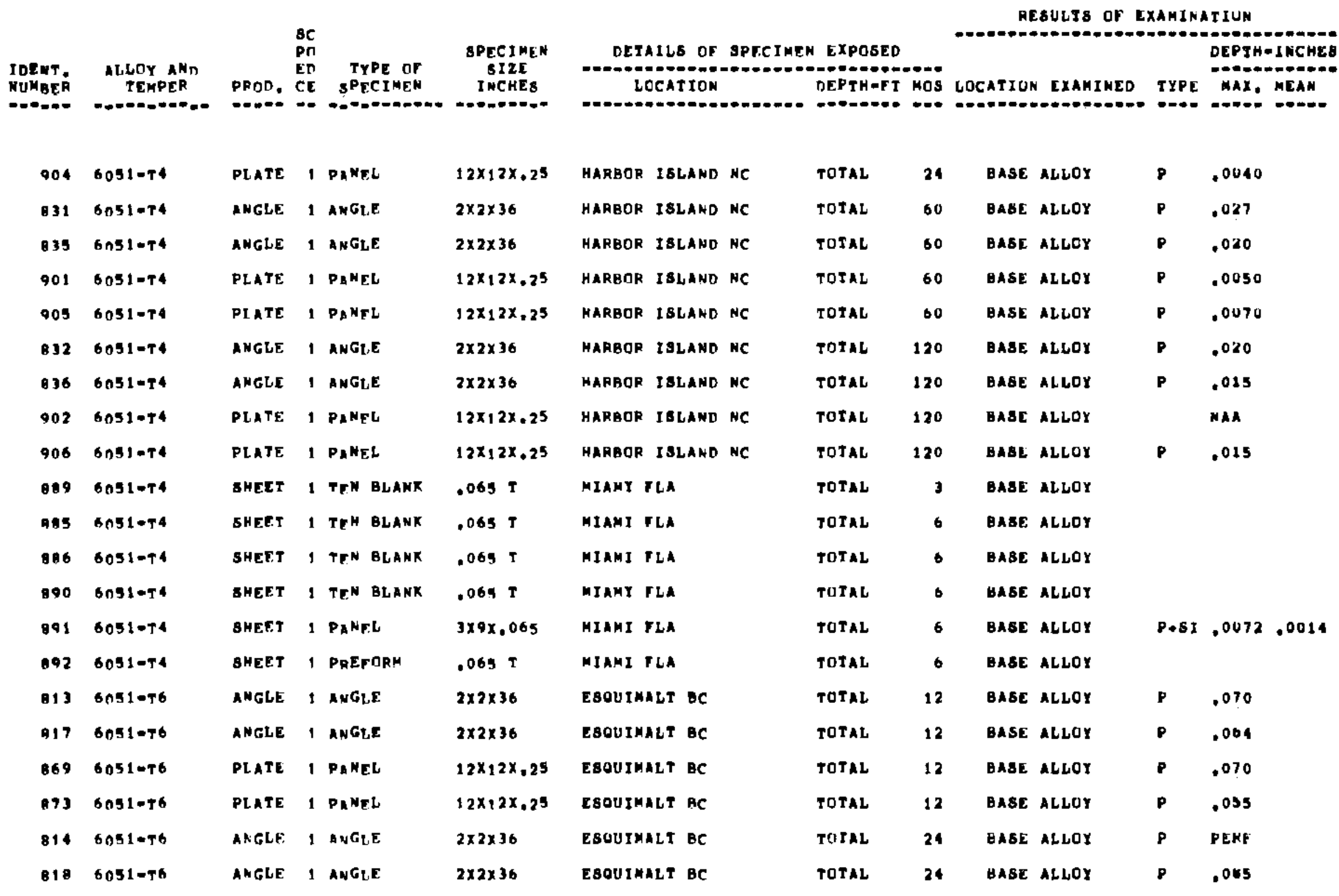

MAYI. DATA - R-mer Mr 
TAELE 1

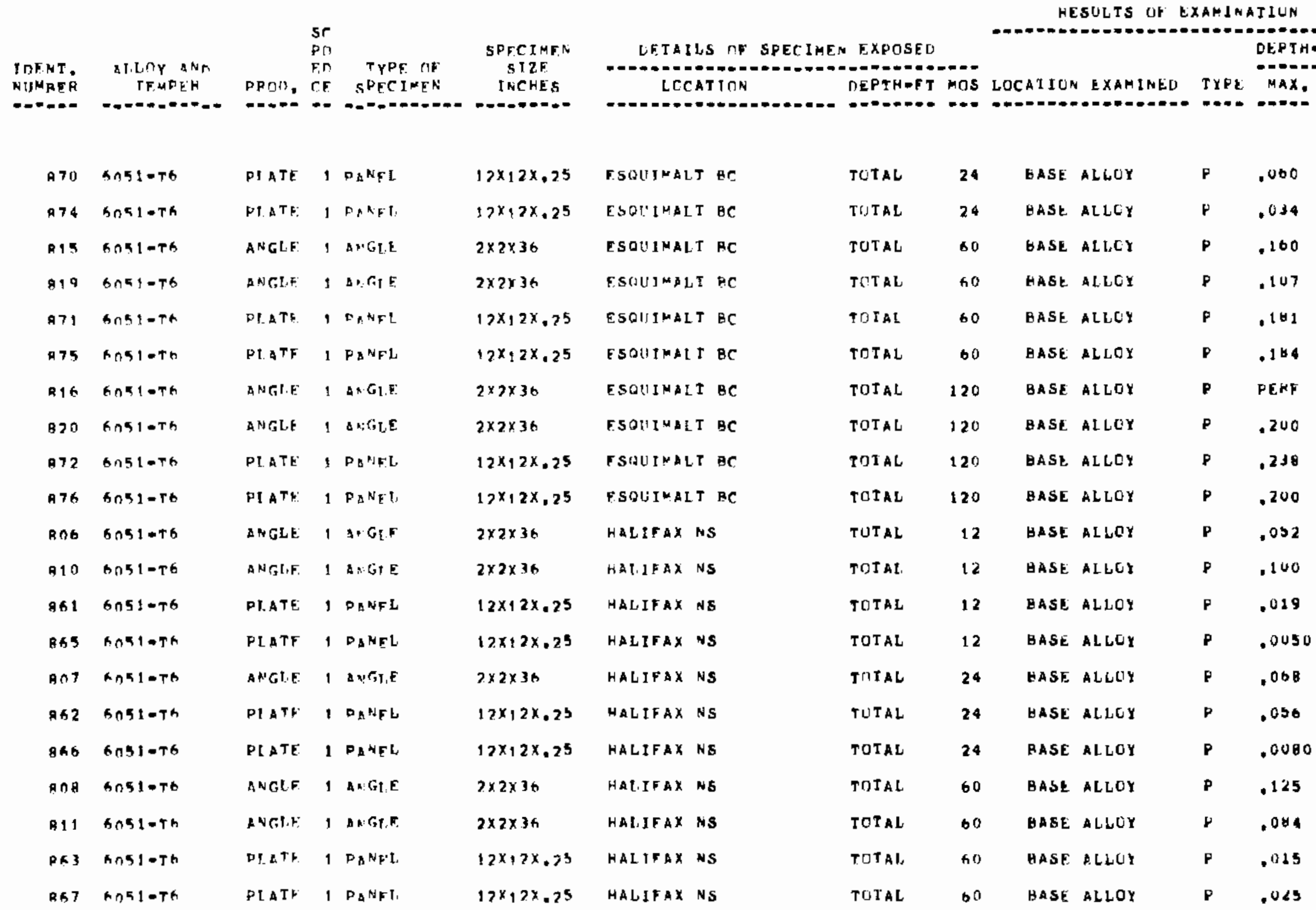


TABLE I

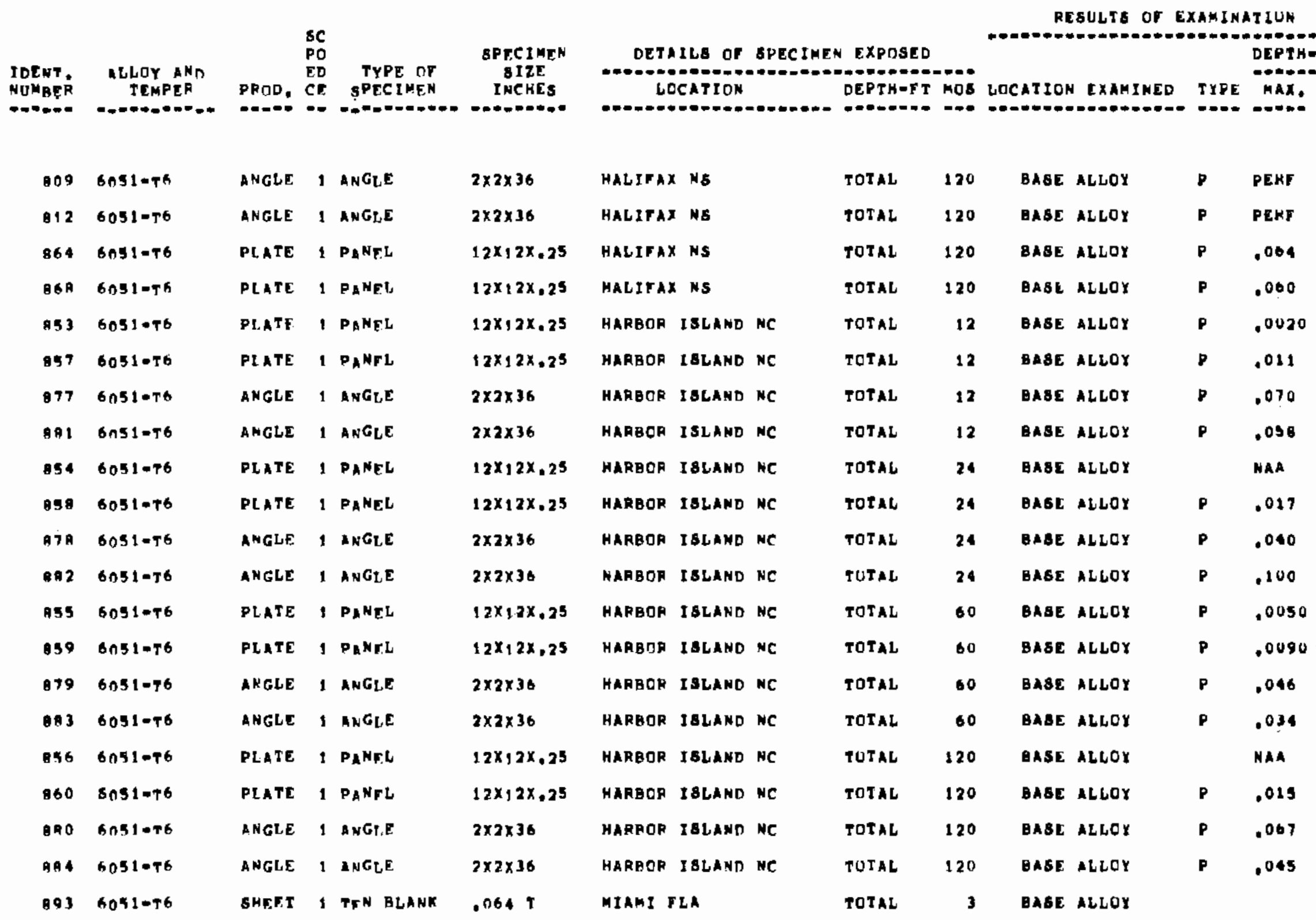


TAPLE I

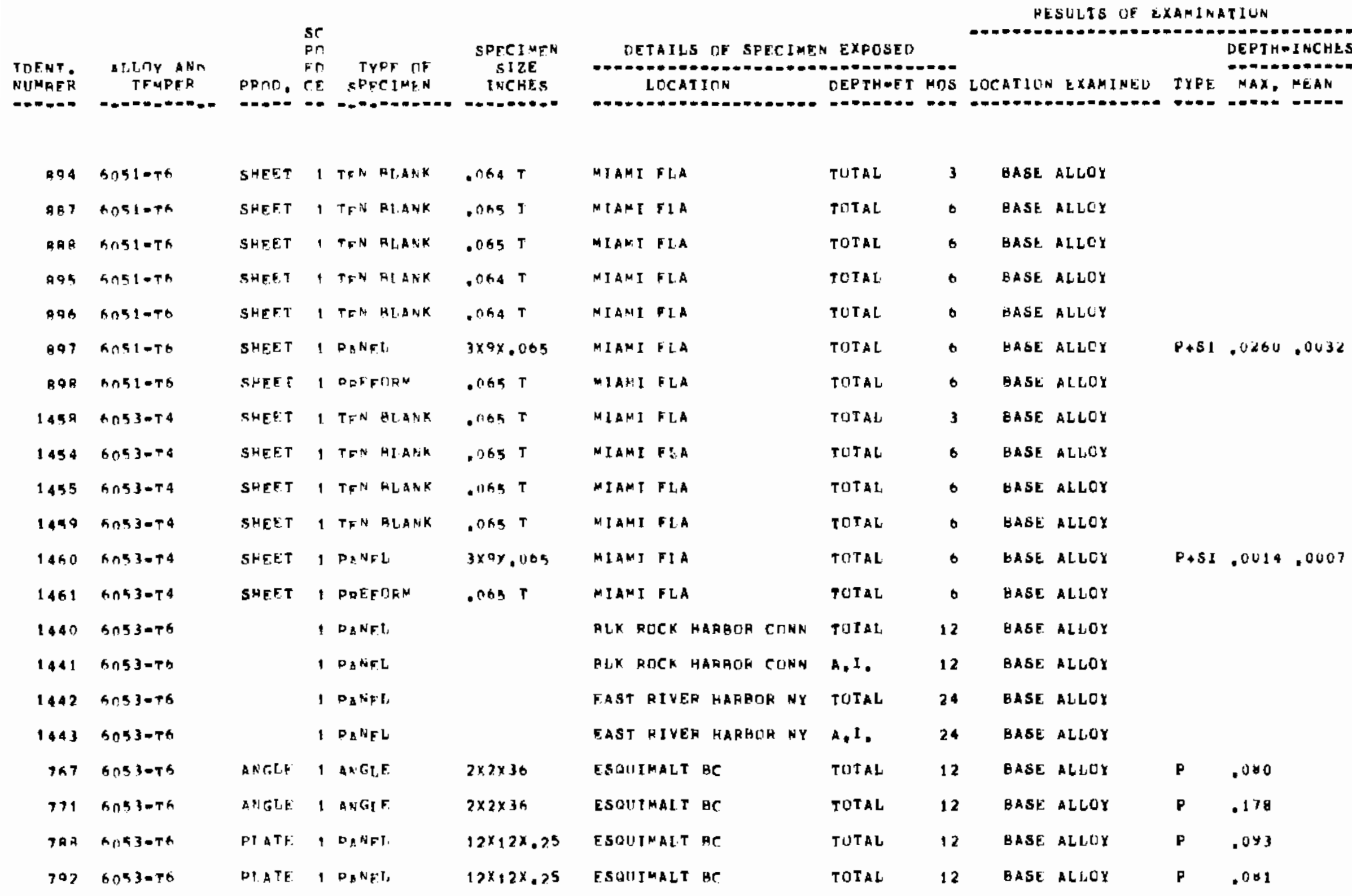


TABLE I

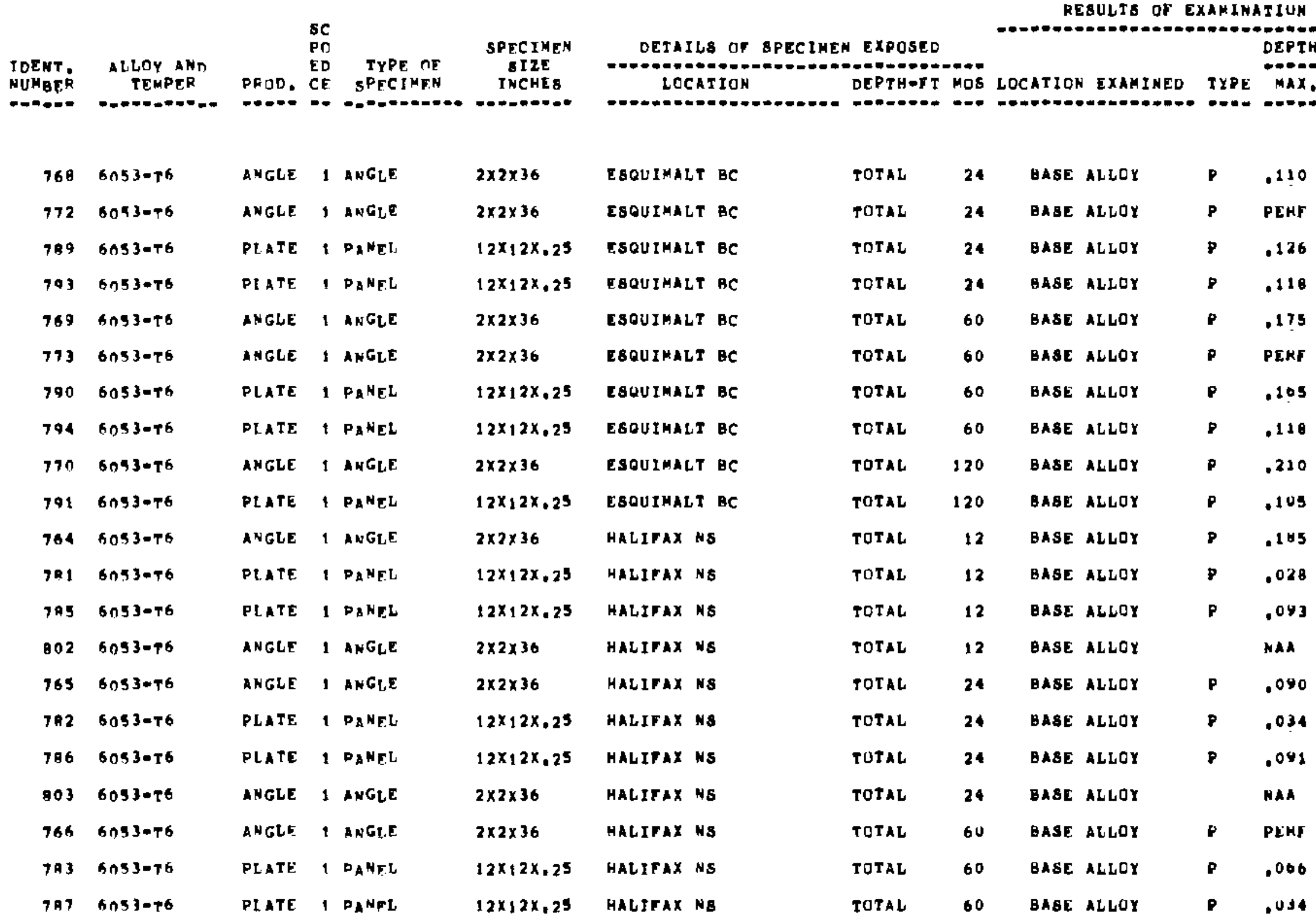


TABLE I

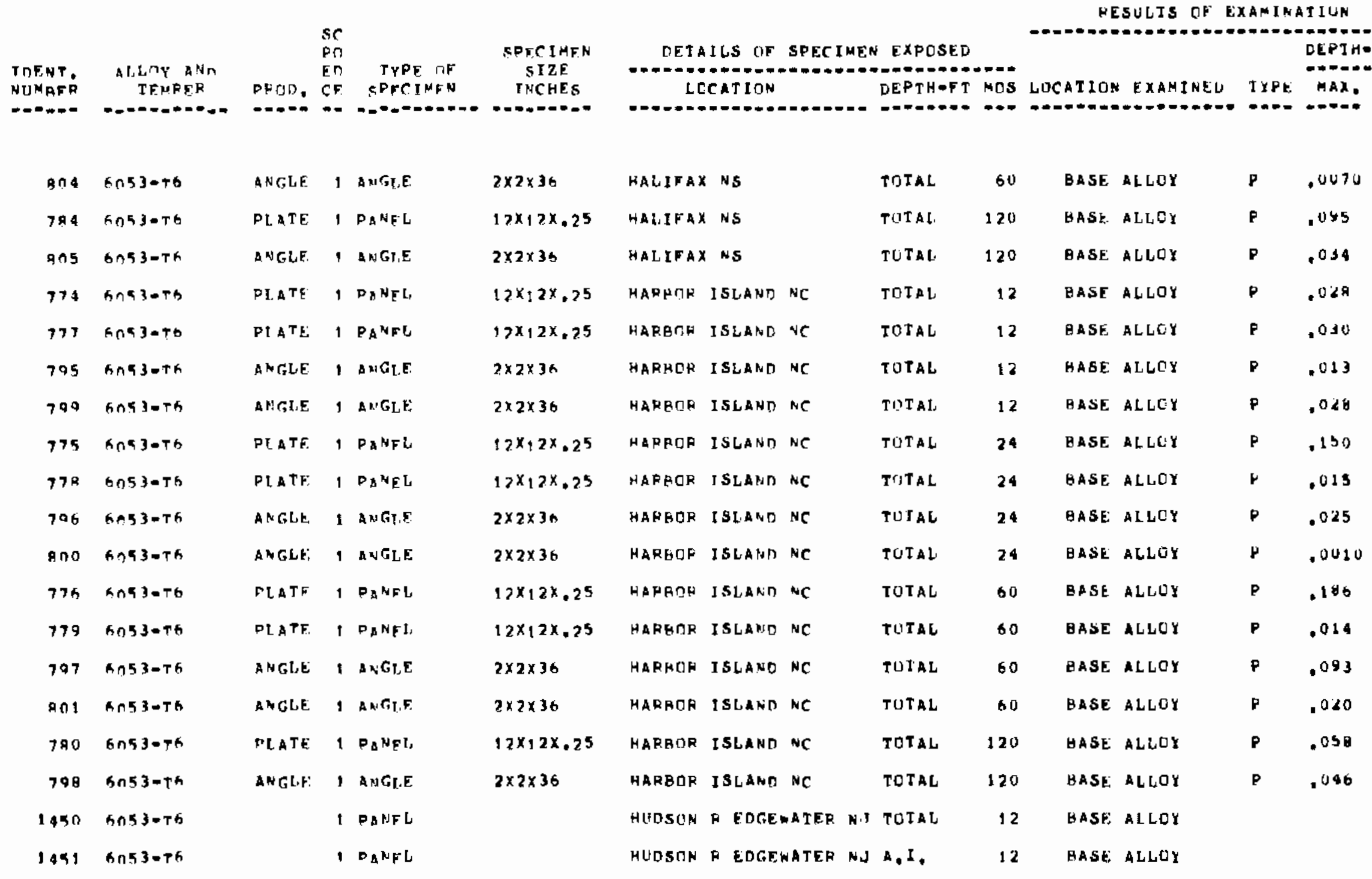


TABLE I

\begin{tabular}{|c|c|c|c|c|c|c|c|c|c|c|c|c|c|c|}
\hline $\begin{array}{l}\text { TDENT: } \\
\text { NUABER } \\
=-\infty\end{array}$ & 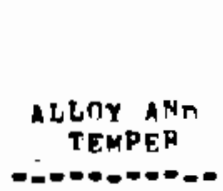 & PROD, & $\begin{array}{l}\text { SC } \\
\text { PO } \\
\text { ED } \\
\text { CE } \\
-.\end{array}$ & $\begin{array}{r}\text { TYPE OF } \\
\text { SPECIMEN } \\
\text { - }\end{array}$ & $\begin{array}{l}\text { SPECI MEN } \\
\text { SIZE } \\
\text { INCHES } \\
\text { INC- }\end{array}$ & $\begin{array}{l}\text { DETAILS OF BPECIMEN } \\
\text { LOCATION }\end{array}$ & $\begin{array}{l}\text { EXPOSED } \\
\text { DEPTH-FT } \\
0=-0-0=-\pi\end{array}$ & $\operatorname{mos}$ & LOCAIION & ESULTE OF E & XAMINI & 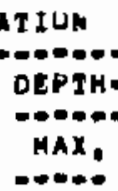 & 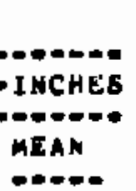 & 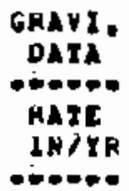 \\
\hline 2433 & $\begin{array}{c}6053-76 \\
4043\end{array}$ & PLATE & 2 & PANEL & $.25 \mathrm{~T}$ & KURE BEACH NC & TOTAL & 24 & $\begin{array}{l}\text { GASE } \\
\text { WELD }\end{array}$ & $\begin{array}{l}\text { ALLOY } \\
\text { BEAD }\end{array}$ & & $\begin{array}{l}.0050 \\
.0042\end{array}$ & $\begin{array}{r}.0012 \\
.0021\end{array}$ & \\
\hline 1452 & $6053-76$ & PLATF. & 1 & RTVETED & $.25 \mathrm{t}$ & KUAE BEACH NC & TOTAL & 72 & BASE & ALLOY & & .0014 & .0005 & \\
\hline 1462 & $6053-\mathrm{T} 6$ & SHEET & 1 & TFN BLANK & $.065 \mathrm{~T}$ & MIAMI FLA & TOTAL & 3 & BASE & ALLOY & & & & \\
\hline 1453 & $6 \cap 53-T 6$ & SHEET & 1 & TFN BLANK & $.065 \mathrm{~T}$ & MIAMI ILA & TOTAL & 3 & BASE & ALLOY & & & & \\
\hline 1456 & $60 \$ 3=T 6$ & SHEET & $\mathfrak{1}$ & TFN BLANK & $.065 \mathrm{~T}$ & MIAMI FLA & TOTAL & 0 & BASL & ALLOY & & & & \\
\hline 1457 & $6053-76$ & SHEET & 1 & TFN BLANK & $.065 \mathrm{~T}$ & MIAMI TLA & TOTAL & 6 & BABE & ALLOY & & & & \\
\hline 1464 & $6053 * 78$ & SHEET & 1 & TEN BLANR & .0651 & MIAMI FLA & TOTAL & 6 & BASE & ALLOY & & & & \\
\hline 1465 & $6053-76$ & SHEET & 1 & TFN BLANK & $.065 \mathrm{~T}$ & MIAHI ILA & TOTAL & 6 & BASE & ALLOY & & & & \\
\hline 1456 & $6053=\pi 6$ & SHEET & 1 & PaNEL & $3 \times 9 \times .065$ & MIAMI ILA & TOTAL & 6 & BASE & ALLOY & $P+E I$ & .0043 & .0007 & \\
\hline 1467 & $6053=76$ & SHEET & 1 & PAFTORP & $.065 \mathrm{~T}$ & MIAMI TLA & TOTAL & 6 & GASE & ALLOY & & & & \\
\hline 1446 & $6053=T 6$ & & 1 & PANFL & & MUBIEE ALA & TOTAL & 24 & BASE & ALLOX & & & & \\
\hline 1447 & $6053-76$ & & 1 & PANFL & & MOBILE ALA & A.I. & 24 & BASE & ALLOY & & & & \\
\hline 1438 & $6053=76$ & & 1 & PANFL & & MONTREAL OUE & TOTAL & 12 & BASE & ALLOY & & & & \\
\hline 1439 & $6053 \cdot T^{6}$ & & 1 & DANEL & & MONTREAL OUE & A.I. & 12 & BAst & ALLOY & & & & \\
\hline 1448 & $6053 \cdot T^{6}$ & & 1 & PANEL & & NEW ORLEANS LA & TOTAL & 24 & BASE & ALLOY & & & & \\
\hline 1449 & $6053-76$ & & 1 & PANEL & & NEW DRLEANG LA & A.I. & 24 & BABE & ALLOY & & & & \\
\hline 1444 & $6053=76$ & & 1 & PANEL & & NORFOLK VA & TOTAL & 12 & BASE & ALLOY & & & & \\
\hline 1445 & $6053=76$ & & 1 & DANFL & & MORFDLX VA & $A, I$, & 24 & BASE & ALLOY & & & & \\
\hline 1470 & K061- & SHEFT & 1 & & $.037 \mathrm{~T}$ & HUENEME CAL & 5 & $b$ & BASE & ALLOY & $\mathbf{p}$ & .0050 & & .0012 \\
\hline 1471 & $6061=$ & SHEET & 1 & & $.037 \mathrm{~T}$ & HUENEME CAL & 5 & 12 & BABE & ALLOY & $p$ & .011 & & .0000 \\
\hline
\end{tabular}


TABLE I

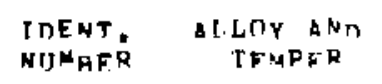
$0-\infty=$ $-0-0$

\begin{tabular}{|c|c|}
\hline 468 & $n \cap \times 1=$ \\
\hline $14 \times 9$ & Snal $=$ \\
\hline 1540 & GnG $1=t$ \\
\hline 1541 & GOKI-T \\
\hline 1542 & GDAl-T \\
\hline 1543 & ank $1=T$ \\
\hline 1544 & GOALA \\
\hline 1545 & $G \cap A 1$ \\
\hline 637 & $G \cap 61-T$ \\
\hline 639 & $\tan \alpha 1=$ \\
\hline 639 & $\tan$ \\
\hline 640 & SOS: $=T$ \\
\hline 633 & $6 \cap 61-7$ \\
\hline 665 & Goh 1 \\
\hline 634 & AOB $1=T$ \\
\hline $66 x$ & 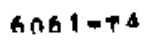 \\
\hline 635 & $6061-T 4$ \\
\hline 647 & SOB $1=44$ \\
\hline 636 & KDAt $=T$ \\
\hline $66 R$ & $\operatorname{son} 1=7$ \\
\hline 629 & 600 \\
\hline
\end{tabular}

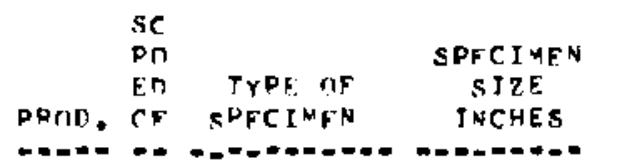

SHFET

SHEFT 1

PIATE I PANEL

PIATE 1 PANFI

PLATE I PANFE

PEATE I PANFL

PIATE, DANFL

HIATE I PANFL

PLATE 1 PANFL

PLATE, DANFt,

PLATE 1 PANFL

PIATE 1 PANFL

ANGLE, ANGLE

PLATE I PANFL

ANGLE I AMGTE

PIATE 1 PANFL

ANGLE I ANGIE

PLATE, PANFL

ANCLE, I ANGTE.

PIATE 1 PANFL

Aneter 1 angite:

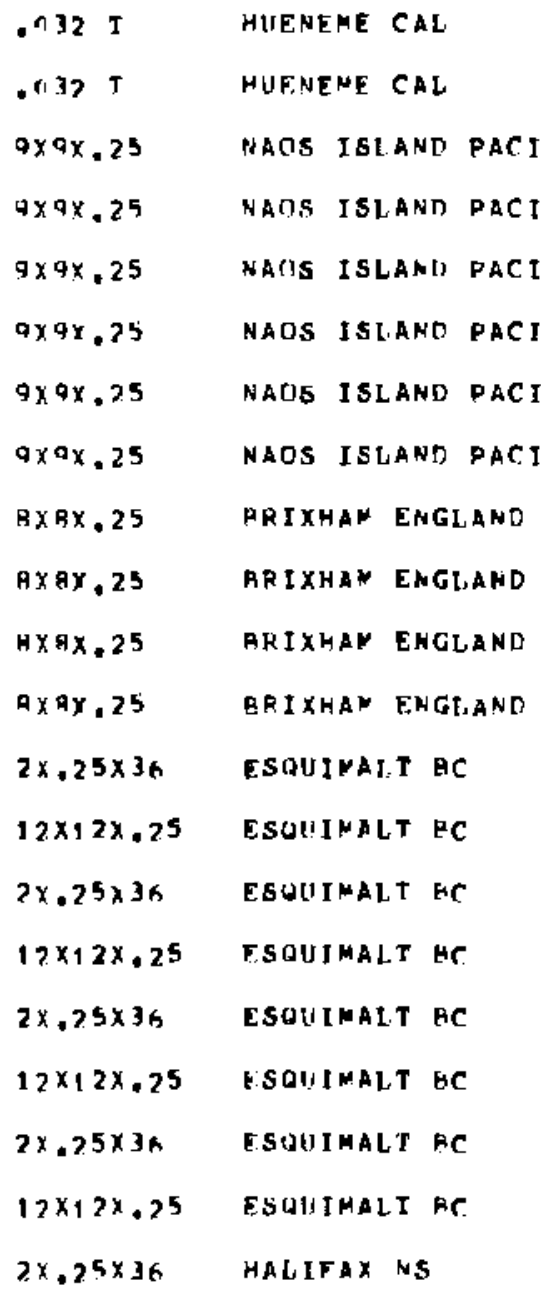

RESULTS UF EXAMINATIUN GKAYI. DEPIHAIHCHEB ..... Max. MEATE KATE DETAILS DP SPECIMEN EXPOSED LCCATION DEPTHFT MIS LOCATIUN EXAHINED TYPE MAX. MEAN IN/YR

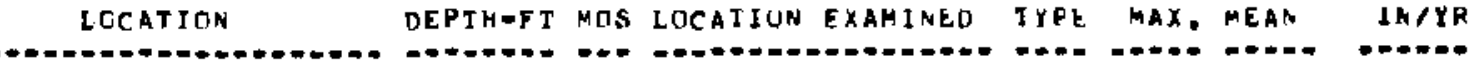

\begin{tabular}{|c|c|c|c|c|c|c|c|}
\hline $237 n$ & 13 & BASE & ALLDY & & NAA & & .0022 \\
\hline 2370 SFD & 13 & AASE: & ALLCY & & NAA & & .0007 \\
\hline 13 & 12 & BASE & ALLOY & $\boldsymbol{r}$ & & .0050 & .0003 \\
\hline NEANTIIE. & 12 & BASF & A LLOX & $\mathbf{p}$ & & .0050 & .0001 \\
\hline 13 & 40 & BASt & ALLCY & $\mathbf{P}$ & & .0230 & .0007 \\
\hline MEANTICE & 96 & AASE & ALLOY & $P$ & & .0050 & .4001 \\
\hline 13 & 192 & HASE & ALLOY & $P$ & .079 & .0140 & .0009 \\
\hline MEANTICE & 192 & BASE & ALLOY & P & .041 & .0170 & .0003 \\
\hline TUTAL & 12 & HASE & ALLUY & $\mathbf{P}$ & .0450 & & \\
\hline TOIAL & 36 & BASE & ALLOY & $\mathbf{P}$ & .0060 & & \\
\hline TUTAL & 60 & BASE & ALLOY & $\mathbf{p}$ & .033 & & \\
\hline TOTAL & 120 & BASE & ALLUY & $\mathbf{P}$ & $.0 \$ 9$ & & \\
\hline TOIAL & 12 & BASE & ALLOX & $\mathbf{P}$ & .013 & & \\
\hline TOTAL & 12 & BASE & ALLOY & $\mathbf{P}$ & .015 & & \\
\hline TOTAL & 24 & BASE & ALLOY & $P$ & .045 & & \\
\hline TOIAL & 24 & BASE & ALLUY & $p$ & $.0>0$ & & \\
\hline TOTAL & 60 & BASE & ALLUY & $\mathbf{P}$ & .036 & & \\
\hline TOTAL & 60 & BASE & AL LCY & $\mathbf{P}$ & .020 & & \\
\hline TOTAL & 120 & BASE & ALLCY & $\mathbf{P}$ & .070 & & \\
\hline TOIAL & 120 & UASE & ALLOX & $p$ & .028 & & \\
\hline TOTAL & 12 & GASE & ALLOY & $\mathbf{P}$ & .025 & & \\
\hline
\end{tabular}


TABLE I

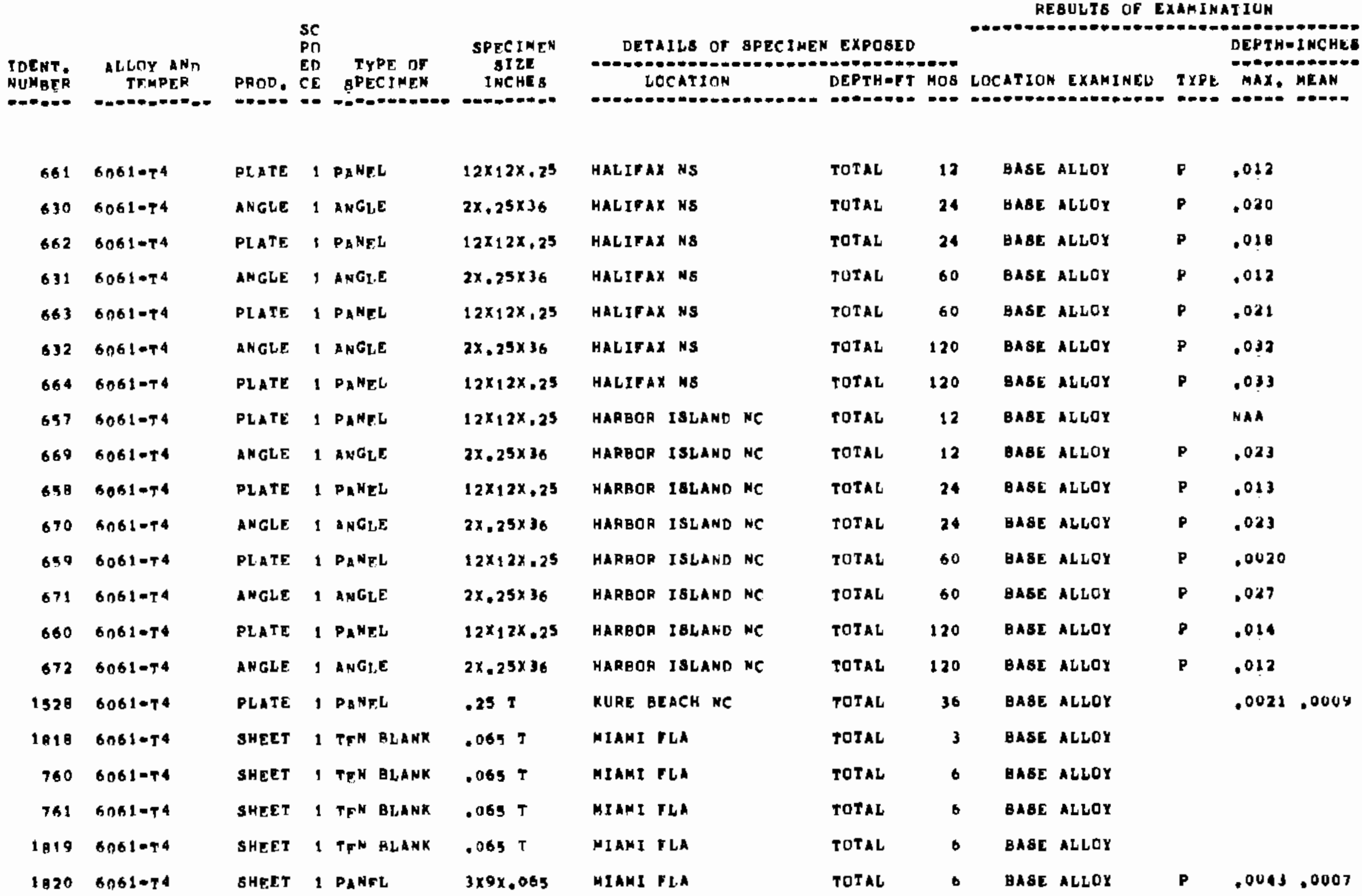


TAPLE I

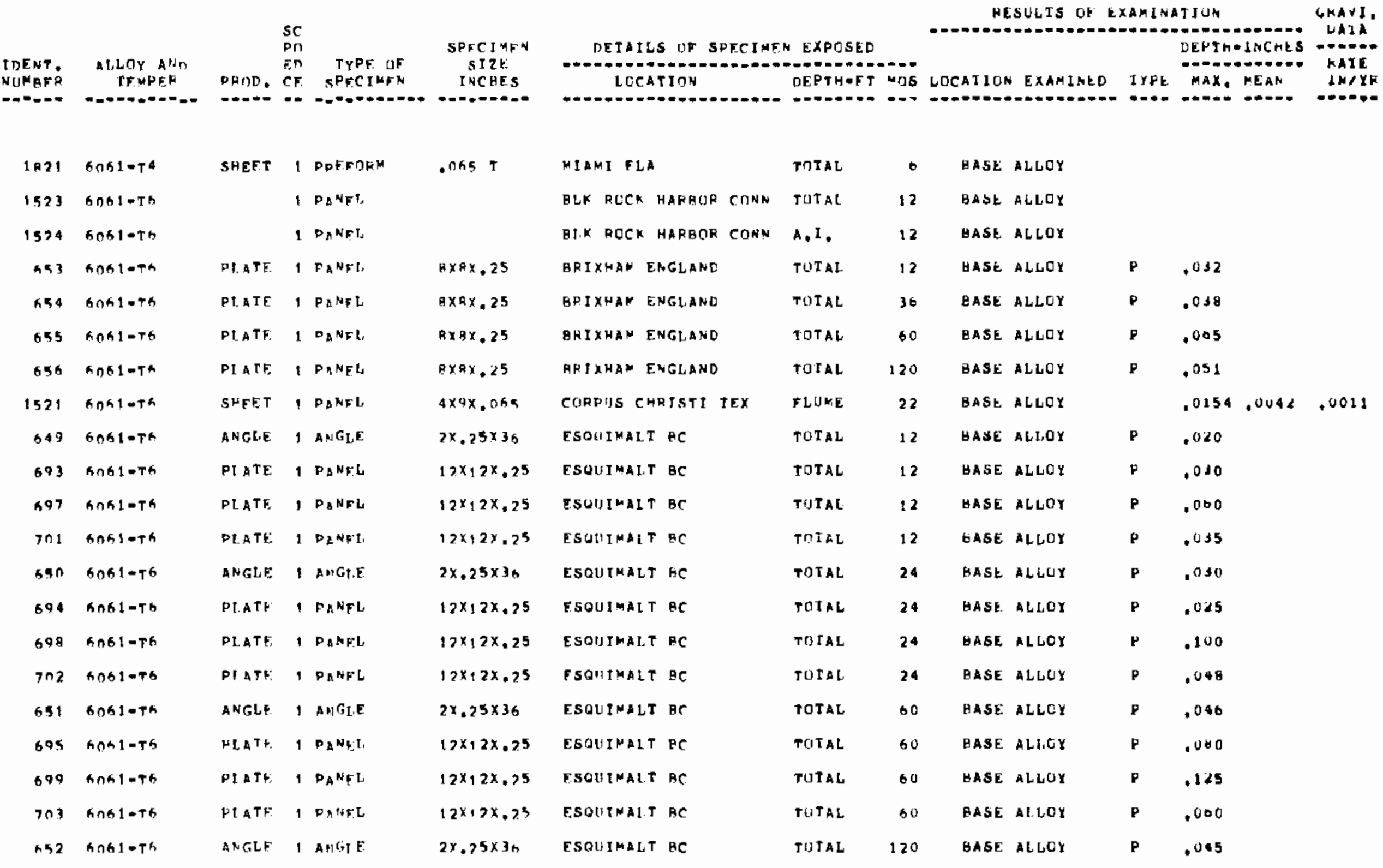


TABLE I

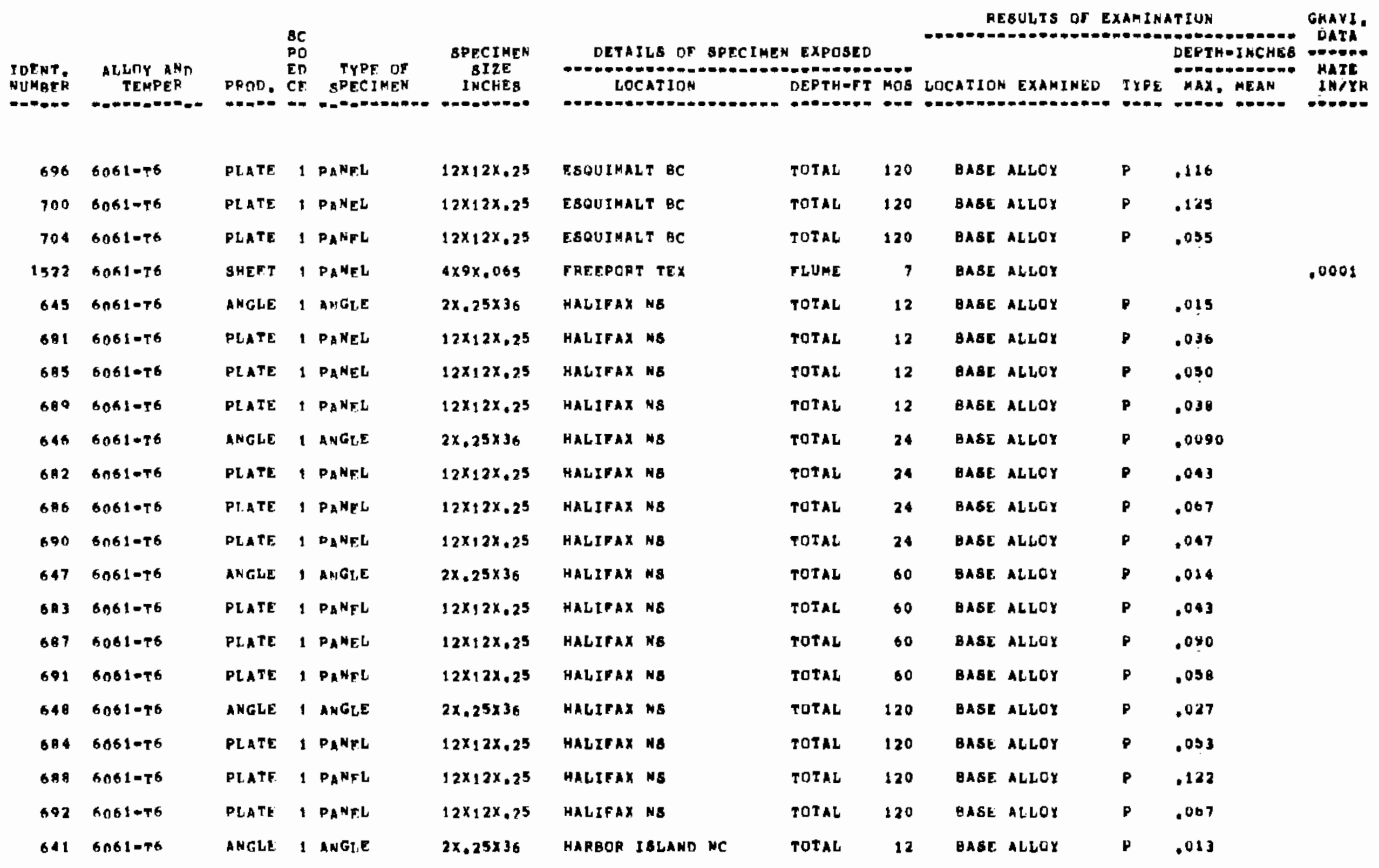


TABLE. I

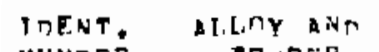
MUNAF TEMPER -...-

\begin{tabular}{|c|c|c|c|c|}
\hline 673 & $\operatorname{son} 1=\tau 6$ & PLATE & 1 & P:NFL \\
\hline 677 & $x \cap 61-T h$ & DL a TE. & 1 & $P_{B} N F L$ \\
\hline 732 & $A \cap A 1-T 6$ & PIATE. & 1 & $P_{D} N F$. \\
\hline 542 & SAS1-Th & $A N C, I E$ & 1 & BAC T, \\
\hline 674 & $6 n t 1=T h$ & PLATF, & 1 & D: \\
\hline 67 月 & $A \cap A D=T h$ & PLATE & 1 & PANE \\
\hline 733 & $\cos 1=T^{6}$ & PI ATE & 1 & $P \Delta N_{F}$ \\
\hline 543 & $\operatorname{AnK1-T^{6}}$ & Anictie & 1 & A. \\
\hline 475 & $A \cap G 1=T G$ & PI ATE & 1 & $D_{\triangle N}$ \\
\hline 679 & 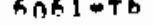 & PLAIE & 1 & DANF \\
\hline 734 & SOK $1=76$ & PLATE & 1 & $P_{1}$ \\
\hline 644 & and $1=7 n$ & $A+G \mathrm{G}, \mathrm{E}$ & 1 & A \\
\hline 676 & GnG $1-76$ & PI ATE & 1 & $P_{A} N$ \\
\hline 500 & $\operatorname{hat} 1=T 6$ & DLATE & 9 & \\
\hline 735 & $6 n D_{1}-\mathrm{T} 6$ & $P\{4 T$ & 1 & $D_{A} N_{1}$ \\
\hline 1472 & AOB $1=T B$ & SHEET & 1 & \\
\hline 1473 & $6061-76$ & SHEET & 1 & \\
\hline 1474 & $6061-76$ & SHEF.T & 1 & \\
\hline 147 & AกA1-TE & $S^{H} E^{F} T$ & $t$ & \\
\hline
\end{tabular}

\begin{tabular}{|c|c|c|}
\hline $\mathrm{Pn}$ & & SDECIMFN \\
\hline $\begin{array}{l}E^{n} \\
C F\end{array}$ & $\begin{array}{r}\text { TYPF UF } \\
\text { SDECI I EEN }\end{array}$ & $\begin{array}{l}\text { SIZE } \\
\text { IVCHES }\end{array}$ \\
\hline
\end{tabular}
DFTAILS IF SPECTMEN EXPTSED DEPTH=INCHES DATA

HESULTS UF EXAMIMATIUM GaAVI. LOCATION OEPTHAT MOS

DEPTH-FT MOS LOCAIIOM EXAMINEL IYPL MAX MEAH KATE

\begin{tabular}{|c|c|c|}
\hline $12 \times 12 \times, 25$ & RAREOP & ISLAND \\
\hline $12 \times 12 \times .25$ & HAPHEP & ISLANR \\
\hline $1>x_{1} 2 \times, 25$ & HARBCP & ISLAND \\
\hline $2 \times .25 \times 36$ & HARHOK & I SLA $B$ \\
\hline $12 \times 12 \times .25$ & HARRDP & ISLAND \\
\hline $12 \times 22 x \cdot 25$ & FARHOP & ISLAND \\
\hline $12 \times 17 \times, 75$ & HARHCW & ISt,AND \\
\hline $2 \times .25 \times 36$ & HARAOL & ISLAND \\
\hline $12 \times 12 \times .25$ & HAREOP & ISLANO \\
\hline $12 \times 12 \times .25$ & HAKBOA & ISLAND \\
\hline $12 x_{1} 2 x_{.25}$ & HARRUK & ISLAND \\
\hline $2 \times .25 \times 36$ & HARBCF & ISLANO \\
\hline $12 x ! 2 x, 25$ & HARBOP & ISLAND \\
\hline $12 \times, 2 x, 25$ & HAFBDE & ISLAND \\
\hline $12 \times 12 \times, 25$ & HARBOP & ISLAND \\
\hline $.125 \mathrm{I}$ & HUF,NEMT. & CAL \\
\hline $.125 \mathrm{~T}$ & HUENEME & CAL \\
\hline $.125 \mathrm{~T}$ & HUEAFME & $\mathrm{CAI}$ \\
\hline 5 & $\mathbf{E}$ & $C$ \\
\hline
\end{tabular}

\begin{tabular}{|c|c|c|c|c|c|c|c|}
\hline TOTAL & 12 & GA5E & ALLU & $\mathbf{P}$ & .007 & & \\
\hline TUIAL & 12 & HASE & ALLOY & $\mathbf{P}$ & .015 & & \\
\hline TOTAL & 12 & GASE & ALLOY & $\mathbf{P}$ & .036 & & \\
\hline TUTAL & 24 & DASE & ALLCY & $\mathbf{P}$ & .013 & & \\
\hline TOTAL & 24 & BASE & ALLOX & $\mathbf{p}$ & .200 & & \\
\hline TUTAL & 24 & BASE' & ALLOY & $\mathbf{P}$ & .027 & & \\
\hline TUTAL & 24 & BASE & $A L L O Y$ & $\mathbf{P}$ & .044 & & \\
\hline TOTAL & 60 & EASE & ALLOY & $\mathbf{P}$ & .020 & & \\
\hline TUTAL & bo & GASE & ALLCY & $p$ & .144 & & \\
\hline TUTA L & 60 & BASE & ALLOX & $\mathbf{P}$ & .036 & & \\
\hline TOTAL & 60 & BASE. & ALLOY & $\mathbf{p}$ & .000 & & \\
\hline TOT A L & 120 & HASE & ALLUY & $\mathbf{P}$ & .015 & & \\
\hline TOTAL & 120 & HAGE & ALLUX & $\mathbf{p}$ & .130 & & \\
\hline TOTAL & 120 & BAEE & ALLOY & $\mathbf{P}$ & .040 & & \\
\hline TOTAL & 120 & GASE & ALLOY & $\mathbf{P}$ & .045 & & \\
\hline 5640 & 4 & BASE & $A L L O X$ & $\mathbf{P}$ & I NC I P & & .0041 \\
\hline 5080 & 4 & BASE & ALLOY & $P$ & .0050 & .0018 & \\
\hline $50408 k 0$ & 4 & BASE & ALLUYY & $\boldsymbol{p}$ & .032 & .022 & .0009 \\
\hline 5640 SED & 4 & OASE. & ALLUX & $\mathbf{P}$ & .031 & .0256 & .0016 \\
\hline
\end{tabular}


TABLE I

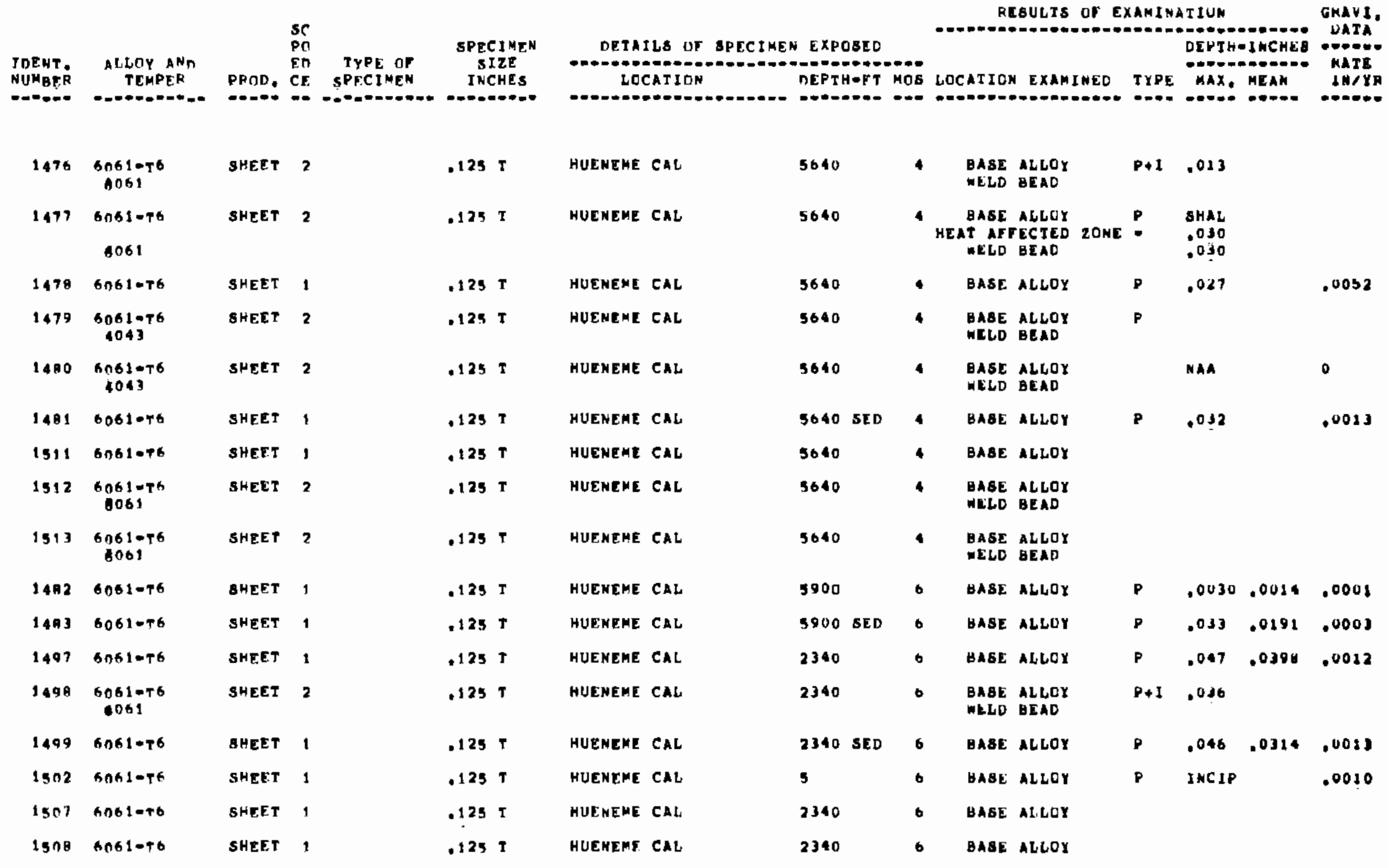


TAELE 1

\begin{tabular}{|c|c|c|c|c|c|c|c|c|c|c|c|c|c|c|c|c|}
\hline $\begin{array}{l}\text { PTFNT } \\
\text { NISMER }\end{array}$ & $\begin{array}{c}\text { ALLTY ANp } \\
\text { TFMDER }\end{array}$ & PRחIS, & $\begin{array}{l}\text { sr } \\
\text { Pn } \\
\text { En } \\
\text { CF }\end{array}$ & $\begin{array}{r}\text { TYPF PF } \\
\text { SPFCIMEV }\end{array}$ & $\begin{aligned} 5 P 5 C \\
\text { INT } \\
\text { INC }\end{aligned}$ & $\begin{array}{l}\text { CIMFN } \\
\text { TZE } \\
\text { CHES }\end{array}$ & $\begin{array}{r}\text { DETAI } \\
\text { LCO }\end{array}$ & $\begin{array}{l}\text { ILS DF BFECIMEN } \\
\text { CATION }\end{array}$ & $\begin{array}{l}\text { EXPOSEL } \\
\text { DEPTH-FT }\end{array}$ & mis & 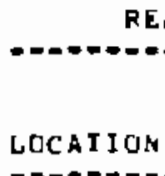 & ESULIS OR E & XAMIND & $\begin{array}{l}\text { IIUN } \\
\text { DEPTH } \\
\text { MAX. }\end{array}$ & $\begin{array}{l}\text { LNCHES } \\
\text { MEAN }\end{array}$ & $\begin{array}{l}\text { GMAVI. } \\
\text { WATA } \\
\text { KATL } \\
\text { DH/XK }\end{array}$ \\
\hline 1517 & $\operatorname{AnA} 1-T^{6}$ & SHEFT & 1 & & .125 & $\mathrm{~T}$ & HEJENERE & CAL & 2340 & $b$ & BASk & ALLOY & & & & \\
\hline $159 \mathrm{~A}$ & $\operatorname{Ron} 1=T h$ & SHEFT & 1 & & .125 & $T$ & HHENEME & CAL & 2340 & 6 & $\forall A S E$ & ALLOY & & & & \\
\hline 1570 & $A \cap B 1=T h$ & SHEFT & $t$ & & .125 & $\mathrm{I}$ & HIJENENE & CAl, & 5 & 6 & BASE & ALLOY & & & & \\
\hline 1424 & $A n A 1=T h$ & SHEET & 1 & & .125 & $\mathrm{I}$ & HUENEPF & CAI, & 6780 & 13 & BASE & ALLOY & $\mathbf{P}$ & .058 & .0464 & .0010 \\
\hline $14 R 5$ & anstoth & SHEET & 1 & & .125 & $\top$ & HUENEFE & CAL & 6780 SED & 13 & BASE & ALLDY & P & .000 & .0453 & .0007 \\
\hline 1500 & $6051=T^{h}$ & SHEF.T & 1 & & .125 & ${ }^{\top}$ & HUENEME & CAL & 2370 & 13 & $\forall A S_{E}$ & ALLOY & $\mathbf{p}$ & .075 & .0515 & .0020 \\
\hline $150 \%$ & GnK $1=T 6$ & SHEFT & 1 & & .125 & $\mathrm{~T}$ & HUFNENE & CAL & 2310 SED & 13 & UASE & ALLOY & $\mathbf{P}$ & .076 & .0513 & .0012 \\
\hline $15 \cap 3$ & GDS1-Th & SHEFT & 1 & & .124 & $\tau$ & HL'ENEME & CAI & $\mathbf{5}$ & 13 & $\begin{array}{l}\text { BASE } \\
\text { UASE }\end{array}$ & $\begin{array}{r}\text { ALLQY } \\
\text { E EDGE }\end{array}$ & $\begin{array}{l}P \\
=\end{array}$ & $\begin{array}{l}.016 \\
\text { YES }\end{array}$ & .014 & .0007 \\
\hline 1505 & $\operatorname{Ang} 1=T 6$ & SHEET & 1 & & .125 & $T$ & HUENEME & CAL & 6780 & 13 & BASE & ALLUY & & & & \\
\hline $15 n 6$ & $A 061=T^{6}$ & SHEFT & + & & .175 & $\mathbf{T}$ & HUENENE & CAL & 6780 & 13 & BASE & ALLOY & & & & \\
\hline 1500 & $h \cap G 1=+b$ & SHEF? & 1 & & .125 & $\tau$ & HUENEME & CAL & 2310 & 13 & BASE & ALLDY & & & & \\
\hline 1510 & $6 n k 1=T^{6}$ & $S^{H E F} \mathbf{T}$ & 1 & & .125 & $\mathbf{T}$ & HUENEYE. & CAL & 2370 & 13 & UASE & ALLUY & & & & \\
\hline 1519 & $h \cap A T=T^{n}$ & SHERT & 1 & & .125 & $\mathrm{~T}$ & HUENEME & $C A L$ & 6780 & 13 & BASE & ALLOY & & & & \\
\hline 1519 & $A n B 1=T 6$ & SHEF:T & 1 & & .125 & $\mathbf{T}$ & HUEENENE & CAL & 2370 & 13 & BASE & ALLUY & & & & \\
\hline 1504 & $G \cap A 1=T 6$ & $S H F F T$ & 1 & & .125 & $T$ & HUENEFE & CAL & $\mathbf{5}$ & 10 & BASE & AL LOY & $\mathbf{P}$ & .023 & .0101 & .0003 \\
\hline $14 R h$ & hok $1-T h$ & $S H F, F$ & 1 & & .125 & ${ }^{\top}$ & HUFNEME. & CAL & 5040 & 24 & $\begin{array}{l}\text { GASE } \\
\text { GASE }\end{array}$ & $\begin{array}{l}\text { ALLOY } \\
E \text { EDGE }\end{array}$ & $\begin{array}{l}F \\
-\end{array}$ & $\begin{array}{l}\text { OOOS } \\
X E B\end{array}$ & .0420 & .0004 \\
\hline 1497 & $\begin{array}{l}\text { hanileth } \\
\text { anb } 1\end{array}$ & SHE F. T & 2 & & +125 & $\mathrm{~T}$ & HIE.NEMF & CAL & 5640 & 24 & $\begin{array}{l}\text { BASE } \\
\text { WELD }\end{array}$ & $\begin{array}{l}\text { ALLOY } \\
\text { BEAL }\end{array}$ & & & & \\
\hline 149 & $n n k 1-T^{n}$ & SLF't. T & 1 & & .125 & $\mathrm{I}$ & मUFAEME & CAI & $5 \times 40$ & 24 & $\begin{array}{l}\text { BASF } \\
\text { YASE }\end{array}$ & $\begin{array}{l}\text { ALLCY } \\
E \quad \text { EBGE }\end{array}$ & 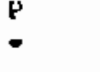 & $\begin{array}{l}\text { OUt } 3 \\
\text { SEVER }\end{array}$ & & $.00<6$ \\
\hline 1449 & $A \cap A 1 * T^{h}$ & SHET & 1 & & .124 & ${ }^{\top}$ & HUFAFMF & CAL & 5640 & 24 & HASE' & ALLEY & $\mathbf{p}$ & , uso & .0434 & .0018 \\
\hline
\end{tabular}


TABLE I

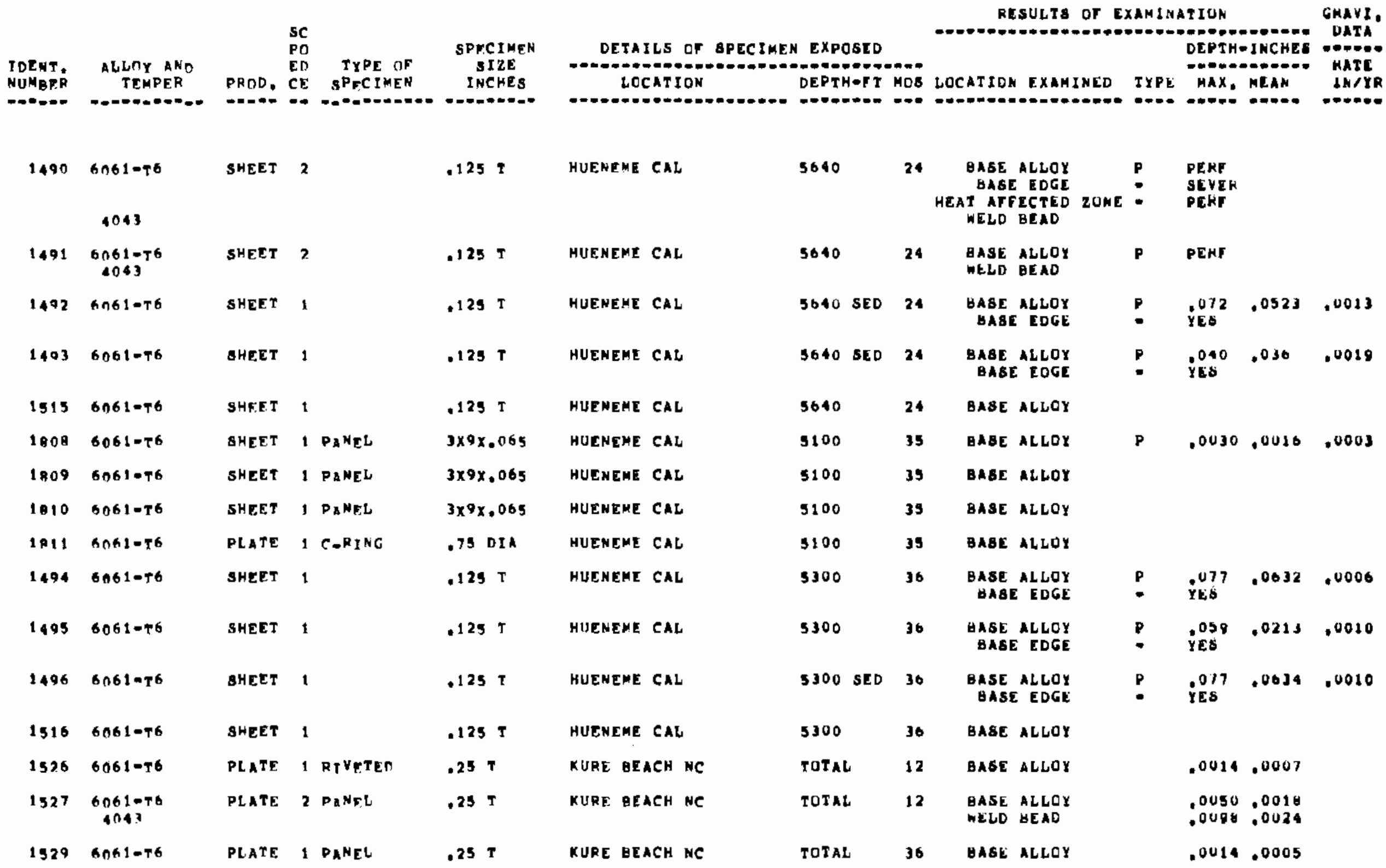


TAELE I

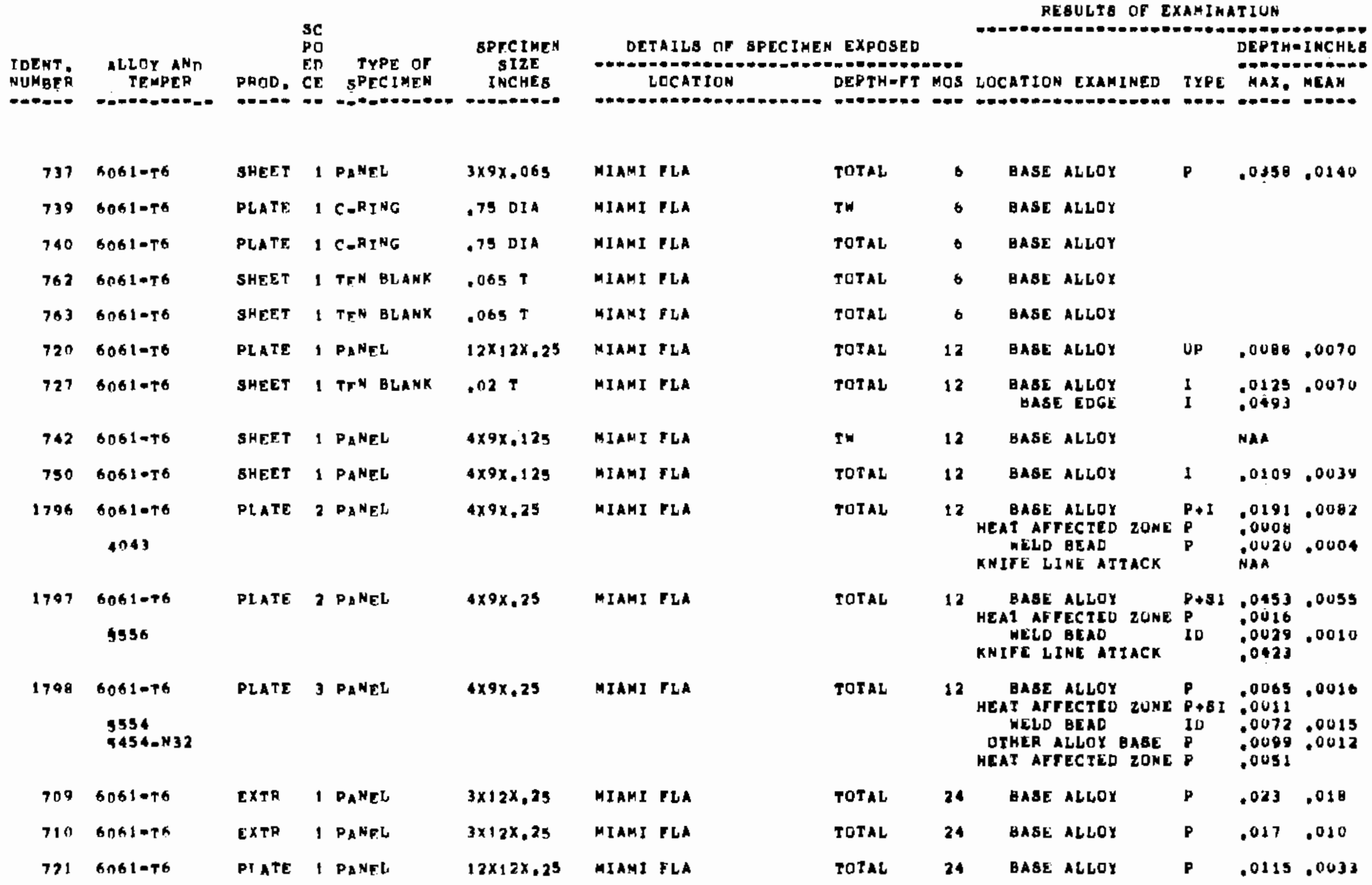


TABLE I

\begin{tabular}{|c|c|c|c|c|c|c|c|c|c|c|c|c|c|c|}
\hline $\begin{array}{l}\text { IDENT: } \\
\text { RUMBER }\end{array}$ & $\begin{array}{l}\text { LLOY AND } \\
\text { TENPEP }\end{array}$ & PAOD, & $\begin{array}{l}\text { SC } \\
\text { PO } \\
\text { ED } \\
\text { CE }\end{array}$ & $\begin{array}{l}\text { TYPF OF } \\
\text { SPFCIMEN }\end{array}$ & $\begin{array}{l}\text { SPECIMEH } \\
\text { SIZE } \\
\text { TNCHES }\end{array}$ & DEt & $\begin{array}{l}\text { TAILS OF BPECINEN } \\
\text { LOCATIUN }\end{array}$ & $\begin{array}{l}\text { EXPOSED } \\
\text { DEPTH=TT }\end{array}$ & MOS & RESULTS OF EX & $x A M 1 N A$ & $\begin{array}{l}\text { TIUN } \\
\text { DEPTHE } \\
\text { MAX. }\end{array}$ & $\begin{array}{l}\text { INCHES } \\
\text { MEAN } \\
\text { MEA- }\end{array}$ & $\begin{array}{l}\text { GRAYI, } \\
\text { DATA } \\
\text { POATE } \\
\text { HATE } \\
\text { IN/YH }\end{array}$ \\
\hline 1903 & $\begin{array}{l}\text { SOA1 }=76 \\
5556\end{array}$ & PLATE & 2 & $D_{A} \mathrm{NE}_{\mathrm{C}}$ & $4 \times 9 \times, 25$ & MIAMI & FLA & TatAL & 36 & $\begin{array}{l}\text { BAGE ALLOY } \\
\text { HEAT AFEECTED ZONE } \\
\text { WELD GEAO } \\
\text { KMIFE LINE TTACK }\end{array}$ & $\begin{array}{l}\text { I } \\
\text { ID }\end{array}$ & $\begin{array}{l}.0460 \\
.0054 \\
.0068 \\
.0300\end{array}$ & $\begin{array}{l}.0064 \\
.0041 \\
.0054\end{array}$ & \\
\hline $1 \mathrm{Ant}$ & $\begin{array}{l}5061-76 \\
5554 \\
9454-432\end{array}$ & PI.ATE & 3 & PANFL & $4 \times 9 \times .5$ & MIAMI & FLA & TOTAL & 36 & $\begin{array}{l}\text { BASE ALLOY } \\
\text { HEAT ATFECTED ZONE } \\
\text { WELD BEAD } \\
\text { KNIFE LINE ATIACK } \\
\text { OTHER ALLOY BASE } \\
\text { HEAT AFFECTED ZONE }\end{array}$ & $\begin{array}{l}p \\
p+1 \\
p \\
p \\
p\end{array}$ & $\begin{array}{l}.0046 \\
.0020 \\
.0046 \\
.0027 \\
.0068 \\
.0014\end{array}$ & $\begin{array}{l}.0019 \\
.0010 \\
.0019 \\
.0027 \\
.0005\end{array}$ & \\
\hline 713 & $6061-78$ & EXTA & 1 & PANEL & $3 \times 12 \times, 25$ & IANI & FLA & TOTAL & 48 & BASE ALLOY & $p$ & .046 & .039 & \\
\hline 714 & $6 \cap 61-76$ & EXTR & 1 & PaNFL & $3 \times 12 \times .25$ & MIAHI & TLA & TOTAL & 48 & BAGE ALLOY & $\mathbf{p}$ & .033 & .020 & \\
\hline 723 & $\operatorname{son} 1=T^{6}$ & PLATE & 1 & PANEL & $12 \times 12 \times .25$ & MIAMI & FLA & TOTAL & 48 & BABE ALLOY & UP & .0224 & .0007 & \\
\hline 745 & $6 n s 1=76$ & BHEET & 1 & PANEL & $4 \times 9 \times, \pm 25$ & MIAMI & FLA & $\mathbf{T W}$ & 48 & BASE ALLOY & I & .0042 & .0021 & \\
\hline 753 & $\ln 81-76$ & EHEFT & 1 & DANTL & $4 \times 9 \times, 125$ & MIAM I & FLA & TOTAL & 10 & BASE ALLOY & I & .0290 & .0031 & \\
\hline 758 & SOA1-56 & ANGLE. & 1 & $A N G_{1}, E$ & $2 \times 2 \times 12$ & MIAMI & FLA & Tin & 48 & BA6E ALLOY & $\mathbf{P}$ & .0022 & .0027 & \\
\hline 799 & $\operatorname{sn} x 1=T 6$ & ANGLE & 1 & $A N G T, E$ & $2 \times 2 \times 12$ & MIAMT & F1A & TOTAL & 48 & BASE ALLOY & $\mathbf{P}$ & .0265 & .0130 & \\
\hline 1 คก 5 & $\begin{array}{l}6 n 61-76 \\
4043\end{array}$ & PI ATF & 2 & PANFL & $4 \times 9 \times, 25$ & MIAMT & FLA & TDTAL & 48 & $\begin{array}{l}\text { BABE ALLOY } \\
\text { HEAT AFTECTED ZONE } \\
\text { WELD BEAD }\end{array}$ & $\begin{array}{l}l \\
\text { ID }\end{array}$ & $\begin{array}{l}.0140 \\
.0028 \\
.0018\end{array}$ & $\begin{array}{l}.0069 \\
.0006 \\
.0013\end{array}$ & \\
\hline $1 B \cap 6$ & $\begin{array}{l}3061-T^{6} \\
3556\end{array}$ & PLATE & 2 & PANFL & $4 \times 9 \times, 25$ & MIAMI & FLA & TOTAL & 48 & $\begin{array}{l}\text { BASE ALLOY } \\
\text { HEAT AFFECTED ZONE } \\
\text { WELD BEAD }\end{array}$ & $\begin{array}{l}I \\
P+5 I \\
10\end{array}$ & $\begin{array}{l}.0228 \\
.0092 \\
.0158\end{array}$ & $\begin{array}{l}.0070 \\
.0035 \\
.0116\end{array}$ & \\
\hline 1807 & $\begin{array}{l}6061=T^{6} \\
9554 \\
2454-H 32\end{array}$ & PLATE & 3 & $D_{A} N_{E} I_{L}$ & $4 \times 9 \times .5$ & MIAMI & FLA & TOTAL & 48 & $\begin{array}{l}\text { BASE ALLOY } \\
\text { HEAT AFFECTED ZUNE } \\
\text { HELD READ } \\
\text { OTHEA ALLOY BASE }\end{array}$ & $\begin{array}{l}\text { UP } \\
\mathbf{P}\end{array}$ & $\begin{array}{l}.0035 \\
.0005 \\
\text { NAA }\end{array}$ & $\begin{array}{l}.0021 \\
.0004\end{array}$ & \\
\hline $7+5$ & $6061-76$ & $E \times T R$ & 1 & $P_{A} N F$ & $3 \times 12 \times .23$ & MIAMI & FLA & TOTAL & 60 & DASE ALLOY & $\mathbf{P}$ & .002 & .051 & \\
\hline
\end{tabular}


TARIE I

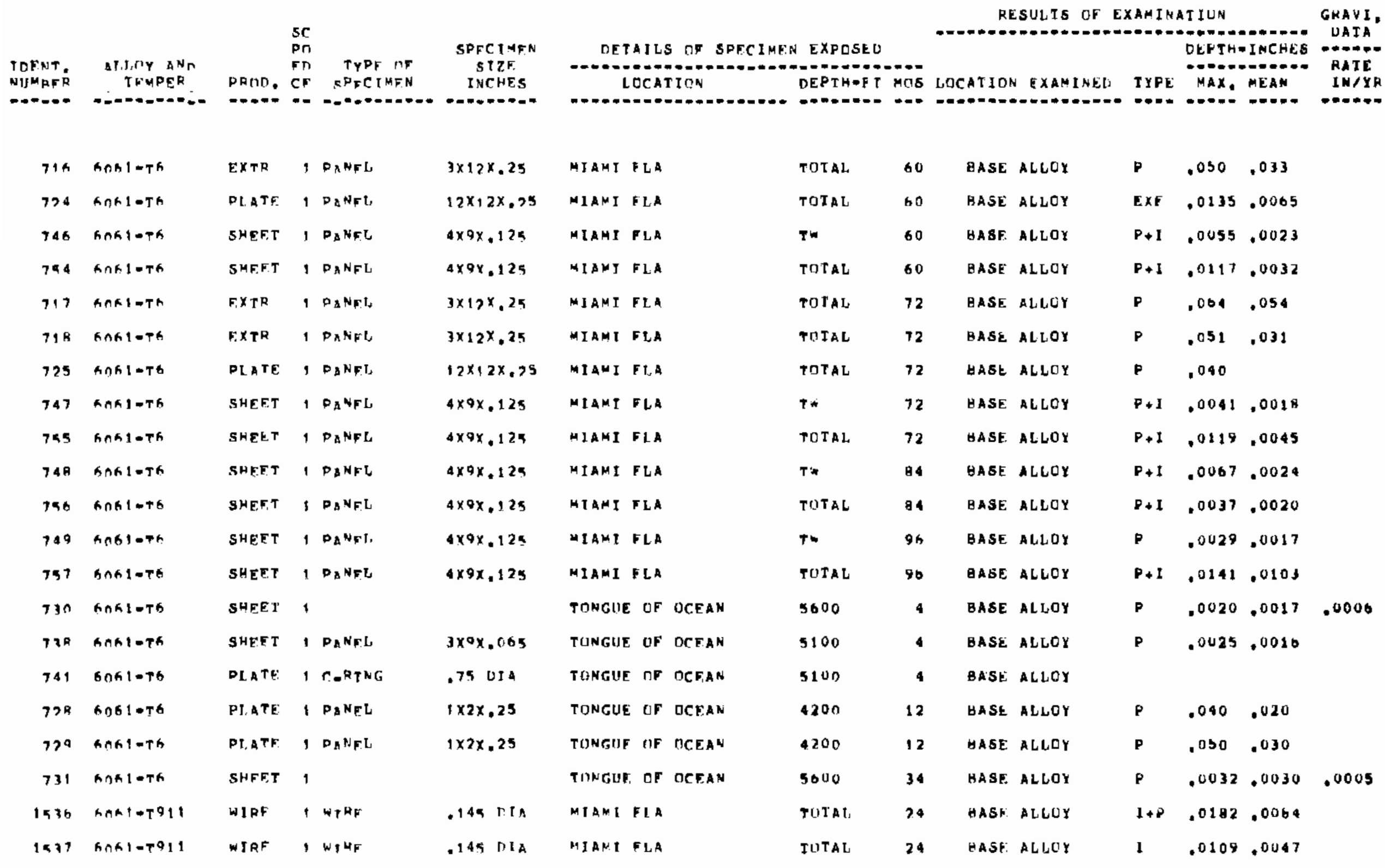


TAALE I

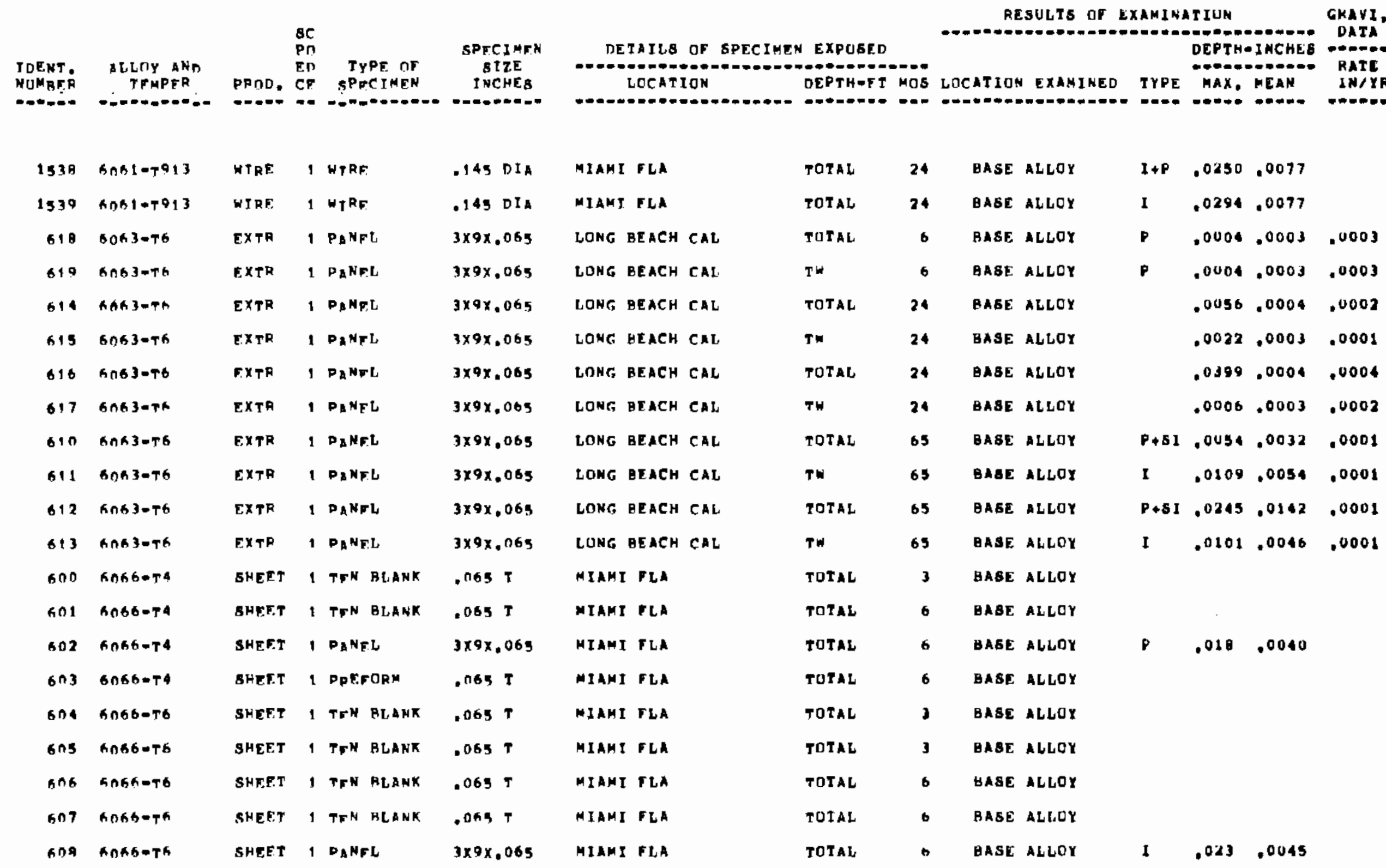


TABLE I

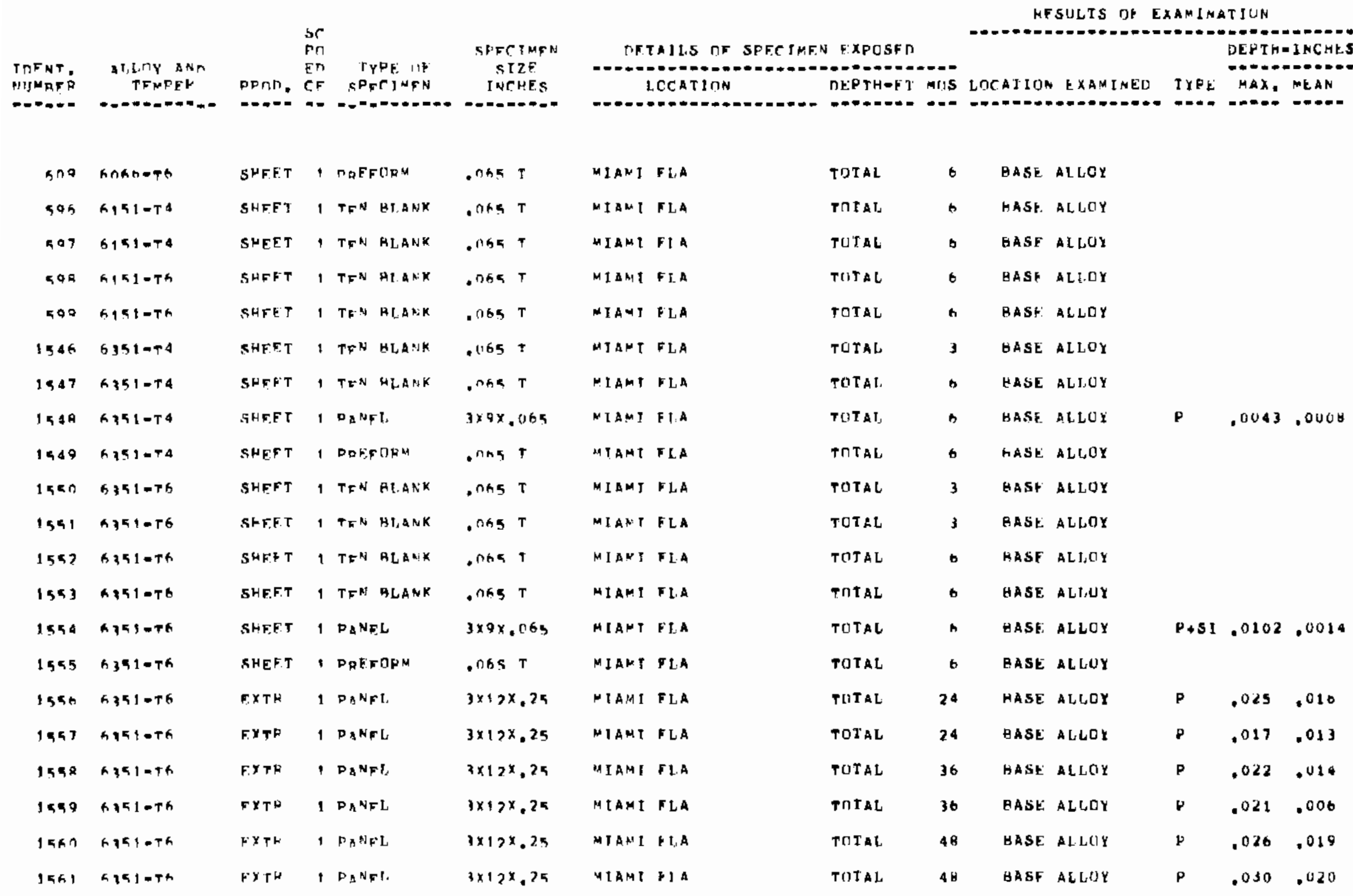


TABLE I

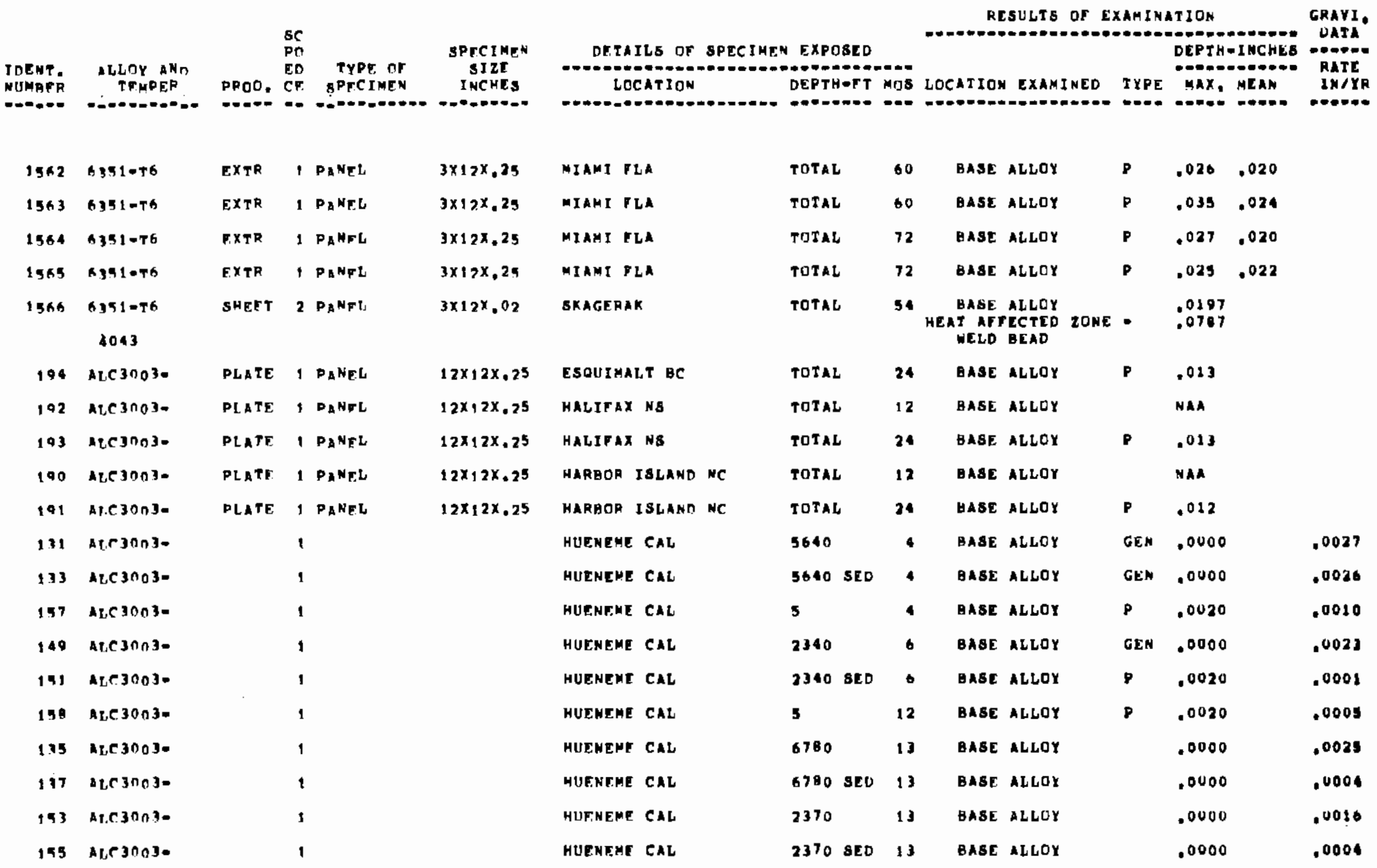


TABLF I

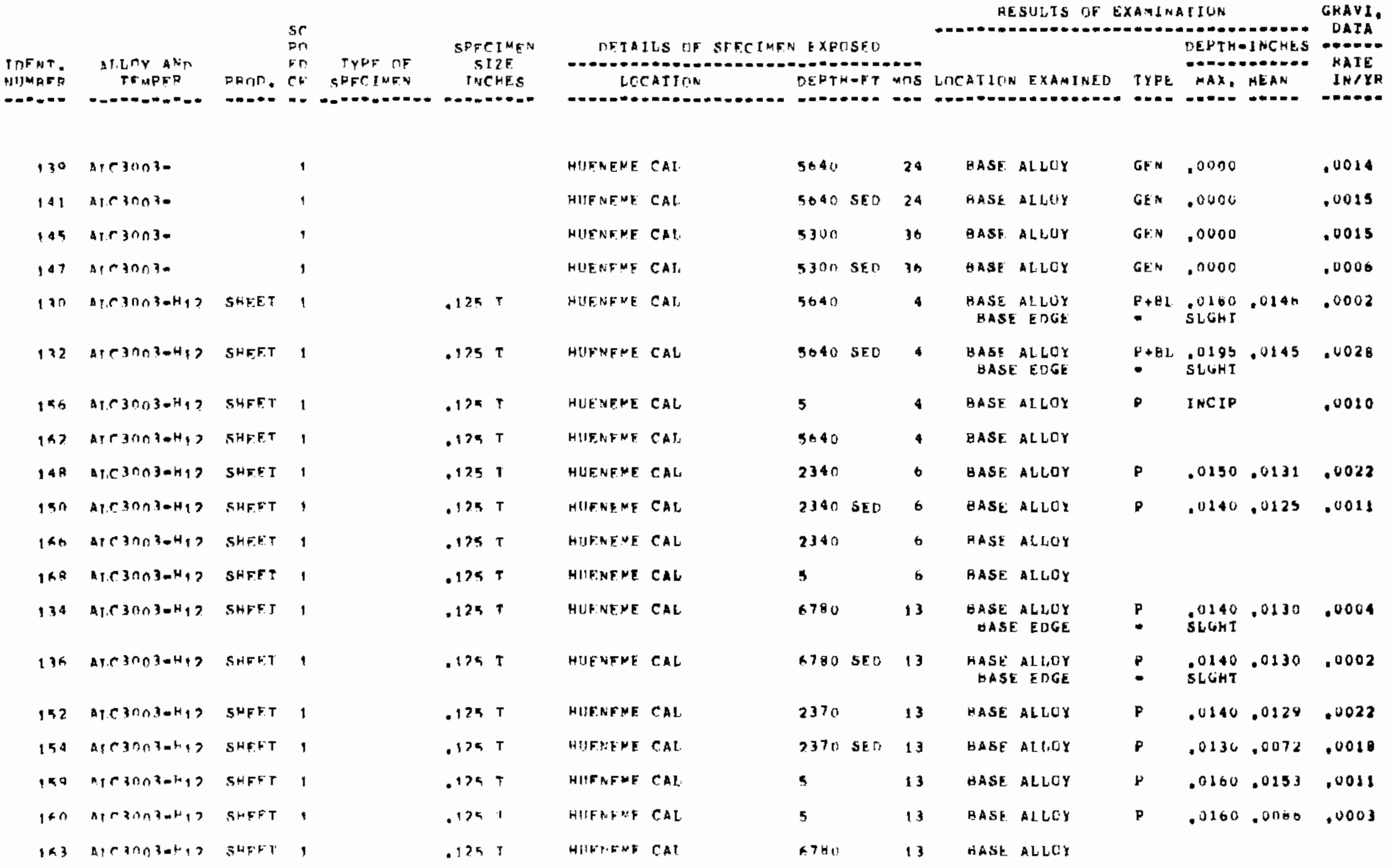


TABLE I

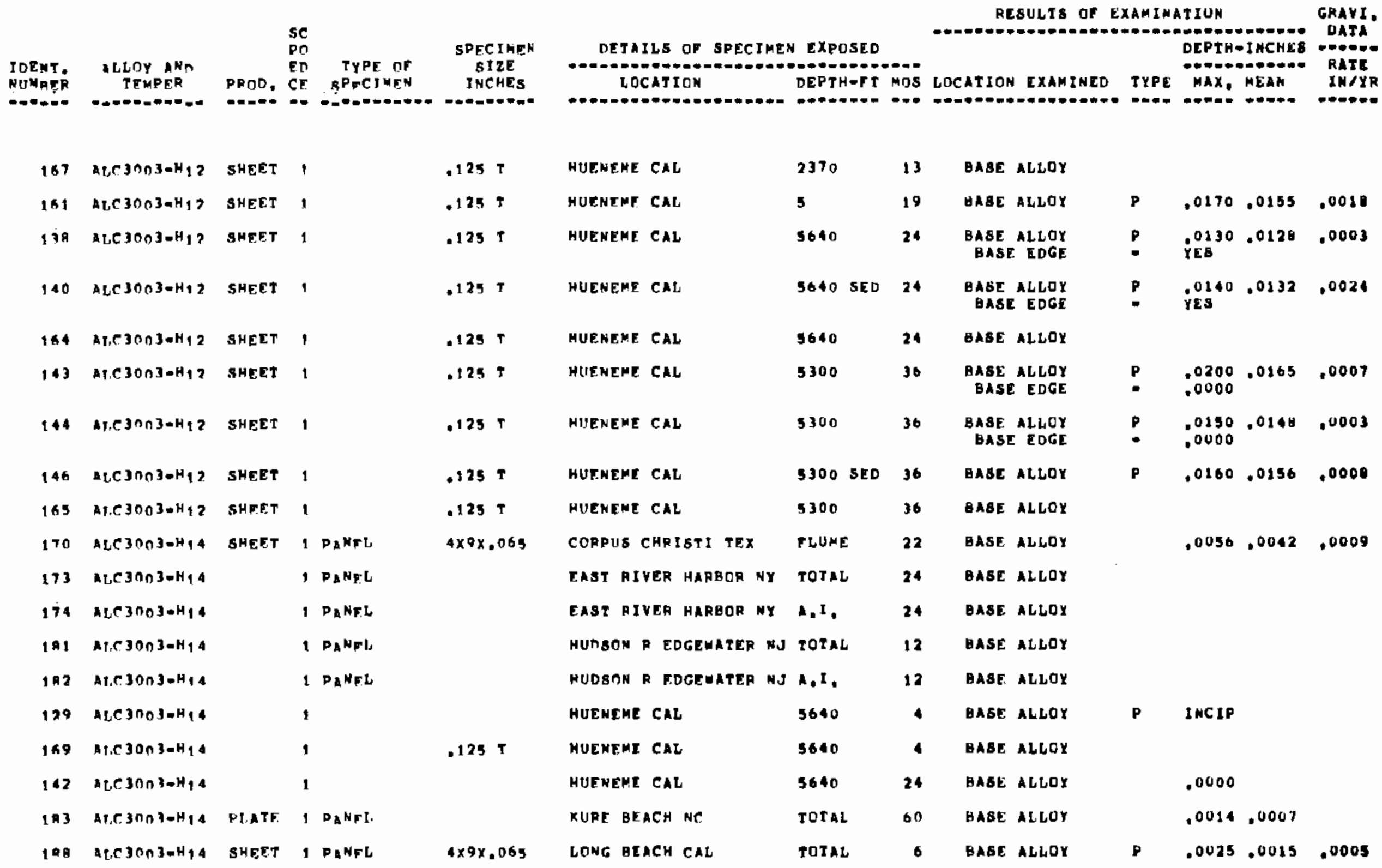


TABLE I

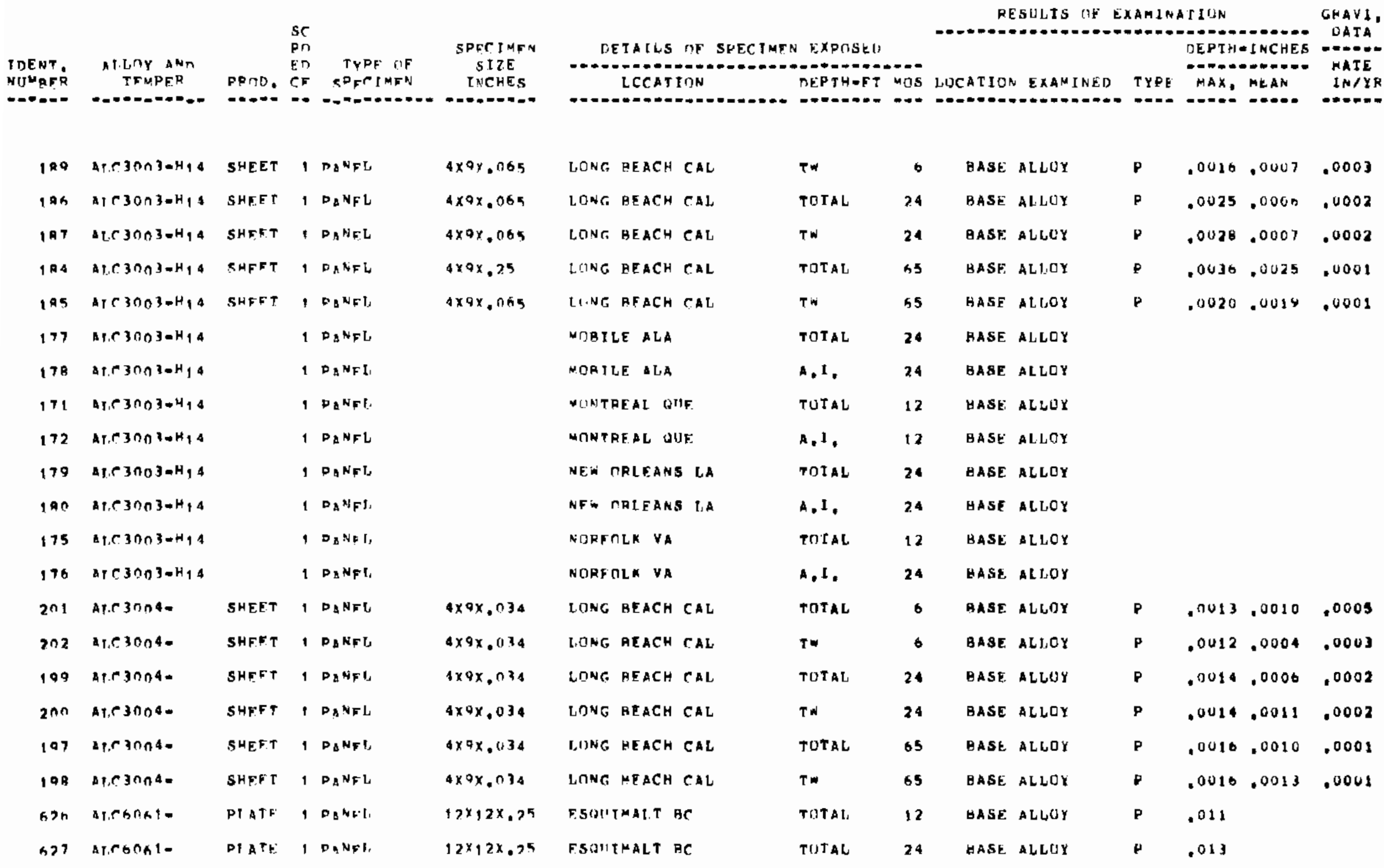


TAALE I

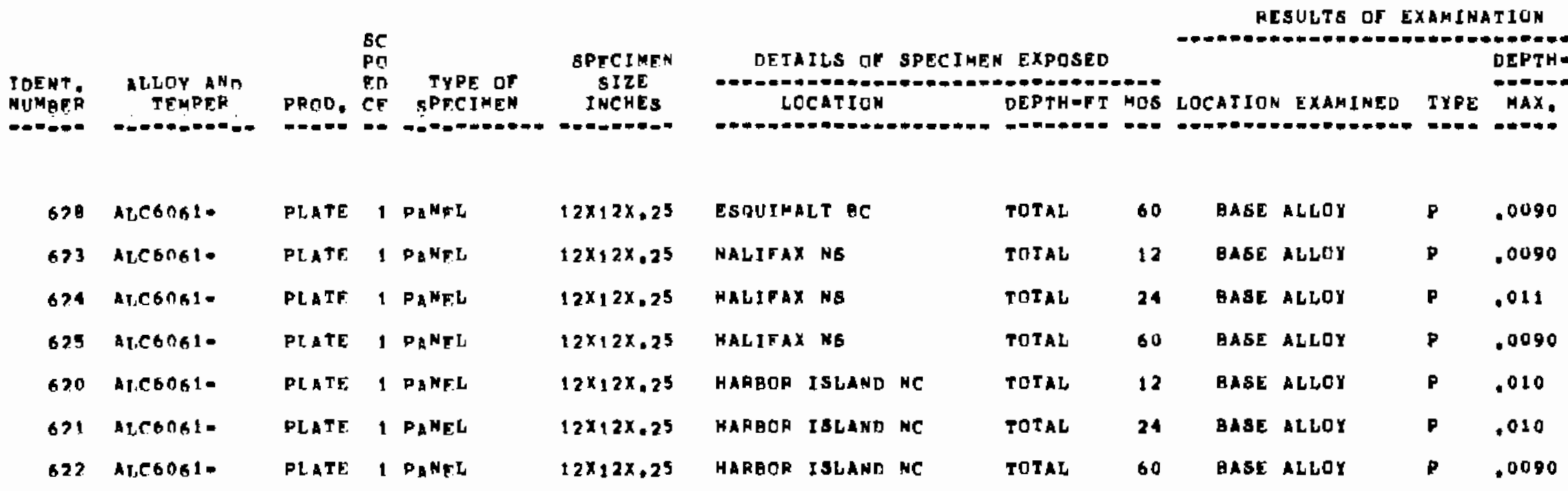




\section{Elumn Heading}

[dent. Number uetal Condition

\section{AFGR}

Crevice Attack IREQ PIT/SQCM

3tress

Dorrosion

Change in Property Me thod Used

to Measure PITS

Sea water

Exposure

Condition

Reference

\section{Explanation of Abbreviations}

IDENT = Identification

Vapor DEG = Vapor Degreased; ACET CLEAN = Acetone Cleaned; CHEM Clean = Chemically Cleaned; 1 WK $100 \mathrm{C}=$ Heated 1 week at $100^{\circ} \mathrm{Centegrade}$

MFGR = Manufacturer; ALCOA = Aluminum Company of America; ALCAN = Aluminum Company of Canada; REY = Reynolds Metals Company

MAX = Maximum; PERF = Perforated; INCIP = Incipient; SEVRE = Severe; PF = Perforated $\mathrm{FREQ}=$ Frequency; $\mathrm{SQCM}=$ Square Centimeter

KSI $=$ Kips per Square Inch $(1 \mathrm{Kip}=1000$ pounds $) ; \mathrm{F} / \mathrm{N}=$ Ratio of Specimens Failed to Specimens Exposed

T.S. = Tensile Strength; EL = Elongation

PIT GAGE = Pit Depth Gage; X-SECT. = Cross-Section Examined by Metallographic Technique; MICROSC. = Microscope.

FT/SEC = Feet per Second; TEMP (C) = Temperature in Degrees Centigrade $\mathrm{ML} / \mathrm{L}=$ Milliliters per Liter; $\mathrm{PPM}=$ Parts Per Million; Barnac = Barnacles $\mathrm{ALCOA}=\mathrm{Aluminum}$ Company of America; LIT = Literature 
, 
TAHLF II

MJSCRLLANEUHS CTRRCSTUN INHCPASTION cPEvick CPEVICF CHANGE IA

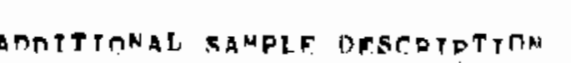
TTACK STRESS PROPEATY METHODO

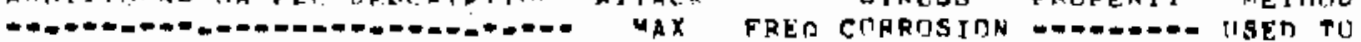

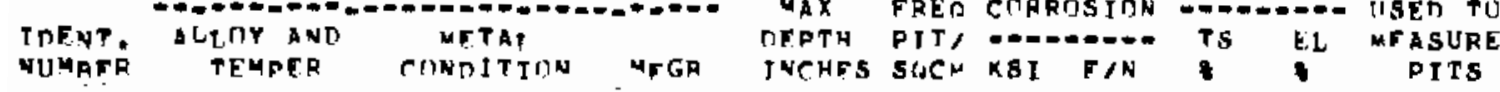

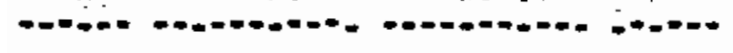

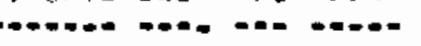

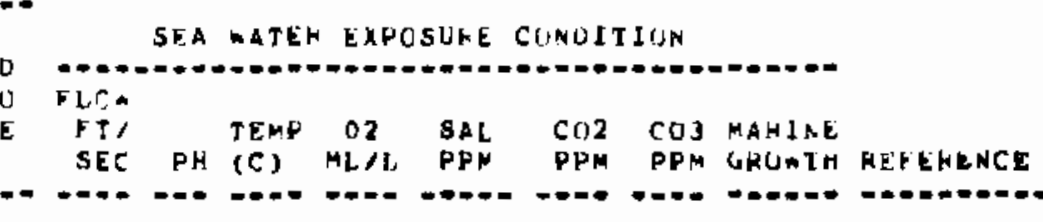

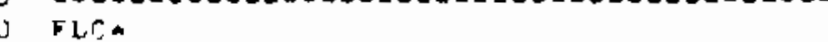

\begin{tabular}{|c|c|c|c|}
\hline 38 & $\ln (n)-4,4$ & MACHINER & $\therefore \mathrm{LCOA}$ \\
\hline 74 & $\ln A_{1} O=H_{1} 4$ & YACHTNET & $\triangle$ LCOA \\
\hline 35 & $1060=H_{1} 4$ & MACHINFE & AlCCA \\
\hline 36 & $1060-H_{1} 4$ & MACHINEN & ${ }_{A} \mathrm{l}_{C} \mathrm{CHA}$ \\
\hline 39 & $11 \cap n-$ & VAPTR DET; & \\
\hline 40 & $110 n=$ & VAPRA DEC. & \\
\hline 41 & $11 \mathrm{nn}=$ & VAPTIR DEC; & \\
\hline 12 & $1100=$ & VAPTR GFG & \\
\hline 43 & $1100=$ & VAPCA DFG & \\
\hline 44 & $\ln \ln =$ & VAPTD ner; & \\
\hline 47 & & & \\
\hline 49 & 11000 & & \\
\hline 45 & 11 no- $F$ & MACHINER & 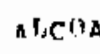 \\
\hline 46 & $11 \cap n=F$ & MILL FINISH & at: t. \\
\hline 61 & $110 n-H_{14}$ & & \\
\hline 62 & $\ln n n=414$ & & \\
\hline h 3 & $\mathrm{IfAO}=\mathrm{H}, \mathrm{A}$ & & \\
\hline 64 & $\ln _{n \rightarrow-N 14}$ & & \\
\hline 5 & $1 \ln (1)+y_{14}$ & & Alican \\
\hline $5 \times$ & 1100101144 & & $\operatorname{ATCAN}$ \\
\hline 59 & $11 n n-H_{1} 4$ & & A lCAN \\
\hline an & $110 n=H_{11}$ & & $A T, \Gamma A N$ \\
\hline
\end{tabular}

ALCOA 62

ALCLA 17

baFnaC aLCOA 17

NONE ALCOA 17

LII 1

LII 1

LII 1

LII 1

LI 1

LI I 1

MONE LIIB

NONE LIT

4.05 .1935100

$4.6 \quad 5,1935160$

NOAE WIT 7

NCNE LIt

1179

UI 9

$-0.2+1.0$

LIT 9

i. 9

1.59

1.11 9

LII 9

1119 
TABLE II

MISCELLANEOUS CORAOEIDN INFORMATION

CREVICE

CREVICE

ADITIONAL SAMPLE DESCQTOTION

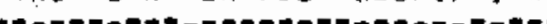
I DENT. METAL

CTAC

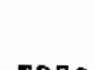

CHAMGE IN

PROPERTY

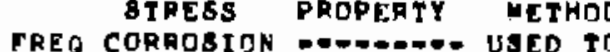

DTPTH PIT, COA.O.- TS EL MEASURE INCHES SOCM KBI T/M t PITS

SEA WATER EXPOBURE CONDITION

FHOW

TEMP O2 SAL CU2 COJ MARINE SEC PH (C) ML/L PFM PPH PPM GPOLTH PEFERENCE

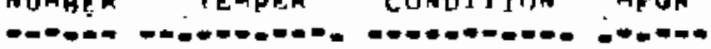

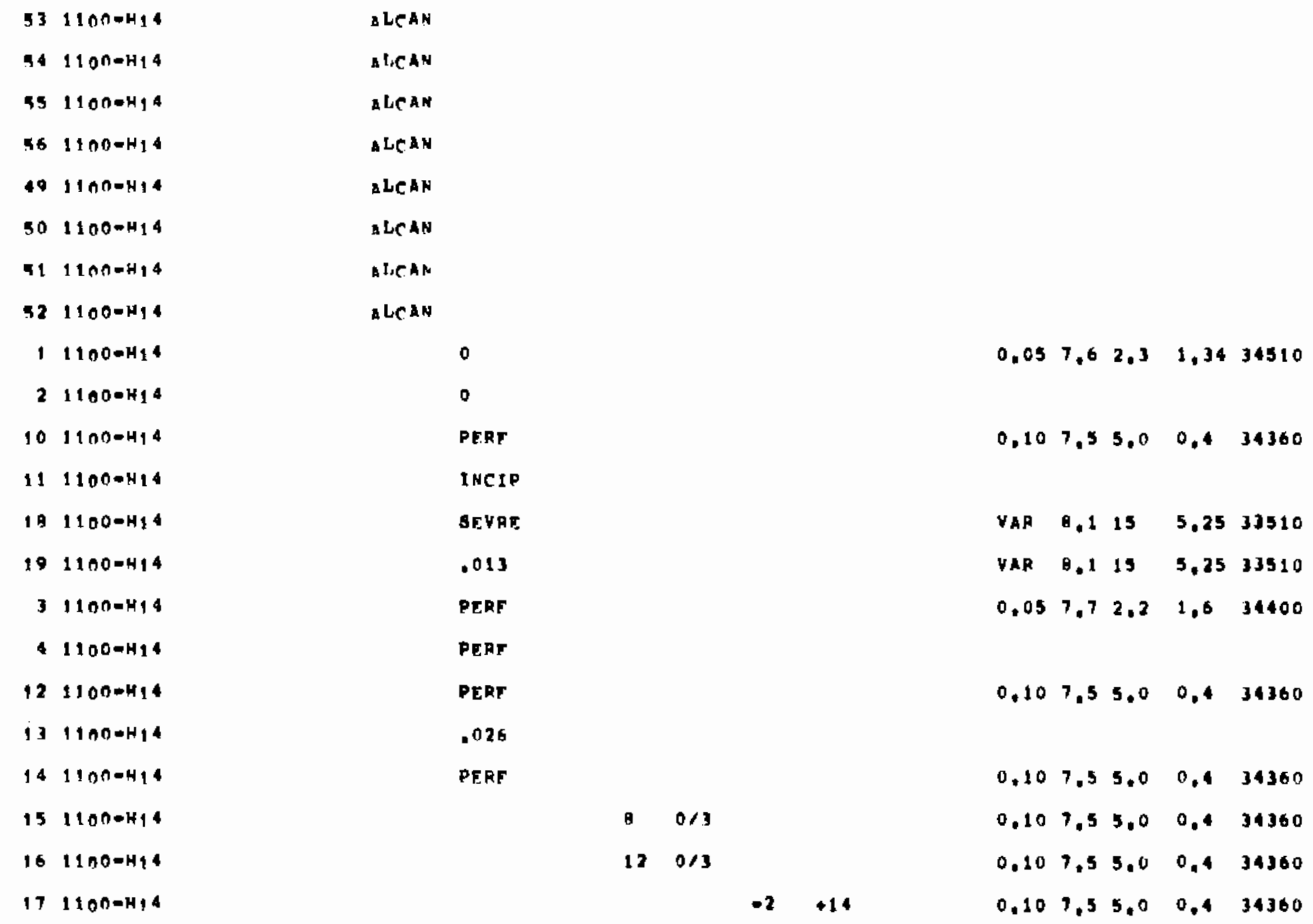

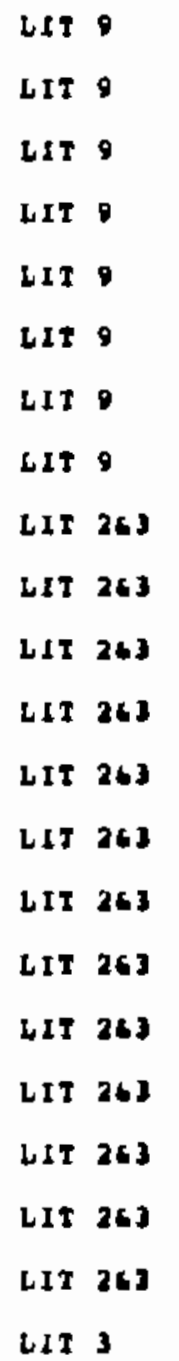


TAPLF II

MISCELLANERUS CRPROSION THFTFMATIUN

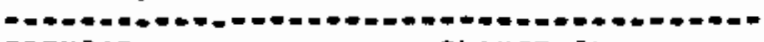
CRFVICF ATTACK STRESS PROPERTY METHOD

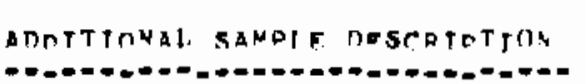

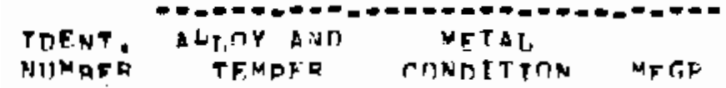

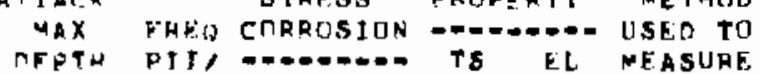

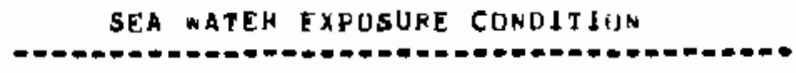

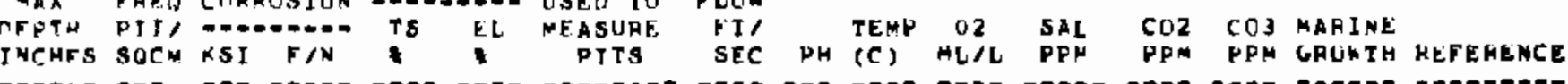
$\lim _{-\rightarrow 0}$ TFMPFE pits

\begin{tabular}{|c|c|}
\hline 5 & $11 n n=H+4$ \\
\hline h & $1+n n=4+4$ \\
\hline 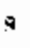 & $(1 \cap n=-4) 4$ \\
\hline 9 & $1900-414$ \\
\hline
\end{tabular}

1) $1160-H_{14}$

$31116 n-414$

3) $1160=14$

$33116 n=414$

72 1190.

$231190-$

24 112n-

In 1 and 1 A

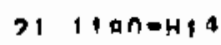

$251100=$

29 1190.

on 11000

$>011900$

771090

3011000

an 301130

a 2003-
NTIE FINISN aliena

aIti FIMISH alerta

"TIL FINISH ELCTA

"TLT FINISL ALEDA

OF Y

DFY

Prop

FERF

ACET RLFAN DFY

ACET CLEAN RFY

ACRT CLEAN DFY

ACFT CIFAM MFY

ACFT CIFARi FHY

ACFT CIEAN OFY
.1128

\section{PERF \\ PFPF \\ SEVRE}

DFRF

$\begin{array}{llll}2 & 0 \\ & & \\ 6 & 0 / 3 \\ 10 & 0 / 3\end{array}$

$-13-40$

UTRUSC

ICRISC

NICRUSC

MICRISSC

MtCrisc

MCRISC

HICROSC

$-2,1-14$ meansc

$-2.1-7.9 \mathrm{NICOOSC}$

$.05 \mathrm{DF}$
$0.107 .5 \quad 5.0 \quad 11.4 \quad 34360$

$0.107 .55 .0 \quad 1 \%, 43360$

$0.107 .55 .0 \quad 0.434360$

$0.107 .55 .00,434360$

$0.057 .02 .3 \quad 1.3 \quad 34510$

411763

$0.057 .52 .6 \quad 1.2 \quad 34510$

LII $2+3$

LII $2+3$

LIT 263

LIT 243

ALCUA 62

ALCOA 17

BARMAC ALCOA IT

MONE ALCUA 17

LIT 263

L11 243

LII 3

LIT 243

[IT 26]

LIT 4

LII 4

LII

LIT

LIT

LIT 4

Lit 243

LIT 243 
TAPLE II

MISCELLANEOUS CORAOSION INFORMATION

CREVICE

CREVICE ETRESS CHANGE IH

ADNITIONAL SAMPLE OFSCRIPTION

ATTACK

PROPERTY METHOD TDENT. ALLOY AMD NOTAL
NUMBFR TEMPER CONDITION MFGR

MAX FREO CORROSION -...... USED TO DEPTH PIT, ....... TS EL MEASURE INCHES SOCM KSI F/N GEA WATEA EXPQSUAE CONDITION
FLOW

TEMPER. CONDITTON MFGR

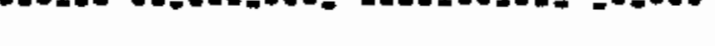
Q3 30n3-
A5 3003 -
$943003-$
95 30n3-
7030030
12 3no 3*
$97 \operatorname{son} 3$ *
ค9 30030
$743003=$
$763003-$
78 3nn3-
RO 30030

$1133003-F$

$1153003-5$

$2133003-5$

$2123003-5$

$1163003=\mathrm{H}^{4}$

$1213003 \times 414$

$2253 n n 3-414$

$2263 n_{0} 3-H_{1} 4$

$2.730 \cap 3-414$

$1253003=H_{14}$
.040

INCIP

$.05 \mathrm{PF}$

.066

.040 PF

.050 PF

$.040 \mathrm{DF}$

$.040 \mathrm{PF}$

.05 PF

A LCOA

a LCOA

MACHINET MCDA

HILE FIMISH ALCOA

at,eos

$\operatorname{arcon}$

$-0.2-1.5$
$0.107 .55 .0 \quad 0.4 \quad 34360$

VAP $0.115 \quad 5,25 \quad 33510$

VAR $0.1 \quad 15 \quad 5.25 \quad 33510$

$0.057 .72 .2 \quad 1.6 \quad 34400$

$0.107,53.0 \quad 0.434360$

$0.057 .62 .3 \quad 1.3 \quad 34510$

$0.05 \quad 7,5 \quad 2,6 \quad 1,2 \quad 34510$

$x-\operatorname{sect}$

$x+8 \operatorname{ect}$

$4.65 .18 \quad 35160$

$4.65 .18 \quad 35160$
L1T 263

L[T 26)

LIT 243

LIT 243

LIT 243

LIT 26$)$

$412 \quad 263$

LI 263

LIT 263

LIT 263

LIT 263

LIT 263

ALCOA 24

ALCOA 24

ALCOA 7

ALCOA 7

ALCOA 3

ALCOA 3

LIT 9

LIT 9

LIT 9

ALCOA 15 
TAHIE II

MISCEILANECISS CORACSIMN INFORMATION

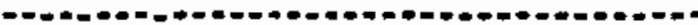

CREVICE

CREVICE
ATTACK SHANGE IN
STRESS PROPFATY METHOD

ATITTITHAL SA WPLE TIESCETOTITN

STRESS

ROPFATY METHOD

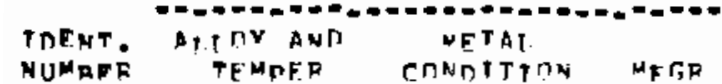

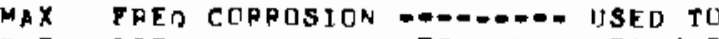

DEPTH PIT, -...-.... TS EL VEISIRE

SEA WATEH EXPOSURE CONDITION

INCHFS SDCN KSI F/N

3 PITS

FLOW

0N

FT' TEMP O2 5AL CU2 CUJ MAHINE

-

INCHe

SEC PH (C) ML/L PPM PPH PPM GALITH PEPERLNCE

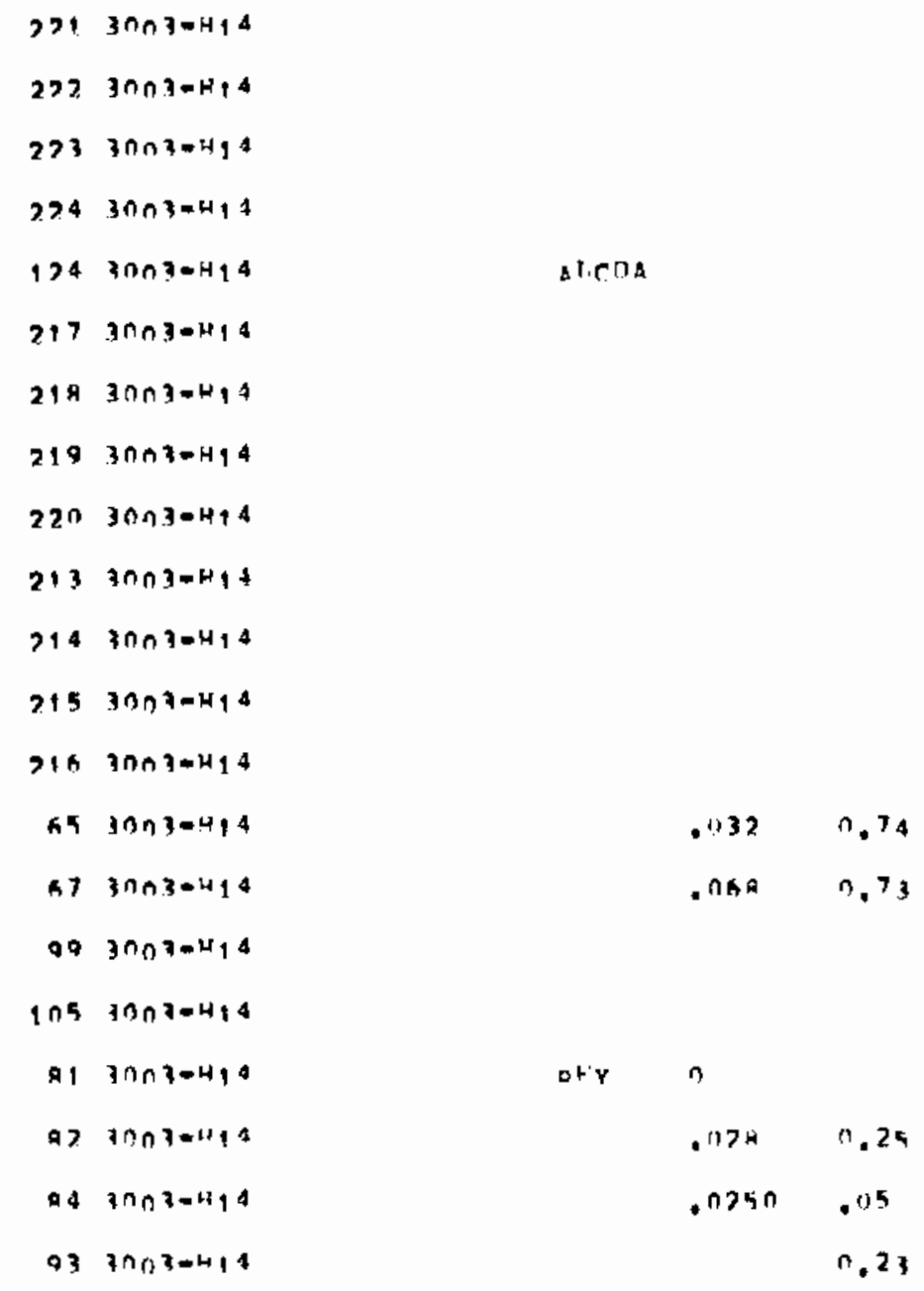

$-3$

0.50

$0.05 \quad 7.0 \quad 2.3 \quad 1.3 \quad 34510$

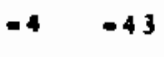

$+4-1$

104 $3(2 n)=414$
0.23

$-6+15$
LIT 9

L. I 19

L1T $y$

LIT 9

ALCOA 14

LII 9

LIT 9

LIT 9

LII 9

LII 9

LIT 9

LII 9

LII 9

617263

L11 253

LIT 263

LII 253

LII 3

LIT 26]

(1I 263

LIT 2+3

$411 \quad 263$ 
TABLE II

MISCELLANEOUS CORROSION INTORMATTON

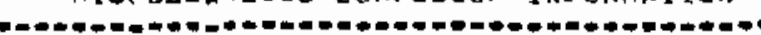
CPEVICE

CREVICE CHAMGE IA

STRESS PROPERTY METHOD
MTTCK
MAX FREO CORROSION

ADATTINNAL SAMPLE DFSCRTDTJON

DEPTH PIT,

INCHES SOCM KSI F/N

SEA WATEA EXPOSURE CONDITIOH
FLOH
FT/
SEC PH (C) ML/L PPM PPM PPM GROWTH RETERENCE

NUMAER TEMPE CONDITTON MFGR

PITB

- PH (C) ML/L PPM PPM PPM GROWTH REFEREHCE

$69 \quad 1003-\mathrm{H}_{1} 4$

iI $3003-\mathrm{H}_{14}$

A. $3003 \div 414$

A $3003-\mathrm{H}_{14}$

$00 \quad 3003=H_{14}$

$913 \cap \cap 3=\mathrm{H}_{1} 4$

$923003=4,4$

Q6 $3003-H_{14}$

$973003-\mathrm{H}_{1} 4$

$1003003 \div \mathrm{H} 94$

ดค 3กด 3-1 14

$733003 * \mathrm{H}_{1} 4$

$753003-414$

$1513003-\mathrm{H}_{14}$

$1023003-\mathrm{H}_{1} 4$

$73003-\mathrm{H}_{1} 4$

$793003=H_{14}$

$1033007=\mathrm{H}_{1} 4$

$2073 \mathrm{OO}^{2} \mathrm{OH} \& 4$

2กค $3003-\mathrm{H}_{14}$

$2053003-H_{14}$

$20630 n 3 \cdot H_{14}$

$\begin{array}{llll}.066 & 0.06 & \\ .052 & 0.05 & \\ .093 & & & \\ .0701 & .02 & & \\ & & 6 & 0 / 3 \\ & & 9 & 013\end{array}$

$46 \quad+5$

0

.0731

0

$.125 \mathrm{PF} .13$

.125 PF .05

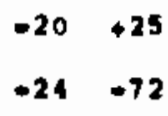

$.125 \mathrm{PF} .08$

$-3 \quad-20$

$0 \quad-20 \quad x=S E C T$

$4 \quad-14 \quad x-8 E C T$

$0 \quad+0 \quad x=\operatorname{sect}$

$+1+15 \quad x=5 E C T$
$0.05 \quad 7.7 \quad 2.2 \quad 1.6 \quad 34400$

LIT 263

LIT 263

$0.107 .55 .0 \quad 0.4 \quad 34360$

LIT 263

LIT 263

$0.107 .55 .0 \quad 0.4 \quad 34360$

LIT 24]

$0.107 .55 .0 \quad 0.434360$

LIT 263

0.107 .55 .00 .434360

LIT 3

VAP $8.115 \quad 5.2533510$

LIT 203

YAR $8,1 \quad 15 \quad 5,2533510$

LIT 263

$0.057 .72 .2 \quad 1.6 \quad 34400$

LIT 263

VAค $8.115 \quad 5,2533510$

LIT 243

$0.057 .6 \quad 2.3 \quad 1.3 \quad 34510$

217263

LIT 26)

$0.057 .62 .3 \quad 1.3 \quad 34310$

LIT 263

2IT 243

LIT 283

LIT 2 G

L1T 283

ALCOA 11

a LCOA 11

ALCUA 11

ALCoA 11 
TAALE II

MTSCFLLANFOUS CORRESTON INFCRMATTUN

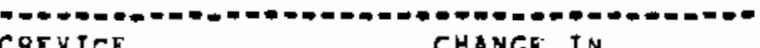

catidit

STRESS PHANEETTY IN METHAD

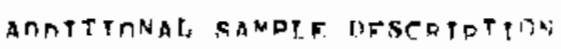
TACK

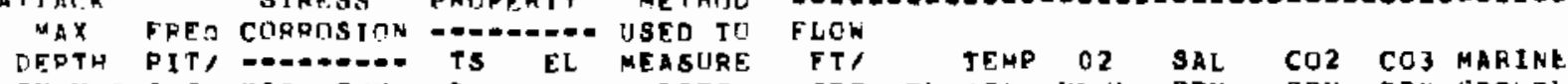

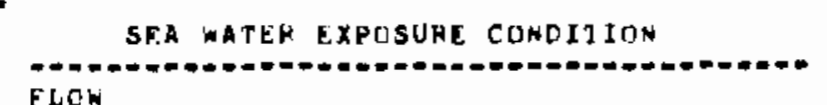

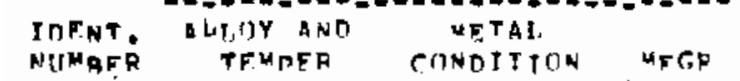
INCHFS SOCM KSI F/N PITS SFC PH CC ML ML PPM PPH PPH GROWTH

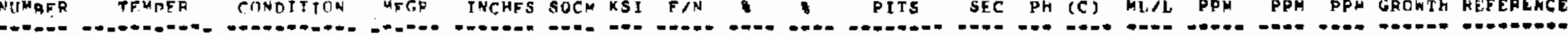

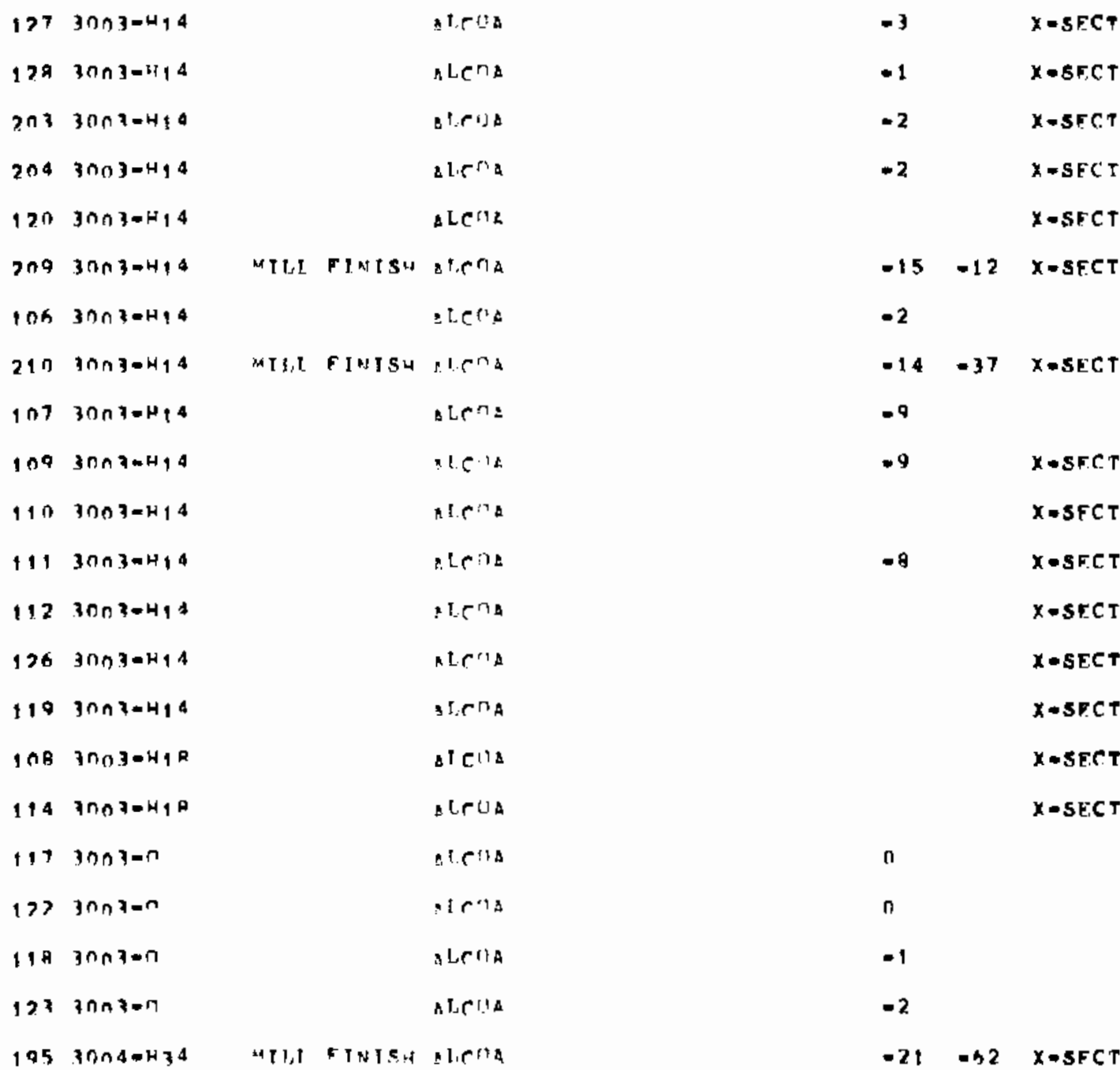

ALCUA 12

ALCUA 12

ALCOA 12

ALCUA 4

$A L C O A 65$

ALCOA 41

ALCOA 21

ALCUA 4

ALCOA 21

ALCOA 22

ALCUA 21

ALCOA 22

ALCOA 22

ALCUA 50

ALCOA 36

ALCOA 64

ALCUA 64

ALCOA 3

ALCUA 3

ALCOA 3

A LCUA 3

ALCOA \&1 
TABLE II

MISCTLLANEOUS CORROSION INFORMATION

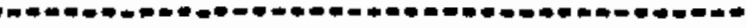

CREVICE

CREVICE

STRESS CHANGE IN

STRESS PROPERTY METHOD
PREO CORROSION

ADNITTONAL SAMPLE DESCAIPTION

TACK

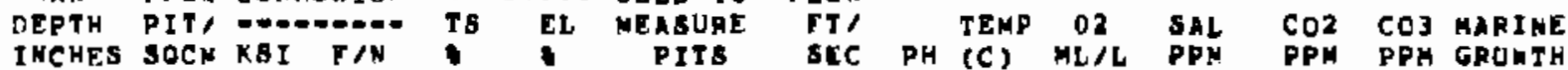

SEA WATER EXPOSURE CONDITION

IDENT. ALLOY AND METAL NUMBER TEMPER. CONDITTON MFGR

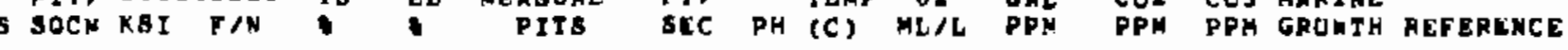

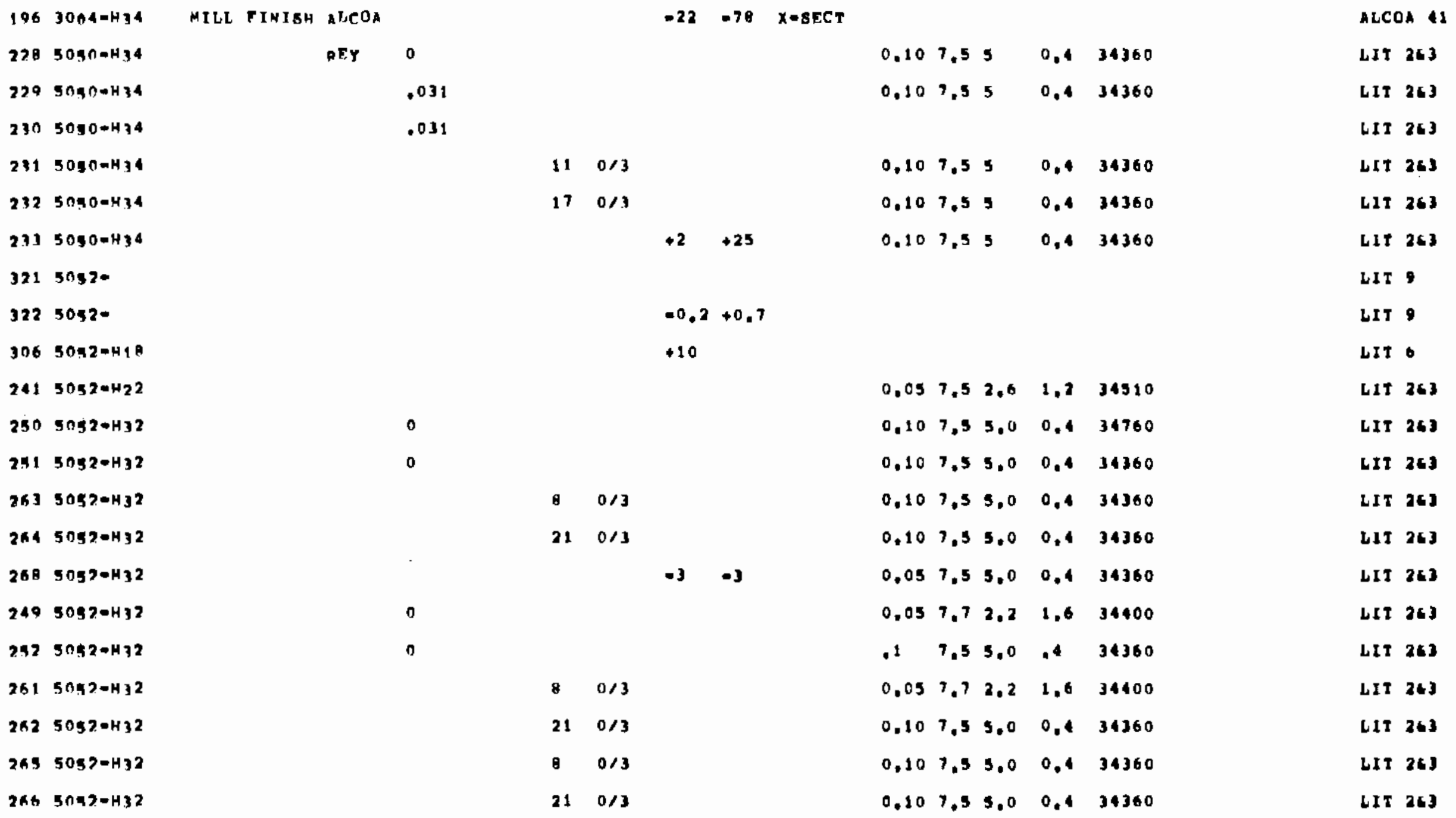


TAELF II

MISCELLANEUS CORRCSIUN TNFORMATIJN

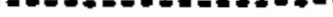
COEVICF. CHANCE IN ATTACY STRFSS PROPERTY METMOIO MAX FQEO CIIRRUSITN -..2...- IISTD TO

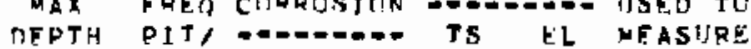
INCHFS SUCM KSI F/N TS EL MASITRE

ADNITIONAL SAMPL.F. DFSCLTOT TDENT.

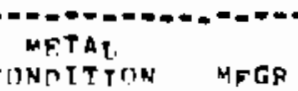

1 PTIS

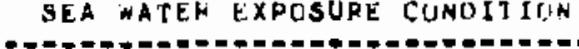 \\ SEA WATEH EXPOSURE CUNOITITIN FLUW TEMP O2 SAL CIIZ CO3 MARIME SEC PH (C) MLLL FPM PPM PPM GFCAIH KEFEHENCE}

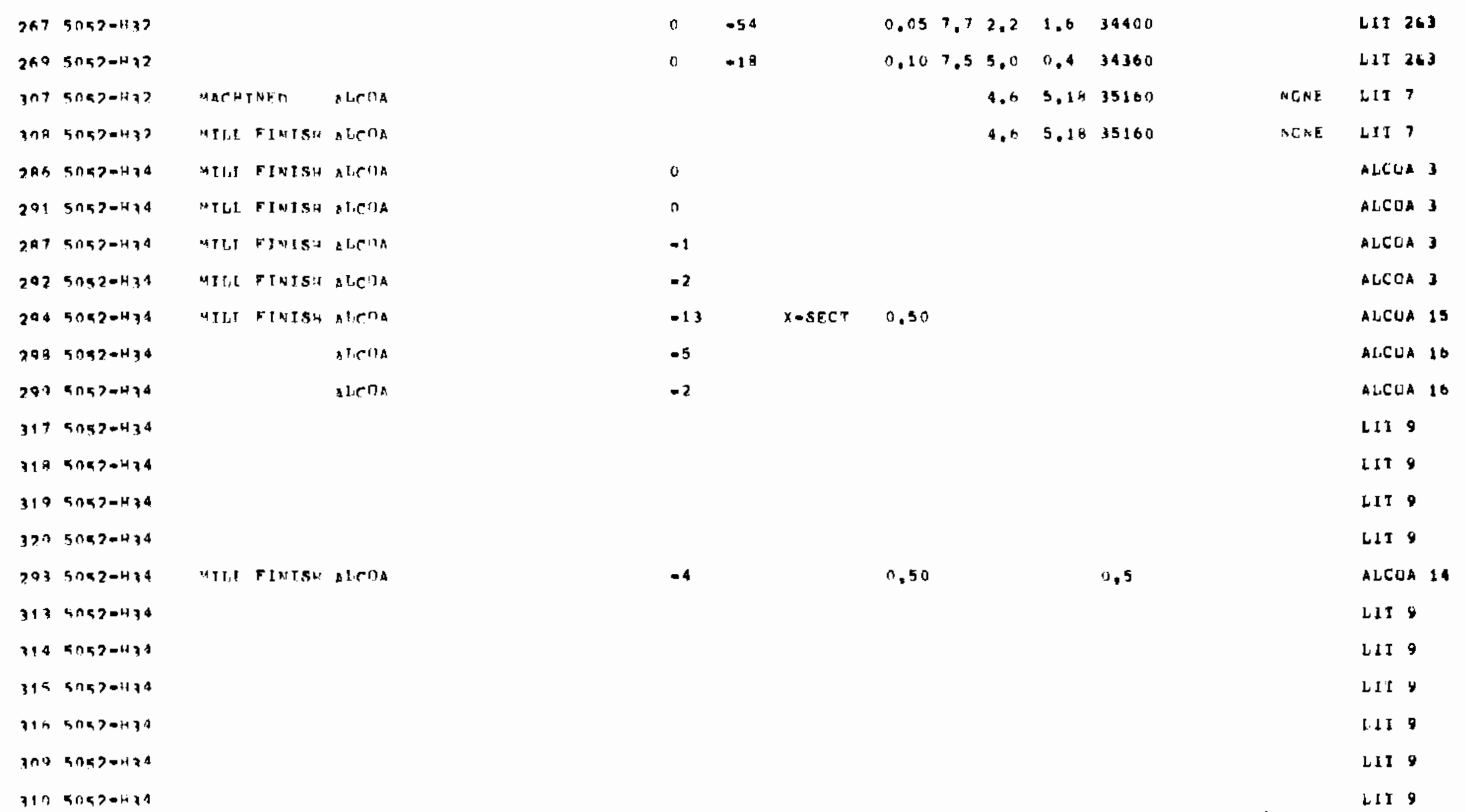


TABLE II

MIBCELLANEUUS CORROSION INTORMATION CREVICE CHAMGE IM

ATTACK BTRESS PROPERTY METHOD

MAX PRER CORROBION DAPE.A. USED DEPTH PIT/ TS EL MEASURE

ADATTIOHAL SAMPLE DESCRIPTION

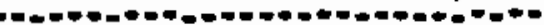
INCHES SOCM KSI TIM E PITS IOENT. ALLOYAND NETAL
NUMBFR TEMPER CONDITYON MFGR

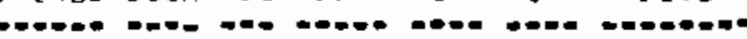

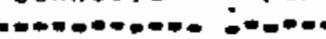

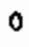

MOD

$2545052-434$

$2705092-439$

$250 \operatorname{sos} 2-\mathrm{H}_{3} 4$

25950520 H 39

$2605052=434$

$2725052-H_{3} 4$

$2955092-139$

$2565052-1334$

$2575052-\mathrm{H}_{34}$

$2715052=434$

$27350520 \mathrm{H}_{34}$

$274 \operatorname{sos} 2 \cdot H_{3} 4$

$275 \mathrm{SOg}_{2}=\mathrm{H}_{3} 4$

$2095052-H_{3} 4$

$2765052-\mathrm{H}_{3} 4$

$2775052+434$

$2785052-\mathrm{H}_{34}$

$27950,2-\mathrm{H}_{34}$

$3 \mathrm{nO}^{2} 5052-\mathrm{H} 34$

MILL FINISH ALCOA

MILL FINISH aLCOA

MILL FINISH ALCOA

MILL FINISH BLCOA

MILI FINISH ALCOA

MILT. FINISH ALCOA

MTLL FIMIBH aLCOA

MILL FINIGH ALCOA

ALCOA

mOD
0
.034
.050
0
MOD
SLIGT

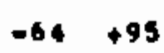
FI/ TEMP O2 SAL CO2 COJ MARINE

SEA WATER EXPOBURE CDNDITION

LOW

SIC PH (C) ML/L PPM PPH PPM GRDWTH REFEREMCE
0

o

$-4$

$\mathrm{X-sECT}$

$0.05 \quad 7.62 .3 \quad 1.3 \quad 34510$

$0.057 .6 \quad 2.3 \quad 1.3 \quad 34510$

0.057 .62 .31 .334510

$0.057 .55 .0 \quad 0.4 \quad 34360$

$0.057 .5 \$ 000.4 \quad 34360$

$0.107 .35 .0 \quad 0.434360$

$0,057,62,3 \quad 1,3 \quad 34510$

$0.057 .62 .3 \quad 1,3 \quad 34510$

$0.057 .62 .3 \quad 1.3 \quad 34510$

$\mathrm{x}-\mathrm{sect}$

0

$-1$

$-7$

$-1$

$-2$
LII

LIT 9

LIT 243

4IT 24$\}$

LIT 26 J

LIT 243

LIT 2G3

C17 263

LIT 2G3

LIT 263

LIT 263

LIT 243

GIT 263

ALCOA 19

ALCOA 7

ALCOA 5

ALCOA 65

ALCOA 7

ASCOA 22

ALCOA 21

ALCOA

AsCOA 16 
TARIF II

MISCFLI ANFOUS COPACSION TNFOAMATIUN

CBFVICF,

$\begin{array}{lll}\text { CRFVICF: CHANGE IN } & \\ \text { ATTACY STRFSS PAOPERTY METHOI? }\end{array}$

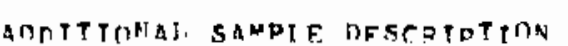
STRFSS PFOPERTY

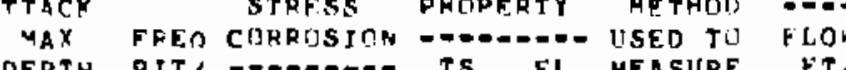

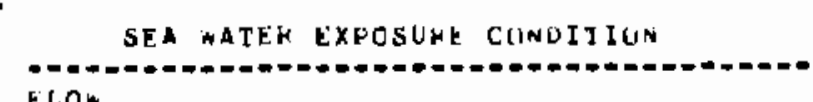

DFPTH PIT, TS EL MFASUPE HT, TENP O2 SAL COZ COBMARINE INCHFS SOCN KSI F/N

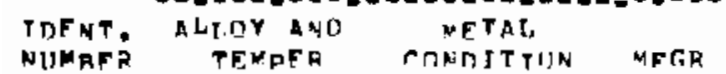
HEC DH (C) ML/L PPM PPA PPM GROMTH REFERENCE

\begin{tabular}{|c|c|c|c|c|c|c|c|}
\hline 301 & $50 \times 2-434$ & & & $A t_{1}, 0 \mathrm{~A}$ & & -1 & \\
\hline 290 & $5052-4 \times 4$ & $4 \mathrm{TL}$ & FINIS4 & $A \operatorname{LrOA}$ & & -6 & $x-\operatorname{sect}$ \\
\hline 291 & $50 \times 2-434$ & $M T I I$ & FI N I SH & $\triangle \mathrm{LCOA}$ & & & $x=5 F$ \\
\hline 2ค2 & $5 \cap 52-H_{3} 4$ & $M I L L$ & F I N I I S H & $x \operatorname{lon}$ & & -5 & $x-5 \mathrm{EC}$ \\
\hline 293 & $5052-H 34$ & MTLI & FINISH & $A C D A$ & & & $x=S E$ \\
\hline $2 R 4$ & $5 \cap 52-4 \times 4$ & זוזו & FIMISY & $\operatorname{IsC} \mathrm{CA}_{\mathrm{A}}$ & & -1 & $x=\mathrm{SEC}$ \\
\hline $3 \cap 2$ & $5 \cap 52-434$ & & & Al.ritA & & -4 & \\
\hline $\ln ?$ & $5052-H 34$ & & & $x \operatorname{tac} 1 \mathrm{~A}$ & & -3 & \\
\hline 296 & $5057-434$ & & & $\operatorname{sen} A$ & & +1 & \\
\hline 297 & $5 n 52-4 \times 4$ & & & $A C \cap A$ & & -4 & \\
\hline $\ln 4$ & $5057-H_{3}^{4}$ & & & ALCOA & & -2 & \\
\hline 305 & $5057-434$ & & & 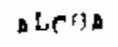 & & -1 & \\
\hline 20月 & $5 \cap 52-H 34$ & & & ALCOA & & & $x=\sec$ \\
\hline 295 & $5092-434$ & & & $A I C \cap A$ & & & $x=55$ \\
\hline 295 & $5 n 52=8$ & $a_{1,1}$ & $F[N i 15 H$ & $A I C D A$ & & 0 & \\
\hline 200 & $50<2=7$ & पILI & F IN T SH & $\triangle L C O A$ & & 0 & \\
\hline 214 & $5057=n$ & & & & PERF & & \\
\hline 235 & $5 n \times 2=n$ & & & & PFAF & & \\
\hline 243 & $5052=11$ & & & & PFPF & & \\
\hline 244 & $5 n 52-n$ & & & & Jicicte & & \\
\hline 247 & $5052-1)$ & & & & 0 & & \\
\hline $24 R$ & $5 n<>-11$ & & & & . & & \\
\hline
\end{tabular}

VAF $8.1 \quad 15 \quad 5.2533510$

ALCOA 16

ALCOA 21

ALCOA 21

ALCUA 22

ALGUA 22

ALCOA 2

ALCOA 16

ALCOA 1 o

ALCLA 10

ALCOA 10

ALCOA 10

ALCOA 16

ALCOA 56

ALCUA 56

ALCOA 3

ALCUA 3

LIT 243

LIT 2t+3

LII 24J

LIT 2t3

LIT 263

LIT 263 
TABLE IT

MISCELLANEQUS CORROSION INFORMATION

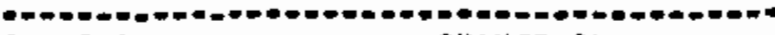

CREVICE

CREVICE

STRESS PHANGE IN

ADATTITHAL SAMPLF. DFSCRTPTIDN

ATTACK STRESS PRDPERTY METHOD

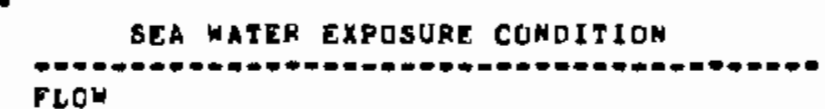

DEPTH PIT/

INCHES SOCM KSI FIN

$\begin{array}{lllll}\text { FI' TEMP } 02 & \text { SAL } & \text { CO2 } & \text { COJ MARINE } \\ \text { SEC PH CC) ML/L PPM PPM } & \text { PPM GPONTH }\end{array}$

IDENT, ALLOY AND METAT.
NUMBTR TEMPER CONDITTON MTGR PIT

\begin{tabular}{|c|c|c|c|c|}
\hline 216 & $5042=0$ & & & \\
\hline 217 & $5052=n$ & & & \\
\hline 245 & $5 n 52=n$ & & & \\
\hline 246 & $5052=n$ & & & \\
\hline 238 & $5052=n$ & & & \\
\hline 239 & $3052-0$ & & & \\
\hline 240 & $5052=n$ & & & \\
\hline 242 & $5052-n$ & & & \\
\hline 328 & $3052=0$ & MTLL. & FINISH & AlCOA \\
\hline 329 & $\sin 32=\pi$ & WTLI & FINISH & ALena \\
\hline 330 & $5042=0$ & MILI & FINISH & ALCOA \\
\hline 331 & ח- & MILL & FINISH & ALCOA \\
\hline 332 & $5052=n$ & & FINISH & \\
\hline 323 & $5052-0$ & MILI. & FINISH & ACOA \\
\hline 324 & $5052=n$ & M I LI. & FIय ISH & $\triangle L C O A$ \\
\hline 325 & $5052=0$ & MILL & FINISH & aLCOA \\
\hline 326 & $5052=n$ & MILL & FINISH & $\triangle \mathrm{LCOA}$ \\
\hline 327 & $3052=n$ & $M Y L L$ & FINISH & ALCחA \\
\hline 378 & 50560 & & & ALCAN \\
\hline & 305 & & & \\
\hline
\end{tabular}

PERF

PERF

.020

0

PERF

PERF

PEAF

Pr.RF

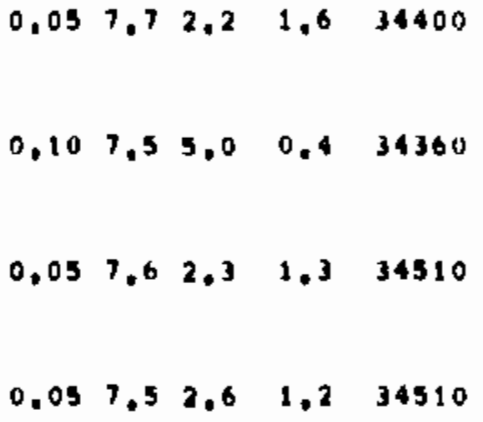

LII 263

LIT 263

LIT 263

415 263

LIt 263

LIT 263

[IT 263

LIT 24]

BARMAC ALCOA 37

BARMAC ALCOA 3

BARAAC ALCOA 37

GARNAC ALCOA JT

BARNAC ALCOA 37

ALCOA 37

BARNAC ALCOA JT

GARNAC ALCOA 3T

BARMAC ALCOA 37

GARMAC ALCOA 37

LIT 9

LIT 9

4129

LIT 
TALE II

MPSELLANEQUS CORRCSION INFORMATTUN

\begin{tabular}{|c|c|c|c|c|c|c|c|c|c|c|c|c|c|}
\hline DFVICE. & & & CHANGE IN & & & $\operatorname{SEA}$ & WATER & $E X P C$ & SURE & UNOI & Gen & & \\
\hline TTACK & & STAFSS & PROPERTY & HETHOD & . & & $-\infty=-0$ & - - & $=-\infty$ & 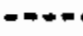 & 4 & & \\
\hline$M A X$ & FRED & COARUSION & $\because-\infty+\infty \cdots$ & USED TO & F LOW & & & & & & & & \\
\hline $\begin{array}{l}\text { NFDTH } \\
\text { INCHFS }\end{array}$ & $\begin{array}{l}\text { PIT' } \\
\text { SOCM }\end{array}$ & KSI $F / N$ & TS & $\begin{array}{c}\text { MEASURE } \\
\text { PITS }\end{array}$ & $\begin{array}{l}\text { FI' } \\
\text { SFC }\end{array}$ & $\mathrm{PH}$ & $\begin{array}{l}\text { TEMP } \\
\text { (C) }\end{array}$ & $\begin{array}{c}02 \\
M L L L\end{array}$ & $\begin{array}{l}\text { SAL } \\
\text { PPH }\end{array}$ & $\begin{array}{l}\text { CU2 } \\
\text { PPA }\end{array}$ & $\begin{array}{l}C 03 \\
\text { PPS }\end{array}$ & $\begin{array}{l}\text { MAFINE } \\
\text { GROWTH }\end{array}$ & YCE \\
\hline
\end{tabular}

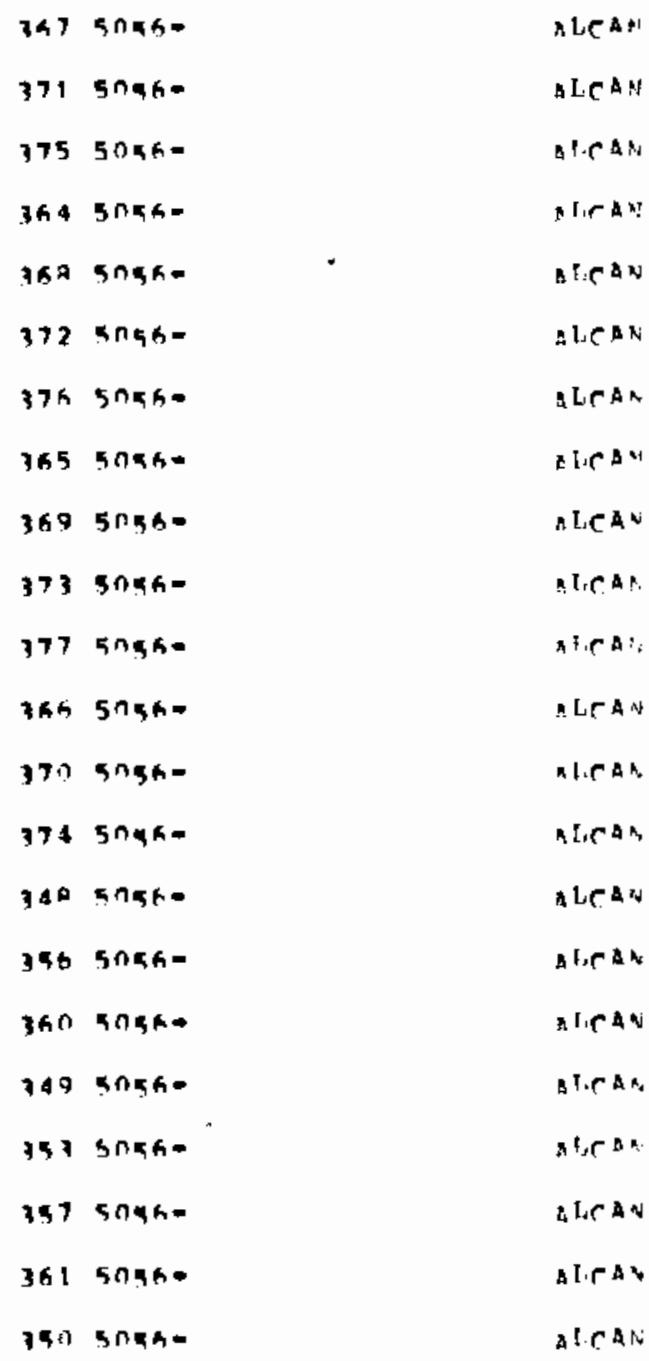


TABIE II

MISCELLANEUUS CORACSION INFOAMATIDN

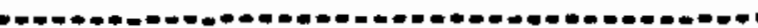
CAEVICE. CHANGE IN SAEVICE
ATTAK ADOTTINNAL SAMPIE DESCRTOTTON -

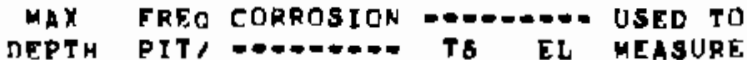
BEA WATEH EXPOSURE CONDITION
FLOW

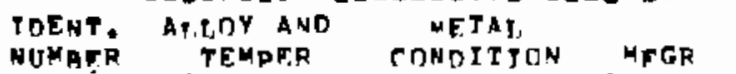
TEMP 02 SAL CO2 CO3 MAHIN INCHES SOCN KSI F/N 2 PITS SEC PH (C) ML/L PPM PPM PPM GROHTH RETEHLNCE

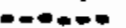

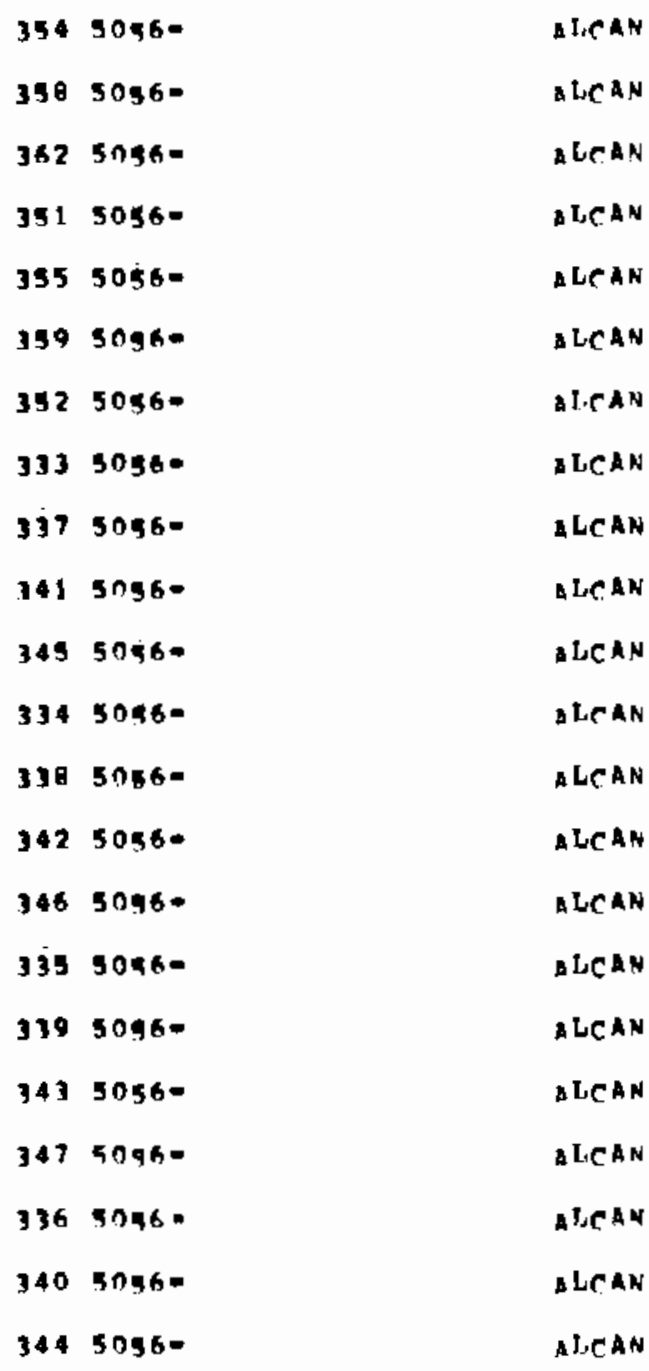

LI T

LIT

LIT

LIT 9

LI 9

LII

LIT 9

LII

2II 9

LIT

LI

LIT

LIT

LIT

LIT

LIT 9

L1T 9

LII

LIT

LIT

LIT 9

LIT 
TAPLE II

MISCELLANEUUS CORRCSION INFGHMATION

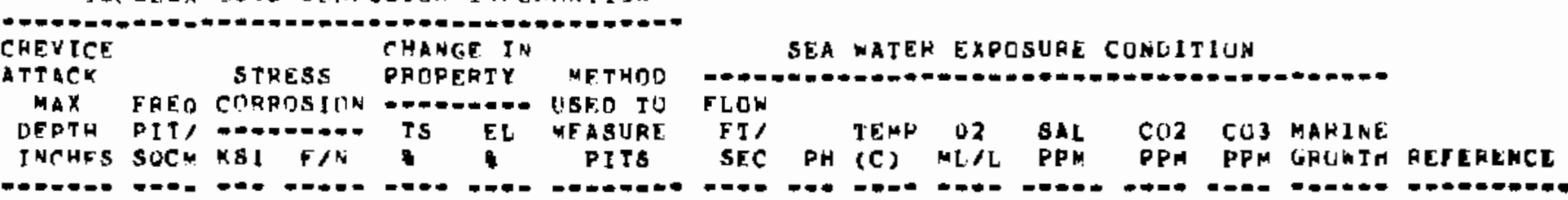

$4+05093=$

LII 9

4115กด3-

$+0.1=0.5$

[1]

$-0,7-2,3$

II 9

$\operatorname{LIT} 9$

$\triangle \operatorname{LCAN}$

LII 9

LI 19

$\triangle \operatorname{tic} A N$

I.15 9

ILCAN

LIT

ALCAN

LIT

A.CAN

LII 9

aICAN

II 9

alic 4 |l

III 9

a LCAN

LII 9

$\operatorname{ACAN}$

LIT 9

$A L C A N$

LII

a pic a

LIT

ATSAN

LIT 9

$\sin a$

LIT 9

ALTAN

LIT 9

A TISAR:

LII 9

$\triangle \mathrm{LCAN}$

LII 9

a ISTAN 
TABLE II

M ISCFLLANEOUS CORRCSION INFORMATION

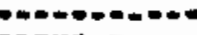
CAEVICE CHANGE IN

ATTACK STRESS PROPERTY METHOD

MAX FREO CORROEION - -

AMAITIMNAL SAMPTE DESCDTDTTON DEPTH PIT, =...... TS EL MEABURF.

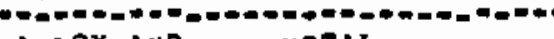
IDENT. ALLOY AND METAL
HUMBR TEMPE CONDTTON MFGP INCHES SOCM KSI F/N

BEA WATER EXPOSURE CONDITION

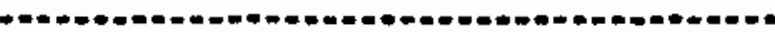
FIOW TEMP 02 SAL CO2 COJ MAKIME SEC PH (C) ML/L PPH PPM PPM GROHTH RETEHENCE

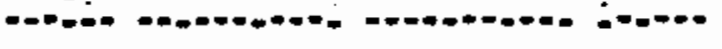

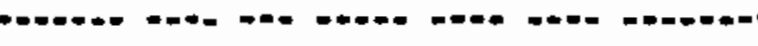

SEC-

\begin{tabular}{|c|c|}
\hline 1106 & 50830 \\
\hline 1110 & SOR3 \\
\hline 475 & $5003=$ \\
\hline 1006 & $5093 *$ \\
\hline 1100 & 50ค3- \\
\hline 476 & $5093=$ \\
\hline 1097 & $5003-$ \\
\hline 1101 & $5013=$ \\
\hline 1094 & $5093-$ \\
\hline 1098 & $5093=$ \\
\hline $1 \ln 2$ & 5093- \\
\hline 1095 & $5003=$ \\
\hline 1099 & \\
\hline
\end{tabular}

LI

LIT

LIT

LII 9

LIT 9

LIT 9

LII 9

LIT 9

LIT

LIT

LIT 9

LIT 9

LI I

4IT

VAR $0.1 \quad 15 \quad 3,2533310$

LIT 243

43550 3.

LIt 343

0

[1T 26]

158 5093-H:13 ACET CLFAN DEY

MICAOSC

LIT 4

462 50Q30H113 ACET CLFAN PEY

MICAOSC 
TAPLF II

MISCELLANEOUS CURPCSION TRFTRMATION

ARATTIONAI SAMDFE DFSCRTOTITIN

ATTACK

STHESS HROPERTY

METHOO

NAX FFEQ CIRFISION - ...... HSED TO

DEDTH OIT, CS EL MFASUPE

INCHFS SOCK KEI F/N IS

PITE TIJ TEMP OZ SAL COZ COJ MARINE

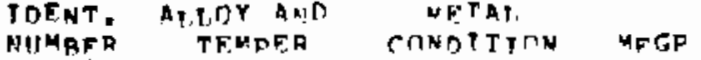

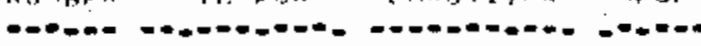

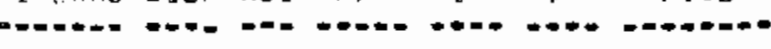

\begin{tabular}{|c|c|c|c|c|c|c|}
\hline 445 & $5093-4113$ & & & & +4 & +26 \\
\hline $4: 5$ & $5 \cap 93-H_{1}+3$ & INCIP & & & & \\
\hline 416 & $5 O R+-H_{1} 13$ & .0 .3 & & & & \\
\hline 417 & 50 a $3-\mathrm{H}, 13$ & .11 & & & & \\
\hline 418 & 50 月 $3-\mathrm{H}, 13$ & $n$ & & & & \\
\hline 494 & $5 \cap 03=4113$ & $n$ & & & & \\
\hline 425 & $5 \cap A 3=H_{1} 13$ & $n$ & $n .11$ & & & \\
\hline 426 & $5 \cap 9\}=H+1\}$ & $n$ & & & & \\
\hline 427 & $5 \cap 93=4,13$ & 0 & 0.03 & & & \\
\hline 434 & $5 \cap 03-H\{1\}$ & .0030 & & & & \\
\hline 4 ib & $5 \cap 83-H_{1} 13$ & 0 & & & & \\
\hline 444 & $5 \cap R 3=4113$ & & 15 & $0 / 3$ & & \\
\hline 449 & $5 \cap \times 3-H_{1} 13$ & & & & -1 & +37 \\
\hline $45 \pi$ & SIR $3=H+13$ & & & & +6 & .21 \\
\hline 453 & $5093-H_{1}+3$ & & & & -2 & +15 \\
\hline 454 & $5093-\mathrm{H}_{1} 13$ & & & & +9 & $+R$ \\
\hline 110 & $5 n \times 3=4,13$ & Ineto & 0.19 & & & \\
\hline 420 & $5 \cap B_{3}-H_{1} 13$ & ก & 0.37 & & & \\
\hline 421 & $5 \cap a 3-\omega_{1} 13$ & $n$ & 0.93 & & & \\
\hline $4>2$ & $5 \cap B_{3}=H_{1} 13$ & $n$ & $n .1 n$ & & & \\
\hline $42 B$ & $50 A 3=H_{1} 13$ & .052 & & & & \\
\hline $43 n$ & $5 \cap 03=4,13$ & 0 & .05 & & & \\
\hline
\end{tabular}

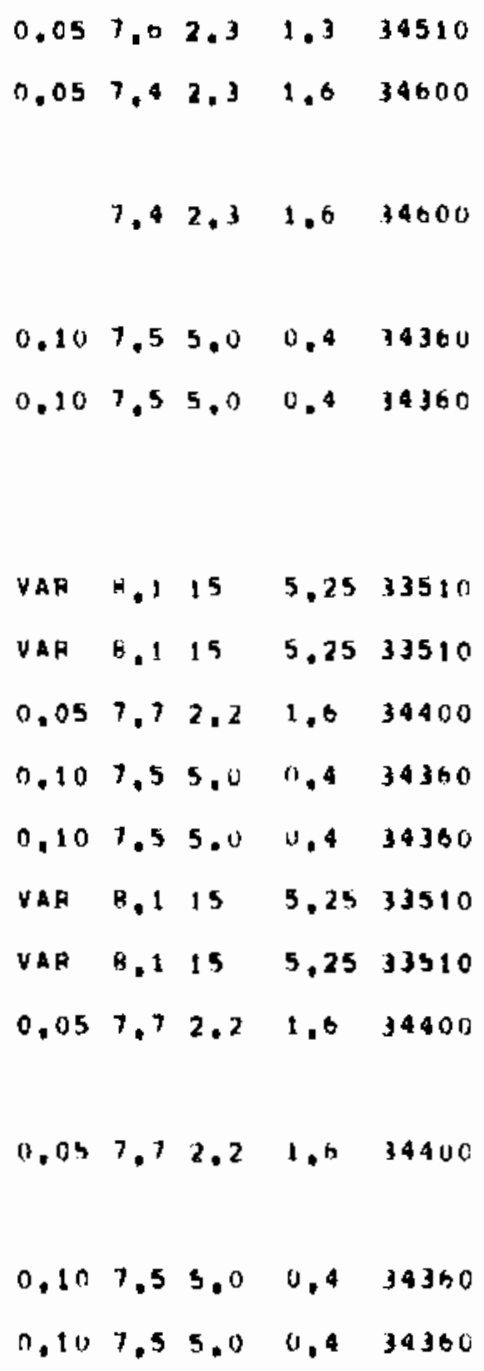

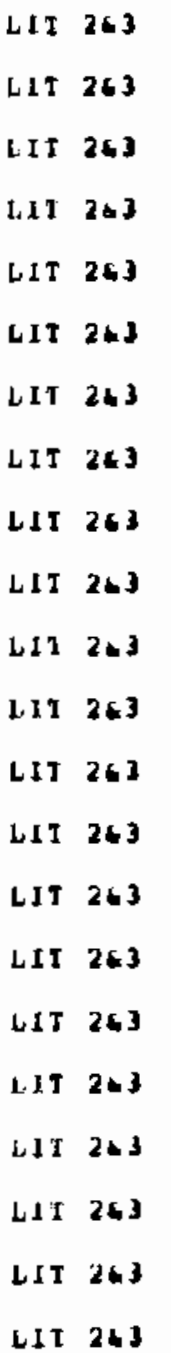


TABLE II

MISCELLANEOUS CORRESTON INFORMATIUN

CREVICE CHANGE IN

$\begin{array}{lll}\text { CREVICE } & \text { CHANGE IN } \\ \text { ATTACK } & \text { PTAESS PROPERTY METHOD }\end{array}$

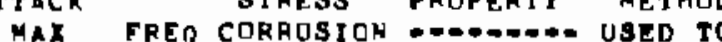

DEPTH PIT, TS EL MEASUPE INCHFS SOCM KSI F/N PITS

BEA WATER EXPOBURE CONDITIUN

AMATITNAL, SAMPIE DESCRTPT T

STS IEMP O2 SAL CO2 COJ MARIM

NOENTE ALLOY AND TERER CONDITTON MFGR

-..- PH (C) ML/L PPM PPH

PPM GROWTH REFEREMCE

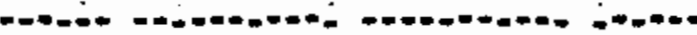

.0571

0

$43350,3-\mathrm{H}, 13$

41850 30. 113

$4395003-4113$

$4405 n=3-H_{1} 13$

$44150030 H_{13}$

$4425023-\mathrm{H}_{113}$

$4435013-\mathrm{H}_{113}$

$44650 \mathrm{~A}+\mathrm{H}_{113}$

447 5n:3-H113

451 Sng 3-H113

$4525093-\mathrm{H}_{1} \mathrm{t}_{3}$

$4235093-\mathrm{H}_{113}$

44 SOR3=HI13

3月9 50 B०स 113

3 S $5 \cap 83-H_{1} 13$

$3905093-\mathrm{H}_{113}$

$384500^{-H_{1}} 3$

$3915 \cap A 3=113$

3 月6 SOR3-H113

$39250 \cap 3=\mathrm{H}_{1} 13$

3ดค 50 ด्स 113
CHEN CLFAN aLCOA

MILE FINISH ALCOA

CHEN CLFAH aLCOA

CHEM CLFAN abCoA

MTLL TINISH AI,COA

CHEM CLFA aLCOA

mitic finish alicoa
MJLI RINISH ALCOA
$150 / 3$

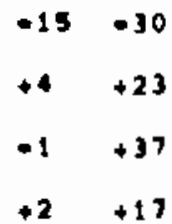

0,31

$+5+32$

$x-\operatorname{sect}$

$X-\operatorname{sect}$

$x=\operatorname{sect}$

$x-\operatorname{sect}$

$x$-SECT

$x-8 E C T$

$x$ - sect

$x=\sec T$
LIT 253

L[T 263

LIT 26J

LIT 2G 3

[IT 26]

LIT 26J

LIT 263

LIt 26]

LIt 263

LIt 24]

LIT 263

LIT 263

LIT 243

LIT 263

BARMAC ALCOA IT

ALCOA 40

GARMAC ALCOA I7

ALCOA 49

BARNAC ALCOA 47

ALCOA 66

BARMAC ALCOA 67

ALCOA 61 
TAPLE II

MISCELTLANEOUS CRRRCSIUN TNFORMATIUN

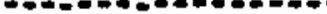
CQFVTCF STRESS PROPERTY METHOD ARTITTONAL SAMPIE DFSCHTOTTIN ATTACK STRESS PROPERTY METHOD

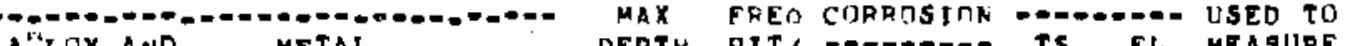

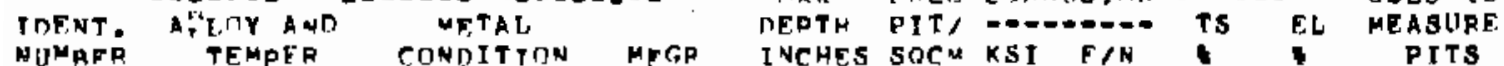
SEA HATEA EXPOSURE CUNDITIUN
FLOW FI/ TEMP 02 SAL CO2 COJ MARINE INCHES SOCM KSI PUN

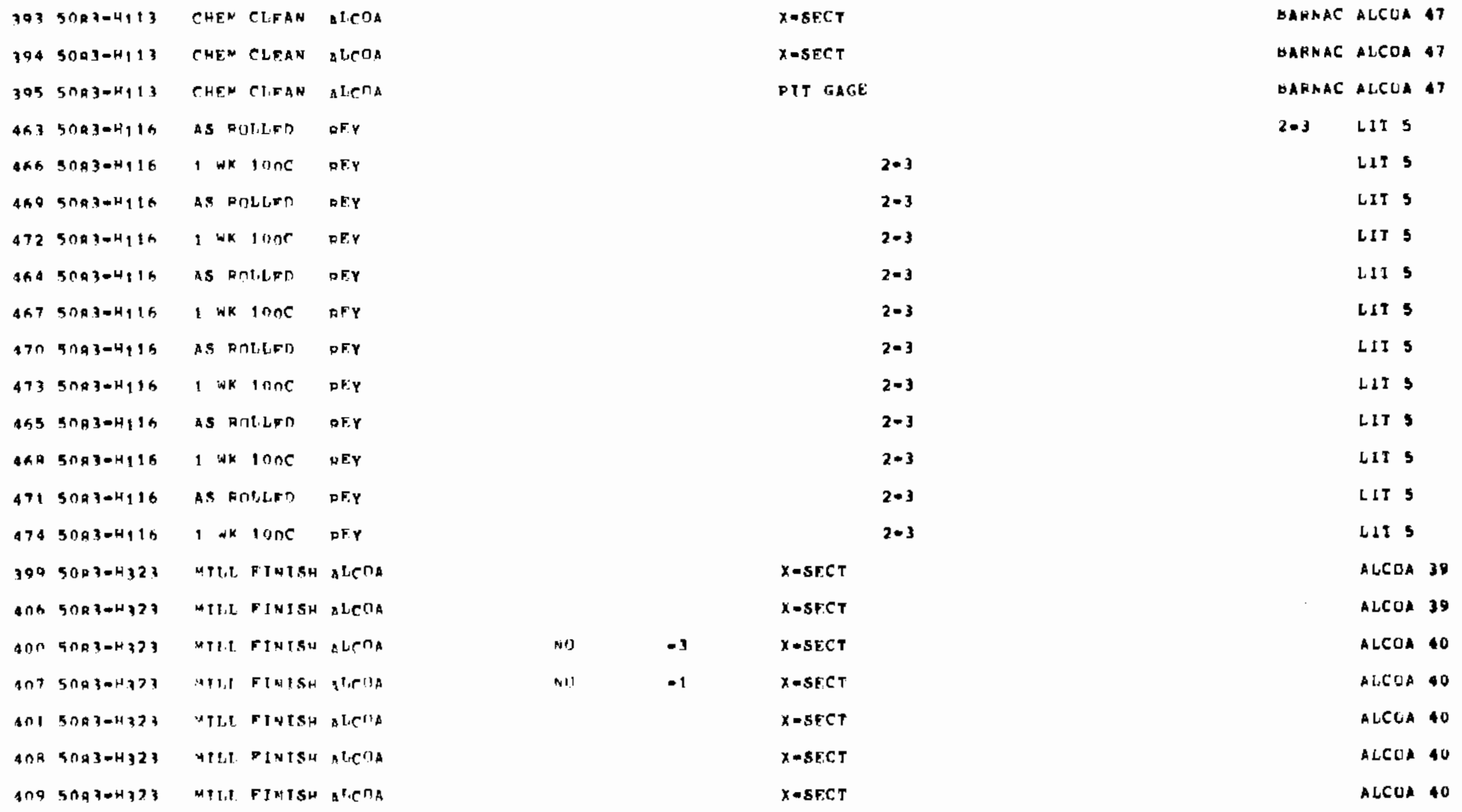


TABLE II

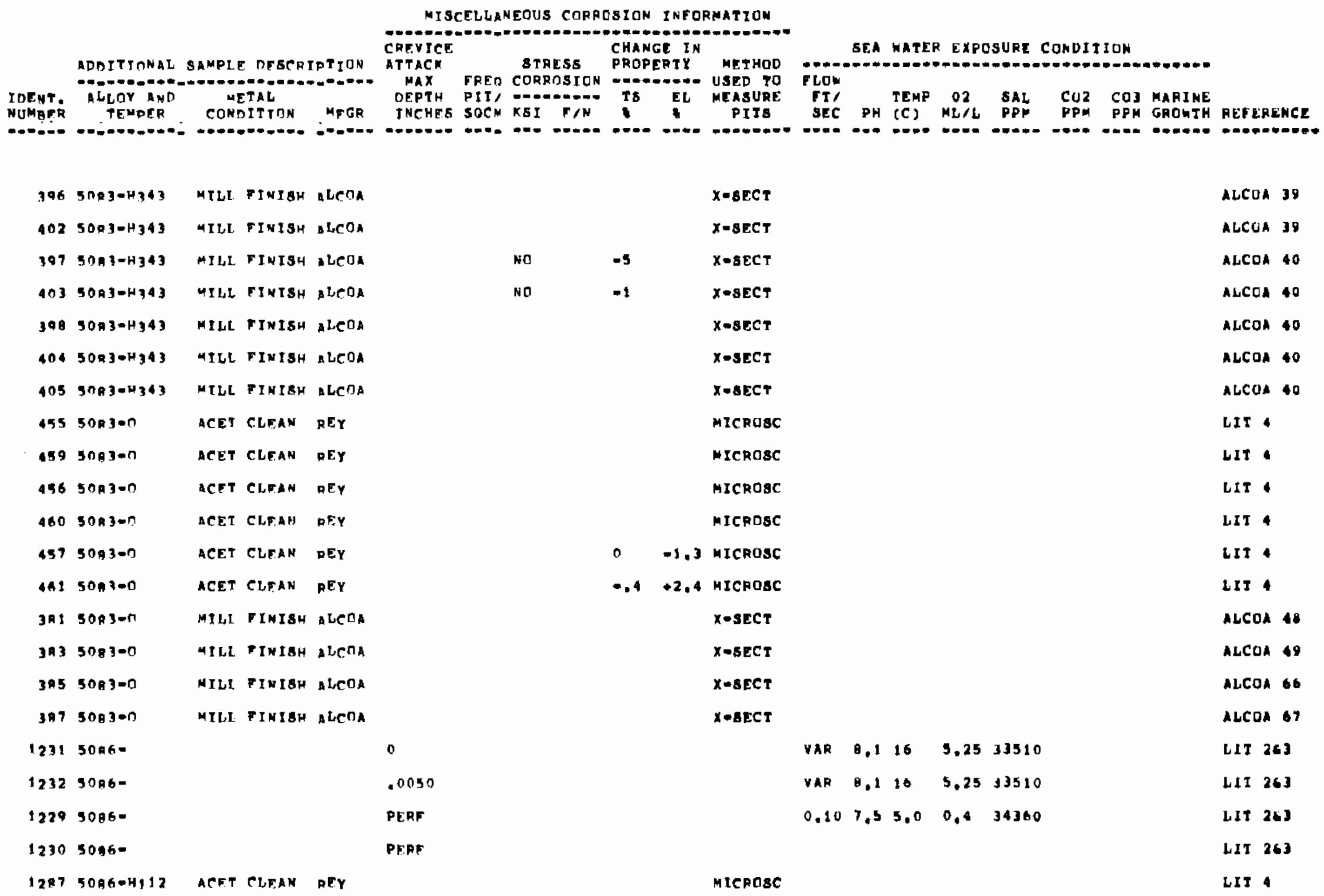


TAPIF II

*iscellaneois cophesion infGrmation

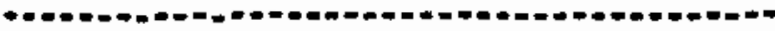

COFVICE

COEVICE THANGF IN

ADITTINAT. SAMPIF DESCRTOTION ATTACY HRE CURESB PROPERTY METHDD

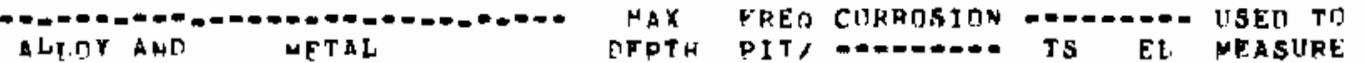

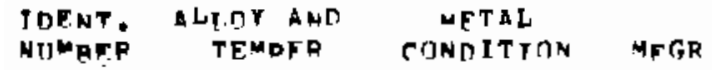
IFPTH PIT, MEHS SUCM KSI FIN IS EL MEASURE

SEA WATEP EXPCSURE CONDTTION BLDU FLOH $\begin{array}{llllll} & \text { FI' } & \text { TEMP } 02 & \text { SAL } & \text { COZ COS MARINE } \\ \text { TS SEC PH (C) MLIL PPM PPM PPM GPOHTH }\end{array}$

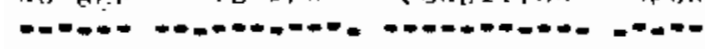

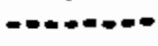
SEC PH (C) MLLL PPM PPM PPM GPOHTH REFLREMCE

\begin{tabular}{|c|c|c|c|c|}
\hline 1200 & $5 O A A_{-H} 12$ & $A C F T$ & CLFAN & \\
\hline 1298 & 50 A $6-\mathrm{H}: 12$ & ACET & CLFAN & \\
\hline 1201 & $5 \cap_{Q} 6-H_{1}+2$ & ACE.T & CIIAN & \\
\hline 1 วคด & $5006=\mathrm{H}_{1} 12$ & ACET & CLEAN & \\
\hline 1292 & R6. & ACET & CLFAA & \\
\hline
\end{tabular}

$12645096-4112$

$1255 \sin a=H_{1} 12$

12 an SOAS=H112

1246 SOR6-H,12

$12675 \cap R G=H_{1} 12$

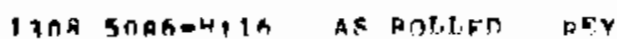

131 I 5OAfarith

13 ON SRRALPIA

$1323509 K=H 116$

1309 5ORA-H116

1312 SกคG-HIIA

1321 5ngh-HI16

1394 5nQR-HI15

1310 snes-4t1h

1313 SBRKAH, IS

1372 SORA-H116

1325 SחRK=H, 16

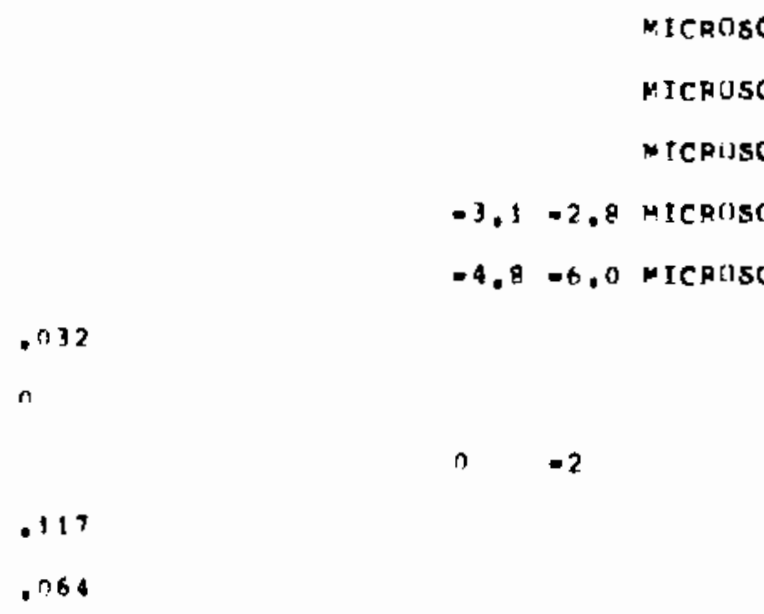

$$
\begin{array}{lllll}
0.05 & 7.4 & 2.3 & 1.6 & 34600 \\
\text { VAR } & 0.1 & 16 & 5.25 & 33510 \\
\text { VAR } & 9.1 & 16 & 5.25 & 33510 \\
& 4.5 & 5.18 & \\
4.5 & 5.18
\end{array}
$$$$
2-3
$$$$
2=3
$$$$
2=3
$$$$
2-3
$$$$
2 \cdot 3
$$$$
2=3
$$$$
2=3
$$$$
2-3
$$$$
2=3
$$$$
2=3
$$$$
2-3
$$$$
2-3
$$

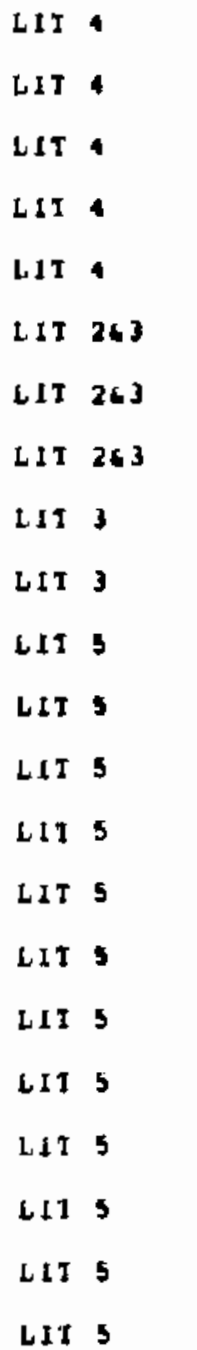


TAHLE IT

HISCELLANEOUS CORROSION INFORMATION

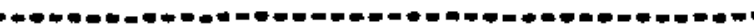

CREVICE

CREVICE STAESE IN

ADOITIMNAL SAMPLE DFSCRTPTTON

ATTACK BTAESE PROPERTY METHDD

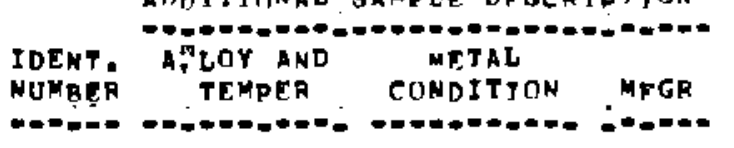

MAX TREO CORROSION -OOUOQOD UBED TO

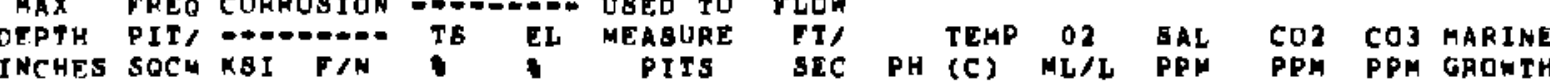
PITS SIC PH (C) ML/L PPM PPM PPM GAONTH REFERKMCE

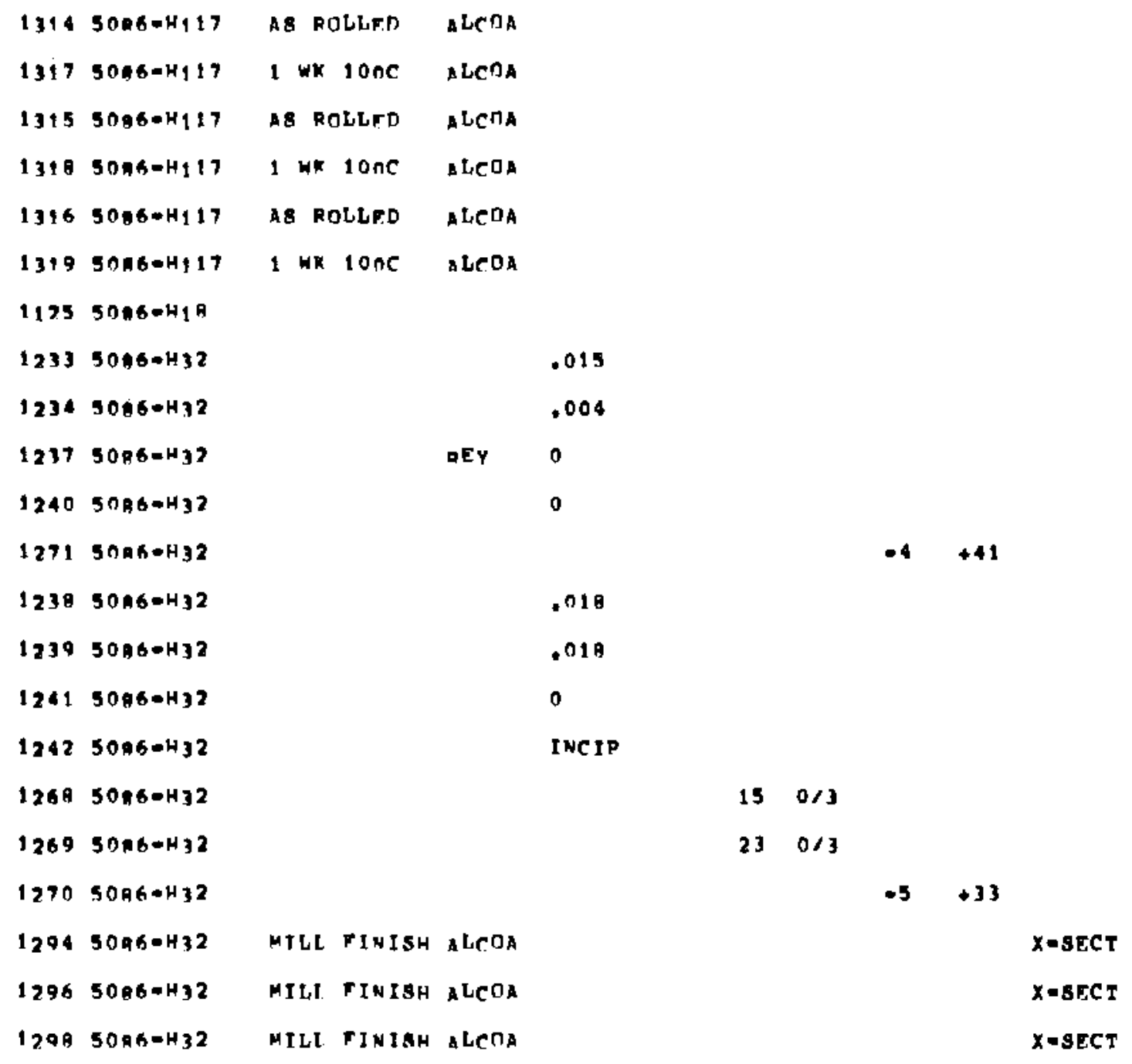

$2 \cdot 3$

$\mathbf{2}=\mathbf{3}$

$2 * 3$

$2-3$

$2 \cdot 3$

$2 \cdot 3$

$0.057 .42 .3 \quad 1.6 \quad 34600$

$0.107 .55 .0 \quad 0.4 \quad 34360$

VAR $0,116 \quad 5,2533510$

VAR $0,116 \quad 5,25 \quad 33510$

$0.107 .55 .0 \quad 0.434360$

VAR $0,116 \quad 5,2533510$

VAR $0,116 \quad 5,2533510$

$0.107,55.0 \quad 0.4 \quad 34360$

$0.107 .55 .0 \quad 0.4 \quad 34360$

$0.107 .5 \quad 5.0 \quad 0.4 \quad 34360$

$X$-SECT
LI 5

LIT 5

LIT 5

LIT 5

LIT 5

LIT 5

LIT 6

LIT 263

LIT 263

L1T 3

LIT 263

LIT 263

LIT 2GJ

LIT 263

LIT 263

LIT 263

LIT 26J

LIT 243

417263

ALCOA 40

ALCOA 49

ALCOA 60 
TAHL IT

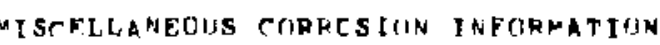
CDFVICF. CHANGE IN

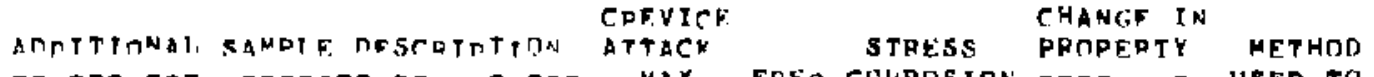
ATIDY AND INCHAS SOCM KSI F/N PITS

SEA WATER EXPOSUKE CONOITIUN
FLOW
FI' TEMP O2 SAL COZ CO3 MARINE
SEC PH (C) MLIL PPM PPM PPM GROMTH HESERENCE

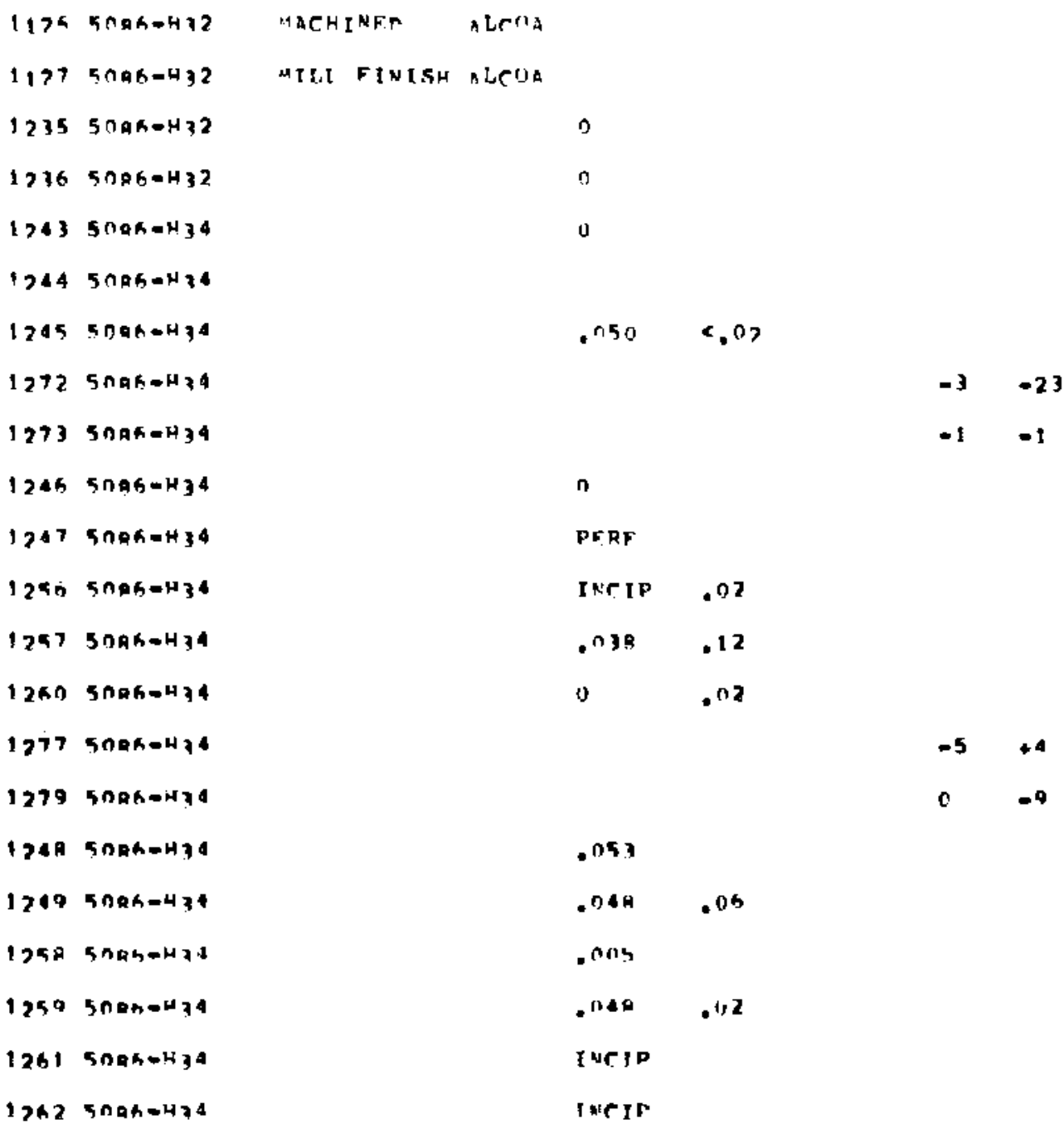

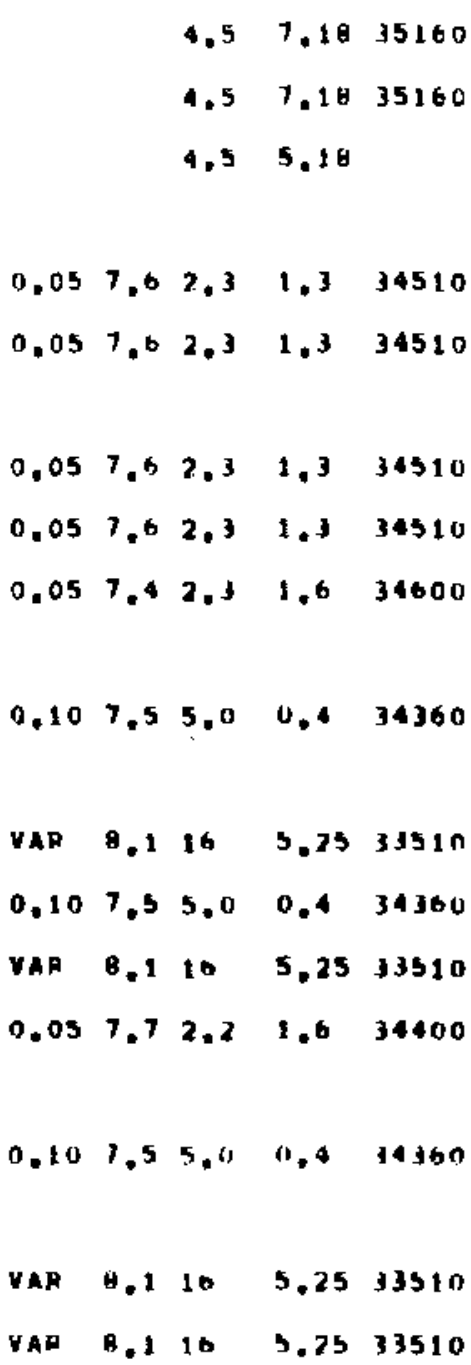


TAGLE II

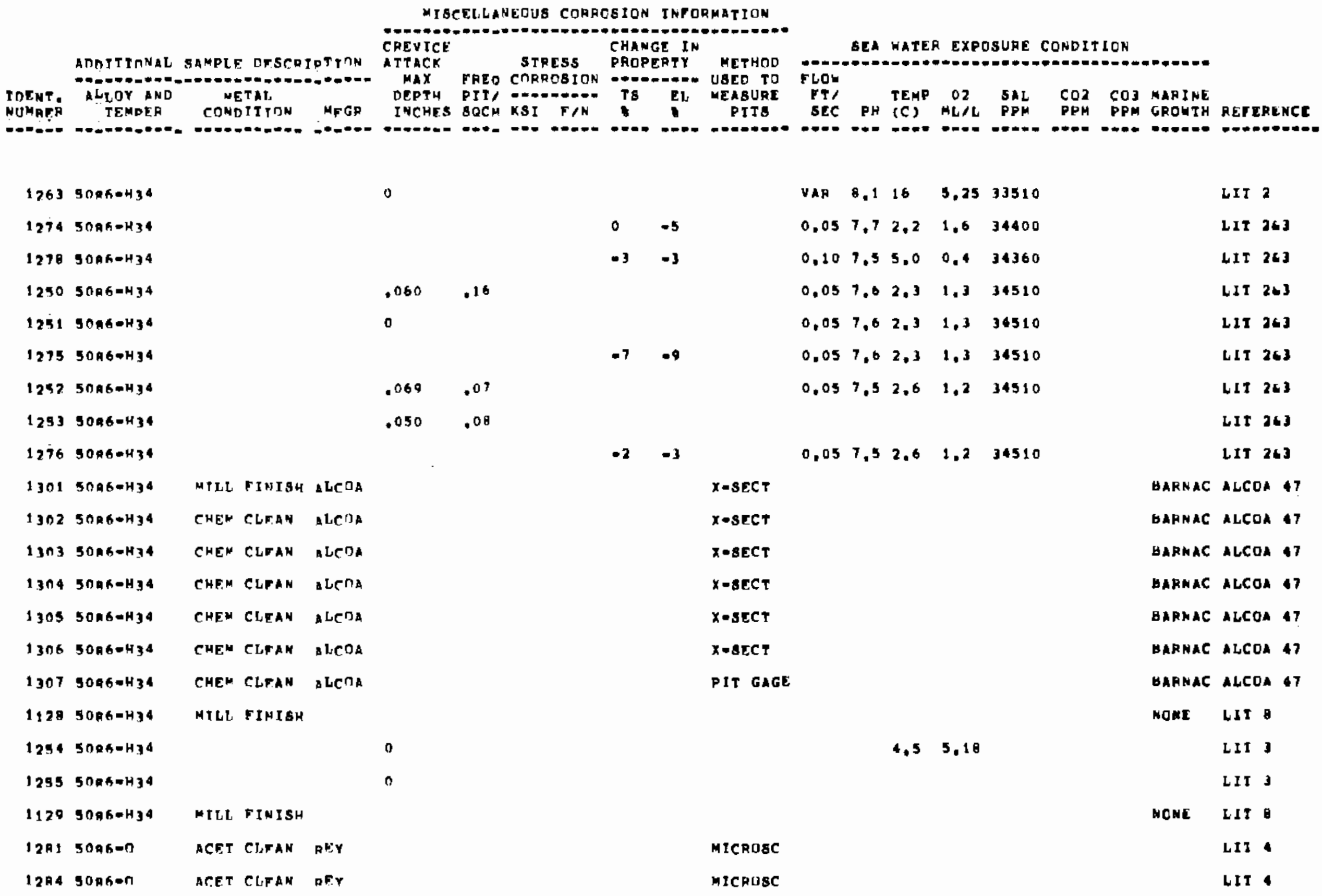


TAFI. II

MTSCETLAVEOIS CORFESION JMFORAATION Cupvich CHFVTCF CHANEF IN attack AMTITTMMAL SAMDLF DFSTDTETTCN TTACK STRFSS PROPFRTY METHOU - artid

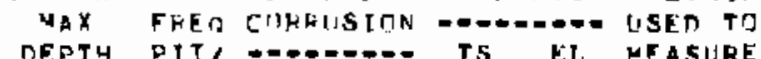
DEPTH PIT'

SFA WATEK EXPCSURE CONUITIUTH

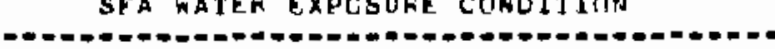
FLC:

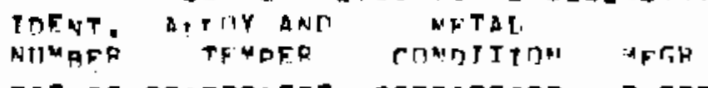

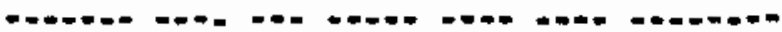
- PH (C) MLIL PPM PPH PPM GPLNTH REFERENCE

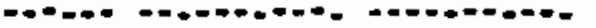

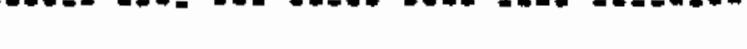

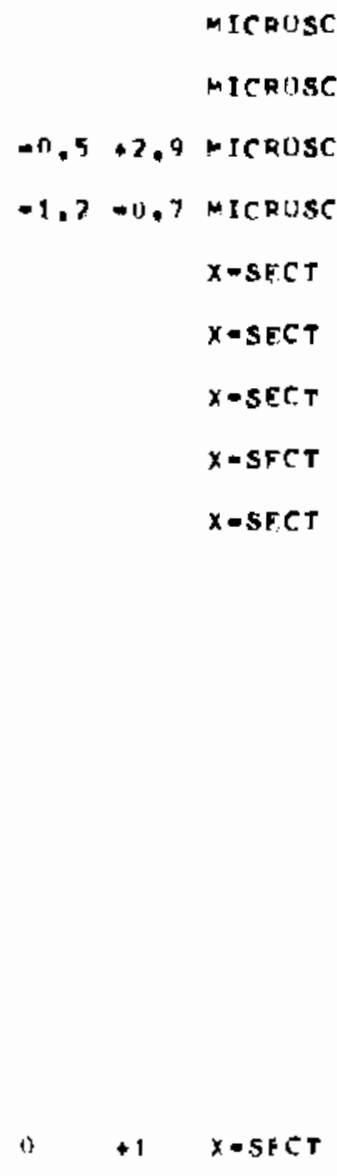


TARLE II

MTSCELLANEOUS CDRPCEION INTERMATION

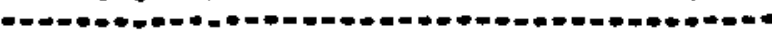

CRTICE

$\begin{array}{ll}\text { CRTVICE } & \text { CHANGE IH } \\ \text { MTTACK } & \text { STRESS PROPERTY METHOD }\end{array}$

AODTTIOMAL SAMPLE DFSCRTDTION

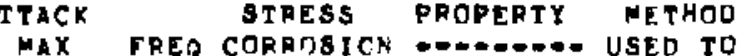

BEA WATER EXPOSUHE CONDITION
FLOW

IOENT, ALLOY AND METAL

MAX FRED CORAISICN H...... USED T FLOW DEPTH PIT, -...-. TS EL MEASUAE FT/ TEMP 02 SAL COZ CO3 HARINE INCHES SOCW KSI F/N SEC PH (C) MLL PPM PPM PPM GROHTH REFERENCE NUMBER TEMPER CONDITIDN DFGR

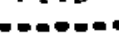

\begin{tabular}{|c|c|c|c|c|}
\hline 1063 & $5154-4.34$ & & & $A \operatorname{LCDA}$ \\
\hline 1064 & $5154-\mathrm{H}_{3} 4$ & & & $A L C O A$ \\
\hline 1055 & $5144-\mathrm{H}_{3} 4$ & & & $\triangle \operatorname{LCOA}$ \\
\hline 1066 & $5154-434$ & & & ALCOA \\
\hline 1075 & $5154-\mathrm{H}_{34}$ & MTLL & FINISH & $\triangle \mathrm{LCOA}$ \\
\hline 1076 & $5154-H 34$ & MILL & FINISH & $\operatorname{AtcOA}$ \\
\hline 1077 & $5154 \div \mathrm{H}_{34}$ & MILL & FINISH & aLCOA \\
\hline 1078 & $5154=434$ & MILL & FINISH & acha \\
\hline 1079 & $5194-\mathrm{H}_{3} 4$ & MTLL & FINISH & $\triangle \mathrm{LCOA}$ \\
\hline 10 O & $\left.54 \$ 4+H_{3}\right\}$ & $M I L I$ & FINISH & $\operatorname{Atccta}$ \\
\hline 1091 & $5154=\mathrm{H}_{34}$ & MILI. & FINISH & A LCOA \\
\hline IOA2 & $5154-\mathrm{H}_{34}$ & CHEF & CLFAN & ALCOA \\
\hline 1083 & $5154-\mathrm{H}_{34}$ & CHEN & CLFAN & $\triangle \mathrm{LCOA}$ \\
\hline 1094 & $5154-434$ & CHEM & CLFAN & $\operatorname{sic} n$ \\
\hline $10 R 5$ & $5154-\mathrm{H}_{34}$ & CHE & CLEAN & ALCOA \\
\hline 1096 & $59,94-\mathrm{H}_{3} 4$ & CHEM & CLEAN & ALCDA \\
\hline 10 ค? & $5154 * \mathrm{H}_{3} 4$ & CHEM & CLFAN & ALCOA \\
\hline 1028 & $5154=\mathrm{H}_{3} 9$ & ACET & CLFAN & DEY \\
\hline $\ln 91$ & $5154=13$ 月 & ACF.T & CLFAN & RF.Y \\
\hline toh9 & $5154-H_{3} 8$ & ACE T & CLEAN & REY \\
\hline 1092 & $5+94=43 R$ & ACE.T & CLFAN & DEY \\
\hline 1090 & $5154-H, 9$ & ACET & Cl.FAN & PE.Y \\
\hline
\end{tabular}

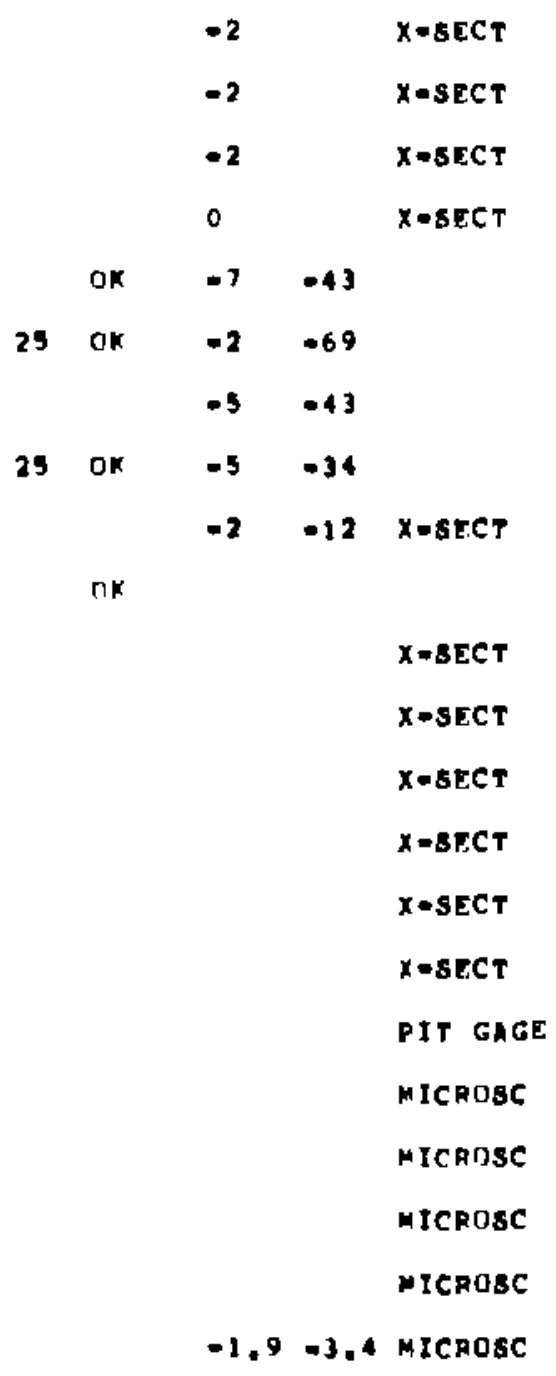

ALCOA 12

ALCOA 12

ALCOA 12

ALCOA 12

ALCOA 9

ALCOA

ALCOA 0

ALCOA

ALCOA

ALCOA

BARMAC ALCOA AT BARNAC alCOA IT

BARNAC ALCOA 47

BARMAC ALCOA 47

BARNAC ALCOA 47

GARMAC ALCOA 17

BARNAC ALCOA 47

LIt 4

LIT

LIT 4

LIt 4

LIT 4 
TAELE II

MISCFLLANEOIS CTIPRCSION TNFORMATION ADTTYIONAL. SARPIE DFSCRTTTITN CREVICF $\begin{array}{lll}\text { CREVICF } & \text { CHANGE IN } \\ \text { ATTACK } & \text { STRESS PROPFRTY METHOD }\end{array}$ ATTACK STPESS PROPFRTY METHON

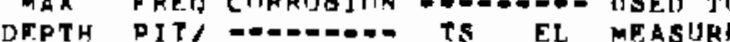
INCHES SOCM KSI F/N

\section{SEA hater EXPOSUNE CONDITIGN FLOW \\ FT/ TEMP O2 SAL CO2 CU3 MARINE} SEC PH (C) Mi/L PP PPM PPM GRONTH REFERENCE

(1)

\begin{tabular}{|c|c|c|c|c|}
\hline 1003 & $5154-H \backslash B$ & ACET & CLFAN & $D E Y$ \\
\hline 1071 & $5194=[3$ & MIUT & FIN IS H & $\Delta \operatorname{LrCl}$ \\
\hline 1072 & $51,4=n$ & MILI. & FINISH & $\Delta \mathrm{T}_{\mathrm{s}}$ \\
\hline 1073 & $5154=0$ & ${ }^{* 4}$ I LI & F I I I S H & $\Delta L C$ \\
\hline 1074 & $5154=0$ & MTLI & FIN【SH & $A$ \\
\hline 1092 & $5254-434$ & $M T L L$ & FINISH & \\
\hline 1053 & $5254=\mathrm{H} 34$ & $M A C^{P}$ & INED & Dl, C \\
\hline 1046 & $5254-\mathrm{H}_{3} 4$ & MTL,L. & FINISH & A \\
\hline $\ln 47$ & $5254-4,34$ & YTLL & FI시 I SH & $A I_{1}$ \\
\hline 1049 & $52,4-434$ & MACH & INET & $\mathrm{L}$ \\
\hline $105 n$ & $5264-H_{3}^{4}$ & MACH & INEN & \\
\hline $\ln 48$ & $52<4-134$ & $M T I, L$ & FIMISH & suc \\
\hline 1051 & $5254-1+34$ & $\operatorname{MACH}$ & INEN & Alien \\
\hline 1044 & $5346=$ & MILT & FINISH & \\
\hline 1045 & 51960 & MIT $\mathbf{Y}_{i}$ & FIN ISH & \\
\hline 1343 & 54440 & & & \\
\hline 1344 & 54540 & & & \\
\hline 1341 & 54540 & & & \\
\hline 1342 & $5444=$ & & & \\
\hline 1333 & $5454-432$ & ACF:T & CLFAN & $\not F Y$ \\
\hline 1337 & $5454=H 32$ & ACET & CI,F.AN & $D F Y$ \\
\hline 1334 & $5124-4>2$ & $A C F T$ & $\mathrm{Cl}_{2} \mathrm{~F} \otimes \mathrm{N}$ & D.Y \\
\hline
\end{tabular}

$-6.2=10 \quad$ ICROSC

$-10 \quad-60$

$-7 \quad-45$

$-3 \quad-19 \quad x-5 F C T$

nx.

30011

$30 \quad 0 / 1$

$x-\operatorname{srct}$

$x \rightarrow$ SEC T

$25 \quad 0 / 1$

$250 / 0$

$x \rightarrow \operatorname{SECT}$

$250 / 0$

$x=\operatorname{sect}$

$X=S F C T$

$\begin{array}{lllll}\text { VAR } & 0.1 & 16 & 5.25 & 33510 \\ \text { VAF } 9.1 & 16 & 5.23 & 33510 \\ 0.10 & 7.5 & 5.0 & 0.4 & 34360\end{array}$

MICAISTC

M J CRnsC

MICROSC
II 4

ALCOA

ALCOA $B$

ALCOA 8

ALCOA 8

ALCOA 62

ALCOA 62

ALCOA 17

BARNAC ALCOA 17

ALCOA 17

maRnac aLCOA 17

NONE ALCLA 17

NONE ALCOA IT

ALCOA 25

ALCOA 25

LIT 263

LIT 263

LII 263

iI I 243

MAHNAC LIT 4

BARNAC III

HAHNAC LIT 4 
TAELE I I

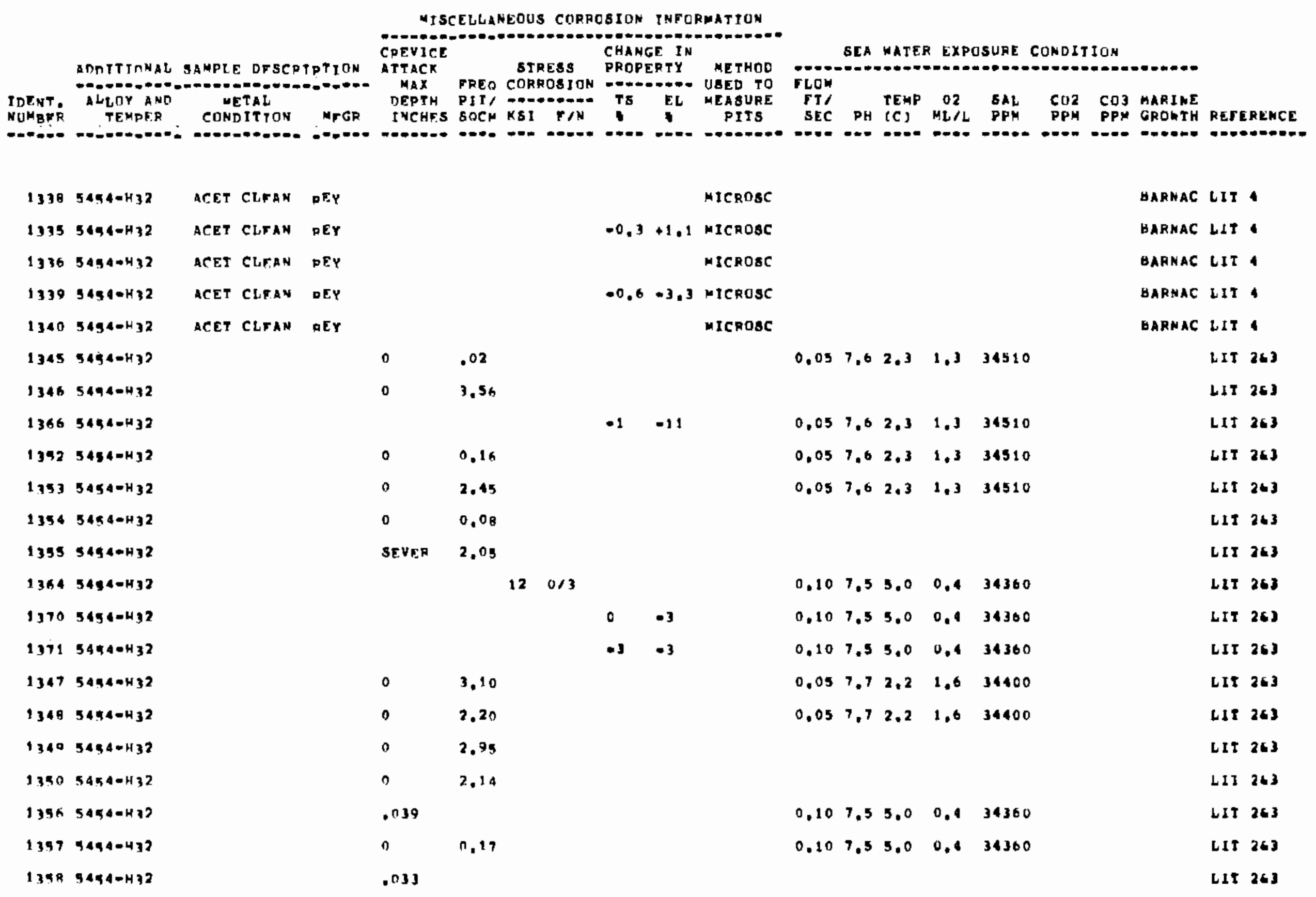


TAELK II

MISCEI,LANEOUS CORFCSION TNFTRMATION

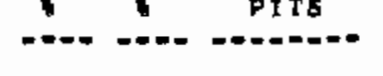

0.05

$13425454-\mathrm{H}_{32}$

$13635454 \div 432$

$13655454=132$

$13 \times 75454-432$

$1368 \quad 5454=432$

$13725454-432$

$13735454-437$

$13515454-432$

$13695454=432$

$18355454 \times H_{32}$

$123654 \times 4=472$

1838 $5454=\mathrm{H} 32$

$18295454-432$

1月41 5454-H32

$19425454=\mathrm{H32}$

$19445424=432$

$10155454-\mathrm{H}_{3} 3$

$137454 \times 4-\mathrm{H} 34$

i.21 $413,4-424$
$120 / 3$

$120 / 3$

$\begin{array}{ll}-2 & -22 \\ -2 & -7 \\ -4 & -27 \\ -4 & -6\end{array}$

$0 \quad 0.93$

MILI INISH ALCOA

NILE FINISH DICOA

MILI. FINISH alcó

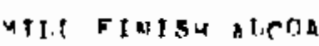

ATLE FIISU BITHA

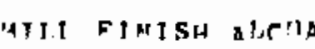

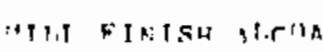

"TI.J FIMSH ATCOA

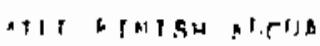

Chen ChaA alom
$-16 \quad-34$

$-16 \quad-34$

SEA WATEK EXPQSURE CONHITION

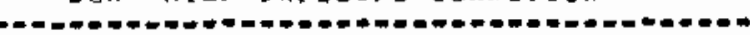

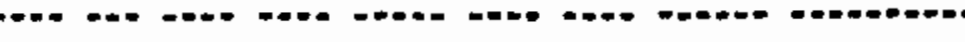

\section{$x \rightarrow \operatorname{SEC} T$ \\ $X=\operatorname{SECT}$ \\ $X-\operatorname{SECT}$ \\ $x-\operatorname{SEC} T$ \\ $x=5 F C T$ \\ $x=E \mathrm{ECT}$ \\ $x+5 \sec$ \\ $x=\operatorname{sect}$ \\ $x=S F, C T$ \\ $X-\operatorname{SECT}$}

LIT 2 G

LIT 263

LII 243

LIT 263

LII 263

L I I I 25$]$

LII 243

LIT 243

LIT 263

LIT 24J

LII 263

LIT 263

ALCOA 40

ALCOA 48

ALCOA 49

ALCDA 49

a LCOA bo

a LCOA bo

AISCUA 67

ALCOA 67

HAFNAC ALCUA 47

HARNAC ALCUA 47 
TAELE II

MISCELLANEOUS CORPOSION INFGRMATION

CREVICE

$\begin{array}{lll}\text { CFFVICE } & \text { CHANGE IN } \\ \text { ATTACK } & \text { STRESS PROPERTY METHOD }\end{array}$

ADDITIONAL SAMPIE DESCRIPTION

USEDTO

DEPTH PIT, CRASION TS EL MEABUAE

INCHFS SOCM KSI FAN

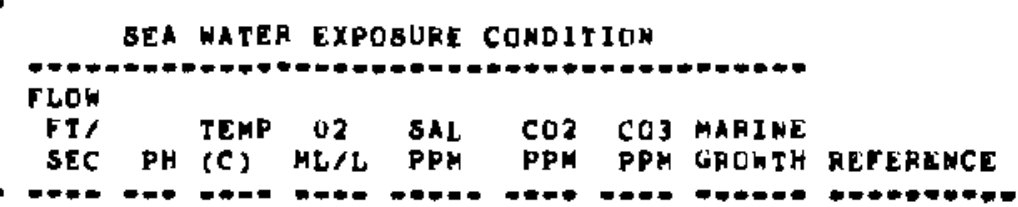

\begin{tabular}{|c|c|c|c|c|}
\hline 328 & 549 & CHEM & CLFAN & BLC \\
\hline 329 & $5454-H_{3}^{4}$ & СHEN & CLF,AN & ALCnA \\
\hline 330 & $5454-434$ & CHEM & CLEAN & LCOA \\
\hline 331 & $5454-1134$ & CHEN & CLEAN & ALCDA \\
\hline 332 & $5454-1344$ & CHEN & CLFAN & ALC \\
\hline 042 & $54,54-\mathrm{H}_{3} 4$ & MACHI & INEn & ater \\
\hline 1043 & $5454=H 34$ & MILI & FINISH & AlCOA \\
\hline 1834 & $5454-n$ & $4 \pi L L$ & FINISH & ALCC \\
\hline 1829 & $5454=n$ & MILI & FINI $8 \mathrm{H}$ & ALCOA \\
\hline 1830 & $5454=0$ & YTLt & FINISH & ALCOA \\
\hline 1931 & $5494 * n$ & NItL & FINISH & a Lec \\
\hline 1832 & & MILL & FIMISH & \\
\hline 1833 & $5454=n$ & MILI & FINISH & sLenA \\
\hline 1837 & $5444=0$ & $4 T L I$ & FINISH & $\triangle \mathrm{LCt}$ \\
\hline 1840 & $5454=0$ & MILI & FINI BH & $\operatorname{LCOA}$ \\
\hline 1843 & $54.9=17$ & WfLt & FINISH & $\triangle L C O A$ \\
\hline t a 24 & $5454-\pi$ & MILL & FINISH & $\operatorname{Atcas}$ \\
\hline 1 B 25 & $5494-5$ & MILL & FINI SH & ALCOA \\
\hline 1826 & $5454-\pi$ & $H I[, L$ & FINISH & ALCOA \\
\hline 1827 & $5454-n$ & MTLI & FIN I BH & $\Delta \operatorname{LCOA}$ \\
\hline 1829 & $5454=0$ & $M\left[I_{2} I\right.$ & FINI 84 & $A \mathrm{LCOA}$ \\
\hline 956 & $545 x-H 1$ & As $F X$ & XTAUUED & $\$ 1$ \\
\hline
\end{tabular}

$X-5 E C T$

X-8ECT

$\mathrm{X}=\mathrm{sec} T$

$\mathrm{X}-\mathrm{BECT}$

PIT GAGE

$4.8 \quad 5.18 \quad 35160$

$4.8 \quad 5,18 \quad 35160$

$x=\sec t$

$x=\operatorname{SEC} t$

$x-\operatorname{sect}$

$x-s E C t$

$x-\sec r$

$x-\operatorname{sect}$

X-SECt

$x-s \in c t$

$x-\operatorname{sect}$

$X=\mathbf{E C T}$

$\mathrm{X}-\mathrm{seCT}$

$x=8 E C T$

$x=\operatorname{sect}$

$x \cdot \sec t$

$x=\operatorname{sect}$
GARNAC ALCDA 47

BARMAC ALCOA 47

BARHAC ALCOA 47

GARNAC ALCUA 47

BARNAC ALCOA 47

NOHE ALCOA 7

NONE ALCOA?

ALCOA 40

BARAC ALCOA 36

HAFNAC ALCOA 30

DARMAC ALCOA 36

BARNAC ALCOA 36

BARNAC ALCOA 30

ALCOA 49

ALCOA 60

ALCOA 67

GAHNAC ALCOA 37

BARNAC ALCOA 37

GAHNAC ALCOA 37

BARNAC ALCOA 37

BARMAC ALCOA 37

bahHAC ALCOA 42 
TABC I I

MISCFILANECLIS CORRCSION IMFOAMATIUN

CBevicF

CPEVICE

\section{STRESS CHANGE IN}

PROPERTY

WETHOD

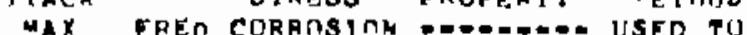

AMRTTTONAL SANPIE RESCRTOTJON

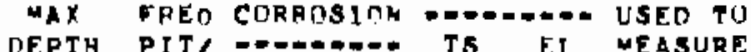
INCHFS SOCM KSI F/N TS PITS UFTAL
CONDITTCN MFGR

1 PITS SEC

SEA WATER EXPUSUAE CUNUITIUN

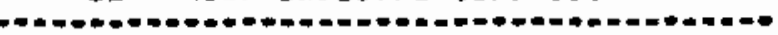

FT/ TEMP 02 SAL COZ CO3 MAHINE

SEC PH (C) ML/L PPM PPM PPM GROATH REFEHLNCE

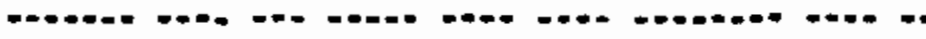

\begin{tabular}{|c|c|c|c|}
\hline 959 & $5455=H+12$ & AS $=X T R 1 Y D F T$ & alcoA \\
\hline 960 & $5456-H, 12$ & AS FXTRUNFn & $\triangle \mathrm{LC} \cap \mathrm{A}$ \\
\hline 957 & $5456-H_{1} 12$ & As ExTR11DFT & $A C^{\prime r J A}$ \\
\hline $9 \times 1$ & $54 \& A=H, 12$ & AS FXTKIDE.T & $A L C O A$ \\
\hline TR2 & $5446=\mu g 1 ?$ & AE FXTRINEn & ${ }_{A} \operatorname{LCOA}$ \\
\hline 958 & $5456-4112$ & AS FXTPUDF.T & $A L C O A$ \\
\hline 963 & $545 K=H_{1}+2$ & AS FXTRIMFn & $\triangle L C O A$ \\
\hline $9 \times 4$ & $5496-H_{1} 12$ & AS FXTR(IIST & BI.COA \\
\hline 065 & $5456=4,16$ & AE POLLFn & PEY \\
\hline 979 & $5456=-4116$ & AS ROLIFO & DF.Y \\
\hline 979 & $545 K=H \& 1 A$ & $t$ wa $\operatorname{lon} c$ & DFY \\
\hline $9 \times 6$ & $54 k h=H_{1} 116$ & As F(YLIFD) & DFY \\
\hline 975 & 5446-4 116 & AR PחLLEN & $O E Y$ \\
\hline $97 R$ & $5456-H_{11} \mathrm{~h}$ & 1 Wh $1 n n r^{\circ}$ & EFY \\
\hline $9 \times 7$ & C4AS-H, 1A & As ROLl,Fn & P*:Y \\
\hline 976 & $545 K=H_{1} 1 \mathrm{~h}$ & As ant,linn & PEY \\
\hline 974 & $545 \mathrm{k}-1+11 \mathrm{~m}$ & t ak lone & DF.Y \\
\hline 969 & 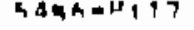 & A.s $R \cap[.1 .5 n$ & Iirfin \\
\hline 071 & $414 n+14117$ & $1+k \operatorname{lon} r$ & AICIIA \\
\hline man & $5156-4117$ & 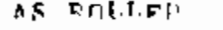 & ATRA \\
\hline 947 & $545 A=4117$ & $7 \mathrm{kr}$ ting & $\Delta \operatorname{tin} \Delta$ \\
\hline $7 \mathfrak{k}$ & 5 & $14,15+1$ & n \\
\hline
\end{tabular}

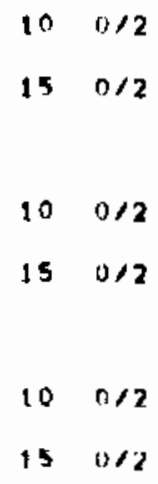

15012
BARMAC ALCOA 12

GAHMAC ALCOA 42

BAPAAC ALCUA 43

WAHAAC ALCOA IJ

BARNAC ALCUI IJ

BAPMAC ALCOA 45

GARNAC ALCOA 45

GARNAC ALCOA 45
L11 5
LII 5
LII S
LII 5
LIT 5
LII 5
LII 5
LII 5
LIT 5
LII 5
LIT 5
LIT S
LIT 5
UII 5 
TAPLE IT

MISCELLANEOUS CORRESION INFORMATION

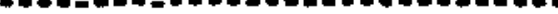
CPEVICT CHANGE IN

ATTACK STRESS PROPERTY METHOD

ADAITITNAL SAMPLE DFSCDIPTION

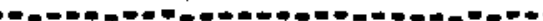

IDENT, ATLOY AND METAL
NUMAR TEMPA CONDITTON MFGR INCHES SOCN KSI F/N
NUMAER TEMPEF, CONOITTON MFGR

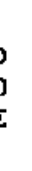

SEA WATER EXPOSURE COHDITION

FLOW
FT/ TEMP O2 SAL CUZ CQJ MARIME
SLC PH (C) ML/L PPM PPM PPM GROWTH REFEAKNCE

\begin{tabular}{|c|c|c|c|c|c|c|}
\hline 972 & $5496-4197$ & 1 WK $100 C$ & $\operatorname{LenA}$ & & & \\
\hline 9月1 & $5456-H 117$ & AS FOLLED & $\triangle \operatorname{LCOA}$ & & & \\
\hline 983 & $5446-4,17$ & 1 wh $10 n c$ & $A \operatorname{LCOA}$ & & & \\
\hline 970 & $5456-4117$ & AS FOLLED & $A C_{A}$ & & & \\
\hline 973 & $54560 \mathrm{H}_{117}$ & 1 Wh tonc & ALCOA & & & \\
\hline 1421 & $5456-H_{14}$ & MILL FINISH & $\triangle \operatorname{LCOA}$ & & & \\
\hline 1423 & $5456=H 14$ & MILL FINISH & alena & & & \\
\hline 930 & $5436-H_{2} 4$ & MILL FINISY & ALCOA & & & \\
\hline 933 & $5456 \cdot \mathrm{H}_{2} 4$ & CHEN CLEAN & acon & & & \\
\hline 934 & $5456 \cdot H>4$ & MILL FINISH & a LeOA & & & \\
\hline 931 & $5454-H_{2} 4$ & CHEM CLTAN & $A C^{\prime} O_{A}$ & & & \\
\hline 935 & $5456-1424$ & CHEN CLFAN & $A L C O A$ & & & \\
\hline 932 & $5456-\mathrm{H}_{24}$ & CHEM CLFAN & ALCOA & & & \\
\hline 936 & $5436-\mathrm{H}_{2} 4$ & CHEN CLTAN & $\mathrm{ALCOA}$ & & & \\
\hline 937 & $5456=\mathrm{H}_{2} 4$ & CHEM CLFAN & ALCOA & & & \\
\hline 954 & $5456-H 311$ & As AOLtFD & $\triangle \operatorname{LCDA}$ & & & \\
\hline 955 & $5456-H 311$ & 48 AnLLFD & ACOA & & & \\
\hline 1000 & $5436-\mathrm{H}_{3} 32$ & & & .0050 & & \\
\hline $\ln 10$ & $5456-1432$ & & & .021 & & \\
\hline $\ln 40$ & $5436-H 32$ & & & & 20 & $0 / 3$ \\
\hline $\ln 41$ & $5456-132$ & & & & 30 & $0 / 3$ \\
\hline
\end{tabular}

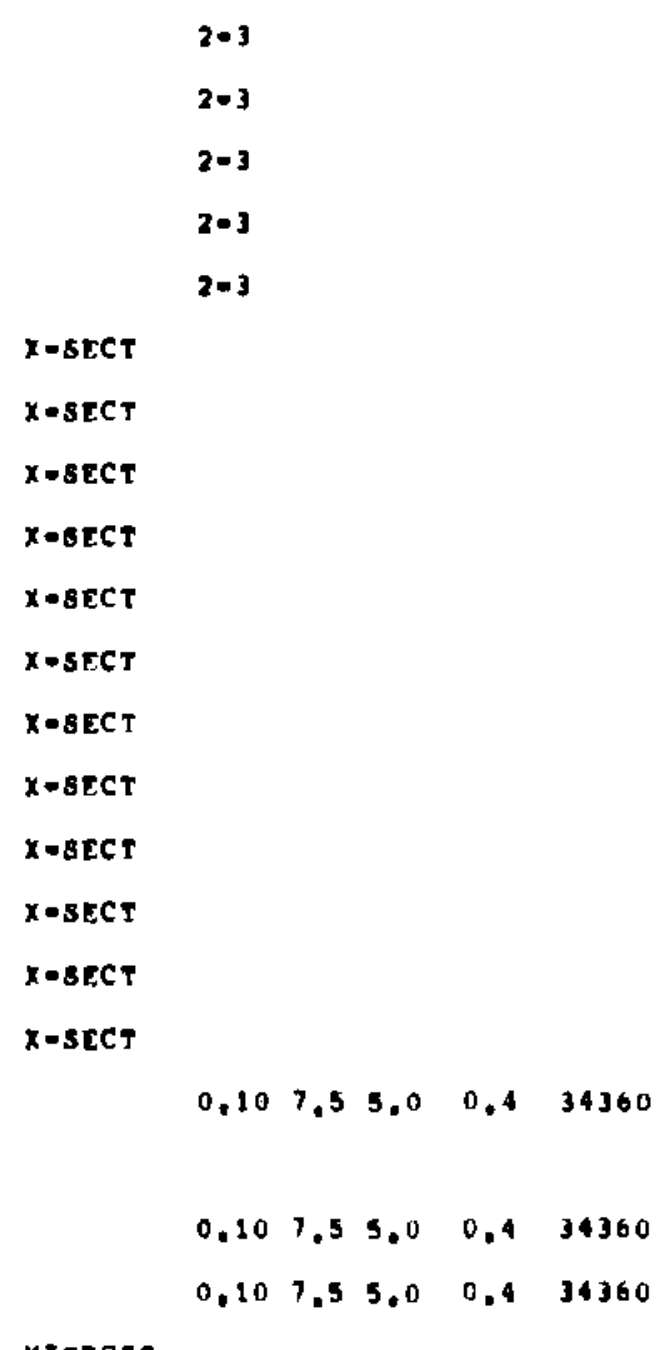

LIT 5

LIT 5

LII 5

LIT 5

LIT 5

ALCOA 29

ALCOی 29

HARMAC ALCOA JH

GARNAC ALCOA 32

ALCOA 33

GARMAC ALCOA 32

ALCOA 32

BARNAC ALCOA 32

ALCOA 32

aLCOA 32

ALCOA 52

ALCOA 52

LIT 243

LI 26 ]

(1) 243

LIT 243

BARMAC LIT 
TAHE II

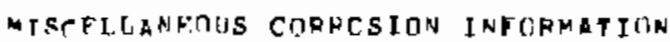

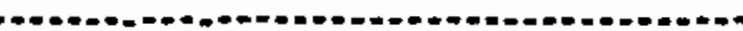

CRFVICF.

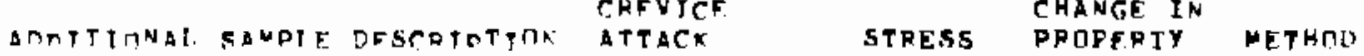

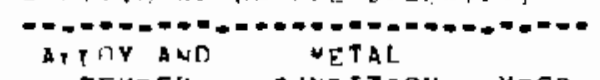

TDF.MT.

MTITTTINA

MAX FREO CIORROSIUN -....... USED TU

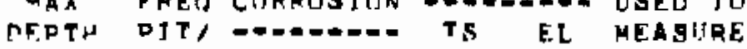

TNCHES SUCM KSI FIN 1 PITS

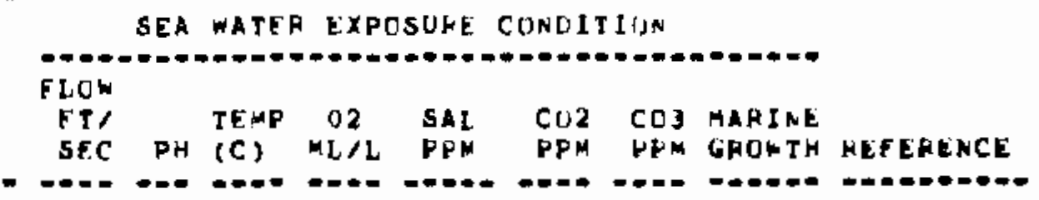

\begin{tabular}{|c|c|c|c|c|c|}
\hline 1005 & $\times 4 \times A=H \geq 21$ & $\operatorname{arst}$ & CT.FAN & $F^{F Y}$ & \\
\hline $\ln n 4$ & $545 K=4 \geq 21$ & ACF.T & CLFAN & DFY & \\
\hline 1007 & $54 \times 6=4721$ & $\operatorname{ArET}$ & $C\left[F_{A}{ }^{N}\right.$ & OFY & \\
\hline 1005 & $549 k-4>21$ & $A C F T$ & CLFAN & DFYY & \\
\hline $\ln n$ & $54260+321$ & $A C E T$ & CTEAN & OFY & \\
\hline 994 & $54 K K-U>>1$ & & & & \\
\hline 1011 & $54 \times 6-4 \geq 21$ & & & & StICHT \\
\hline $1 \cap 12$ & $5456-4321$ & & & & .11730 \\
\hline QRค & $5455-H 321$ & & & & \\
\hline 990 & $545 k-4 \geq 21$ & & & & \\
\hline 1019 & $54 \times x-4 \geq 21$ & & & & $.03 n$ \\
\hline 1020 & $5456-43 \geqslant 1$ & & & & . \\
\hline 1073 & $5455=4721$ & & & & 0 \\
\hline 995 & $5+5 k=4321$ & & & & \\
\hline QRQ & $5454-4321$ & & & & \\
\hline $1 \cap 13$ & $3456-4321$ & & & & $.175 n$ \\
\hline $\ln 4$ & $5456-4321$ & & & & .02 \\
\hline $\ln 71$ & $545 k-113>1$ & & & & .1144 \\
\hline $\ln ?$ & $545 x+4 \geq 21$ & & & & .1232 \\
\hline $\ln 24$ & $54 \times h=4321$ & & & & 0 \\
\hline $1 n>5$ & $545 k=4324$ & & & & . nna \\
\hline
\end{tabular}

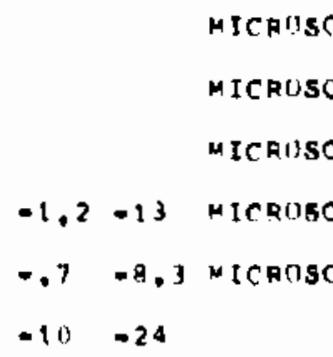

$0.057 .6 \quad 2.3 \quad 1.3 \quad 34510$

$0.057 .6 \quad 2.3 \quad 1.3 \quad 34510$

$0.107 .55 .0 \quad 0.434360$

VAR $0.115 \quad 5.2533310$

$0.107 .55 .0 \quad 0.4 \quad 34360$

VAR $\quad 8.1 \quad 15 \quad 5.2533510$

$0.057 .7 \quad 2.2 \quad 1.6 \quad 34400$

0.107 .55 .00 .434360

$0.05 \quad 7.72 .2 \quad 1.6 \quad 34400$

0.107 .55 .00 .434300

VAP A.1 IS $5.25 \quad 33510$

VAF $0.1155,2533510$

$0.057 .02 .3 \quad 1.3 \quad 34510$
DARNAC LIT

DARNAC LIT A

DARHAC LIT

BAHNAC LIT 4

DARNAC LIT

LII 263

LIT 2

LIT 263

LII $2+3$

LII 263

LIT 263

LIT 243

LIt 2 s 3

LII 243

LIT 243

LIT 243

[1] 243

LII 263

$L I I \quad 2+3$

L1I $2+3$

l1 203

LIT 243 
TAHE II

MISCELLANEOUS CORPOSTON TNFORMATION

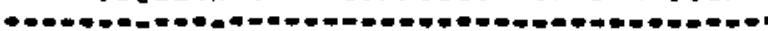
CREvice STREBS PHAREETY IN METHQO AMDITTRNAL SAMPLE DHSCOIDTION

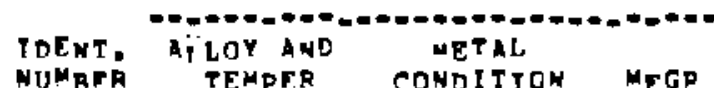
ATACK

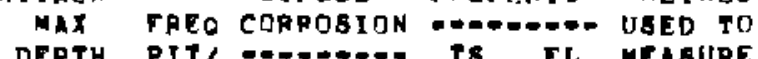

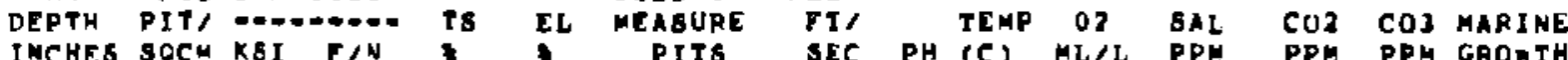
GEA WATER EXPOSURE CUNDITION
FLOW

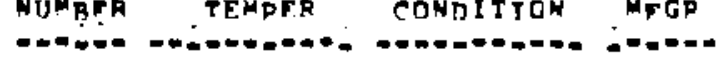
COE SOCM K8I FIN SEC PH (C) ML/L PPH PPM PPH GRONTH REFERENCE

\section{$10159456-H 321$}

$\ln 165456-4321$

$14195454-4321$

997 5456-4321

1017 S45h-H321

$10185456=4321$

$0235456-4321$

$13775454-4321$

1378 5446-H321

13月0 $5456-4321$

13月7 5456-H321

$13945456-4321$

$1401545 h=N 321$

0345456-13321

1391 5456-H321

$13 \mathrm{HB} 5496-\mathrm{H}_{321}$

$13055456-4321$

$14025454-4321$

$14475456=4321$

$14785456 * 4321$

$9255496-4321$

13 月2 5456-H321

\begin{tabular}{|c|c|c|}
\hline MACHI & NEn & $\triangle L C \cap A$ \\
\hline M I LL & rt4 IsH & ILETA \\
\hline $\mathrm{AACHI}$ & INEn & Acton \\
\hline MACHI & INEn & $A \operatorname{Lco} A$ \\
\hline MJLL & INISH & ILt \\
\hline MILI. & FIntsh & ALCDA \\
\hline YILL & FINI BH & $A L C O A$ \\
\hline $41 \mathrm{IL}$ & FINISH & ALC \\
\hline CHEF & CLFAN & $A L C D A$ \\
\hline CHEM & CLEAN & ALCOA \\
\hline CHEF & CLERN & stent \\
\hline CHEN & CLEAN & ALCDA \\
\hline CHEW & CLFAN & abean \\
\hline $\mathbf{M I} \mathbf{I}_{\mathrm{r}} \mathrm{t}$ & FINI 8 H & ALCDA \\
\hline M TLL & FINISH & ALCOA \\
\hline CHEN & CITEAN & \\
\hline CHEM & CLEAN & $\Delta \operatorname{LC} T A$ \\
\hline
\end{tabular}

$.025 \quad 0.02$

.0450 .09

$30 \quad 0 / 1$

$\begin{array}{llll}.037 & 0.11 & .4 & -6 \\ .046 & 0.23\end{array}$

$x-5 E C T$

$30 \quad 0 / 1$

$30 \quad 0 / 1$

$X-\sec T$

$x=S E C T$

$x \rightarrow \sec T$

X-8ECT

$x=\operatorname{sect}$

$\mathrm{X}-\mathrm{sect}$

$x$-sect

$x$-sect

X-8ECT

$x$-stet

THECT

$x$-sect

$x-\operatorname{sect}$
$0.057 .02 .3 \quad 1.3 \quad 34510$

LIT 2 t3

LIT 263

ALCOA 62

$0.051 .52 .6 \quad 1.2 \quad 34510$

LIT 263

$0.057 .52 .6 \quad 1.2 \quad 34510$

411263

L1T 263

GARNAC ALCOA 47

ALCOA 17

GARNAC ALCOA 17

Bapmac aLCOA 47

BAPMAC ALCOA 47

BARMAC ALCOA 47

BARMAC ALCOA 47

BARMAC ALCOA 47

GARMAC ALCOA 17

GARMAC ALCOA 47

GARNAC ALCOA 47

HARNAC ALCOA 47

alcua dy

ALCOA 40

BAHNAC ALCOA 47

BafNAC ALCOA 47 
TARLE II

MISCFLLANEOUS CORHCSITN INTCRMATION CRFVICE CHANGE IN CRFVTCE
ATTACK SHANGE IN
STRESS PROPERTY METHOD ADETTIONAL SAMPTE DFSCEIETTON

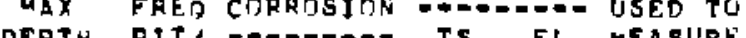
DEPTH PIT, FOAOHED TS EL FAS TEMP UZ SAL COZ COJMARIAE

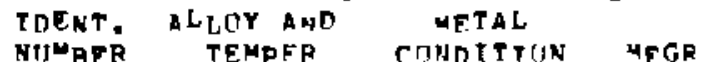
年 SEA NATER EXPOBUHE CUNDITIUN 年 SEC PH (C) MLAL PPM PPM PPM GROMTH KEFCRENCE

\begin{tabular}{|c|c|c|c|c|c|}
\hline 1309 & $5456-H 321$ & CHEM & CLFAN & $\triangle D_{C O A}$ & $x=\sec \tau$ \\
\hline 1396 & $5456=+1321$ & CHEM & CLEAN & $\triangle \mathrm{LCOA}$ & $x=\sec t$ \\
\hline 1403 & $5446-H_{3} 21$ & CHEN & CLFAN & $A L O A$ & $X-S E C T$ \\
\hline 1410 & $545 K-H 321$ & МтLI. & FINISH & $\triangle \operatorname{trCOA}$ & $X-S E C T$ \\
\hline $141:$ & $5456-H 321$ & MILI & FINISH & $\triangle L C O A$ & $x-5 E C T$ \\
\hline 926 & $5455-H 32 t$ & CHEN & CLIFAN & $A B C O A$ & $x=8 \mathrm{ECT}$ \\
\hline 13ค3 & $5456=4721$ & CHEM & CLIFAN & $B L C O A$ & $X=S E C T$ \\
\hline 1390 & $5456-+1221$ & CHE & CLFAN & ALCTA & $X-S E C T$ \\
\hline 1397 & $5446-4321$ & CHEN & CLEAN & $A E C A$ & $x-s+c t$ \\
\hline 1404 & $5446-H 321$ & CHEM & CLFAN & $A L C D A$ & $x=\operatorname{sect}$ \\
\hline 1413 & $5456-4321$ & MILI. & FIN ISH & alena & $x-\sec T$ \\
\hline $14: 4$ & $5456-H \geq 21$ & MTLL & FINISH & $\triangle \mathrm{LCOA}$ & $x=\operatorname{sect}$ \\
\hline 927 & $5456-4321$ & CHFM & CLFAN & ALCenA & $\mathrm{x}=\mathrm{sECT}$ \\
\hline 1394 & $54 a 6-H 321$ & CHEN & CITEAN & nlctis & $x-\sec T$ \\
\hline 1301 & $5456-4321$ & CHEN & CLEAN & $A L C O A$ & $x=\sec T$ \\
\hline 1399 & $5454=4321$ & $\mathrm{CHFN}$ & CLEAN & ALCOA & $x=\operatorname{seC} T$ \\
\hline 1405 & $5456-N 321$ & CHEN & CLFAN & ALCTA & $x-\operatorname{sect}$ \\
\hline 1416 & $S A 4 A=H \geq 21$ & $4 I t, 1$ & FINISH & MLCOA & $\mathrm{X}=\mathrm{FECT}$ \\
\hline 1117 & $545 K=H>>3$ & MTLT & FINSAI & $A\left(C_{1}\right) A$ & $x=5 E C T$ \\
\hline 921 & $535 n=4321$ & CHF:M & $C J=A N$ & NTSOA & $x-\sec T$ \\
\hline $92 A$ & $545 n-1+321$ & CHFN & CI.PAN & $A 1, C) A$ & $X=S F C T$ \\
\hline $13 \times 5$ & $5456-4>21$ & CHEN & CH.FAN & $A I_{1} C^{\prime} \|_{A}$ & $x=3$ \\
\hline
\end{tabular}

GAHNAC ALCOA 47 BARNAC ALCOA 47 GAFMAC ALCOA 47 ALCOA 49 ALCOA 49 BARNAC ALCOA 47 BAANAC ALCOA 47 BAFNAC ALCOA 47 GARNAC ALCOA 47 BARMAC ALCOA 47 ALCOA 66 ALCOA bo GAFAAC ALCOA 4 BAFMAC ALCOA 47 BARNAC ALCUA 47 BARNAC ALCOA 17 GARNAC ALCOA 47 ALCOA b1 ALCOA O? BAFNAC ALCLA 47 GaHNaC alcoa 17 GARNAC AICOA 47 
TABLE It

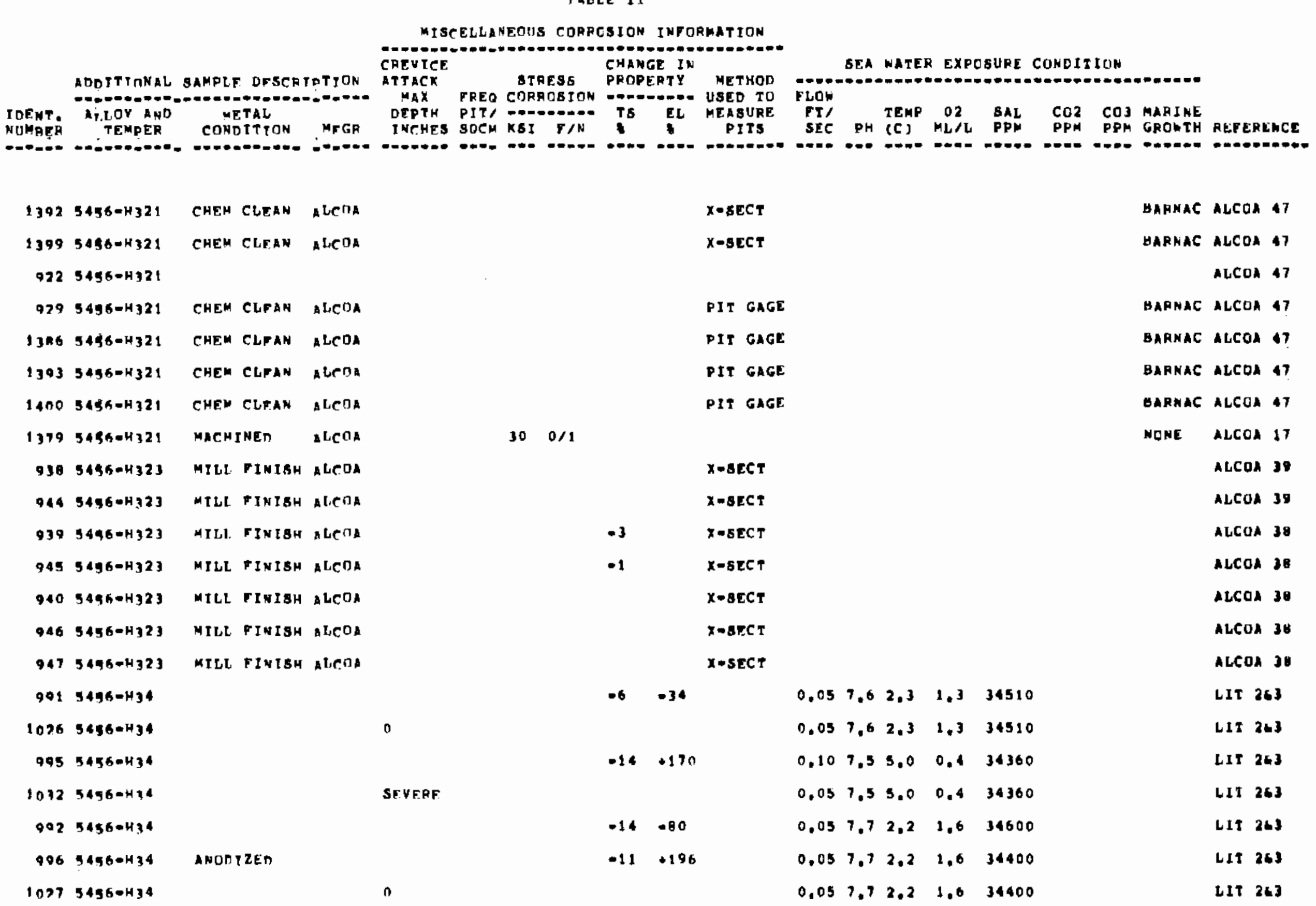


TABIE I I

MTSREMLANEOUS CRRRCBION INFGHMATION

CREVTCr

CREVICF

CHANGE IU

PROPERTY

ATTACK

STRESS

ADRTTITNAL SAUPL OFSCRTDTITN ALtovition netalitin MAY FREA CORRISION -..E.T.E USED TO DEPTH PIT,
THCHES SUCM KST T/N TS EL MEABURE

1 PIT

SEA WATEH EXPOSURE CONDITION

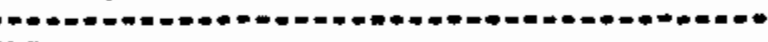

FT' TEMP 02 SAL CU2 CO3 MARINE SEC PH (C) ML/L PPM PPM PPM GRONTH REFERLMCE CINDITTHN MFGR TEMPER -

(2)

NITHF

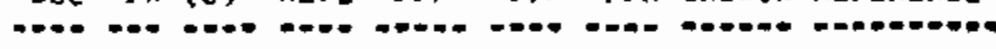

$10285456-434$

$9035456 * \mathrm{H}_{34}$

$10295496-434$ $0045456-H_{34}$

$10305456-434$

$10315456-434$

$9975456-4343$

$9985456-4343$

$10335456=\mathrm{H3} 3$

$10025496 \rightarrow \mathrm{H}_{3} 43$

$10385496 \div 4343$

$10395456=\mathrm{H3} 33$

$9905456=1343$

$1034545 \mathrm{~h}=\mathrm{H343}$

$10355456-13343$

$10005456=+343$

$103654 \% 6=4343$

141月 $545 x=4343$

$\ln 515450-4343$

$1037945 x=4343$

$13745456-4343$

$13755456=4743$

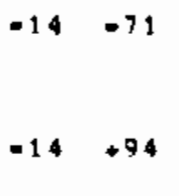

PERF

INC I P

$+10+33$
$0+27$

Ine IP

$-3+22$

INCTD

INC IP

$-2+54$

.028

.029

.054

MTLT FINTSH ALCRA

.035

$n 2$

niti. bintsh mitria

NIIT FIAISH AITRA
LII 3

$0,057,62,3 \quad 1,334510$

LIT 263

$0.057 .62 .3 \quad 1.3 \quad 34510$

LIT 263

LIT 263

LII 263

LIT 263

LIT 263

LIT 263

LIT 263

LIT 26I

L1T 263

LIT 263

[IT 24J

LIt 24]

LII 263

LIT 243

LIT 243

ALCUA 62

LII 243

$4112+3$

ALCUA 17

BARMAC ALCOA 17
$0.057 .52 .0,1.234510$

$x=\operatorname{sect}$

$x+B E^{T} T$ 
TABLE II

MISCELLANEOUS CORROSIUN INFORMATION

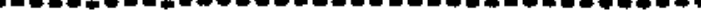
CREVICE

STRESS PHANGE IN

ATraCK

ADEITIONAL SAMDLE DESCRIPTIOH

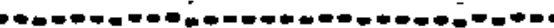

MAX

STRESS

PROPERTY METHOD DEPTH PIT, INCHES SOCM KSI TIN CONDITION MFGR

IDENT,
NUMBFR TOY AN
TEMPER CONDITTON
SEA WATER EXPOSURE CONDITION FLOW FT/ TEHP 02 SAL $\mathrm{CO}$ CQ3 MARINE SEC PH (C) ML/L PPH PPM PPM GROWTH REFEREHCE

\begin{tabular}{|c|c|c|c|c|}
\hline 941 & $5496-\mathrm{H}_{3} 43$ & MTLL & PIN I 84 & $\triangle L C D A$ \\
\hline 948 & $5456-\mathrm{H}_{3} 43$ & NTLL & FINIEH & $\triangle \operatorname{LCOA}$ \\
\hline 424 & $5436-4343$ & MTLL & FINISH & A LCOA \\
\hline 1432 & $5456-\mathrm{H}_{3} 43$ & M I L I I & FINISH & $\triangle \mathrm{LCOA}$ \\
\hline 942 & $54 \$ 6-H_{3} 43$ & MI LL & TINIBH & ateon \\
\hline 949 & $5436-H_{3} 43$ & HILL & FINISH & ALCAA \\
\hline 1475 & $5456-4343$ & MTLL & FINISH & LCOA \\
\hline 433 & $5456-\mathrm{H}_{3} 43$ & 4ILL & FINISH & $\triangle \operatorname{LCOA}$ \\
\hline 426 & $5456-\mathrm{H}_{3} 43$ & $M I L L$ & FINISH & $A L C D A$ \\
\hline 434 & $5436-\mathrm{H}_{3} 43$ & NILL & FIMIBH & alcon \\
\hline 943 & $5456=H_{3} 43$ & MILI. & FINISH & abcoa \\
\hline 950 & $5456 \cdot H_{3} 33$ & MILI & FINI8H & ALCOA \\
\hline 1427 & $5455=\mathrm{H} 343$ & MILL & FIN18H & ALCDA \\
\hline 435 & $5446-4343$ & MILL & FINISH & $A L C O A$ \\
\hline 951 & $5456-H_{3} 43$ & HILL & PINJSH & ALCOA \\
\hline 2428 & $5456-4343$ & MILt & FIN I BH & $A C O A$ \\
\hline 1436 & $3456 \cdot H_{3} 43$ & MILI. & FINIBH & $\triangle \mathrm{LCOA}$ \\
\hline$\$ 420$ & $3456-\mathrm{H}_{343}$ & 4 I LL & FINTSH & ALCTA \\
\hline 1437 & $5456 \cdot 1343$ & MILL & FINISH & ALCDA \\
\hline 952 & $5456 \cdot H_{3} 43$ & MTLL & FIAISH & ALCOA \\
\hline 430 & $5436 \cdot 1343$ & MIILL & FINISH & ALCOA \\
\hline & $\mathbf{5}$ & & INISH & $A \operatorname{LCO}$ \\
\hline
\end{tabular}

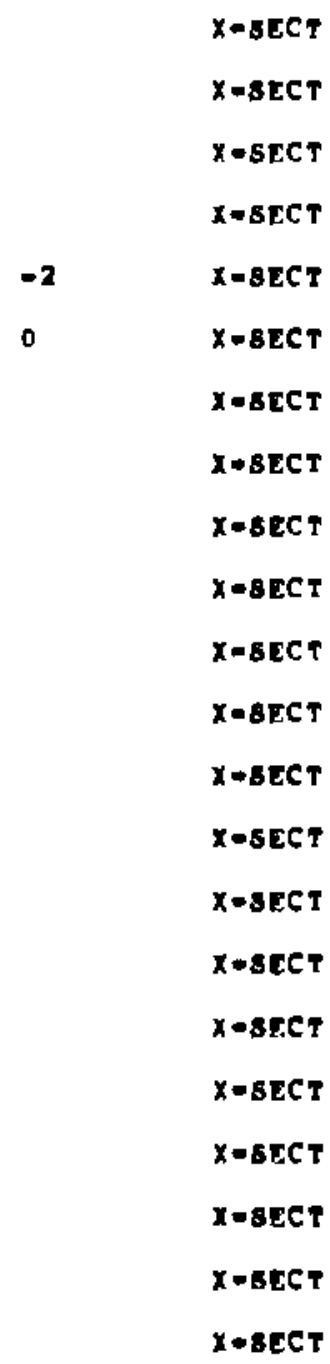

ALCOA 39

ALCOA 39

ALCOA 69

ALCOA 69

ALCOA 38

ALCOA 30

ALCOA 69

ALCOA 69

ALCOA 60

ALCOA 60

ALCOA 38

ALCOA 30

Accot 60

ALCOA 69

ALCOA 38

ALCOA 69

ALCOA 69

ALCOA 60

ALCOA 69

ALCOA 69

alcoh 69

ALCOA 65 
TABIE II

MiscrilanEUUS COPHCSION INFOHATTJUN

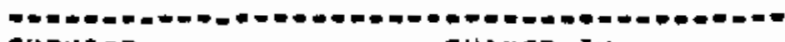

CHEVICE

CPEVICE

CHANGE IN
PROPEATY UETHOD

ANDTTIINAL SAMPTE, CFSTRTDTTIOH TTACK STRESS PROPEATY GETHOD TDENT ALTOY AND

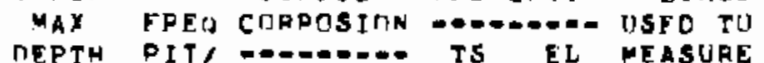

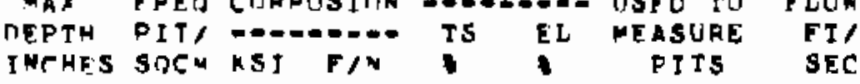

SEA WATEK EXPOSUPE COMDITIUN 1 ALTY AND METE
TEHDER CONDITITN MFGR INTHES SHCa RST FIN PITS

FLOW
FI'
SEC

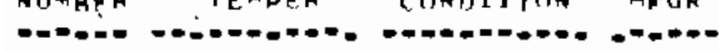

\begin{tabular}{|c|c|c|c|c|c|}
\hline 1471 & $5456=4>43$ & $4 \mathrm{ILI}$ & FINISH & $\triangle \mathrm{LCOCA}$ & $x=\operatorname{sfct}$ \\
\hline 1376 & $5456-5>43$ & MIJI. & $F[\operatorname{NISH}$ & $A$ L.COA & $x=S E C T$ \\
\hline 1406 & $5456=\pi$ & $M P 1, \mathrm{~L}$ & FIN TSH & ALCOA & $x$-sect \\
\hline $142 n$ & $54: 6=n$ & $M T L t$ & FINISH & $A \operatorname{LCnA}$ & $x=5 F C T$ \\
\hline 1422 & $5456-n$ & MIL. & FINIS4 & aLCtha & $x=\operatorname{SEC} T$ \\
\hline 1409 & $5 A Q 6=0$ & 471.1 & FINISH & A. & $x=\sec t$ \\
\hline 1412 & $54 \times 6=n$ & 4 ILI. & FINISH & atens & $x=S E C T$ \\
\hline $14+5$ & $5495=n$ & 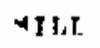 & FINISH & $\triangle L C \cap A$ & $x=\operatorname{sect}$ \\
\hline 915 & $5457=K 34$ & ACET & CITFAN & DEY & MICROS \\
\hline 918 & $5497-\mathrm{H} 34$ & ACET & CHEAN & DEY & MICROS \\
\hline 916 & $5457-434$ & ACFT & ClFAN & $P E Y$ & MtCars \\
\hline 010 & $5497-H_{34}$ & ACFT & CLFAN & EE:Y & HICRUS \\
\hline 917 & $5457-N 34$ & $\operatorname{ACET}$ & CLFAM & $-3,1-2,5$ & merusc \\
\hline 020 & $5457-\mathrm{H}_{34}$ & ACF' & CITAN & $-4.3-16$ & MICPNBC \\
\hline R21 & $B \cap Q 1=T 4$ & & & $B L C A N$ & \\
\hline 925 & 6na1-TA & & & atran & \\
\hline A 45 & $\sin 1-74$ & & & $\triangle L C A N$ & \\
\hline 940 & $\operatorname{nn} 51=T A$ & & & $\triangle I, C A N$ & \\
\hline Q)? & $6 n a 1-\pi 1$ & & & MirAli & \\
\hline $9>5$ & BOA1-T4 & & & a. & \\
\hline DAS & $\operatorname{hng} 1=T 4$ & & & $\operatorname{lic} \Delta N$ & \\
\hline 9al! & $m\left\{A_{1}=T_{1}\right.$ & & & alicas & \\
\hline
\end{tabular}

LCOA 69

NGRE ALCUA 17

ALCOA 48

ALCOA 24

ALCOA 29

ALCOA 49

ALCOA 60

ALCOA 67

DARNAC LIT

BARNAC LIT 4

BAHNAC LII

BAHNAC LIT 4

GARNAC LIT 4

BAFNAC LIT 4

LI' 9

LII 9

ᄂ1工

L1T

LII 9

4119

LI1 9

LII 9 
TABLE II

MISCELANEOUS CORACSTON INFORMATION

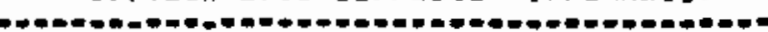

CHANGe In

CREVICE CHANGE IN

ATTACK STRESS PROPERTY METHOD

ADEITITNAL SAHPLE DEBCRTPTTIN

USED TO

OEPTH PIT/ C..... TS EL MEABURE

IHCHES SOCM KSI T/N MITS

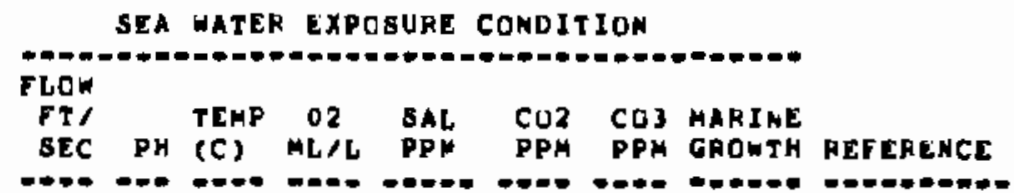

NUHBER

TEMPER

METAL

MVGR

INCHES BOCM KSI PITS SEC PH (C) ML/L PPM PPM PPM GAOWT

$6236051-74$

927 $6051-74$

$0476051=T 4$

Q51 $6051=74$

$024609 \mathrm{t}-\mathrm{T} 4$

828 6051-T4

$8486091=T 4$

$0526051=T 4$

$0376051=74$

$6416091-T 4$

$9076051+14$

$9116051-74$

Q3月 $6081=T 4$

$0426031=T 4$

$9086051-T 4$

$9126051-14$

$8396054=74$

$0436031-74$

$9096051-14$

$9136051-74$

$\$ 40$ 6ng1-T4

$0446051=T_{4}$
ACAN

LCAN

atch

a LCAN

ALCN

a LCAN

a LCAN

SLCAN

I LCAN

ALCAN

ALCAN

LCAN

ALCAN

ICAN

aLCAN

A LCAN

A LCAN

ALCAN

SUCAN

LCAN

AICAN

ALCA
WIT 9

LIT 9

LII 9

以【 9

LII 9

LIT 9

4IT 9

LII 9

LIT?

LIT

LIT 9

LII 9

LIT 9

LIT

LIT 9

LIT 9

4II

LIT

UIt

LI

LIT

LIT 9 
TAELE II

MISCELLANEOUS CORACSION INFOPMATITN

CDEVICE

CQEVICE
ATTACK SHANGE IN

ADATTIONAL SAMPLF RFSCRTTTTUN METKRD

DEPTH PIT, OA.0.-. TS EL MEASURE

SEA WATER EXPOSURE CONDITIDA

AL LY AND

$\operatorname{TDENT}$ METAL INCHES SOCM KSI $\mathrm{F} / \mathrm{N}$ TS EL MEASURE $\begin{array}{lllll}\text { FLOK } & \text { TEMP } 02 & \text { SAL } & \text { CQ2 CU3 MAPINE } \\ \text { SEC PH (C) ML/L PPM PPM PFM GROMTH REFERENCE }\end{array}$ NUMREF TEMPER COMDITITN HEGR

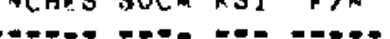

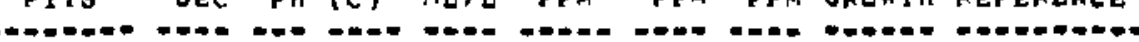

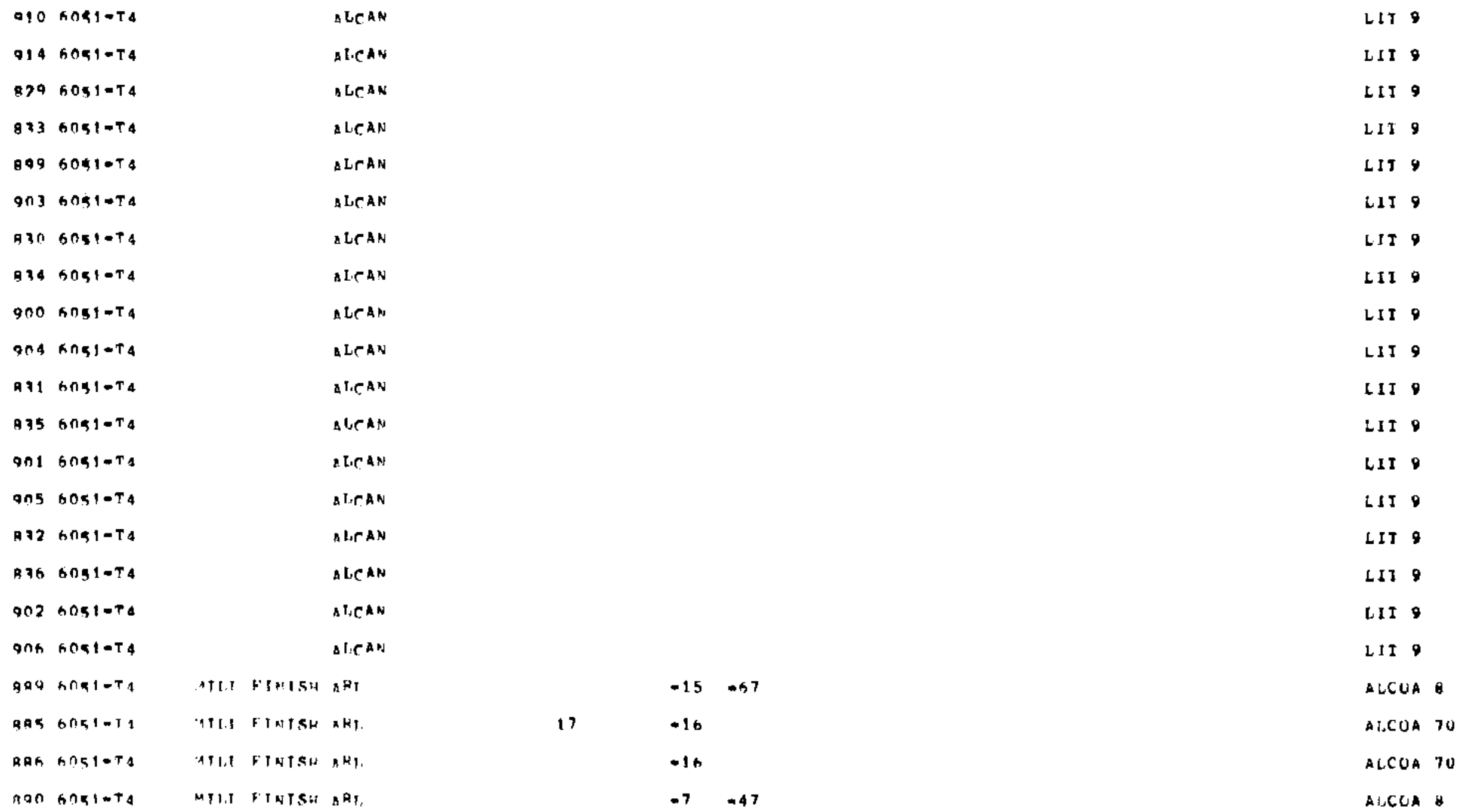


TABLE II

MISCELLANEOUS CORPCBION IHFORMATION CHEVICE

CAEVICE STAESS PHANGE IN

ADATTCNAL SAMPIE DESCAIDTION

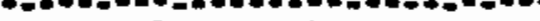

MAX PREO CORRDBIDN ...... USED TO DEPTH PIT, -OE.E.E. TS EL MEASURE

INCHES SOCN KSI TIN PITS

SEA WATER EXPOSURE CONDITION
FLOW

IDENT,

METAL
TEMPER CONDITION MFGR

FLOW

TEMP 02 BAL CO2 COJ HARINE

INCHES SQCN KS

PITS SEC PH (C) ML/L PPM PPM PPM GROHTH REFEKENCE

\begin{tabular}{|c|c|c|c|}
\hline 891 & $6051-74$ & MILL FINISH & $\triangle A L$ \\
\hline 892 & $6051-74$ & MILL FINIBH & $\mathbf{A} \mathbf{R}_{\mathbf{I}}$ \\
\hline 813 & $6051+T_{h}$ & & ALCAN \\
\hline ค17 & $6051-76$ & & ALCAN \\
\hline 869 & $6051-76$ & & ACAN \\
\hline 873 & $6051-76$ & & LCAN \\
\hline 014 & $6051=76$ & & ALCAN \\
\hline 918 & $6071-76$ & & LCAN \\
\hline 970 & $6051=76$ & & ALCAN \\
\hline 974 & $6051 \cdot 76$ & & ALCAN \\
\hline 815 & $6051=+6$ & & ALAN \\
\hline 949 & $6051=76$ & & LCAN \\
\hline 871 & $6051=76$ & & aLCAN \\
\hline Q75 & $6051-T 6$ & & LCAN \\
\hline 816 & O051-Th & & $D C_{A N}$ \\
\hline 870 & $6051 \cdot 76$ & & LEAN \\
\hline B72 & $6091=76$ & & ALCAN \\
\hline R76 & $6091-76$ & & atern \\
\hline An & ons1-Th & & ALCAN \\
\hline 010 & 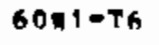 & & ALCAN \\
\hline 861 & bnsi-Th & & aLCAN \\
\hline 865 & $6051 \cdot T h$ & & ALCAN \\
\hline
\end{tabular}

LIT 9

LIT 9

L15

LIT 9

LI) 9

LIT 9

LIT 9

LIT 9

LIT

LIT 9

LIT 9

LIT

LIt?

LIT 9

LIT 9

LIT 9

LII

415 9

LIT O

LIT 9 
TABIE. II

MTSCFLLANERUS CORECSION INTCRMATIUN

CREVICT
MTPACK STRESS PROPFRTY NFTHOD

ARITTIMNAL SAMPIE DFSCRTDTTON

MP

MAX FREO CTRRUSITN -..... USED TD

DEPTH PIT, PITS

SEA MATER EXPOSURE CONDITIGN

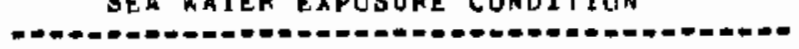

FLOW

FT' TEMP 02 SAL CO2 COJ MARINE

IHCHES SOCA KSI F/N

SEC PH (C) ML/L PPM PPM PPM GROWIH REEERENCE

NUMAFP TEMPFR CONDITTON MFGR

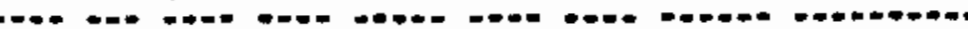

\begin{tabular}{|c|c|c|}
\hline $\operatorname{A\cap } 7$ & SOS1-Th & $\Delta \operatorname{LCAN}$ \\
\hline $\operatorname{san} 2$ & $5051=T A$ & $A L C A N$ \\
\hline 866 & ansi-ta & $A L C A N$ \\
\hline $\operatorname{gn} x$ & $60 \mathrm{~g}=\mathrm{Th}$ & DICAN \\
\hline ค11 & $\tan 1-\pi 6$ & $\triangle L C A N$ \\
\hline 963 & 6051076 & $\triangle \mathrm{LICAN}$ \\
\hline 967 & $60 \times 1-7 \mathrm{~h}$ & a tic AN \\
\hline and & SOS1-Th & $\Delta L C A N$ \\
\hline ค1 2 & $6 \cap 51-T_{6}$ & $A \operatorname{CA} A$ \\
\hline D.4 4 & $6051-T_{6}$ & ALCA \\
\hline REA & hOST-T 6 & $\Delta \mathrm{t}, \mathrm{C} \wedge \mathrm{N}$ \\
\hline 893 & SOS1 $=T G$ & ALAN \\
\hline 987 & AOE 1-TA & a LCAH \\
\hline 877 & GOS1-Th & ALCAN \\
\hline RคI & $0051=76$ & ALCAN \\
\hline B5 4 & GOS:-th & $A \operatorname{LCAN}$ \\
\hline $\operatorname{sen} \theta$ & GOS1-Th & LCAN \\
\hline R? & hOS $1=T A$ & ICAN \\
\hline AR 2 & $\operatorname{nnc} \uparrow-T_{n}$ & $A L C A N$ \\
\hline $9 \$ 5$ & 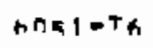 & al, $\mathrm{CAn}$ \\
\hline 950 & $h \cap K 1=T_{A}$ & $A=C A B$ \\
\hline 879 & $h \cap 51=T h$ & $A \operatorname{IrCAN}$ \\
\hline
\end{tabular}

L I T

LIT 9

1 II 9

LIT 9

LIT 9

LIT 9

LII 9

LIT 9

LIT 9

LII 9

LIT 9

LIT 9

1129

LIT 9

LII 9

แโ 9

LII 9

LII 9

LIT 9

LIT $y$

$11 T y$

LII 9 
TABLE II

MISCELLANEOUS CORACSION INPOAMATION

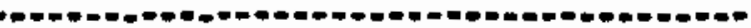

CREVICE

CAEVICE

STPESS PHANGE IN

METHOD ADDTIONAL SAMPLE DFSCRIFTION

STRESS PROPERTY

METHOD
USEO TO

DEPTH PIT,

SEA WATER EXPOSURE COMDITIOM Gena mater Exposuke comdition IDENT, ALLOY AND METAL
NUMEER TEMDER CONDITION MFGR TEMP 02 SAL COZ COJ MARINE

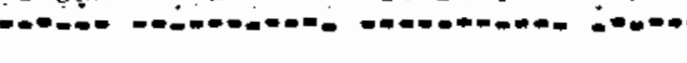
PIT

\begin{tabular}{|c|c|c|c|c|c|c|c|c|c|}
\hline ค9 3 & $6051 \cdot 76$ & & & ALCAN & & & & & \\
\hline ค5 6 & $6051=T h$ & & & ALCAN & & & & & \\
\hline$B 60$ & $60 \$ 1=T A$ & & & ALCAN & & & & & \\
\hline 8月0 & $6051=76$ & & & ALCAN & & & & & \\
\hline 644 & $6051-76$ & & & ALCAN & & & & & \\
\hline 993 & $6091-76$ & MILL & FINI8H & ${ }_{A} P_{t}$ & & & .24 & -87 & \\
\hline 894 & $6051=75$ & MTLL & FINISH & ${ }_{A} P_{L}$ & 34 & $0 / 1$ & -9 & -87 & \\
\hline ดด 7 & 6051076 & MILL & FINISH & $A_{L}$ & 37 & & -25 & & \\
\hline ดค 8 & $60 \$ 1-T_{6}$ & MTLL & FINISH & $A R \mathbf{L}$ & & & -31 & & \\
\hline 095 & Gns1-Ta & MILt & FINISH & $\mathbf{R}_{\mathbf{L}}$ & & & .11 & .77 & \\
\hline 896 & $5051=T_{5}$ & MtLL & FINISH & $A R t$ & 34 & $0 / 1$ & -10 & -81 & \\
\hline 897 & $0051-\mathrm{Th}$ & MILI. & FINISH & $A R L$ & & & -10 & .19 & $x-\sec t$ \\
\hline 998 & $6 \cap 91=56$ & HILL & FINISH & $A R_{L}$ & & $0 / 1$ & & & \\
\hline 1458 & $6053-14$ & & & $\triangle L C O A$ & & & -6 & -50 & \\
\hline 1454 & $605 x+74$ & & & ALCHA & 17 & $0 / 2$ & 0 & & \\
\hline 1455 & $6053 \cdot \mathrm{T} 4$ & & & ALCOA & & & 0 & & \\
\hline 1459 & $6053=14$ & & & $\operatorname{sLC} A$ & & & -16 & -62 & \\
\hline 1460 & $50,3-T 4$ & & & $\sec x$ & & & -1 & -6 & $x=$ sect \\
\hline 1461 & 6ก53-T 4 & & & Atcon & & $0 / 2$ & & & \\
\hline 1440 & $6053=\mathrm{T} 6$ & & & Alcos & & & -0 & & \\
\hline 1441 & $6053=76$ & & & $\operatorname{AlcOA}$ & & & -4 & & \\
\hline 1442 & $6053-T 6$ & & & ALCOA & & & .39 & & \\
\hline
\end{tabular}

LIT 9
LIT 9
LIT $\$$
LIT 9
LIT
ALCOA
ALCOA
ALCOA 70
ALCOA 70
ALCOA
ALCOA 0
ALCOA 9
ALCOA
ALCOA
ALCOA 10
ALCOA 70
ALCOA
ALCOA
ALCOA
ALCOA 16
ALCOA 10


TARTE II

MISCELLRAEOUS CARHCSION INFTIRAATION

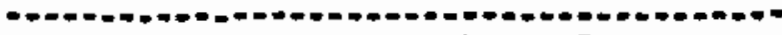

CPEYTCE

CPEVICE CHANCE IN MTRES PRT

ADAITITNAL SANPIE RESCRTPTITN TTACK

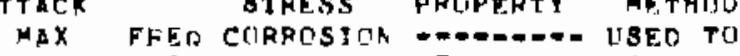
ALTU

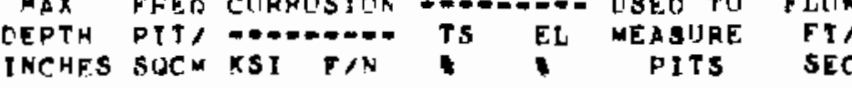

SEA WATEH EXPESUFE CONDITIUN COCH. FLOW

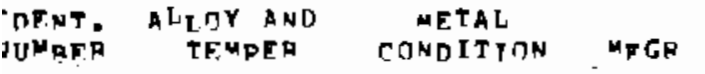

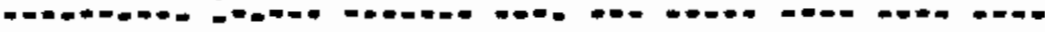

\section{$14436 \cap 53=T 6$}

769 6053-16

$7716 \cap 53-T 6$

798 hก:3-T

$7026053=T h$

$7686043=16$

$7126053=T$

7R0 hO43-T6

$7036093=1$

Th9 GnE 3-Th

$773 n 0 a 3=T$

700 hOQ3 3 Th

794 6n53-T

770 6053-Th

$701505 x=t 6$

764 ค0 3-T6

7R) bกद 3-Th

70: AnE?-Th

an) fricasert

7 as aredoth

7ค2 1:- 21075

$7 n !, 15 ?-1 n$ i Lena

a LCAN

$\triangle \operatorname{BCAN}$

aLCAN

SLCAN

A I,CAN

Al,CAN

L.CAN

ALCAN

a LICAN

I LCAN

DLCAN

a bean

ABCAN

$\triangle$ LCAN

IICAN

SLCAN

A.rAN:

MTAMAI

RICAN

i.

Al.rAt.
ALCUA 16

LIT 9

LI 9

LII 9

LIT 9

LI 9

LIT 9

LIT 9

LIT 9

LIT 9

LI 9

LIT 9

LIT 9

LIT 9

CIT 9

LIT 9

4IT 9

LII 9

I I 9

L1I

LIT 9

LIT' 9 
TABLE II

MIBCELLANEOUS CORROSION INFORMATION

CDEVICE

CDEVICE CHANGE IN

ADOITIOHAL SAMPLE DFSCRIPTION ATTACK TRE BTRESS PROPERTY METHOD

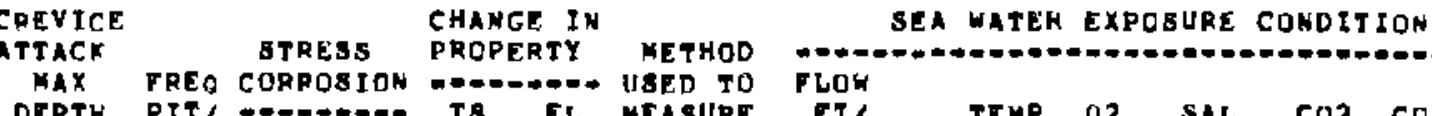

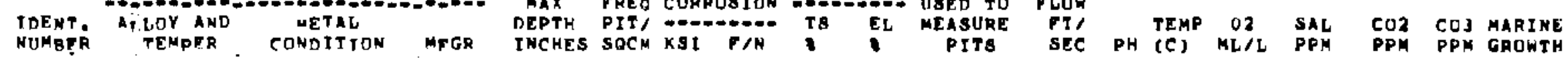

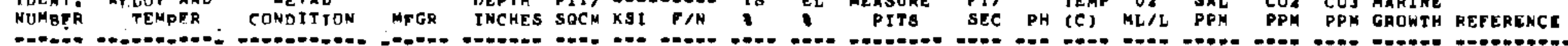

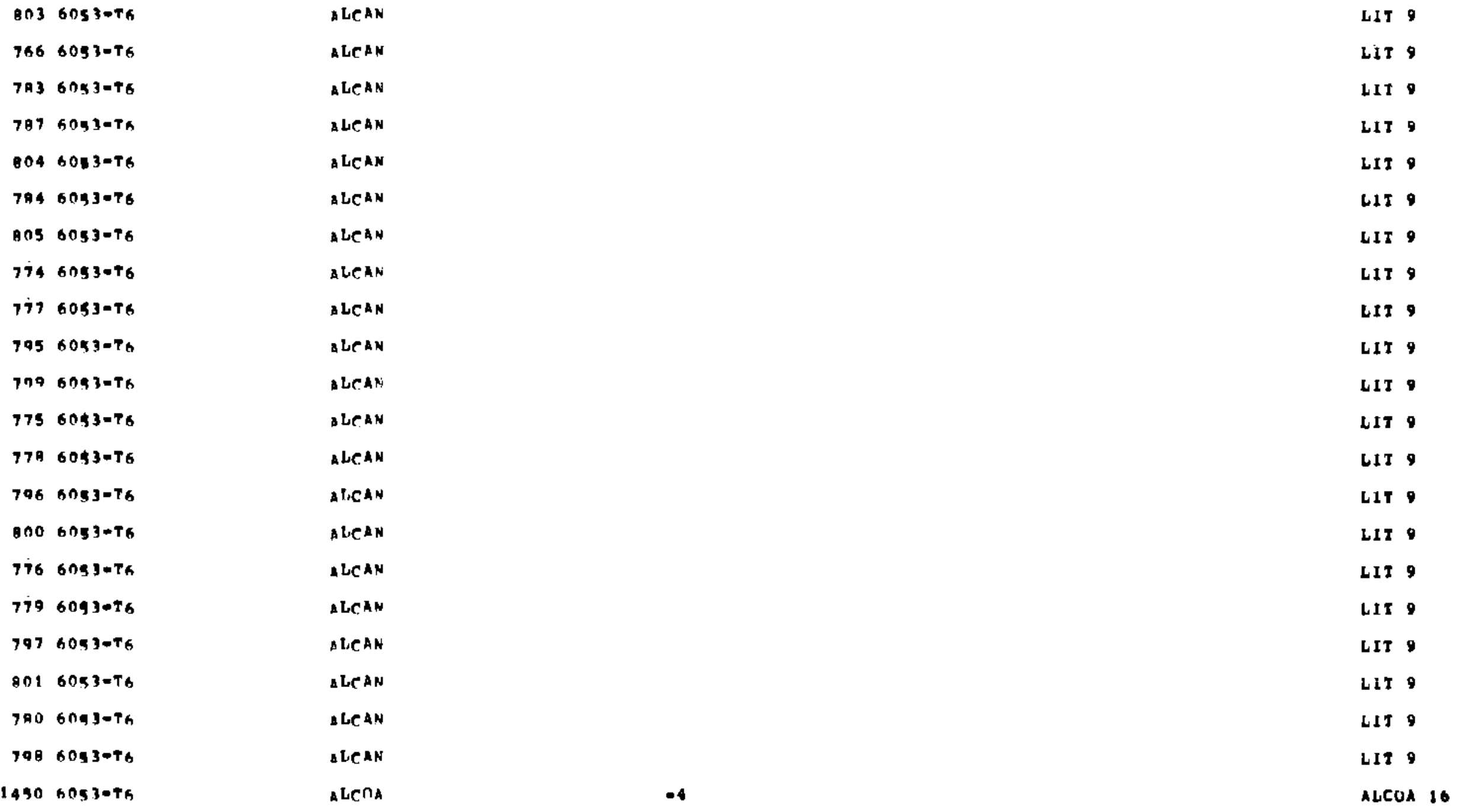


TAFIE II

MSCFILLANEUUS CORPGSION TNFORMATION

COEVICF. CHANGE IN

ATTACK STPESS PROPERTY MFTHOD

ARATTINNAL SANPIF DFSCRTDTTON

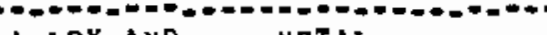

MAX FREO COD

DEPTH PIT, -.+... TS FI MEASURE FT/ TEMP 02 SAL CUZ COJMARINE

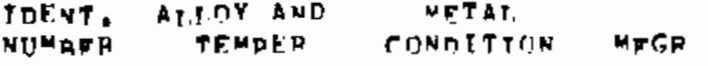

SEA WATEF EXPOSURE CONUITIUN

UMa

TEMPED CON

INCHES RQCM KSI FIN I O PITS SEC DM (C) HI/L PHM PPM PPM GRONTH

1451 anS3-TG

AI, COA

$+2$

$14536053-75$

a IICA

FLC. W

1452 6093-TA

I.COA

.0030

FLOW

ALCOA 16

$\triangle \mathrm{LCT}$

LCHA

A Leria

A LCF A

ALCTA

a LeTA

21.001

nTCOAS

$B \mathrm{I}^{\mathrm{C}} \mathrm{\cap A}$

\begin{tabular}{|c|c|c|c|c|}
\hline & & -7 & .59 & \\
\hline 26 & $0 / 2$ & -6 & -59 & \\
\hline \multirow[t]{3}{*}{23} & $0 / 2$ & $-B$ & & \\
\hline & & -6 & & \\
\hline & & -5 & -39 & \\
\hline \multirow[t]{3}{*}{26} & $0 / 2$ & -4 & -27 & \\
\hline & & -3 & -6 & $x-S E C t$ \\
\hline & $01 / 2$ & & & \\
\hline
\end{tabular}

AlcuA is

ALCOA B

ALCOAO

ALCUA TU

ALCOA 70

ALCLA

ALCLA B

ALCOA $O$

A LCUA

ALCOA IO

A.CDA

abena -3

a LetiA

$-3$

ALCD 10

ALCOA 16

ALCOA 16

Ateria

$-1$

ALCUA 10

ALCOA

ALCOA 16

SITIA

$-1$

$+4$

A. COA 16

aliena

$-11$

ALCUA It 
TAALF II

MISCELLANEOUS CORAOSION INFURHATION

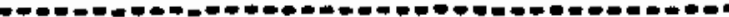

CPIVE

CoEviCE

STRESS CHANGE IN

PRDPEATY METHOD

ADDTTIONAL SAMPLE DFSCRTPTIOH

Track

STRESS

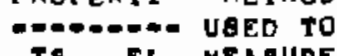

DEPTH PIT,

INCHES SOCM KSI T/N

SEA WATER EXPOSURE CONDITION

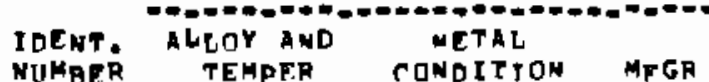

FI' TEHP 02 SAL CU2 CO3 MARINE

$\begin{array}{llllll}\text { FI } & \text { TEHP } 02 & \text { SAL } & \text { CU2 CO3 MARINE } \\ \text { GEC PH (C) ML/L } & \text { PPH PPM PPM GAOHTH REFERENCE }\end{array}$

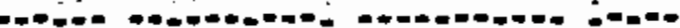

PERT

LII 263

$14696061=$

VAPQR DFG

$<1$

LIT 1

$15416 n 41-T$

VAPCA ORG

$<1$

LII I

$15426081-T$

VAPOR OEG

$<1$

LIT I

$15436061=7$

VAPOR DEG

$154460 \% 1=T$

VAPOR DEG

1545 6061-T

VAPOR DFG

LIT 1

41

LIT 1

$6376041=\mathrm{T} 4$

ILCAN

$<1$

LIT 1

$6366051=74$

ALCAN

LIJ 9

$6196061=74$

ALCAN

$-.5 \quad-6.7$

LII 9

$6406061-74$

ALCAN

$+1-4,6$

LIT 9

UI?

ALCAN

LIT

LCAN

CIT 9

ALCAN

LIT 9

ALCAN

LIT 9

a ICAN

11 I

aLCAN

LI 9

ALCAN

LIT 9

ALCAN

LIT 9

ALCAN

LIT 9

a LeAN

U17?

$6616061=74$

ALCAN

LIT 
TAELE. I I

MISCFLLANEOUS CORHCSION THFORMATION

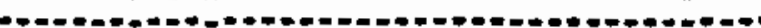
CPEVICF. CHANGE IN CREVICF. AORITIONAL SAMPIF: DFSCDTOTTUN STRESS FROPERTY METHDD

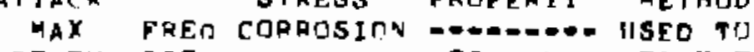
OFPTH PIT, -....... Ts F.L MFRSURE IHCHFS SQCW KSI F/N

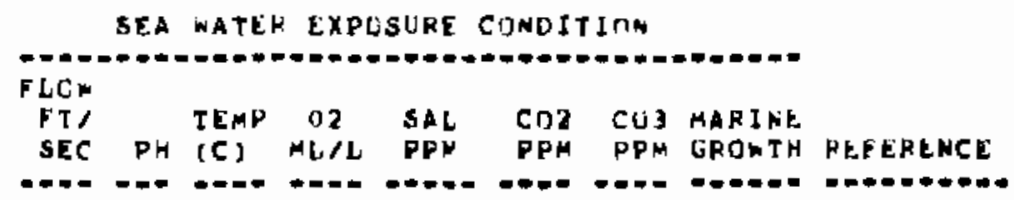

\begin{tabular}{|c|c|c|}
\hline 662 & $6061=74$ & $\triangle G C A N$ \\
\hline 631 & $60 \times 1=T_{4}$ & ALCAN \\
\hline 653 & GBA: $=T_{4}$ & $\triangle \operatorname{ClCAN}$ \\
\hline 632 & SOA1-T4 & $\triangle L C A N$ \\
\hline 664 & GOB $1=T 4$ & $\triangle L C A N$ \\
\hline 647 & $6051=74$ & $\triangle \operatorname{LCAN}$ \\
\hline $6 \times 9$ & bOS1-T 4 & $\triangle L C A N$ \\
\hline 559 & GOBSTA & $B L C A K$ \\
\hline 670 & $00 \times 1-T 4$ & $\triangle L C A N$ \\
\hline 559 & $a_{1} \cap_{6} 1-T_{4}$ & ALCAN \\
\hline 671 & $50 \times 1=T 4$ & A D.CAN \\
\hline$b 6 B$ & GOE1-Ta & $\triangle \operatorname{LCAN}$ \\
\hline $47 ?$ & $6 \cap \times 1-T 4$ & $A \operatorname{LCAN}$ \\
\hline & $n_{n} n_{1}=T_{4}$ & \\
\hline
\end{tabular}

$152 R$ GOGPTH

1 ดी

760 GOK $1=14$

$7 B 1 \operatorname{LOF} 1-T_{4}$

1919 SOALT 4

1 म $20 \quad+1010 T_{A}$

1971 RחE $1-T_{4}$

$1523 \quad h \cap G 1=T A$

1524 GOASTET
MILL FTNISH APT

WITL FIMISH ART

MITI. FINISH ARI.

NTIT VINTSA ART

$\because 11, F[+15 H, H F$

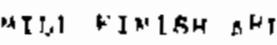

sicis

a. 1.13 MITS SEC PH (C) MLL PPN PPM PPM GROTH PEEERECE

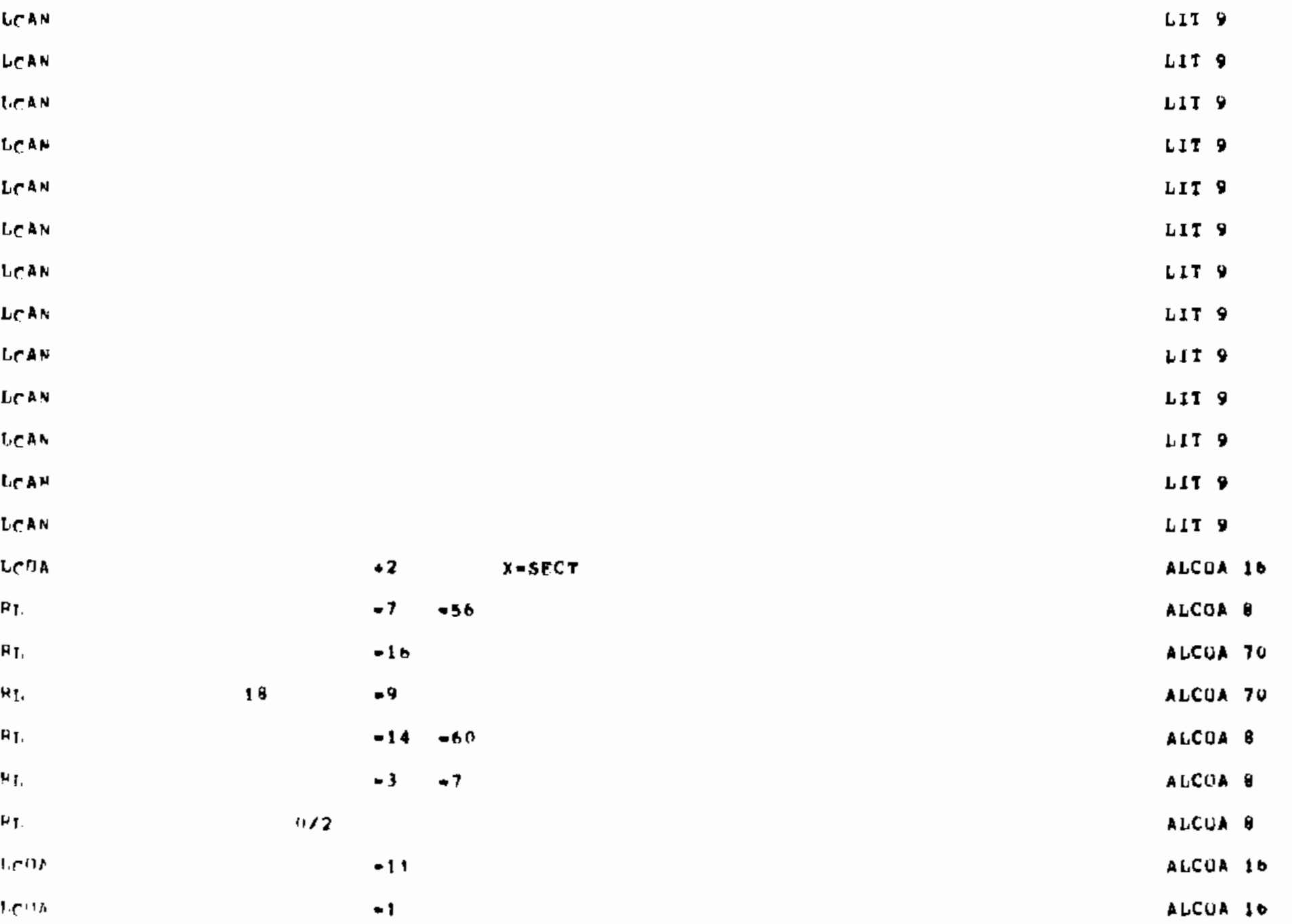


TABIE It

MISCFLLANEOUS CORRCSION INTORMATION

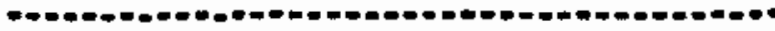

CREVICE

CREVICP

CHANGE IN

PROPERTY METHOD

ADDITIOMAL SAMPLE DFSCAIPTTON

TTACK

CROPERTY METHOD

DEPTH PIT/ TIE TS EL MEASURE

SEA WATER EXPOSURE CONDITION

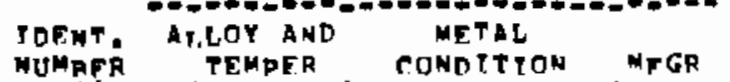

INCHES SOCM KSI FIN H PITS SEA WATER EXPOSURE CONDITIOK

PIS TEMP O2 SAL COZ COJ MARINE

$\begin{array}{llllll}\text { PT' TEMP OZ } & \text { BAL } & \text { COZ CO3 MARINE } \\ \text { SEC PH (C) ML/L PPM PPM PPM GROHIH REFEREMCE }\end{array}$

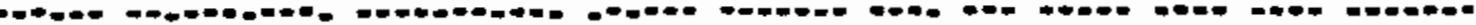

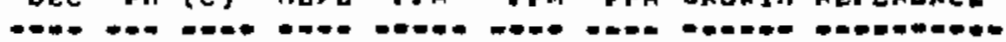

\begin{tabular}{|c|c|c|}
\hline 653 & 6061-T6 & LCAN \\
\hline 654 & $6061-96$ & ALC \\
\hline 655 & $6081=76$ & Ale \\
\hline 656 & $60 \mathrm{AS}-\mathrm{T} 6$ & abe \\
\hline 1521 & $6061=16$ & $\triangle L C$ \\
\hline 649 & $6081=76$ & Lcc \\
\hline 693 & $6061-T h$ & ALC \\
\hline 697 & $6064=T 6$ & ALC \\
\hline 701 & $6061-T 6$ & LCC \\
\hline 650 & GOB 1-TA & $\triangle L C$ \\
\hline 694 & SOA1-TA & $\triangle L C$ \\
\hline 690 & $6041=76$ & ALC \\
\hline $7 \cap 2$ & 60G1-TA & LCC \\
\hline 651 & $6061-76$ & ALC \\
\hline 695 & 60A1-T6 & $\Delta L C$ \\
\hline 699 & $606 t=T 6$ & $\triangle L C$ \\
\hline 703 & $6061-76$ & ALC \\
\hline 692 & GOA1-TA & $A], C$ \\
\hline 606 & 6ns:-7s & AldC \\
\hline $7 n 0$ & SOAS-Th & al, \\
\hline 704 & Gns $1=76$ & shed \\
\hline 522 & $6081=7$ & a of \\
\hline
\end{tabular}

LEAN

LII 9

LIT 9

LIt 9

LIT 9

alcon 15

LIT 9

4179

LIT 9

LIT 9

LIT 9

LIT 9

LIT 9

LIT 9

LIt

LIT 9

LIT 9

LIT 9

LIT 9

LII 9

LIT 9

LIT 9

AlCoA 14 
TABLE II

MTSCFLLANEOUS CTRHCSION INFOHMATION ADRITIONAL SANPLF. DESCRTOTION InFNT. ATIOYY ANO Crivice CRPVICE CHANGE IN

ATTACK STHESS PPOPERTY METHOD

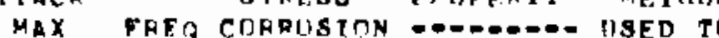
TAPTH PIT/

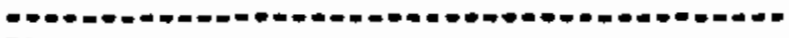
FLOW TEMY 02 SAI CO2 COJ MARIME FI/ TEMP 02 SAI CO2 COJ MARINE SEC PH (C) ML/L PPM PPM PPM GROTIH KEFERENCE -

\begin{tabular}{|c|c|c|}
\hline 645 & $A_{0} \cap 61-T G$ & $\triangle \mathrm{LCAN}$ \\
\hline 691 & GnG $1=T h$ & $\triangle L C A N$ \\
\hline GRS & $B \cap A 1=T B$ & $A \mathrm{LCAN}$ \\
\hline 699 & Gnat-Ta & ALCAN \\
\hline 646 & SnAl-Th & ALCAN \\
\hline 622 & $G D A 1=76$ & $A^{1}$ CAN \\
\hline $6 R 6$ & hos $1=T h$ & $A \operatorname{lic} A N$ \\
\hline 600 & $A_{n} A_{1}=T_{6}$ & $A T / C A N$ \\
\hline 447 & $60 \times 1-76$ & $A \operatorname{tr} C A N$ \\
\hline SR3 & GOSG & $\triangle L C A N$ \\
\hline 607 & GnA $1=T_{A}$ & $A T H A N$ \\
\hline 601 & $6 n x_{1-T h}$ & $\triangle L C A N$ \\
\hline 648 & $6061-76$ & $A \operatorname{LCAN}$ \\
\hline 69 4 & $A_{B} \cap_{A}=T_{B}$ & ALAN \\
\hline 6月B & KOKI-Th & $\triangle L C A N$ \\
\hline 592 & $6 n 6 t=T h$ & $A T C A N$ \\
\hline 641 & $60 A_{1}=T_{A}$ & $\triangle A C A N$ \\
\hline 673 & AnA1-Th & $\triangle L C A N$ \\
\hline 677 & hnal-Th & AlirAx. \\
\hline 372 & mox $1=7 h$ & DLCAN \\
\hline 647 & SOKA $=T S$ & $\Delta 1$, C $! H$ \\
\hline 674 & $h \cap n 1-T h$ & $B \in C A D$ \\
\hline
\end{tabular}

L1T

LII 9

LIT 9

LIT 9

LIT 9

LIT 9

LI T O

LIT 9

LI' 9

LIT 9

LIT

LIT 9

LIT?

UII 9

LIT 9

LIT 9

LIT 9

LII 9

LIJ 9

LIT 9

LIT 9

LIT 
TAALF, II

MIBCELLAREUUS CORROSION INFORMATIOA

CDTVICE

CaEvice

STRESG CHANGE IN

PROPERTY METHOD

ADITITNAL SAMPIE OFSCRTPTYON

TTACK

STRESS

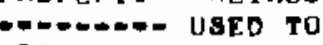

DEPTH PIT,

INCHES SOCM KSI FIN

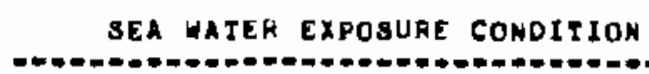

PLON

FLOW TIE O2 SAL CO2 COJ MARINE

TOENT.
MUMRER

NETAL
CONDTTTN

HFGR

PITS SIT
PITS

$\begin{array}{lcccc}\text { TEMP } & 02 & \text { SAL } & \text { CO2 } & \text { CQ3 MARINE } \\ \text { (C) } M L / L & \text { HPM } & \text { PPM PPM GROWTH }\end{array}$

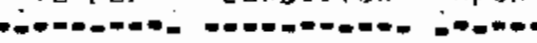

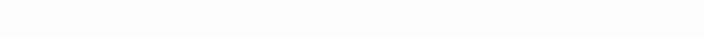

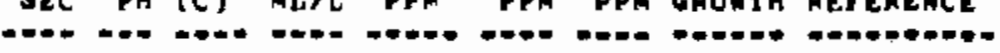

\begin{tabular}{|c|c|c|c|c|}
\hline S78 & SOA1 $=7 h$ & & ALCAN & \\
\hline 733 & $6 n \times 1=76$ & & $\triangle L C A N$ & \\
\hline 643 & $6061=76$ & & ALCAN & \\
\hline 675 & 60A1-Th & & ALCAN & \\
\hline 679 & $6081 \cdot 76$ & & ACAN & \\
\hline 734 & $6061-T 6$ & & $A L C A N$ & \\
\hline 644 & $6061-75$ & & LCAN & \\
\hline 676 & $6061+76$ & & $\triangle L C A N$ & \\
\hline 670 & $6091-76$ & & ALCAN & \\
\hline 735 & $6061=76$ & & ALCAN & \\
\hline 1472 & Snst $=T h$ & & & \\
\hline 1473 & $60 \times 1-T 6$ & & & .010 \\
\hline 1474 & $6061-56$ & & & \\
\hline$\$ 475$ & 6041-Th & & & .032 \\
\hline 1476 & $6061-7 h$ & As WELDRn & & 0 \\
\hline 1477 & 60A1-TS & H.T. T T6 & & 0 \\
\hline 1479 & AOSI-Th & & & .021 \\
\hline 1479 & $60 \times 1=74$ & & & \\
\hline 1480 & 6061-Th & & & 0 \\
\hline 1481 & $6051=T h$ & & & .032 \\
\hline 1511 & $60 \times 1-56$ & & & \\
\hline 1512 & $60,1-76$ & & & \\
\hline
\end{tabular}

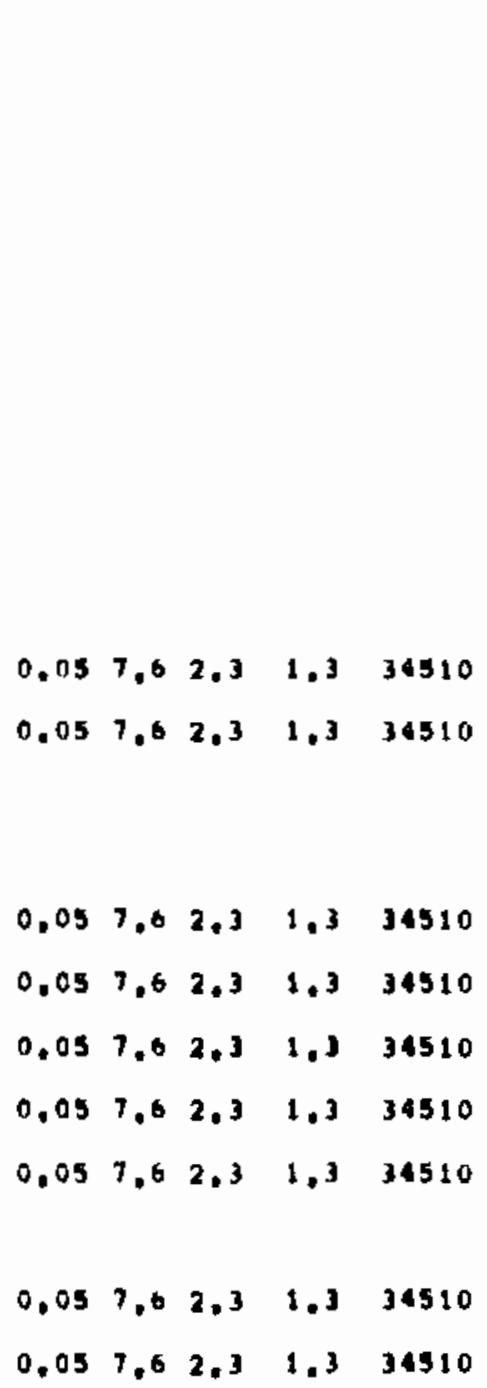

LIT 9

LI 9

LII 9

LII 9

LIT 9

LIT 9

L1T 9

LIT

LI

LIt 9

LII 2

LII 263

LIT 2

11 2

LII JA3

LIT 263

LIT 263

UIT 243

LII 263

LIT 263

L1I 263

$-7 \quad-59$ 
TAHLE II

MISCEILANEDUS COFRCSIUN INETHMATION

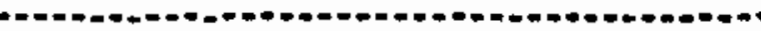

CheVice

STRESS PHANGE IN MOPERTY METHOD

ADATTTRNAL SAMPIE RESCATDTTRN

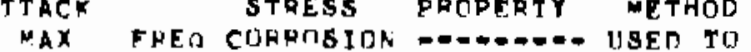

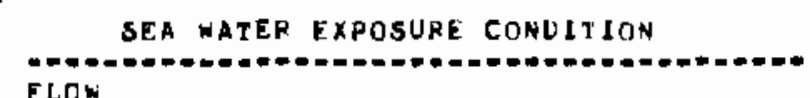

DEPTH PIT, CAT EL MEASURE

FLOW

TNCHFS SOCM KSI F/N IS PITS

FT' TEMP O2 SAL CU2 CU3 MARINE

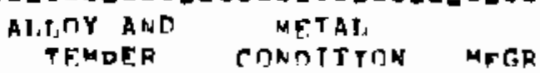

SEC PH (C) ML/L PPN PPM PPM GROWTH REFEHENCE

TDENT,
MUMAFF TrMoER cONOTTTOM

-.-.-

$15136051=76$

14 A 2 BกลITA

1493 KOG IOTA

1497 HOK1-Th

1908 6061-T6

$14996061-T 6$

$15 \cap 2$ HnK1-TG

1507 BnGI-TA

ISAB GAGI-TB

1517 4na107h

$151 R$ GDO1-Th

1520 GMA1-Th

1494 कก⿻1-76

1495 AOA1-Th

$15 \cap 0$ nnA $1=T A$

ISAT BOATETA

$15 \cap 3$ Bng 1 Th

jans nofinth

ISOB GOSTET

1509 anA $1=T$ T

(5, hOA $^{-T} \mathrm{~T}$

1314 AnA1-TA
$-100-100$

v

$.01 \mathrm{H}$

$.042 \quad .34$

n

INCIP DA

ond

$120 / 3$
$30 \quad 0 / 3$

$.5 \quad-42$

$-2 \quad-57$

$0 \quad-9$

$\begin{array}{ll}.055 & .11 \\ .051 & .20 \\ .065 & .43 \\ .060 & .25\end{array}$

$\begin{array}{ll}17 & 0 / 3 \\ 30 & 0 / 3 \\ 17 & 0 / 3 \\ 31) & 0 / 3\end{array}$

$0.057,62,3 \quad 1,334510$

$0.057 .6 \quad 2,3 \quad 1.3 \quad 34510$

LIT 263

LII 263

LIT 2 b 3

$0,107,5 \quad 5.0 \quad 0.4 \quad 34360$

$0.107 .55 .0 \quad 0.434360$

LII 263

LII 2 W 3

VAF $B, 1 \quad 16 \quad 3.2533510$

LIT 2 is

$0.107 .55,0 \quad 0.4 \quad 34360$

LIT 243

$0.107 .55 .0 \quad 0.434360$

LIT 2 W 3

$0.107 .55 .0 \quad 0.4 \quad 34360$

LIt 263

$0.107 .55 .0 \quad 0.434360$

LIT 20 J

VAP B.1 16 5.2533510

[1T 263

$0.057 .7 \quad 2.2 \quad 1.6 \quad 34400$

LIT 26 ]

LIT 263

0.107 .55 .00 .434360

LIT 2 +3

VAF $8,1 \quad 16 \quad 5,25 \quad 33510$

LIT 263

LIT 243

$0.057 .72 .2 \quad 1.634400$

LIT 263

$0.057 .72 .2 \quad 1.6 \quad 34400$

211243

$0.107 .55 .0 \quad 0.434360$

LII 263

$0.107 .55 .0 \quad 0.434360$

LII 263

$0.057 .72 .2 \quad 1.6 \quad 34400$

L: IT $2+3$ 
TABLE II

MISCELLANEOUS CORROSION INFURMATION

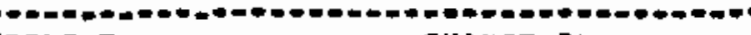

CREVICE

CREVICE

ATRESS PROFERTY METHOD

ADAITYMNAL SAMPLE DESCRTDTJON

TTACK

SEA WATER EXPOSURE CONDITION
FLOH

IOFT.

ALTY THO

METAT.

DEPTH PIT, COAOSION TS EL MEABURE

FLOW

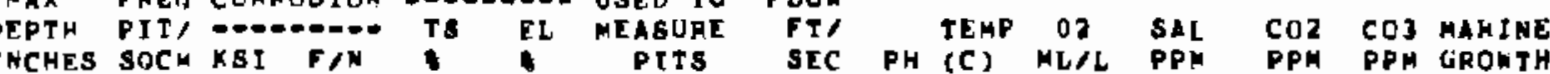
TEMPE CONDITION MFGR

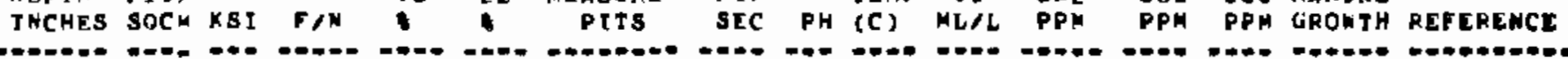

\begin{tabular}{|c|c|c|c|c|c|c|c|c|c|c|c|c|c|c|c|}
\hline $15 i 9$ & $9041-76$ & & & & & & & -15 & .70 & & 0.10 & 7,5 & 5.0 & 0.4 & 34360 \\
\hline 1504 & $6061-t x$ & & & INCIP & & & & & & & VAA & 0,1 & 16 & 5.25 & 33310 \\
\hline $14 A_{6}$ & $6061-T 6$ & & & .065 & .02 & & & & & & 0.05 & 7.6 & 2.3 & 1,3 & 34510 \\
\hline 1497 & $B_{6} t_{t}=T_{G}$ & & & PERT & & & & & & & 0.05 & 7.6 & 2.3 & 1.3 & 34510 \\
\hline 1498 & $6061-T 6$ & & & .063 & & & & & & & 0.05 & 1.6 & 2.3 & 1.3 & 34510 \\
\hline 1499 & 60K1-T6 & & & .048 & .22 & & & & & & 0.05 & 7.6 & 2.3 & 1,3 & 34510 \\
\hline 1490 & Gnal-Th & & & 0 & & & & & & & 0.05 & 7.6 & 2.3 & 1.3 & 34510 \\
\hline 1491 & $6061-76$ & & & 0 & & & & & & & 0,05 & 7.6 & 2,3 & 1.3 & 34510 \\
\hline 1492 & $60 \times 1=76$ & & & .075 & .06 & & & & & & & & & & \\
\hline 1493 & bAAt-TA & & & & .23 & & & & & & & & & & \\
\hline 1515 & GOA1-T6 & & . & & & & & -24 & .73 & & 0.05 & 7.6 & 2.3 & 1,3 & 34510 \\
\hline 1 คด & $6061-76$ & MTLt FIHISH & a LeOA & & & & & +1 & +5 & $x-S E C T$ & & & & & \\
\hline 1909 & $6061-7 n$ & MET $7072+P T$ & ALCOA & & & & & -27 & -24 & & & & & & \\
\hline 1810 & $6061-76$ & $A I D I N+P T$ & $A L C O A$ & & & & & +2 & +7 & & & & & & \\
\hline 1011 & $6051=76$ & MACHIMEN & A LCOA & & & 30 & $0 / 1$ & & & & & & & & \\
\hline 1494 & $6061-T h$ & & & .060 & .06 & & & & & & 0.05 & 7.5 & 2.6 & 1.2 & 34510 \\
\hline$\$ 493$ & Onat $=7 x$ & & & .066 & .05 & & & & & & 0.05 & 7.5 & 2.6 & 1.2 & 34510 \\
\hline 1496 & $6061-76$ & & & 0 & .09 & & & & & & & & & & \\
\hline 1516 & $6 n B 1-76$ & & & & & & & -11 & -61 & & 0.05 & 7.5 & 2.6 & 1.2 & 34510 \\
\hline 4576 & $6064=76$ & & $\left.A C^{\prime}\right) A$ & .0028 & & & & & & X-5ECT & & & & & \\
\hline 1527 & $6041=T 6$ & & LCOA & & & & & & & X $\mathrm{BECT}$ & & & & & \\
\hline $15>9$ & $6061-56$ & & $\triangle I C O A$ & & & & & +1 & & $x=\sec T$ & & & & & \\
\hline
\end{tabular}

LIT 263

LIT 263

LII 2

LIT 263

LIT 26]

LIT 2

[IT 263

LIT 263

LIT 2

LIT 2

LIT 263

ALCOA 62

ALCOA 62

ALCOA 62

ALCOA 62

LIt 2

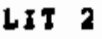

LIT 263

LIT 243

AbCOA 10

ALCOA 16

ALCOA 16 
TABLF I I

MISCFIALANEUIJS CURACSION THFORNATTOH CRFVICE CHANGE TN METHED STRESS PROPERTY METHCO
TTACK ADATTITNAL SAMPTE. DTERETHTTON

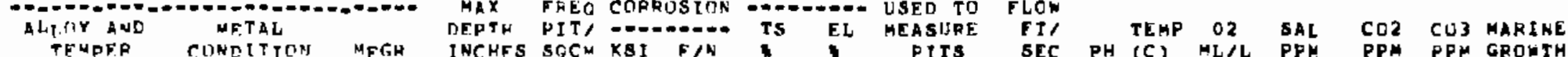

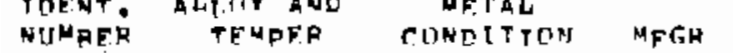
INCHFS SGCM KSI F/N PITS

SEC PH (C) ML/L PPM PPM PPM GROWTH RETERENCE Non

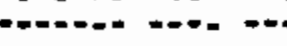

$+\infty+\cdots$

ALCOA 10
ALCOA 10
ALCOA 11
ALCUA 11
ALCOA 11
ALCOA 11
ALCOA 12
ALCOA 12
ALCOA 12
ALCOA 12
ALCUA 11
ALCOA 16
ALCOA
ALCOA
ALCUA 0
ALCOA 8
ALCOA
ALCOA
ALCOA 17

\begin{tabular}{|c|c|c|c|c|}
\hline & & .7 & -67 & $X=S F C T$ \\
\hline & & -1 & -12 & $x-\sec T$ \\
\hline & & -16 & -89 & $X-S E C T$ \\
\hline & & -7 & .76 & $x-\sec T$ \\
\hline & & -24 & -87 & $x=\sec t$ \\
\hline & & -1 & -52 & $x \rightarrow \sec r$ \\
\hline & & -66 & & $X-S E C T$ \\
\hline & & -16 & & $x \rightarrow 5 F C$ \\
\hline & & -74 & & $X-G E C T$ \\
\hline & & -38 & & $x=\sec t$ \\
\hline & & -32 & -99 & $x-850$ \\
\hline & & -9 & & \\
\hline & & -14 & -85 & \\
\hline 31 & $0 / 2$ & -6 & -75 & \\
\hline & & $-B$ & -75 & \\
\hline 30 & $0 / 2$ & $-B$ & -70 & \\
\hline & & -6 & -59 & \\
\hline & $11 / 1$ & & & \\
\hline & & & & $x=\operatorname{sect}$ \\
\hline & & & & $X-S E C T$ \\
\hline & & & & $x-5 F C T$ \\
\hline 3 & $0 / 1$ & & & \\
\hline
\end{tabular}

ALCOA 10 MTLL FINTSH BLCOA MITE FINTSH ALCTA MILI FIMISH BLCDA RATTEO ATCOA PAINTED DCIJA ATSOA aLena DATTES ALCOA PAINTFD ALCOA

NIU. FINISH BLCIIA atiros

MTLI. FINISH APT. HTLI FINTSH ART NIT, FINTSH AHT, MI TIT FINISH DRT, NTI,T FINTSM APT. WITI FINISH ART.

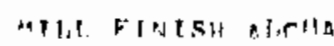
MIIF FIMISL MITHA

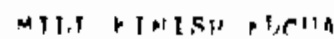
machintit alotia

\begin{tabular}{|c|c|c|c|c|}
\hline $191 \mathrm{~h}$ & $6 \cap A 1=T A$ & MTIL & F IN I SH & $A, C D$ \\
\hline $18+7$ & $6 \cap \times 1=T A$ & MILL & FINISH & $\triangle \mathrm{LCOA}$ \\
\hline 1 ค 12 & $B O A=T A$ & MTLI & FI I TSH & $\triangle 5 C 11 A$ \\
\hline 1 ค13 & $\operatorname{SnA} 1=T A$ & $M ! L I$ & $F[N I S H$ & a $1 \mathrm{C}$ \\
\hline 1014 & GOA $1=T G$ & PATN & TEn & ser \\
\hline 1 A 15 & $G 0 \dot{B} 1=T_{6}$ & PAINT & TE. D & Let \\
\hline 1530 & SOSt $=T_{n}$ & & & Ats \\
\hline 1531 & $A \cap A 1-T A$ & & & A Lens \\
\hline 1532 & AOA1-TA & DATAT & TEn & a Lec \\
\hline 1533 & SOA1-TA & PAINT & TFD & $A L C C$ \\
\hline TSA & $A \cap A_{B}=T_{A}$ & NIUI. & FInISH & A J \\
\hline 1525 & GnGt=Th & & & AliroA \\
\hline 1872 & $G_{A B}=T B$ & MTLL. & FINISH & $Q_{T}$. \\
\hline 1973 & $G \cap G 1=T G$ & : $T \mathbf{L}, \boldsymbol{T}$ & Fintsh & $A R T$. \\
\hline $\ln 5$ & $A \cap G I=T G$ & מוT & FI I I I SH & $\triangle \mathrm{NT}$ \\
\hline $\ln 6$ & $K \cap K 1-T h$ & MI I t & FINIS4 & $\triangle \mathrm{RT}$. \\
\hline 307 & $A \cap A 1-T A$ & $\omega \Gamma t, T$ & FINTSH & $p_{1}$. \\
\hline$T \cap A$ & $n \cap A B=T h$ & $=21.1$ & FINISH & AR. \\
\hline $7+9$ & $G A K I=T_{H}$ & $411, t$ & FINTSH & $A, \Gamma^{114}$ \\
\hline $73 n$ & hOGI-Th & NIIT & FIM IS: & DITIAa \\
\hline 737 & AOA $1=T A$ & $M T I . T$ & $r I+15 \%$ & pin \\
\hline 739 & $G \cap_{A} 1=$ & $4 \Delta C$ & & $a$ \\
\hline
\end{tabular}


TABLE It

MISCELLANEOUS CORROSION INFOAMATION CAEVICE CHANGE IN ATTACX BTRESS PROPERTY METHOD ADMTTIONAL SAMPLE DEBCRTOTION HZOYAND ATTACT DEPTH PIT/ IOENT. MLOY AND METAL
NUMBPR TEMPER CONDITTON MFGR INCHES SQCM KSI T/N

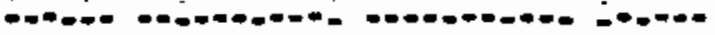

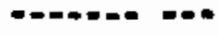

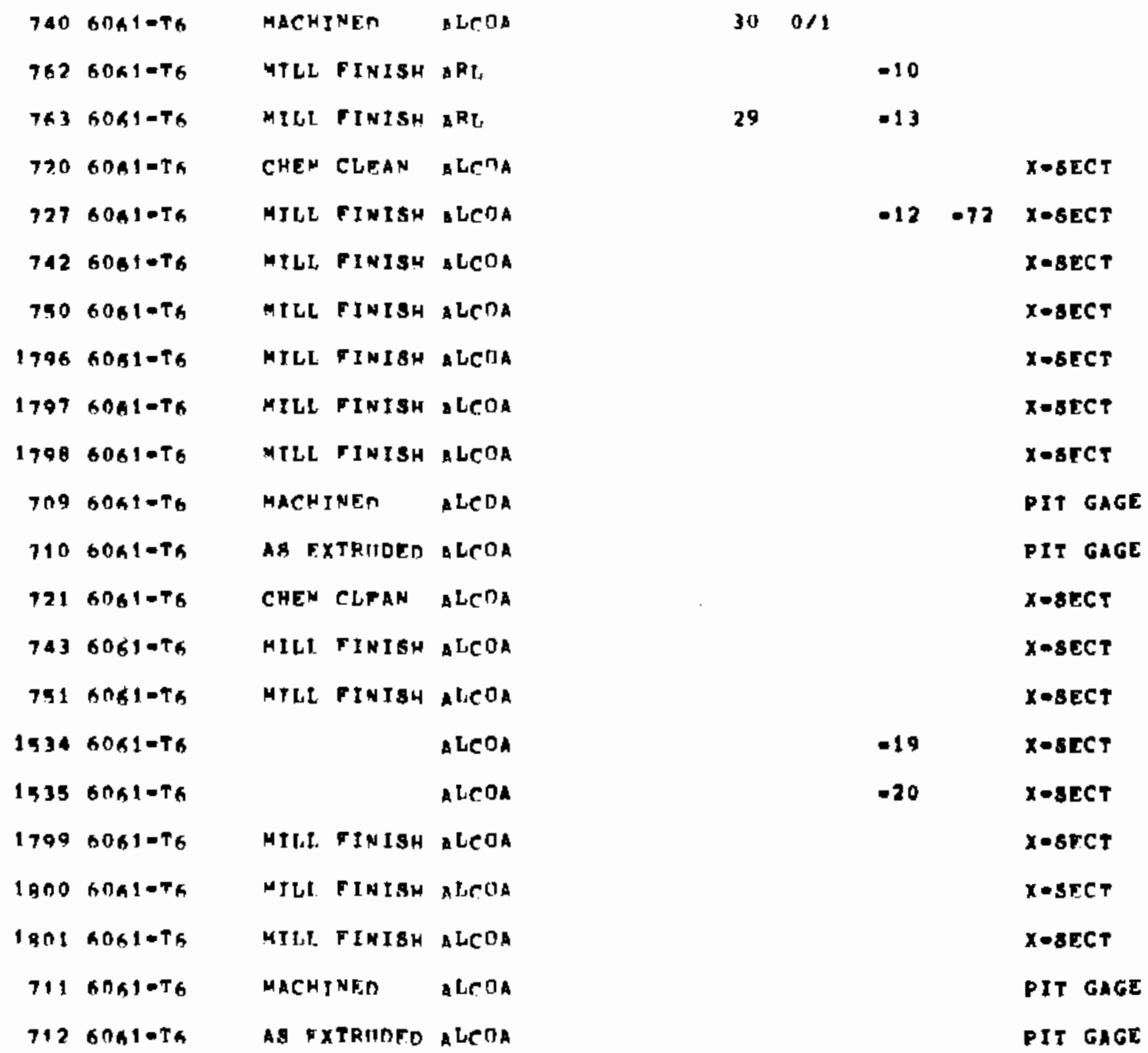

GARMAC ALCOA 17

ALCO 70

ALCOA 70

GARNAC ALCOA 17

ALCOA 41

ALCOA 69

ALCOA 69

ALCOA 68

ALCA 68

ALCA 48

ALCOA 71

ALCOA 71

BARMAC ALCOA IT

ALCOA 69

ALCOA 69

ALCOA 59

AlcoA 3 a

ALCOA 49

ALCUA 49

a LCOA 49

Alco 72

ALCOA 72 
TARLE II

MTSCELLANETUS CORRESION INF ORMATIUN

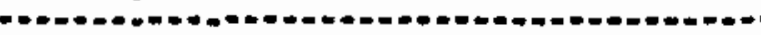
CREVICT

CREVICT CHAJGF IN
ADITTIONAL, SAMPLF, DFSCRTETTIN TTACK STRESS PROPERTY METHOD

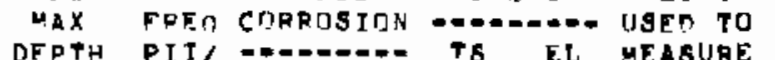
SEA MATER EXPCSURE CUNUITION

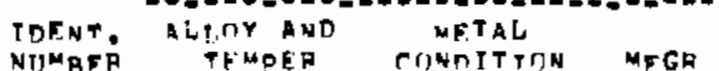
INCHES SCCM KSI F/N TS EL MEASUAE FLCi NMB MRE PM (C) HLA PPM PPM PPM GRONTH REEERLNCE

\begin{tabular}{|c|c|c|c|}
\hline 722 & GOA1-Th & CHEN CLFAN & ALCS \\
\hline 744 & $60 \mathrm{~A} 1 \cdot \mathrm{TA}$ & MTIT FINISH & $\triangle \operatorname{LCOA}$ \\
\hline 742 & AOAATA & MII,I FINISH & $\triangle L C O A$ \\
\hline $18 \cap 2$ & $50 A 1=T A$ & $M I I I F I N I S H$ & $A D C O A$ \\
\hline $\operatorname{lan} 3$ & AOAt-T6 & WILI FINISH & aLCOA \\
\hline 1 月04 & GOG:TA & MILL FINISH & $\triangle L_{C O A}$ \\
\hline 793 & GANI-TA & As FXTPInFn & $\Delta \cot A$ \\
\hline 714 & SnG $1=T h$ & AS FXTHIDFE & $\Delta \mathrm{I} C \mathrm{C} \mathrm{A}$ \\
\hline 723 & $6 \cap 51=7 G$ & CHEM CLFAN & $A L C \cap A$ \\
\hline 745 & ansi-ta & 4tLt FINISH & ALCOA \\
\hline $7 \times 3$ & $6 \cap 510+5$ & MTII FINISH & ALCOA \\
\hline 75 月 & SOKA.TA & A5 RILLED & $A L C O A$ \\
\hline 759 & angi-Ta & As RnLuFn & $A L C \cap A$ \\
\hline $\operatorname{lon} 5$ & moKl-Th & MILT FINISH & $A$ L.COA \\
\hline IRn & hnateta & YIUI. FI MISH & ${ }_{A} \operatorname{cen} a$ \\
\hline 1907 & $A \cap A C T A$ & YILE FINJSH & ALCria \\
\hline 715 & $G \cap A-T A$ & MACHINET & Aldrita \\
\hline 715 & $\sin 1=\pi k$ & as FXTHInEn & AleCta \\
\hline 724 & $A \cap A=T A$ & CHFE" CI, CAR: & Al.rila \\
\hline 745 & angiota & HII HJNTSU & $A t_{A} C_{A}$ \\
\hline 54 & $A \cap A_{A} 1=T_{A}$ & पाt.T & $A 1, C 0) A$ \\
\hline$\because 1$ & HSFl-Th & जACH & ATMUA \\
\hline
\end{tabular}

X-SECT
X-SECT
X-SECT
X-SECT
X-SECT
X-SFCT
PIT GAGE
PIT GACE
X-SFCT
X-SECT
X-SECT
X-SECT
X-SECT
X-SECT
X-SECT
X-SECT
PI GAGE
HIT GAGE
X-SFCT
-SECT
X-SFCT
HIT GAGE

BARMAC ALCUA 4

ALCOA 69

ALCOA 69

ALCOA 66

ALCOA 66

ALCOA 68

ALCOA 73

ALCUA 73

BARNAC ALCUA 17

ALCUA 69

ALCLA 69

BAFMAC ALCOA 52

BARNAC ALCOA 52

ALCUA 61

ALCUA 67

ALCOA 67

ALCOA 74

ALCOA 74

BAKINAC ALCUA 47

ALCU 69

ALCOA 69

ALCOA 53 
TABLE II

MISCELLANEOUS CORRESION INFORMATION CREVICE CREVICE STRESS PRDPERTY METHOD
ATPACE MAX FREO CORAOSION W-EDA-O USED TO

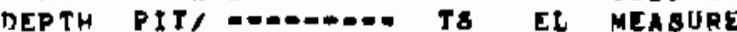

ADNTTIONAL BAMPLE DFSCRTDTION ALUY TND METAL

INCHES SACW KSI $\mathrm{YH}$

CASURE FT' TEMP 02 SAL COZ COJ MARIME

NUMBFA TEMPER CONDITION MFGR

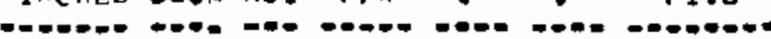

SEC PH (C) ML/L P M

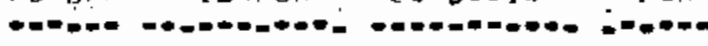

\begin{tabular}{|c|c|c|c|}
\hline 718 & $6061=76$ & As extkilOED & $\triangle \operatorname{LCDA}$ \\
\hline 725 & $6061=76$ & CHEM CLEAN & $\mathrm{AICOA}$ \\
\hline 747 & $60,1-76$ & MILL FINISH & ALCOA \\
\hline 795 & $6061-76$ & MTLL FINISH & $\triangle \angle C O A$ \\
\hline 748 & $6061 * T 6$ & MILI FINISH & nLCOA \\
\hline 736 & $6061-76$ & MTLI FINIBH & $4 \operatorname{LCOA}$ \\
\hline 749 & $6061=75$ & MTLL FINISH & a LCTA \\
\hline 737 & $6061-76$ & MILI FINISH & ${ }_{A} \operatorname{LCOA}$ \\
\hline 790 & $6091=76$ & & ALCOA \\
\hline 738 & AOA1-T6 & MTLI FINISH & ALCOA \\
\hline 741 & $60510+5$ & MACHTNE. & $\triangle L C O A$ \\
\hline 728 & $6061=76$ & MECHNED & ALCOA \\
\hline 729 & 6001-Th & MTLL FINISH & $\operatorname{AscOA}$ \\
\hline 731 & $6 \cap A 1=T 6$ & & ALCOA \\
\hline 1536 & $6051-T 911$ & & Alcna \\
\hline 1537 & $6061-7011$ & & Alena \\
\hline 1538 & $6061-7013$ & & $B$ LCOA \\
\hline 1539 & $6061-T a 13$ & & A LEDA \\
\hline 699 & $\sin 3=\pi 6$ & AS FXTAUDER & atictia \\
\hline h19 & SOA 3-Th & As TXTEIIDT: & ALCOA \\
\hline 614 & $6043-76$ & AS EXTHIIDED & a LCOA \\
\hline 615 & $60 \mathrm{~B} 3=\mathrm{TH}$ & As EXTRIMTn & ALCOA \\
\hline
\end{tabular}

PIT GAGE

PIT GAGE

$x=S E C T$

$x=\operatorname{seC} T$

$x=\operatorname{sect}$

$X=$ SECT

$X-\operatorname{sect}$

$\mathrm{X}-\boldsymbol{s e c} T$

$x=s \mathrm{ECT}$

$30 \quad 0 / 1$

$4.55 .18 \quad 35160$

$4,53.1035160$

$\begin{array}{lll}-13 & x-8 E C T \\ -15 & x+8 E C T \\ -13 & x-8 E C T \\ -12 & x-8 E C T \\ -1 & -20 \quad x-8 E C T \\ -3 & -2 \quad x-8 E C T \\ +7 & -2 \quad x-8 E C T \\ +6 & +7 & x-8 E C T\end{array}$

ALCOA 53

BARNAC ALCOA 4?

ALCU. 69

ALCOA 69

ALCOA 60

ALCOA 69

ALCOA 69

ALCOA 69

NONE LIT

NONE ALCOA 17

MONE ALCOA 17

NONE LIT 7

MOHE LIT

MONE LIT 8

ALCOA 59

ALCOA 50

ALCOA 59

Alcon 58

Alcon 10

ALCUA 10

ALCOA 11

ALCOA 11 
TALLF I

MTSCELANFUUS CIRACSITM TNFOAMATION

COFVTCE

\section{CREVTCE} SHAREST IN

PROPERTY METHON

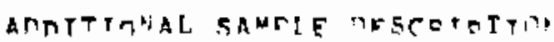
TACK

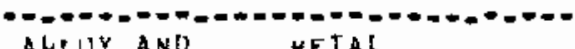

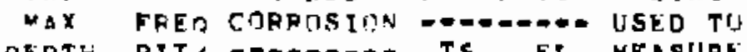

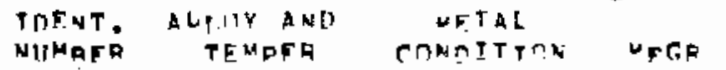
OFPTH PIT, JNRHES SOCA K8I FIM PITS

SEA WATEH EXPOSURE CONOITIUN FLQN TEMP O2 SAL CU2 CU3 MARIAE $\begin{array}{lllll}\text { FT' TEMP O2 } & \text { SAL CU2 CUB MARINE } \\ \text { SEC PH (C) MLIL PPU PPM PPM GROKTH REVERENCC }\end{array}$

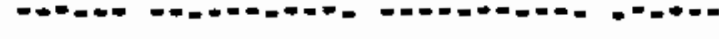

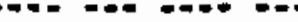

\begin{tabular}{|c|c|c|c|c|}
\hline his & 6CA 3-T & $\Delta !$ II & $2191 !$ & $\operatorname{sen} a$ \\
\hline 617 & OOK $1=T A$ & A) IJM & $215 \mathrm{ar}$ & $1207 \mathrm{~A}$ \\
\hline 610 & Gna B.TA & AS $\mathrm{F}$ & ח & ICts \\
\hline h'1 & 6חG 7-TA & 456 & XTRT+NE+ & t $t \in A$ \\
\hline 612 & $606^{3}+16$ & AIIIS & 21511 & $\operatorname{lcc} 2$ \\
\hline 613 & DกA3 $30 T A$ & At $: 1 \mathrm{~N}$ & $215 \mathrm{dr}$ & $\therefore \therefore+r^{11}$ \\
\hline 600 & GOKK-TA & $M T L L$ & FIA: IS:- & $: 2:$ \\
\hline $6 \cap 1$ & $6 \cap A K=T 4$ & $x+1$ & TINISL & $9=0$ \\
\hline 602 & $B D E K=T A$ & MIt. & $F I 4 I s:$ & $\geq 21$ \\
\hline $\sin 3$ & $6 \cap_{5} 6=74$ & NTL! & 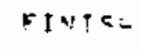 & 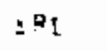 \\
\hline 504 & $A \cap A B=T G$ & MTt.T & JI: & $\therefore D$ \\
\hline $6 \cap 5$ & BOABOTA & MIL' & FINI<S & 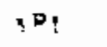 \\
\hline AOG & GOKK-Th & $4 \mathrm{TLL}$ & F't ISt & 2 的 \\
\hline ant & $A \cap G A=T A$ & Mrt & FTHIS:- & $\therefore T$ \\
\hline$A O R$ & GDAKETG & *TLL & FIVISL & $\because$ \\
\hline 609 & GOGA=TA & 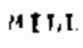 & $E \mathrm{~J} ; 1 \mathrm{SL}$ & Iآنز \\
\hline 395 & S $151=T A$ & 4 & $F I N_{I} I S H$ & $\operatorname{TSA}$ \\
\hline 507 & $B 19:=T 4$ & MTLLL & Fu & 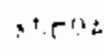 \\
\hline 504 & HIS10Th & 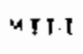 & $F_{T}+1<\ldots$ & $\cdot \cos$ \\
\hline 500 & $n+1-T h$ & "AJi:? & FI'IS:I & 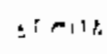 \\
\hline on & $B+4+-T 4$ & MTI.T & $F$ F & $\therefore 1$ \\
\hline 1949 & 510 & ती $\{1,1$ & FIALSL & $\therefore=4$ \\
\hline
\end{tabular}

\begin{tabular}{|c|c|c|c|c|}
\hline & & -2 & -58 & $x=5 \varepsilon C_{T}$ \\
\hline & & +6 & -20 & $x=5 E C T$ \\
\hline & & +4 & & $x=\operatorname{sect}$ \\
\hline & & +2 & & $x=S E C T$ \\
\hline & & -10 & & $x-\operatorname{sic} t$ \\
\hline & & -7 & & $X$-SECT \\
\hline & & -25 & -82 & \\
\hline & & $-4 R$ & -91 & \\
\hline & & .23 & -85 & $x-\operatorname{sect}$ \\
\hline & $0 / 2$ & & & \\
\hline & & -38 & -92 & \\
\hline 39 & $1 / 2$ & .71 & -00 & \\
\hline & & -49 & -92 & \\
\hline 39 & $2 / 2$ & & & \\
\hline & & -48 & -92 & $x=\sec \tau$ \\
\hline & $3 / 3$ & & & \\
\hline 19 & & -28 & & \\
\hline & & -13 & & \\
\hline 32 & & $-4 n$ & & \\
\hline & & -20 & & \\
\hline & & -11 & -03 & \\
\hline & & -6 & $-3 B$ & \\
\hline
\end{tabular}

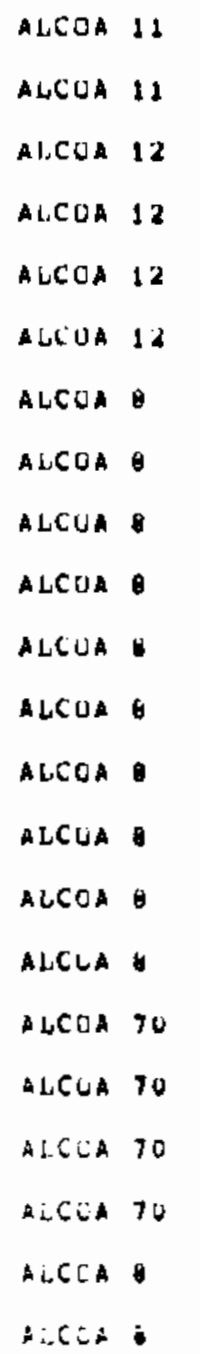


TAPLE. II

MISCELLANEOUS CORROBION INFORMATION

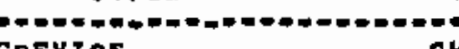
CPEYTCE

ATTACX BTRESE PROPERTY METHOD MAX TREO CORADSION -O..... USEO TO DEPTH PIT, INCHES SOCM KSI F/N

SEA HATER EXPOSURE CONDITION
FLOW
FI' TEMP OZ SAL CU2 COJ MAPINE
SEC PH (C) MLAL PPM PPM PPH GPOMTH HEFEREMCE

\begin{tabular}{|c|c|c|c|}
\hline 548 & $6351-74$ & MILL FINISH & A RI \\
\hline 1549 & $6351+74$ & MILI FINISH & $\mathbf{A} \mathbf{R}_{\mathbf{L}}$ \\
\hline 1550 & $63 \$ 1-76$ & MILC FINISH & $\mathbf{A R}_{\mathbf{T}}$ \\
\hline 1551 & $6351=76$ & MILL FINISH & $\Delta R_{t}$ \\
\hline 1552 & $6351-76$ & MILL FINIBH & $A R_{L}$ \\
\hline 1593 & $6351-76$ & MILL FINIBH & $\mathbf{A P}$ \\
\hline 1554 & $6351-76$ & MILL FINIBH & $A P_{L}$ \\
\hline 1555 & $6351-76$ & MILL FINISH & 81 \\
\hline 1556 & $6351-T 6$ & MACHINED & ALCOA \\
\hline 1557 & $6351=76$ & AS EXTATDED & ALCOA \\
\hline 1358 & $6391-T 6$ & MACH I NEN & ALCOA \\
\hline 1550 & $6351=76$ & As IXTRUDEn & aLCOA \\
\hline 1540 & $6351-T 6$ & MACHI MED & ALCOA \\
\hline 1561 & $6351-76$ & As FXTRIJDED & ALCOA \\
\hline 1562 & $6351=75$ & MACHINED & ALCOA \\
\hline 1563 & $6391 \bullet T 6$ & A\& EXTATIDED & atcon \\
\hline 1564 & $6341-T 6$ & MACHINEN & $\mathrm{LCOA}$ \\
\hline 1565 & $6391-76$ & AS EXTRIDER & $\triangle \mathrm{LCOA}$ \\
\hline 1566 & $6351-T_{h}$ & & \\
\hline 194 & ALC30030 & & $\triangle L C A N$ \\
\hline 192 & ALC $30033^{\circ}$ & & LCAN \\
\hline 193 & ALC $3003=$ & & LCAN \\
\hline
\end{tabular}

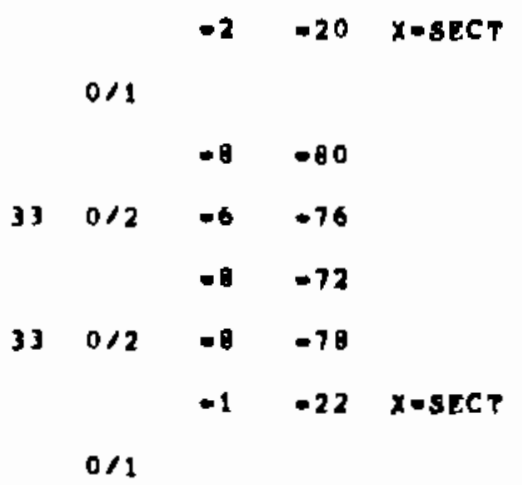

ALCOA
ALCOA
ALCOA
ALCOA O
ALCOA
ALCOA
ALCOA
ALCOA
ALCOA 71
ALCOA 71
ALCOA 72
ALCOA 72
ALCOA 73
ALCOA 73
ALCOA 74
ALCOA 74
ALCOA 53
ALCOA 53
LIT O
LIT 9
LIT 9
LIT 9


TAPLE II

MTSCELLANFIIIS CTIRRCSIOK TNFORMATIUN

PROPERTY WETHOO

ARATTIONAT SAMPLF DFSCDTRTTON

CRTITS

STRESS PANGE IN

MAX FREO CORRNSTUN *O"

AVTUV

DEPTH PIT, TS EL MEASURE

TDFNT:

TEMY
TEMPR

NETAL

INCHFS SOCM KSI FIN FITS

FT/ TEMP 02 SAL CO2 CU3 MARINE

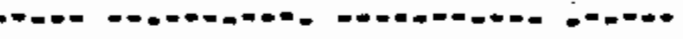

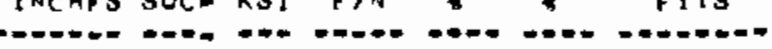

SEA WATEH EXFGSURE CONOITION

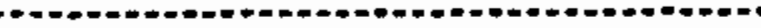
SEC PH (C) ML/L PEN PPM PPM GRCWTH PEFERENCE

\begin{tabular}{|c|c|c|c|c|c|c|c|c|c|c|c|}
\hline 1 ค 2 & $A l, r 3 O \cap 3=H_{4}$ & & & $\triangle \mathrm{LCOA}$ & +1 & & & & & & \\
\hline 129 & $\mathrm{ALC} 3 \cap \mathrm{O}_{3}-\mathrm{HI}_{4}$ & & & 0 & & & & .05 & 7.62 .3 & 1.3 & 34510 \\
\hline 160 & ATr $3003=\mathrm{H}_{4}$ & & & & 0 & +100 & & 0.05 & $7.6 \quad 2.3$ & 1,3 & 34510 \\
\hline 42 & $A L_{1} C_{3 O n}=H_{4}$ & & & $n$ & & & & 0.05 & 7.62 .3 & 2,3 & 34510 \\
\hline 193 & $\mathrm{ALC} 3003=\mathrm{H} \mathrm{L}_{4}$ & & & $A \operatorname{lich}$ & -5 & & $x-\operatorname{SEC} T$ & FLCw & & & \\
\hline 1 คด & $\mathrm{AT}_{1} \mathrm{C} 3003=414$ & MILE & FINISH & $\triangle \mathrm{JICOA}$ & -1 & -12 & $x=\operatorname{SFC} T$ & & & & \\
\hline 199 & $\mathrm{Al}_{1} \mathrm{C}_{3003}=\mathrm{H}_{4}$ & MIti & FINISH & $\sin C \operatorname{Con}$ & 0 & -17 & $x=\sec T$ & & & & \\
\hline 196 & $\sec 30 n 3=\mathrm{H}_{4}$ & $\operatorname{Mat} U$ & FINISH & $A I C C A A$ & +1 & +10 & $x=5 F C t$ & & & & \\
\hline 197 & $A I, C 30 O_{3}=\mathrm{H}_{4}$ & MTI.T. & FINIS4 & $\Delta$ Lena & +1 & -7 & $x-55 C T$ & & & & \\
\hline 194 & $A 1,53003=414$ & & & ALCOA & -2 & & $x=5 \pi c t$ & & & & \\
\hline 195 & ate $3(103-4)_{4}$ & & & $\triangle L C O A$ & -3 & & $x-\operatorname{SECT}$ & & & & \\
\hline 177 & AT.C3ONI-H:4 & & & ALCria & -1 & & & & & & \\
\hline 178 & $\mathrm{ALC} 3 \mathrm{On}_{3}=\mathrm{HI}$ & & & AT.CIIA & -1 & & & & & & \\
\hline 171 & AT.r 3 in $3-414$ & & & ALCTA & -3 & & & & & & \\
\hline 172 & $A L C 31103-414$ & & & a IJPA & -1 & & & & & & \\
\hline 179 & ATIR $3 \cap 03=H_{4}$ & & & ALCOA & -2 & & & & & & \\
\hline 190 & AT.ron $3=41$ & & & a teria & -1 & & & & & & \\
\hline 175 & AT. $+3003=412$ & & & a beos & 0 & & & & & & \\
\hline $1+5$ & ATr & & & atiCOA & .7 & & & & & & \\
\hline 201 & Atetonas & $v[t .1$ & $F[H] S H$ & $\triangle \mathrm{LC} \cap \mathrm{a}$ & +1 & -6 & $x$ SECt & & & & \\
\hline $2 \cap 2$ & $\operatorname{sic} 3010 a^{\circ}$ & 41,1 & H.1.5H & Aistis & +2 & 0 & $x=5 F C t$ & & & & \\
\hline 199 & Aldr300A* & $1 T 1,1$ & NIPISH & atcons & -1 & -6 & $x-\operatorname{sect} T$ & & & & \\
\hline
\end{tabular}

ALCOA 16

tit 3

LIT 263

LII 3

ALCOA 10

ALCOA 10

ALCOA 10

ALCOA II

ALCOA 11

ALCOA 12

ALCUA 12

ALCUA 16

ALCUA 16

ALCOA 16

ALCOA 16

ALCU io

ALCOA IO

alcua io

ALCUA 10

ALCOA 10

ALCOA IU

alcoA 11 
TABLE II

MISCELLANEOUS CORACSION INFORMATIUN

CREVICE

ATTACK STRESS PROPERTY METHOD MAPTH PIT, COROOSION FOA TS EL USEDTO

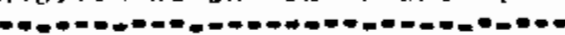
DEPTH PIT'
INCHFS SOCM KSI F/N TS EL MEASUPE

SEA WATER EXPOSURE CONDITIUN

TOU TIMP 02 SAL COZ COJ MARINE

SEC PH (C) ML/L PPH PPM PPM GROITH REFEHLNCE

NUMBTR TEMPER CNNDITION MFGR

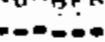

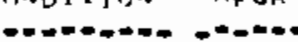

$00+0-0$
$-1-14 \quad x-5 E C T$

-3 $x$ NECT

$-3 \quad x \rightarrow 5 E C T$
ALCOA 11

ALCOA II

ALCOA 12

LII 9

LIT 9

LIT 9

LIT 9

LIT

LIT 9

LII 9

LII 9

LIT

A IJCAN

A LCAN 


\section{APPENDIX III \\ SERVICE HISTORIES}

Table No.

I

II

III

IV

$\mathrm{V}$

VI

VII

VIII

IX

\section{Caption}

5. S. Alcoa Clipper

OSW Test Bed Plant, Freeport, Texas Heat Reject Exchanger E-2I

Surface Condenser, Baltimore, MD

Surface Condenser, Freeport, TX

Surface Condenser, Texas City, TX

SS Alcoa SeaProbe

Lube Oil Cooler, Port Arthur, TX

Ammonia Condenser, Lake Charles, LA

Baltic Sea Water at Studsvik

AB Atomenergi Research station 

TABLE I

S. S. ALCOA CLIPPER

SHIPBOARD HEAT EXCHANGER - 3/4" OD $x .064 "$ WALL ALCLAD (INSIDE 7072) 3003-HI 4

Original

Cladding

Thickness(1)

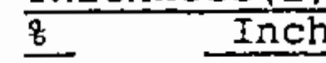

$16 \quad .0104$

$16 \quad .0104$

$16 \quad .0104$

$16 \quad .0104$

$16 \quad .0104$

16.0104

16.0104

$8 \quad .0052$

$16 \quad .0104$

$16 \quad .0104$

\begin{tabular}{|c|c|c|c|}
\hline Tubes & Years & $\begin{array}{c}\text { Cladding } \\
\text { Consumed } \\
\frac{8}{6}\end{array}$ & $\begin{array}{l}\text { Maximum } \\
\text { Remaining } \\
\text { Cladding } \\
\text { Thickness } \\
\text { Inch }\end{array}$ \\
\hline Seawater & $I$ & - & .0100 \\
\hline Seawater & 1 & 5 & ---- \\
\hline Seawater & 2.7 & 10 & ---- \\
\hline Seawater & 4 & - & .0100 \\
\hline Seawater & 4 & - & .0070 \\
\hline Seawater & 5 & 50 & .0051 \\
\hline Seawater & 7 & 53 & .0107 \\
\hline Seawater & 8 & 90 & .0050 \\
\hline Seawater & 9 & 50 & .0103 \\
\hline Seawater & 10 & 76 & .0102 \\
\hline
\end{tabular}

Maximum

Penetration of Core - Inch

Nondetected

Nondetected

Nondetected

Nondetected

Nondetected

Nondetected

Nondetected

.022

Nondetected

Nondetected

Note: (1) Nominal thickness: 8 is of tube wall thickness. 
Table II

OSW - TEST BED PLANT, FREEPORT, TEXAS

HEAT REJECT EXCHANGER E-21

RAW SEA WATER AT $110^{\circ} \mathrm{F}$ IN TUBES

\begin{tabular}{lcccc} 
& $\begin{array}{c}\text { Max. Pit } \\
\text { Depth } \\
\text { Inch }\end{array}$ & $\begin{array}{c}\text { Avg. Pit } \\
\text { Depth } \\
\text { Inch }\end{array}$ & Pits/In & \\
\cline { 2 - 5 } Original Tubes & After 6 Months in Test & & \\
\hline 3003 & 0 & 0 & 0 & Excellent condition \\
5052 & 0 & 0 & 0 & Excellent condition \\
6063 & 0 & 0 & 0 & Excellent condition \\
5050 & 0 & 0 & 0 & Excellent condition
\end{tabular}

Replacement Tubes After 6 Months in Test

\begin{tabular}{llll}
\hline 3003 & 0 & 0 & 0 \\
5052 & 0.049 & 0.030 & 0.01 \\
6063 & 0.049 & 0.030 & 0.01 \\
5050 & 0.049 & 0.030 & 0.01
\end{tabular}

Excellent condition

Pits and perforation under barnacle shells Pits and perforation under barnacle shells Pits and perforation under barnacle shells

Original Tubes That Remained in Test for 12 Months

\begin{tabular}{llll}
\hline 3003 & 0 & 0 & 0 \\
5052 & 0.020 & -- & -- \\
6063 & 0 & 0 & 0 \\
5050 & 0.049 & 0.020 & 0.01
\end{tabular}

Excellent condition

One deep pit

Excellent condition

Pits and perforation under barnacle shells

Source: Desalination Materials Manual prepared by Dow Chemical Company for OsW, 1975 May.

on page 4-108 it is reported pitting developed during the final six months of testing. 
TAPLF I T

MISCFLLANEUUS CORRCSIUN TNFCRMATJON

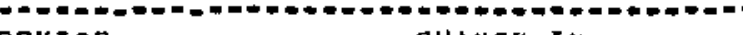

CREVICF STRESS PRANGE IN

TTACK STRESS PROPEPTY WFTHOD

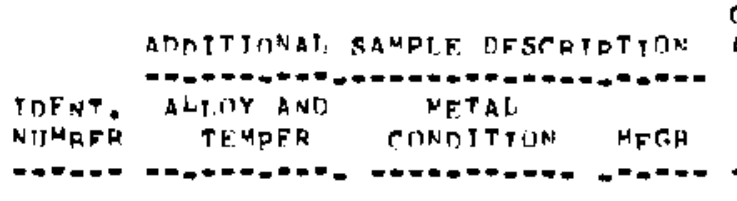

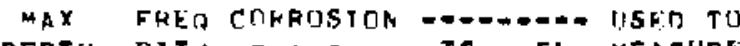

OFPTH PIT, TEMP O2 SAL CO2 COJ MARINE
INCHFS SOCM KSI FIN

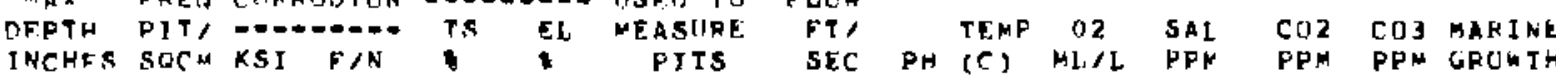

SEA WATER EXPOSURE CONDITION

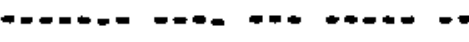

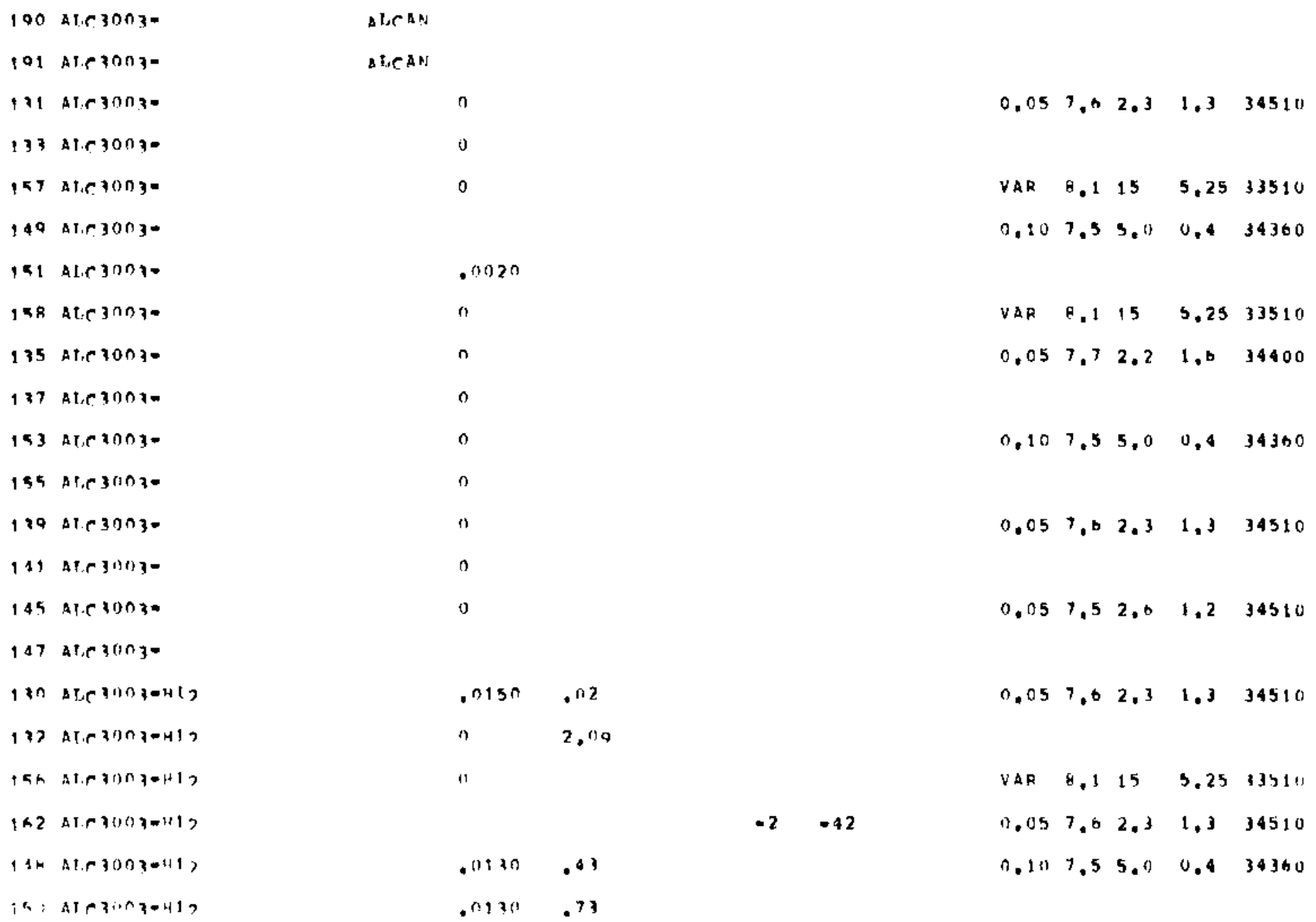

LIT 9

LIT 9

LII 243

LII 263

LII 243

LIT $2+3$

LIT 243

LII 243

LII 2 เ 3

LIt 263

LII 263

LIT 2G3

LII 2 t 3

LIT 263

LIJ 263

LIT 263

LIT 263

LII 263

I. 17243

$1,15 \quad 203$

LI1 263

1.11 2+3 
TABLE II

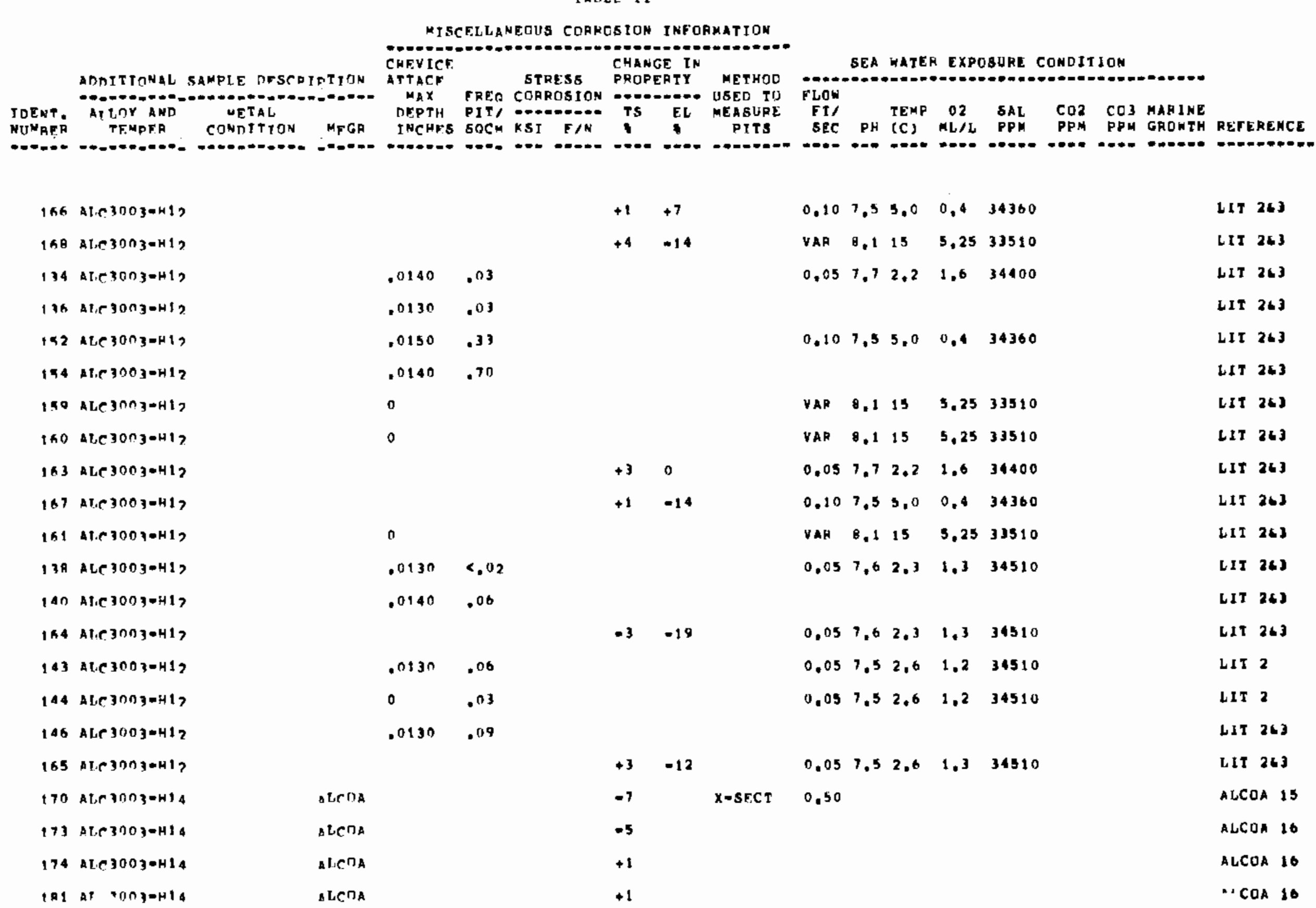


TABLE III

BALTIMORE 30, MARYLAND

CONDENSER WITH 3/4" OD $x .065 "$ WALL ALCLAD (INSIDE 7072) 3003-H14 TUBES

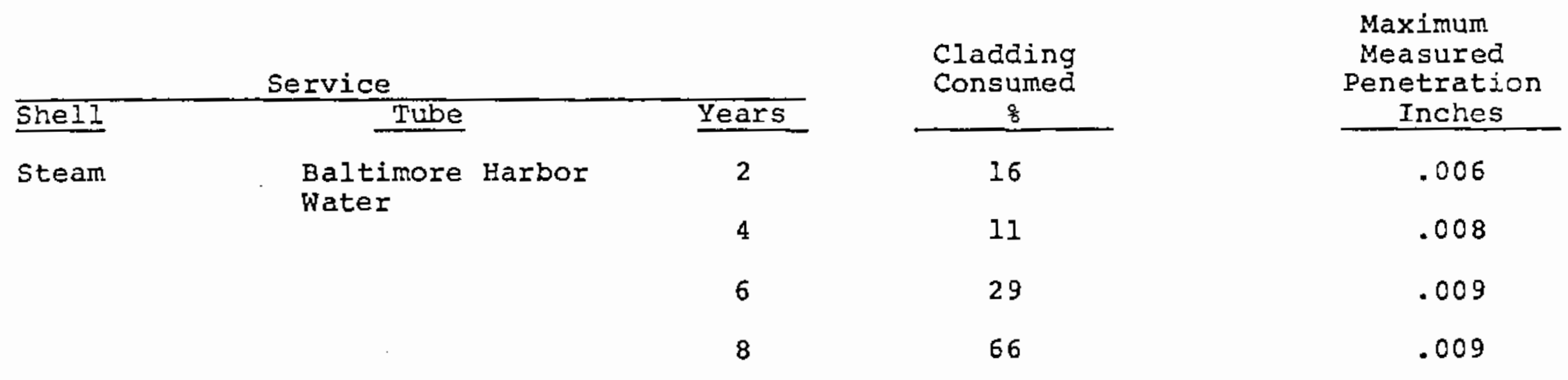


TABLE IV

FREEPORT TEXAS, CONDENSER, ALCLAD (INSIDE 7072) 3003 AND NONCLAD 3003 TUBES $3 / 4 "$ OD $x .065^{\prime \prime}$ WALI

\begin{tabular}{lcc} 
& Sexvice & \\
\cline { 2 - 3 } Shell & Tears \\
\cline { 2 - 2 } Stear & Seawater & 2 \\
Steam & Seawater & 2
\end{tabular}

Tube

Alloy

Alc. 3003

3003
Maximum

Measured

Penetration Inch

Cladding Not Perforated

.0038 
TABLE V

TEXAS CITY, TEXAS

CONDENSER - ALCLAD (INSIDE 7072) 3003 AND NONCLAD 3003 TUBES $3 / 4^{\prime \prime}$ OD $x .065^{\prime \prime}$ WALL

\begin{tabular}{|c|c|c|}
\hline & Service & \\
\hline She11 & Tubes & Years \\
\hline steam & Seawater & $11 / 4$ \\
\hline
\end{tabular}

$21 / 2$
Tube

Alloy

Alc. 3003

3003

Alc. 3003

3003
Remarks

scattered pits limited to cladding

scattered small pits

Broad shallow pits cladding protecting

scattered small pits deeper than at $11 / 4$ years 
ALUMINUM DESALINATION UNIT ABOARD THE ALCOA SEA PROBE CONDENSER AND EVAPORATOR TUBED WITH 5/8" OD $x .065 "$ WALL ALCLAD (BOTH SIDES 7072) 3003-H14 TUBES

\section{Condenser}

Shell 5454-H34 aluminum

Tubes 5/8" OD $x .065 "$ wall alclad (both sides 7072) 3003-H14

Water box - steel coated internally with Debecate

Service

Raw sea water in tubes (small barnacles formed)

Condensate in shell

7 years - No problems due to corrosion

\section{Evaporator}

Shell 5454-H34 aluminum

Tubes 5/8" OD $x .065^{\prime \prime}$ wall alclad (both sides 7072) 3003-H14

Tube sheet - Alclad (both sides 7072) 6061-T6, I" thick

water box - steel coated internally. with Debecate

Service

Engine jacket water in tubes

Hot sea water evaporating in shell

7 years - no corrosion problem 
TABLE VII

\section{LUBE OIL COOLER - PORT ARTHUR, TEXAS}

Tubes - Alclad (Inside 7072) 3003-H14

Coolant - Brackish water in Tubes

Service - After 5-6 years about 508 of cladding consumed, but no penetration of the 3003 core. The cooler remained in service. 


\section{TABLE VIII}

\section{AMMONIA CONDENSER - LAKE CHARLES, LA}

Tubes - Alclad (Inside 7072) 3003-H14

Coolant - Brackish water in tubes

Service - No examination or trouble reported in 4 years. Condenser remained in service. 
TABLE IX

ALCLAD ALUMINUM TUBES IN BALTIC SEA WATER AT STUDSVIK, AB ATOMENERGI RESEARCH STATION

\begin{tabular}{ll}
\multicolumn{2}{c}{ Composition of } \\
Baltic Sea Water at Studsvik \\
\hline Chloride & $3970 \mathrm{mg} / 1$ \\
Sulfate & $550 \mathrm{mg} / 1$ \\
Calcium & $72 \mathrm{mg} / 1$ \\
Sodium & $2450 \mathrm{mg} / 1$ \\
Magnesium & $79 \mathrm{mg} / 1$ \\
Oxygen & $8-14 \mathrm{mg} / 1$ \\
Hydrogen sulfide & $<0.1 \mathrm{mg} / 1$ \\
Ammonia & Not Detected \\
pH & 7.8
\end{tabular}

Composition of

\section{Tube Alloys}

SIS 4212, Al Si $1 \mathrm{Mg} \mathrm{(6351)}$

Clad inside with Al-zn 1 (7072)

SIS 4212, Al Si $1 \mathrm{Mg}$ (6351)

Clad inside with Al 99.99

Time 10,000 hrs. (13.7 mos.)

Velocity $2.5 \mathrm{~m} / \mathrm{sec}(8.2 \mathrm{ft} / \mathrm{sec}$.

\section{Remarks}

108 cladding removed by erosion - corrosion. Penetration no deeper than 140 er $\left(.0055^{\prime \prime}\right)$ 50-60\% cladding removed by erosion-corrosion. Penetration no deeper than 200_m $(.0079 ")$
Temperature $50^{\circ} \mathrm{C}\left(122^{\circ} \mathrm{F}\right)$
Concluded that at $50^{\circ} \mathrm{C}\left(122^{\circ} \mathrm{F}\right)$ velocity of $2.5 \mathrm{~m} / \mathrm{sec}(8.2 \mathrm{ft} / \mathrm{sec})$ too high for aluminum tubes tested. Further stated that heat exchangers containing aluminum tubes clad inside with Al $\mathrm{Zn} 1$ (7072) have operated problem-free in "Studsvik-water" at a maximum temperature of $20^{\circ} \mathrm{C}\left(68^{\circ} \mathrm{F}\right)$ and water velocities of less than $2.5 \mathrm{~m} / \mathrm{sec}(8.2 \mathrm{ft} / \mathrm{sec}$ ) for more than 15 years.

Ref; "Corrosion Tests in Baltic Seawater on Heat Exchanger Tubes of Various Metallic Materials", Henrikson and Knutsson. 
Final Report on Special Agreement No. B-31942-A-E

ivo. of

Copies

1 Mr. A. A. Churm

DOE Chicago Patent Group

9800 South Cass Avenue

Argonne, IL 60439

27 DOE Technical Information Center P. O. Box 62

Oak Ridge, TN 37830

1 Dr. Robert A. Bonewitz

Section Head

Aluminum Company of America

Alcoa Technical Center

Alcoa Center, PA 15069

1 Dr. H, Lee Craig, Jr.

Associate Professor

Division of Ocean Engineering

School of Marine \& Atmospheric Science

University of Miami

10 Rickenbacker Causeway

Miami, FL 33149

1 Mr. John De Palma

Code 3432

Room C-316, BIdg. 1105

U.S. Naval Oceanographic Office

Bay St. Louis, MS 39522

1 Dr. Stephen Dexter

College of Marine Sciences

Complex

University of Delaware

Lewes, DE 19958

1 Dr. J. G. Fetkovich

Department of Physics

Carnegie Mellon University

Schenley Park

Pittsburgh, PA 15213

1 Dr. Ralph Mitchell

Harvard University

Pierce Hall

Cambridge, Mass. 02138

1 Mr. Fred C. Munchmeyer

Dept. of Mechanical Engineering

University of Hawaii

2540 Dole Street

Honolulu, HI 96822
No. of

Copies

2 Mr. G. L. Liffick

DOE Richland Operations

Programs Division

Richland, WA 99352

5 Technical Information Files 3760 Building

Pacific Northwest Laboratory Richland, WA 99352

$1 \mathrm{Mr}$. Joe Rynewicz

Ocean Systems

Research \& Develop. Div.

Lockheed Missils \& Space Co.

ORGN. 57-20, Bldg. 150

1111 Lockheed Way

Sunnyvale, CA 94088

1 Dr. Elric W. Saaski

Sigma Research, Inc. 2952 GWW

Richland, WA 99352

1 Mr. William Sheppard

NOAA Data Buoy office

National Space Technology Iab

Bay St. Louis, MS 39529

1 Dr. Harold Vind

Research Chemist

Materials Science Division

Civil Engineering Laboratory

Naval Construction Battalion Center

Port Hueneme, CA 93043

1 Prof. Kenneth J. Bell

School of Chemical Engineer.

oklahome state University

Stillwater, OK 74074

1 Prof. James G. Knudsen

Director of Energy Research

\& Tech. Engineering

Experimental Station

Covell Hall 219

Oregon State University

Corvallis, OR 97331

1 Dr. Richard N. Lyon

Energy Division

Oak Ridge National Laboratory

P.O. Box X

Oak Ridge, TN 37830 
1 Mr. Jack M. Nilles

Southern Calif., Univ, of

University Park

Los Angeles, CA 90007

1 Mr. Robert E. Lacey

Southern Research Institute

Birmingham, AL 35205

1 Mr. Robert H. Douglass

TRW Inc., DSSG

One Space Park

Building 81, Room 1538

Redondo Beach, CA 90278

1 Mr. Frank Notaro

Union Carbide Corporation

Linde Division

Oak Ridge National Laboratories

P. O. Box 4

Oak Ridge, TN 37830

1 Ms. Brenda Little

NORDA - NSPL

Building 1105

Bay st. Louis, MS 39520

1 Mx. David Price

U.S. National Oceanic \& Atmospheric Administration

6010 Executive Blvd.

Rockville, MD 20852

1 Mr. Michael McCormick

U.S. Naval Academy

Annapolis, MD 21402

1 Mr. Steve A. Piacsek

U.S. Naval Research Laboratory

4555 Overlook Avenue

washington, D.C. 20375

1 Mr. Thomas E. Little

Westinghouse Electric Corp.

Oceanic Division

P. O. Box 1488

Annapolis, MD 21404

1 Mr. James W. Mavor, Jr.

Woods Hole Oceanographic Inst.

Woods Hole, MA 02543

1 Mr. Eugene J. Barnseff

Westinghouse Electric Corp.

P. O. Box 9175, N-206

Philadelphia, PA
1 Dr. Clarence Zener

Carnegie-Mellon University

Pittsburgh, PA 51213

1 Dr. Stuart Ridgeway

$R$ \& D Associates

P. O. Box 9695

Marina del Rey, CA 90291

1 Mr. Dale Sands

Interstate Electronics Corp.

707 E. Vermont Road

Anaheim, CA 92850

1 Mr. D. T. Hamilton, Jr.

AES/DBER

Department of Energy

Washington, DC 20545

1 Mr. Eric Adams

Massachusetts Inst. of Tech.

MIT 48-315

Cambridge, MA 02139

1 Mr. E. Franklin Johsnon

National Oceanographic Data

Center

2001 Wisconsin Ave., N.W.

Washington, DC 20235

$1 \quad$ Mr. Jantes Sandusky

Energy \& Environment Division

Lawrence Berkeley Lab

University of California

Berkeley, CA 94720

1 Mr. John B. Thomasian

Energy \& Environmental Analysis

1701 No. Fort Myer Drive

Suite 1213

Arlington, VA 22209

1 Ms. Pat Wilde

University of California

438 Hearst Mining Building

Berkeley, CA 93940

1 Mr. Peter Duncan

Tetra Tech., Inc.

1911 No. Fort Myer Drive

Arlington, VA 22209 
1 Mr. W. R. Suratt DSS Engineers, Inc. 7483 Northwest 4 th St.

Fort Lauderdale, FL 33317

$1 \mathrm{Mr}$. Ortwin Von Zweck

Dept. of Oceanography \& Ocean Engineering Florida Inst. of Tech. Melbourne, FL 32901

$1 \mathrm{Mr}$. W. R. McCluney Florida Solar Energy Center 300 State Road 401 Cape Canaveral, FL 32920

1 Mr. Edward J. Tachupp General Electric Company/TEMP 777 Fourteenth St., N.W. washington, D.C. 20005

1 Mr. Cullen M. Sabin Geoscience, Ltd. $410 \mathrm{~S}$. Cedros Ave. Solana Beach, CA 92075

$1 \mathrm{Mr}$. P. H. Hadley, Jr. Gibbs \& Cox, Inc. 40 Rector Street New York, NY 10006

1 Mr. Roderick A. Barr Hydronautics, Inc.

7210 Pindell School Road Laurel, MD 20810

1 Mr. Derek P. Gregory Energy Analysis Institute of Gas Technology $3424 \mathrm{~S}$. State Street ITT Center Chicago, IL 60616

1 Dr. Malcom D. Fraser Intertechnology Corp. 1001 Main street Warrenton, VA 22186

1 Mr. Robert Makofski Applied Physics Laboratory John Hopkins University Laure1, MD 20810

$1 \mathrm{Mr}$. John Nicol

Physical Systems Section Little, Arthur D., Inc. 28 Acorn Park Cambridge, MA
1 Mr. Lloyd Trimble Lockheed Missiles \& Space Co. P. O. Box 504 Sunnyvale, CA 94088

1 Dr. Jon McGowan

Mechanical Eng. Department Massachusetts, Univ. of Amherst, MA 01002

1 Mr. John J. Ditmars Argonne National Lab. Energy \& Environmental Div. 9700 South Cass Ave. Argonne, IL 60439

1 Mr. Paul Wolff Ocean Data Systems, Inc. 6000 Executive Blvd. Rockville, MD 20852

1 Mr. John H. Nath Dept. of Mechanical Engineering Oregon State University Corvallis, OR 97331

1 Mr. David L. Thomas Icthylogical Associates, Inc. Box 700 R.R. 2, West Brook Lane Absecon, NJ 08201

1 Mr. Fred Vukovich

Research Triangle Institute Research Triangle Park, NC 27709

1 Mr. N. Basar

Rosenblatt, M., \& Son, Inc. 350 Broadway

New York, NY 10013

1 Mr. Duane T. Hove

Science Applications, Inc.

One Continental Plaxa, Suite 310

101 Continental Boulevard

El Segundo, CA 90245

1 Mr. J. Hilbert Anderson

Sea Solar Power, Inc.

1615 Hillock Lane

York, PA 17403

1 Mr. A. J. Haskel

Society of Naval Architects \& Marine Engineering (SNAME)

Suite 1369, One World Trade Ctr. New York, NY 10048 
No. of

Copies

1 Mr. Sigmund sronich

DOE - Div. of Solar Technology

600 E. Street N.W.

Washington, DC 20545

1 Mr. William Smith

DOE - Div. of Solar Technology

600 E. Street N.W.

Washington, DC 20545

1 Dr. Lloyd Lewis

DOE - Div. of Solar Technology

600 E. Street N.W.

washington, DC 20545

1 Mr. Wilbur Sherwood

DOE - Div. of Solar Technology

600 E. Street N.W.

washington, D.C. 20545

1 Mr. Norman Sather

Argonne National Laboratory

9700 South Cass Ave.

Argonne, IL 60439

1 Mr. Charles Bretschneider

Bretschneider Consultants

2600 Pualani Way

Honolulu, HI 96815

1 Mr. H. H. Sephton

College of Engineering

office of Research Services

Univ. of Calif. at Berkeley

Berkeley, CA 94720

1 Mr. Gerald Wick

Institute of Marine Resources

Univ. of Calif. at San Diego

La Jolla, IA 92093

1 Mr. Abrahim Lavi

DOE-Div. of Solar Technology

600 E. Street, NW

Washington, DC 20545

1 Mr. Frank Mathews

Colorado School of Mines

Golden, Co 80401

$1 \mathrm{Mr}$. Robert Rothfus

Dept. of Chemical Engineering

Carnegie-Mellon University

Schenley Park

Pittsburgh, PA

15213
No. of

Copies

1 Mr. Dennis Brenning

Lockheed Center for Marine

Research

6350 Yarrow Drive

Carlsbad, CA 92008

1 Mr. Walter K. Boyd, Manager

Corrosion \& Electrochemical

Technology section

Chemistry Department

Battelle Columbus Laboratories

505 King Avenue

Columbus, $\mathrm{OH} \quad 43201$

1 Dr. William A. Corpe

Prof. of Biological Sciences

Dept. of Biological Sciences

Columbia University

New York, NY 10027

1 Dr. Richard Drisko - Navy

Senior Projects Scientist

Materials Science Division

Civil Engineering Laboratory, NCBC

Port Hueneme, CA 93043

1 Dr. Charles Gibson

Marine Biology Program Leader

Marine Research

Route 5, Box 1000

Sequim, WA 98382

1 Dr. E. C. Haderlie

Prof. of Oceanography

Naval Postgraduate School

Monterey, CA 93940

1 Dr. Francis L. La Que

Claridge House, Apt. \#803

Claridge Drive

Verona, NJ 07044

$1 \mathrm{Mr}$. Lyle D. Perrigo, Manager

Biofouling Project

Battelle-Northwest

P. O. Box 999

Richland, WA

99352

1 Dr. Eugene, Silva, Asst. Direct.

Ocean Facilities Program Office

Naval Facilities Eng. Control 200 Stovall Street

Hoffman Building \#2

Alexandria, VA 22332 
No. of

Copies

1 Mr. Eugene H. Kinelski

DOE - Div. of Solar Technology

600 E. Street NW

Washington, DC 20545

1 Mr. V. J. Castelli

Ocean Environment \& Fouling Branch

Code 2853, Materials Department

Naval Ship \& Development Center

Annapolis Laboratory

Annapolis, MD 21402

23 OTEC Biofouling \& Corrosion Project

Battelle Pacific Northwest Laboratory

Richland, WA 99352

1 Mr. John W. Michel, Manager

OTEC Heat Exchange Project Activities

Union Carbide Nuclear Division

Oak Ridge National Laboratories

BoX Y

Oak Ridge, TN 37830

1 Dr. Rober L. Molinari

NOAA/AOML

15 Rickenbacker Causeway

Miami, FL 33149 
